\title{
Near-explicit multiphase modeling of halogen chemistry in a mixed urban and maritime coastal area
}

Erik H. Hoffmann ${ }^{1}$, Andreas Tilgner ${ }^{1}$, Ulrike Vogelsberg ${ }^{2}$, Ralf Wolke ${ }^{2}$, and Hartmut Herrmann ${ }^{1, *}$

[1] Atmospheric Chemistry Department (ACD), Leibniz Institute for Tropospheric Research (TROPOS), Leipzig, Germany

[2] Modeling of Atmospheric Processes Department (MAPD), Leibniz Institute for

Tropospheric Research (TROPOS), Leipzig, Germany

\section{Supporting Information}

Number of pages: $\quad 84$

Number of figures: 6

Number of tables: $\quad 8$

* Corresponding author: Hartmut Herrmann, Permoserstr. 15, 04318 Leipzig, Germany Phone: +49 341 2717-7024, E-mail: herrmann@tropos.de 


\section{Figures}

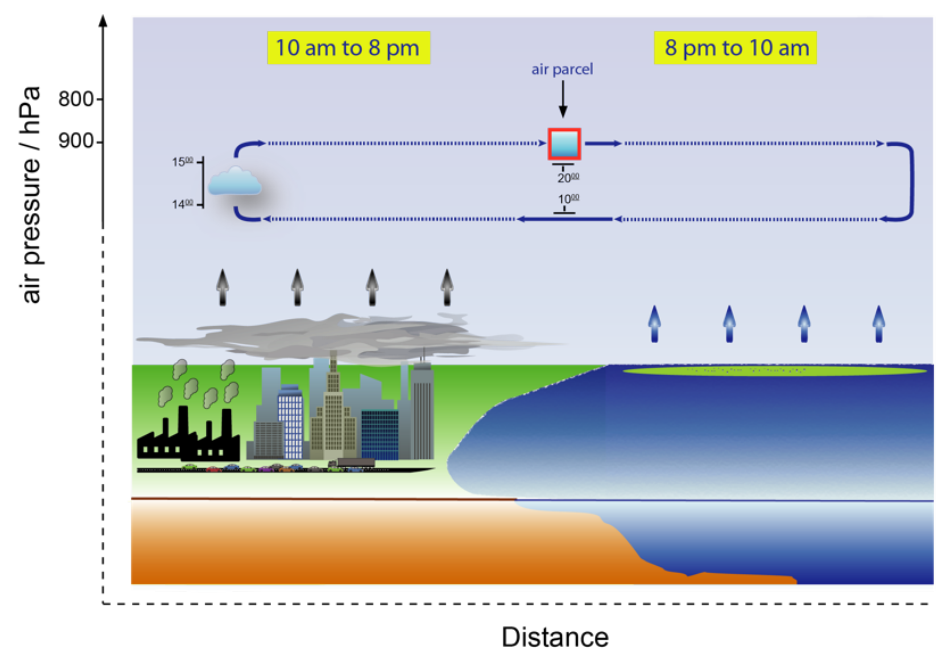

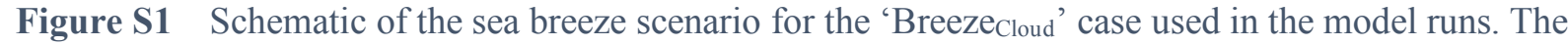
grey arros represent anthropogenic influenced coastal emissions of trace gases, whereas the blue arrows represent marine coastal emissions interacting with the air parcel. The time the air parcel spends over the continental and marine environment is also furnished.

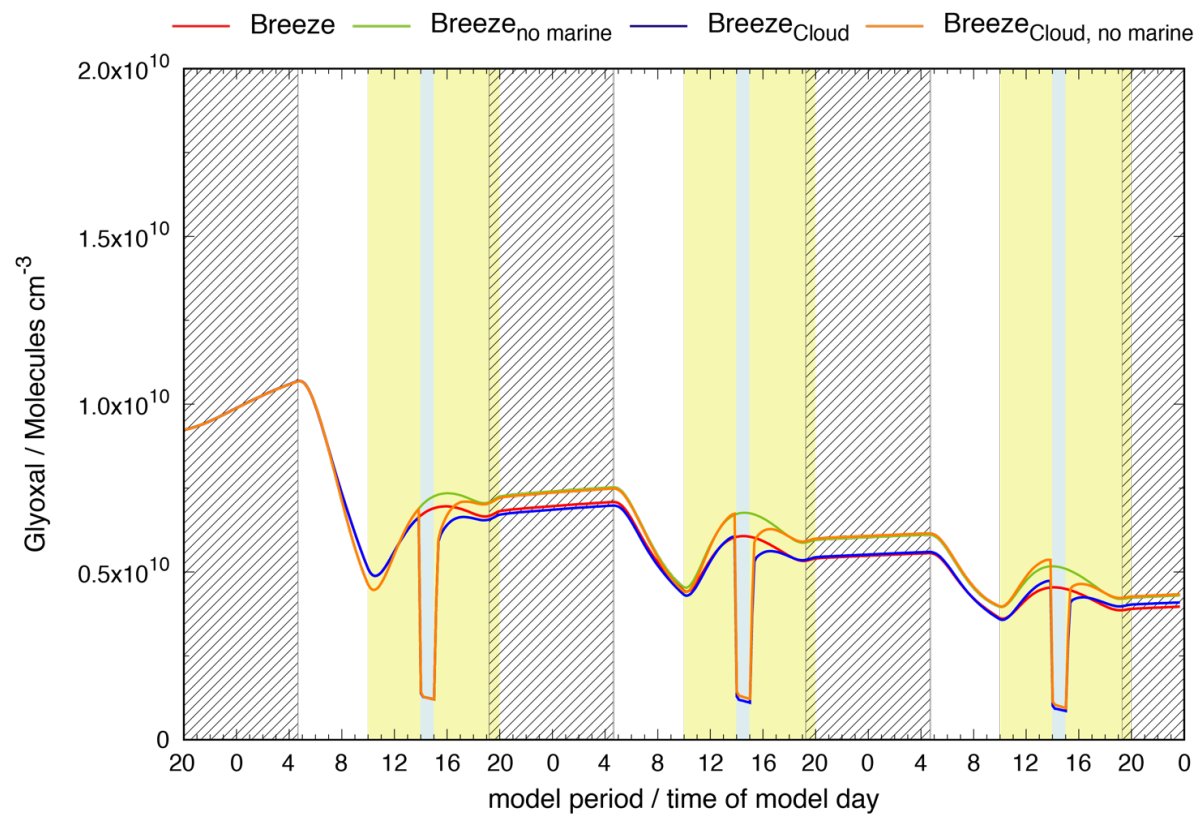

Figure S2 Modeled concentration of gaseous glyoxal during the whole modeling period. Red line:

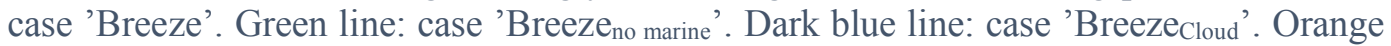

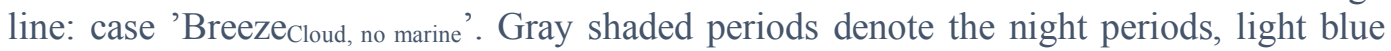
periods the cloud passage (cloud cases only), and the light-yellow periods the residence time of the air parcel over the polluted coastal site. 


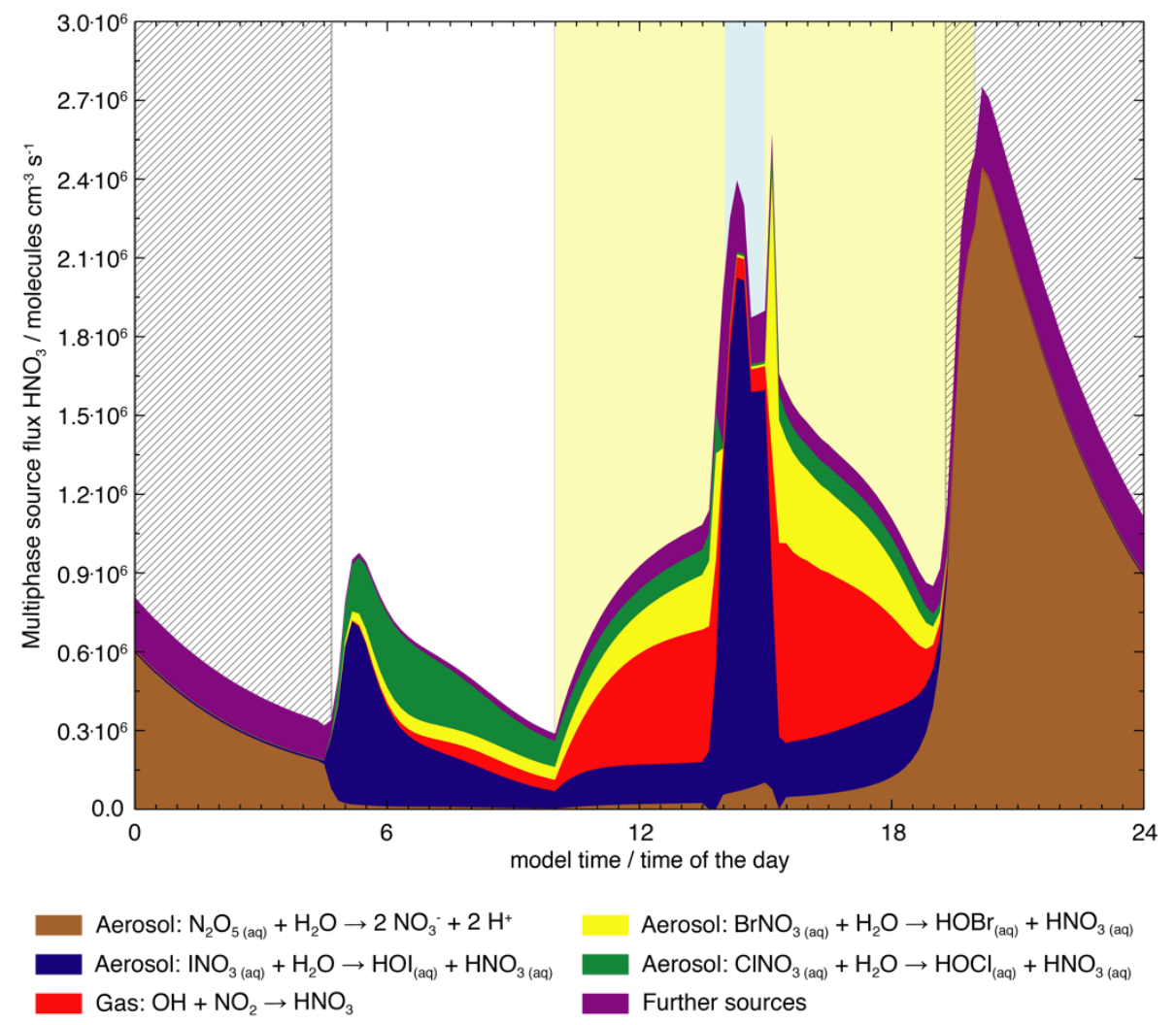

Figure S3 Modeled time-resolved source fluxes contributing to the overall nitrate formation during

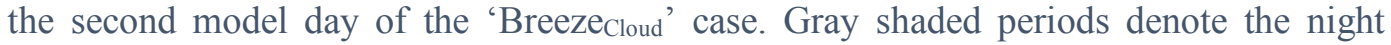
periods, light blue periods the cloud passage, and the light-yellow periods the residence time of the air parcel over the polluted coastal site.

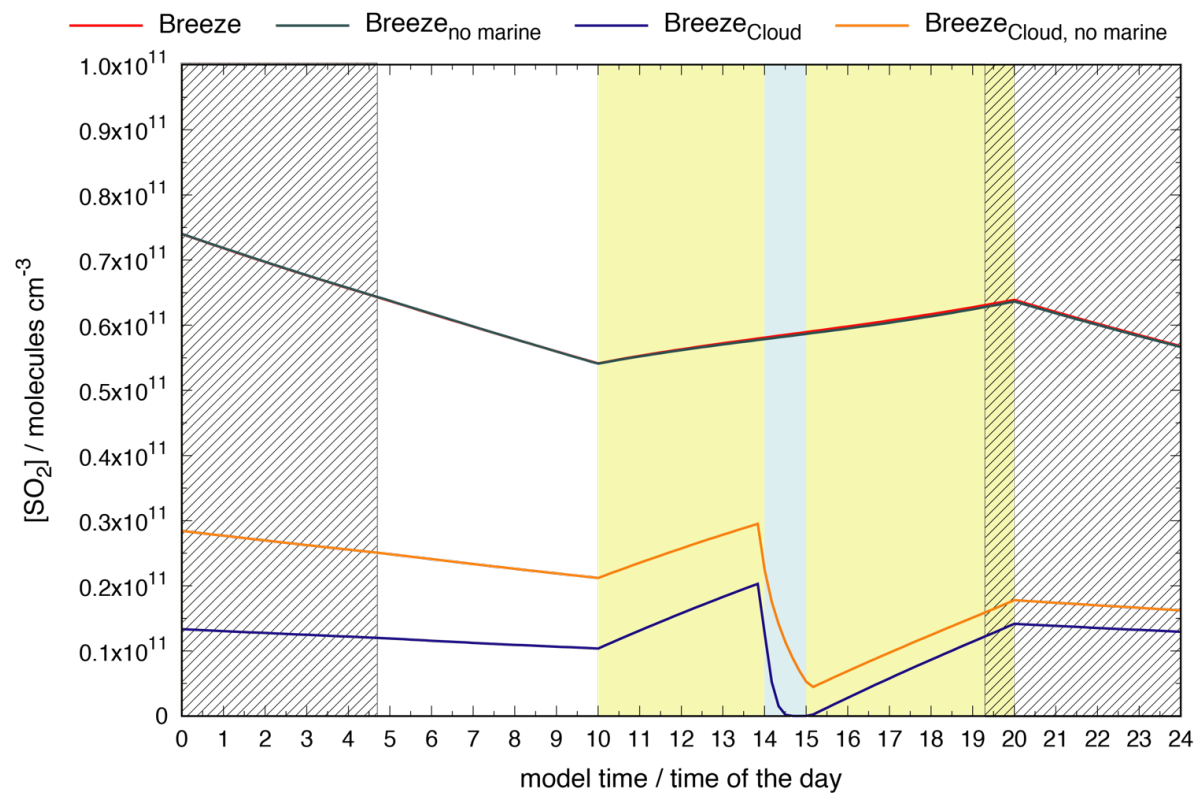

Figure S4 Modeled gas-phase $\mathrm{SO}_{2}$ concentration at the second model day. Red line: scenario 'Breeze'. Green line: scenario 'Breeze ${ }_{\text {no marine'. Blue line: scenario 'Breeze }}$ cloud'. Orange

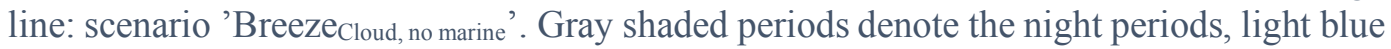
periods the cloud passage (cloud cases only), and the light-yellow periods the residence time of the air parcel over the polluted coastal site. 


\section{gas-phase reaction emission \\ aqueous-phase reaction \\ phase transfer}

production/emission

molecules $\mathrm{cm}^{-3}$
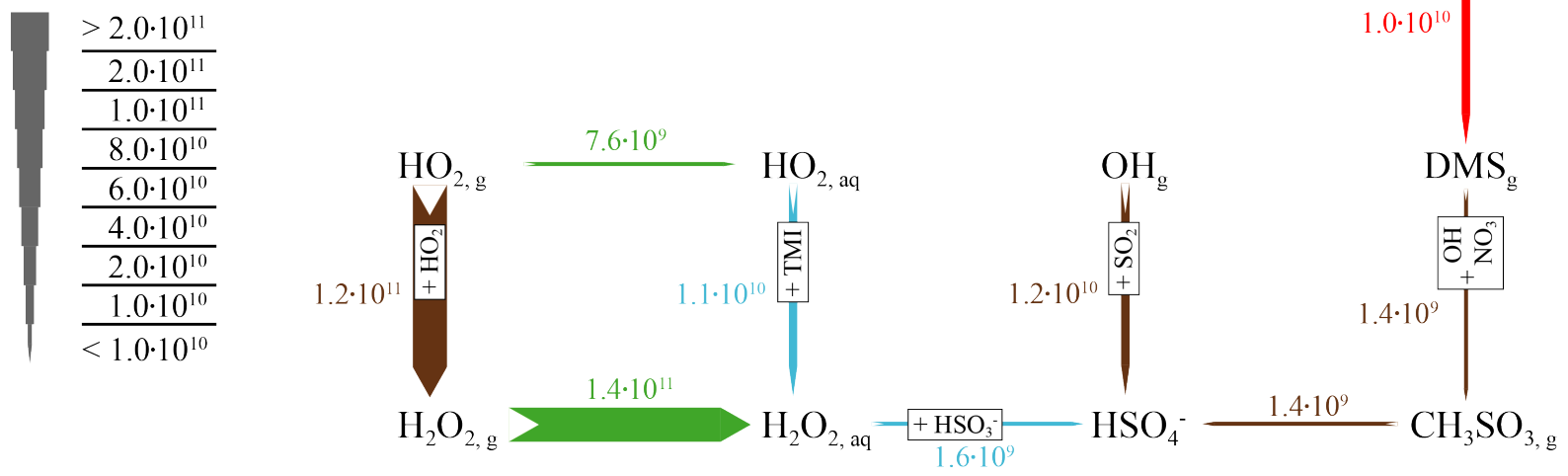

Figure S5 Scheme of multiphase source fluxes leading to the formation of sulfate over the full simulation time of the 'Breeze' case. Only oxidation fluxes exceeding 5\% of the total flux are included. The width of arrows indicates the magnitude of the mass flux. Red arrows represent emission fluxes, brown arrows represent gas-phase reactions, blue arrows aqueous-phase reactions, and green arrows phase transfer processes.
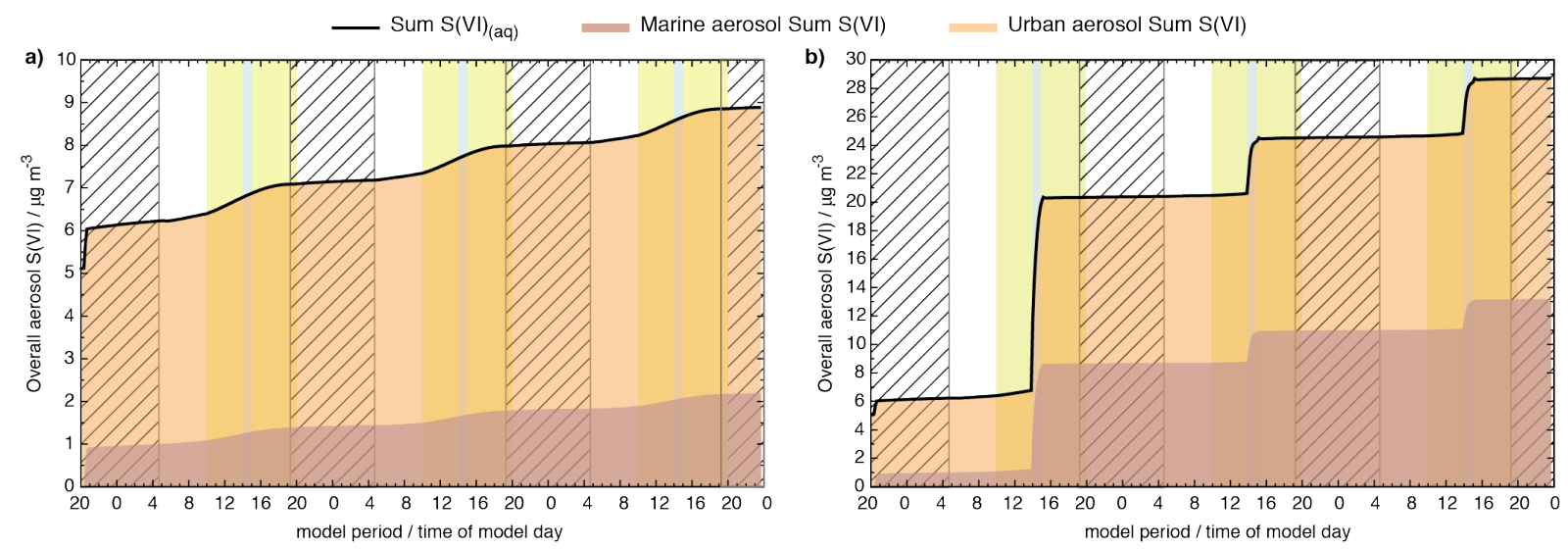

Figure S6 Modeled aqueous sulfate concentration distribution during the whole modeling period for the scenario 'Breeze' (a) and the scenario 'Breeze ${ }_{\text {Cloud }}$ (b) in $\mu \mathrm{g} \mathrm{m}^{-3}$. Black line: Overall sum of aqueous sulfate. Orange area: Sum of sulfate within the urban aerosol. Brown area: Sum of sulfate within the marine aerosol. Gray shaded periods denote the night periods, light blue periods the cloud passage (cloud cases only), and the light-yellow periods the residence time of the air parcel over the polluted coastal site. 


\section{Tables}

Table S1 Emission values of trace gases of the anthropogenic polluted continental site and the marine coastline used in the simulations.

\begin{tabular}{|c|c|c|c|c|c|}
\hline Compound & Compound String & $\begin{array}{c}\text { Continental emission rate } \\
\left(\text { molecules } \mathrm{cm}^{-3} \mathrm{~s}^{-1}\right)\end{array}$ & Ref. & $\begin{array}{c}\text { Coastal emission rate } \\
\left(\text { molecules } \mathrm{cm}^{-3} \mathrm{~s}^{-1}\right)\end{array}$ & Ref. \\
\hline \multicolumn{6}{|l|}{ Inorganics } \\
\hline $\mathrm{CO}$ & $\mathrm{CO}$ & $2.04 \mathrm{E}+07$ & & $6.15 E+06$ & 1 \\
\hline $\mathrm{NH}_{3}$ & $\mathrm{NH} 3$ & $2.32 \mathrm{E}+06$ & & $1.03 \mathrm{E}+04$ & 2 \\
\hline $\mathrm{NO}$ & NO & $4.21 \mathrm{E}+06$ & & $1.50 \mathrm{E}+04$ & 3 \\
\hline $\mathrm{NO}_{2}$ & $\mathrm{NO} 2$ & $4.61 \mathrm{E}+05$ & & - & \\
\hline $\mathrm{I}_{2}$ & $\mathrm{I} 2$ & - & & $4.00 \mathrm{E}+03$ & 3 \\
\hline \multicolumn{6}{|l|}{ Organics } \\
\hline \multicolumn{6}{|l|}{ Alkanes } \\
\hline Methane & $\mathrm{C}$ & - & & $1.76 \mathrm{E}+04$ & 4 \\
\hline n-Pentane & CCCCC & $1.61 \mathrm{E}+04$ & & - & \\
\hline i-Pentane & $\mathrm{CCC}(\mathrm{C}) \mathrm{C}$ & $2.85 \mathrm{E}+04$ & & - & \\
\hline n-Hexane & $\mathrm{CCCCCC}$ & $1.63 \mathrm{E}+04$ & & - & \\
\hline 2-Methylpentane & $\mathrm{CCCC}(\mathrm{C}) \mathrm{C}$ & $4.29 \mathrm{E}+04$ & & - & \\
\hline 3-Methylpentane & $\mathrm{CCC}(\mathrm{C}) \mathrm{CC}$ & $4.77 \mathrm{E}+03$ & & - & \\
\hline 2,2-Dimethylbutane & $\mathrm{CC}(\mathrm{C}) \mathrm{C}(\mathrm{C}) \mathrm{C}$ & $2.93 \mathrm{E}+03$ & & - & \\
\hline
\end{tabular}




\begin{tabular}{|c|c|c|c|c|c|}
\hline Compound & Compound String & $\begin{array}{l}\text { Continental emission rate } \\
\left(\text { molecules } \mathrm{cm}^{-3} \mathrm{~s}^{-1}\right)\end{array}$ & Ref. & $\begin{array}{c}\text { Coastal emission rate } \\
\left(\text { molecules } \mathrm{cm}^{-3} \mathrm{~s}^{-1}\right) \\
\end{array}$ & Ref. \\
\hline Cyclohexane & $\mathrm{C} 1 \mathrm{CCCCC} 1$ & $2.27 \mathrm{E}+04$ & & - & \\
\hline n-Heptane & $\mathrm{CCCCCCC}$ & $1.46 \mathrm{E}+05$ & & - & \\
\hline i-Heptane & $\mathrm{CCCC}(\mathrm{C}) \mathrm{CC}$ & $2.72 \mathrm{E}+04$ & & - & \\
\hline Octane & ССССССCC & $3.14 \mathrm{E}+04$ & & - & \\
\hline Nonane & СССССССCC & $1.31 \mathrm{E}+04$ & & - & \\
\hline \multicolumn{6}{|l|}{ Alkenes } \\
\hline Ethene & $\mathrm{C}=\mathrm{C}$ & $1.08 \mathrm{E}+05$ & & $2.24 \mathrm{E}+03$ & 5 \\
\hline Propene & $\mathrm{CC}=\mathrm{C}$ & $4.25 \mathrm{E}+04$ & & $8.71 \mathrm{E}+02$ & 5 \\
\hline n-Butene & $\mathrm{CCC}=\mathrm{C}$ & $9.07 \mathrm{E}+03$ & & - & \\
\hline i-Butene & $\mathrm{CC}(\mathrm{C})=\mathrm{C}$ & $2.03 \mathrm{E}+02$ & & - & \\
\hline Trimethylethylene & $\mathrm{CC}=\mathrm{C}(\mathrm{C}) \mathrm{C}$ & $4.01 \mathrm{E}+03$ & & - & \\
\hline trans-Pentene & $\mathrm{CC} \backslash \mathrm{C}=\mathrm{C} \backslash \mathrm{C}$ & $3.00 \mathrm{E}+03$ & & - & \\
\hline trans-2-Butene & $\mathrm{CC}=\mathrm{CC}$ & $2.51 \mathrm{E}+03$ & & - & \\
\hline cis-2-Butene & $\mathrm{cCC}=\mathrm{CC}$ & $2.27 \mathrm{E}+03$ & & - & \\
\hline cis-Pentene & $\mathrm{CC} / \mathrm{C}=\mathrm{C} \backslash \mathrm{C}$ & $1.84 \mathrm{E}+03$ & & - & \\
\hline cis-Hexene & $\mathrm{CCC} / \mathrm{C}=\mathrm{C} \backslash \mathrm{C}$ & $9.18 \mathrm{E}+02$ & & - & \\
\hline Acetylene & $\mathrm{C} \# \mathrm{C}$ & $2.96 \mathrm{E}+04$ & & $1.00 \mathrm{E}+02$ & 5 \\
\hline \multicolumn{6}{|l|}{ Dialkenes } \\
\hline Butadiene & $\mathrm{C}=\mathrm{CC}=\mathrm{C}$ & $8.46 \mathrm{E}+03$ & & - & \\
\hline Isoprene & $\mathrm{C}=\mathrm{CC}(\mathrm{C})=\mathrm{C}$ & $4.41 \mathrm{E}+04$ & & $1.41 \mathrm{E}+03$ & 6 \\
\hline
\end{tabular}




\begin{tabular}{|c|c|c|c|c|c|}
\hline Compound & Compound String & $\begin{array}{c}\text { Continental emission rate } \\
\left(\text { molecules } \mathrm{cm}^{-3} \mathrm{~s}^{-1}\right)\end{array}$ & Ref. & $\begin{array}{c}\text { Coastal emission rate } \\
\left(\text { molecules } \mathrm{cm}^{-3} \mathrm{~s}^{-1}\right) \\
\end{array}$ & $\overline{\text { Ref. }}$ \\
\hline \multicolumn{6}{|l|}{ Aromatics } \\
\hline Benzene & $\operatorname{clcccc} 1$ & $4.18 \mathrm{E}+03$ & & - & \\
\hline Toluene & $\operatorname{clcccc} 1 \mathrm{C}$ & $3.15 \mathrm{E}+04$ & & - & \\
\hline Ethyl benzene & $\mathrm{c} 1 \mathrm{ccccc} 1 \mathrm{CC}$ & $3.44 \mathrm{E}+02$ & & - & \\
\hline o-Xylene & CelcecclC & $2.47 \mathrm{E}+03$ & & - & \\
\hline p-Xylene & $\operatorname{clcc}(\mathrm{C}) \operatorname{ccc} 1 \mathrm{C}$ & $3.32 \mathrm{E}+03$ & & - & \\
\hline 1,2,4-Trimethylbenzene & $\mathrm{Cc} 1 \mathrm{ccc}(\mathrm{C}) \mathrm{cc} 1 \mathrm{C}$ & $2.12 \mathrm{E}+03$ & & - & \\
\hline 1,3,5-Trimethylbenzene & $\operatorname{clc}(\mathrm{C}) \operatorname{cc}(\mathrm{C}) \mathrm{cc} 1 \mathrm{C}$ & $1.95 \mathrm{E}+03$ & & - & \\
\hline m-Xylene & $\operatorname{clc}(\mathrm{C}) \operatorname{cccc} 1 \mathrm{C}$ & $9.05 \mathrm{E}+02$ & & - & \\
\hline$\alpha$-Pinene & $\mathrm{C} 12 \mathrm{CC}(\mathrm{C} 1(\mathrm{C}) \mathrm{C}) \mathrm{CC}=\mathrm{C} 2 \mathrm{C}$ & $1.05 \mathrm{E}+04$ & & $1.53 \mathrm{E}+01$ & 7 \\
\hline$\beta$-Pinene & $\mathrm{CC} 1(\mathrm{C}) \mathrm{C}(\mathrm{CC} 12) \mathrm{CCC} 2=\mathrm{C}$ & $7.45 \mathrm{E}+03$ & & - & \\
\hline Limonene & $\mathrm{C} 1 \mathrm{CC}(\mathrm{C})=\mathrm{CCC} 1 \mathrm{C}(\mathrm{C})=\mathrm{C}$ & $8.51 \mathrm{E}+03$ & & - & \\
\hline \multicolumn{6}{|l|}{ Alcohols } \\
\hline Methanol & $\mathrm{OC}$ & $2.12 \mathrm{E}+03$ & & $1.87 \mathrm{E}+05$ & 8 \\
\hline Ethanol & $\mathrm{CCO}$ & $1.07 \mathrm{E}+05$ & & $6.11 \mathrm{E}+03$ & 9 \\
\hline Propanol & $\mathrm{CCCO}$ & $1.03 \mathrm{E}+02$ & & - & \\
\hline i-Propanol & $\mathrm{CC}(\mathrm{O}) \mathrm{C}$ & $2.78 \mathrm{E}+04$ & & - & \\
\hline Butanol & $\mathrm{CCCCO}$ & $5.68 \mathrm{E}+02$ & & - & \\
\hline
\end{tabular}




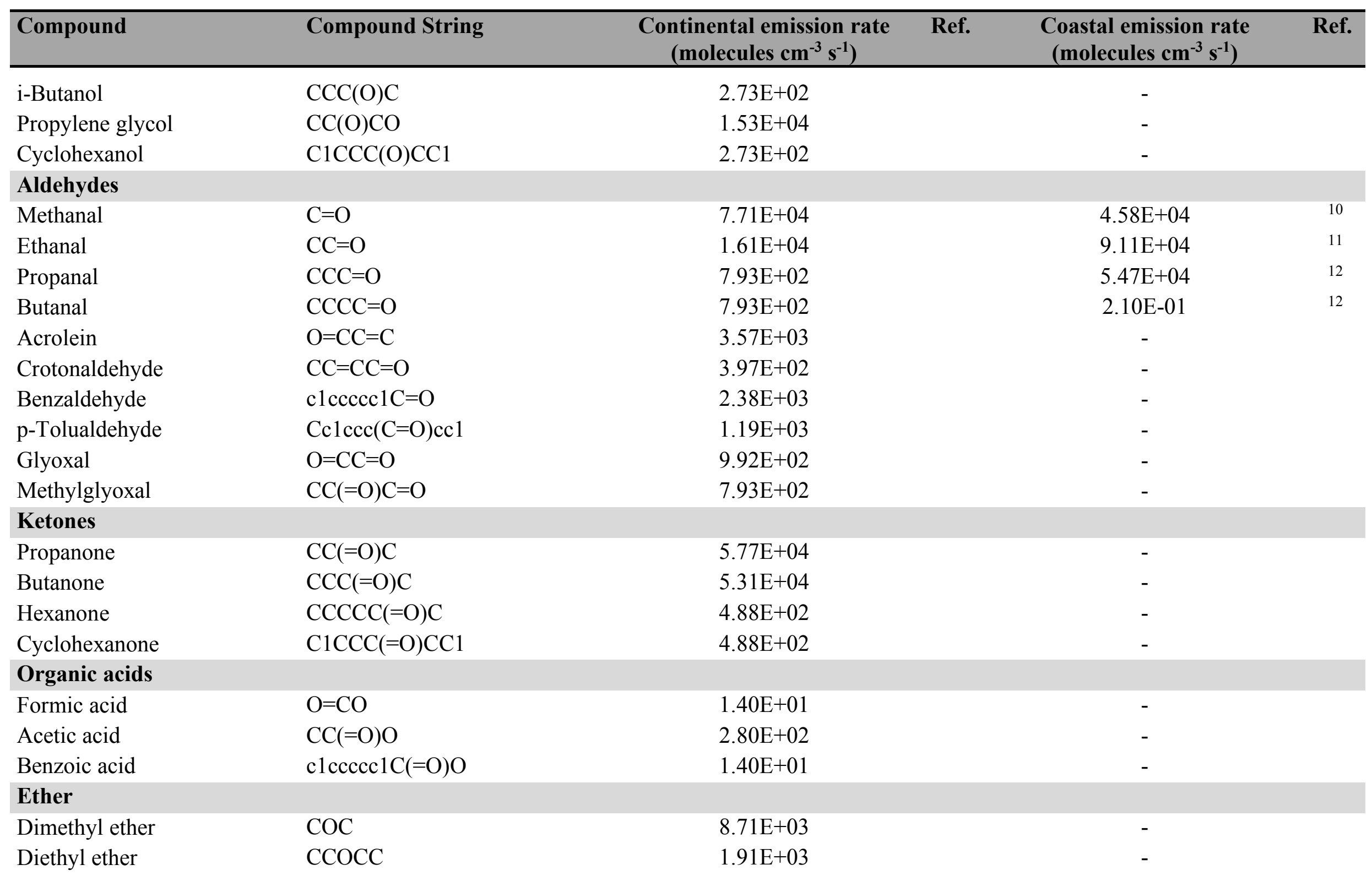




\begin{tabular}{|c|c|c|c|c|c|}
\hline Compound & Compound String & $\begin{array}{c}\text { Continental emission rate } \\
\left(\text { molecules } \mathrm{cm}^{-3} \mathrm{~s}^{-1}\right) \\
\end{array}$ & Ref. & $\begin{array}{c}\text { Coastal emission rate } \\
\left(\text { molecules } \mathrm{cm}^{-3} \mathrm{~s}^{-1}\right)\end{array}$ & Ref. \\
\hline Methyl glycol & $\mathrm{COCCO}$ & $2.79 \mathrm{E}+04$ & & - & \\
\hline Ethylene oxide & $\mathrm{C} 1 \mathrm{OC} 1$ & $2.65 \mathrm{E}+02$ & & - & \\
\hline Methyl acetate & $\mathrm{CC}(=\mathrm{O}) \mathrm{OC}$ & $7.94 \mathrm{E}+02$ & & - & \\
\hline Ethyl ethanoate & $\operatorname{CCOC}(=\mathrm{O}) \mathrm{C}$ & $1.80 \mathrm{E}+03$ & & - & \\
\hline Propyl acetate & $\operatorname{CcCOC}(=\mathrm{O}) \mathrm{C}$ & $2.58 \mathrm{E}+03$ & & - & \\
\hline \multicolumn{6}{|c|}{ Chlorinated organics } \\
\hline Chloromethane & CCL & - & & $9.10 \mathrm{E}+02$ & 12 \\
\hline Dichloromethane & CLCCL & - & & $3.20 \mathrm{E}+02$ & 12 \\
\hline Trichloromethane & CLC(CL)CL & - & & $6.40 \mathrm{E}+02$ & 12 \\
\hline 1,2-Dichloroethane & CLCCCL & $6.44 \mathrm{E}+01$ & & - & \\
\hline \multicolumn{6}{|l|}{ Brominated organics } \\
\hline Bromomethane & CBR & - & & $9.12 \mathrm{E}+01$ & 13 \\
\hline Dibromomethane & BRCBR & - & & $7.67 \mathrm{E}+02$ & 14 \\
\hline Tribromomethane & $\mathrm{BRC}(\mathrm{BR}) \mathrm{BR}$ & - & & $1.53 \mathrm{E}+03$ & 14 \\
\hline \multicolumn{6}{|l|}{ Iodinated organics } \\
\hline Iodomethane & $\mathrm{CI}$ & - & & $1.14 \mathrm{E}+03$ & 14 \\
\hline Diiodomethane & ICI & - & & $1.79 \mathrm{E}+03$ & 15 \\
\hline Ethyl iodide & ICC & - & & $5.64 \mathrm{E}+01$ & 15 \\
\hline i-Propyl iodide & $\mathrm{CC}(\mathrm{I}) \mathrm{C}$ & - & & $1.88 \mathrm{E}+01$ & 15 \\
\hline Chloroiodomethane & CLCI & - & & $9.21 \mathrm{E}+02$ & 15 \\
\hline
\end{tabular}




\begin{tabular}{|c|c|c|c|c|c|}
\hline Compound & Compound String & $\begin{array}{c}\text { Continental emission rate } \\
\left(\text { molecules } \mathrm{cm}^{-3} \mathrm{~s}^{-1}\right)\end{array}$ & Ref. & $\begin{array}{c}\text { Coastal emission rate } \\
\left(\text { molecules } \mathrm{cm}^{-3} \mathrm{~s}^{-1}\right) \\
\end{array}$ & Ref. \\
\hline Bromoiodomethane & BRCI & - & & $1.32 \mathrm{E}+02$ & 15 \\
\hline \multicolumn{6}{|l|}{ Organosulfates } \\
\hline Dimethyl sulfide & $\mathrm{CSC}$ & - & & $6.18 \mathrm{E}+04$ & 16 \\
\hline
\end{tabular}


Table S2 Initial concentration of trace gases at the beginning of the simulations.

\begin{tabular}{|c|c|c|c|c|}
\hline Compound & Compound String & $\begin{array}{c}\text { Initial concentration } \\
\left(\text { molecules } \mathrm{cm}^{-3}\right)\end{array}$ & Comments & Reference \\
\hline \multicolumn{5}{|l|}{ Inorganics } \\
\hline $\mathrm{NO}_{2}$ & & $1.15 \mathrm{E}+11$ & Old CAPRAM value & 17 \\
\hline $\mathrm{HNO}_{3}$ & & $2.55 \mathrm{E}+10$ & Old CAPRAM value & 17 \\
\hline $\mathrm{H}_{2} \mathrm{O}_{2}$ & & $2.55 \mathrm{E}+10$ & Old CAPRAM value & 17 \\
\hline $\mathrm{CO}_{2}$ & & $1.02 \mathrm{E}+16$ & Global value & \\
\hline $\mathrm{NH}_{3}$ & & $1.28 \mathrm{E}+11$ & Old CAPRAM value & 17 \\
\hline $\mathrm{HCl}$ & & $5.10 \mathrm{E}+09$ & Old CAPRAM value & 17 \\
\hline $\mathrm{SO}_{2}$ & & $1.28 \mathrm{E}+11$ & Old CAPRAM value & 17 \\
\hline \multicolumn{5}{|l|}{ Organics } \\
\hline Propane & $\mathrm{CCC}$ & $9.93 \mathrm{E}+10$ & Measurement average & \\
\hline n-Butane & $\mathrm{CCCC}$ & $8.14 \mathrm{E}+10$ & Measurement average & \\
\hline i-Butene & $\mathrm{CC}(\mathrm{C}) \mathrm{C}$ & $3.91 \mathrm{E}+10$ & Measurement average & \\
\hline n-Pentane & $\mathrm{CCCCC}$ & $2.55 \mathrm{E}+10$ & Measurement average & \\
\hline i-Pentane & $\mathrm{CCC}(\mathrm{C}) \mathrm{C}$ & $6.61 \mathrm{E}+10$ & Measurement average & \\
\hline n-Hexane & $\mathrm{CCCCCC}$ & $1.92 \mathrm{E}+10$ & Measurement average & \\
\hline 2-Methylpentane & $\operatorname{CCCC}(\mathrm{C}) \mathrm{C}$ & $1.12 \mathrm{E}+10$ & Measurement average & \\
\hline
\end{tabular}




\begin{tabular}{|c|c|c|c|}
\hline Compound & Compound String & $\begin{array}{c}\text { Initial concentration } \\
\left(\text { molecules } \mathrm{cm}^{-3}\right)\end{array}$ & Comments \\
\hline 3-Methylpentane & $\mathrm{CCC}(\mathrm{C}) \mathrm{CC}$ & $1.05 \mathrm{E}+10$ & Measurement average \\
\hline n-Heptane & $\mathrm{CCCCCCC}$ & $7.73 \mathrm{E}+09$ & Measurement average \\
\hline Octane & $\mathrm{CCCCCCCC}$ & $3.87 \mathrm{E}+09$ & Measurement average \\
\hline Nonane & $\mathrm{CCCCCCCCC}$ & $6.81 \mathrm{E}+09$ & Measurement average \\
\hline Decane & $\mathrm{CCCCCCCCCC}$ & $1.15 \mathrm{E}+10$ & Measurement average \\
\hline Undecane & $\mathrm{CCCCCCCCCCC}$ & $9.05 \mathrm{E}+09$ & Measurement average \\
\hline Dodecane & СCСССCCCCCCC & $7.81 \mathrm{E}+09$ & Measurement average \\
\hline \multicolumn{4}{|l|}{ Alkenes } \\
\hline Ethene & $\mathrm{C}=\mathrm{C}$ & $7.83 \mathrm{E}+10$ & Measurement average \\
\hline Propene & $\mathrm{CC}=\mathrm{C}$ & $3.64 \mathrm{E}+10$ & Measurement average \\
\hline n-Butene & $\mathrm{CCC}=\mathrm{C}$ & $1.57 \mathrm{E}+10$ & Measurement average \\
\hline cis-2-Butene & $\mathrm{cCC}=\mathrm{CC}$ & $6.15 \mathrm{E}+09$ & Measurement average \\
\hline trans-2-Butene & $\mathrm{CC}=\mathrm{CC}$ & $6.66 \mathrm{E}+09$ & Measurement average \\
\hline n-Pentene & $\mathrm{CCCC}=\mathrm{C}$ & $3.49 \mathrm{E}+09$ & Measurement average \\
\hline trans-Pentene & $\mathrm{CC} / \mathrm{C}=\mathrm{C} \backslash \mathrm{C}$ & $3.04 \mathrm{E}+09$ & Measurement average \\
\hline cis-Pentene & $\mathrm{CC} \backslash \mathrm{C}=\mathrm{C} \backslash \mathrm{C}$ & $4.01 \mathrm{E}+09$ & Measurement average \\
\hline Acetylene & $\mathrm{C} \# \mathrm{C}$ & $4.86 \mathrm{E}+10$ & Measurement average \\
\hline \multicolumn{4}{|l|}{ Dialkenes } \\
\hline Butadiene & $\mathrm{C}=\mathrm{CC}=\mathrm{C}$ & $1.13 \mathrm{E}+10$ & Measurement average \\
\hline Isoprene & $\mathrm{C}=\mathrm{CC}(\mathrm{C})=\mathrm{C}$ & $1.05 \mathrm{E}+10$ & Measurement average \\
\hline \multicolumn{4}{|l|}{ Aromatics } \\
\hline Benzene & $\operatorname{clcccc} 1$ & $1.57 \mathrm{E}+10$ & Measurement average \\
\hline Styrene & $\operatorname{c} 1 \operatorname{cccc} 1 \mathrm{C}=\mathrm{C}$ & $2.65 \mathrm{E}+09$ & Measurement average \\
\hline Toluene & $\operatorname{clcccc} 1 \mathrm{C}$ & $5.45 \mathrm{E}+10$ & Measurement average \\
\hline Ethyl benzene & $\operatorname{clcccc} 1 \mathrm{CC}$ & $1.04 \mathrm{E}+10$ & Measurement average \\
\hline n-Propyl benzene & $\mathrm{c} 1 \mathrm{cccc} 1 \mathrm{CCC}$ & $3.89 \mathrm{E}+09$ & Measurement average \\
\hline
\end{tabular}




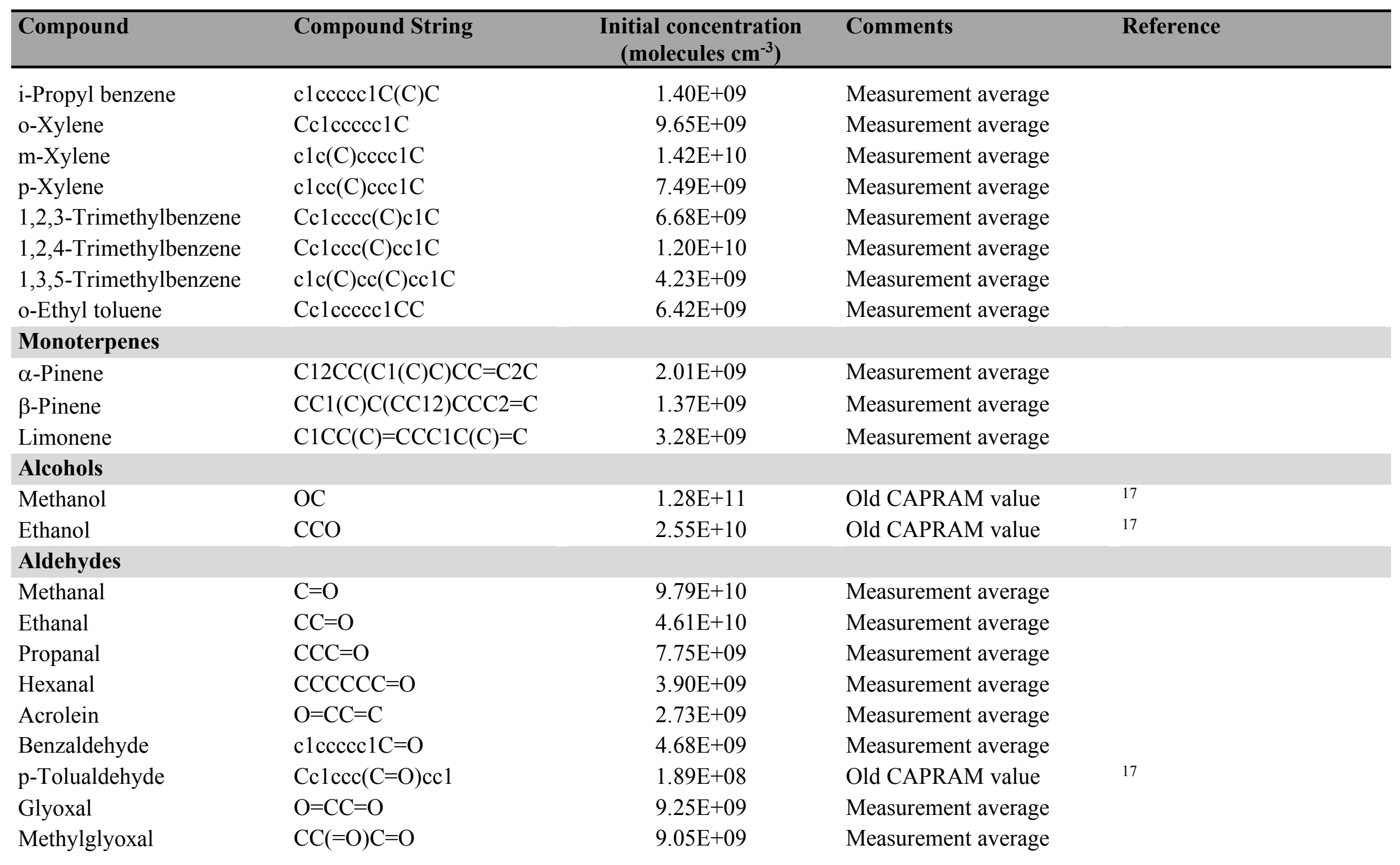




\begin{tabular}{|c|c|c|c|c|}
\hline Compound & Compound String & $\begin{array}{c}\text { Initial concentration } \\
\left(\text { molecules } \mathrm{cm}^{-3}\right)\end{array}$ & Comments & Reference \\
\hline \multicolumn{5}{|l|}{ Ketones } \\
\hline Acetone & $\mathrm{CC}(=\mathrm{O}) \mathrm{C}$ & $5.79 \mathrm{E}+10$ & Measurement average & \\
\hline Methylvinylketone & $\mathrm{CCC}(=\mathrm{O}) \mathrm{C}$ & $1.40 \mathrm{E}+10$ & Measurement average & \\
\hline \multicolumn{5}{|c|}{ Organic hydroperoxides } \\
\hline Methyl hydroperoxide & $\mathrm{COO}$ & $2.55 \mathrm{E}+08$ & Old CAPRAM value & 17 \\
\hline PAN & $\mathrm{CC}(=\mathrm{O}) \mathrm{OON}(=\mathrm{O})=\mathrm{O}$ & $2.55 \mathrm{E}+08$ & Old CAPRAM value & 17 \\
\hline \multicolumn{5}{|l|}{ Chlorinated organics } \\
\hline Chloromethane & CCL & $2.38 \mathrm{E}+10$ & Old CAPRAM value & 17 \\
\hline Dichloromethane & CLCCL & $4.00 \mathrm{E}+09$ & Old CAPRAM value & 17 \\
\hline Trichloromethane & CLC(CL)CL & $6.67 \mathrm{E}+08$ & Old CAPRAM value & 17 \\
\hline Tribromomethane & $\mathrm{BRC}(\mathrm{BR}) \mathrm{BR}$ & $3.25 \mathrm{E}+08$ & Old CAPRAM value & 17 \\
\hline $\begin{array}{l}\text { Ait-Helal et al. }{ }^{18,} \text { Bake } \\
\text { 27, Masiol et al. }{ }^{28,} \text { Okac } \\
\text { Schneidemesser et al. }{ }^{3}\end{array}$ & $\begin{array}{l}{ }^{19,} \text { Cahill }{ }^{20,} \text { Derwent } \\
{ }^{29} \text {, Paralovo et al. }{ }^{30,}\end{array}$ & $\begin{array}{l}\mathrm{t} \text { al. }{ }^{22,} \text { Fuselli } \\
\text { rez et al. }{ }^{32,} \mathrm{Ra}\end{array}$ & $\begin{array}{l}{ }^{23,} \text { Hoshi et al. }{ }^{24,} \text { Kunta } \\
\text { et al. }{ }^{33,} \text {, Roukos et al. }{ }^{3 .}\end{array}$ & $\begin{array}{l}{ }^{25,} \text { Lerner et al. }{ }^{26,} \text { Liu et al. } \\
\text { tal. }{ }^{35,} \text { Sturaro et al. }{ }^{36,} \text {, von }\end{array}$ \\
\hline
\end{tabular}


Table S3 Implemented deposition values during the simulations. Values are scaled for a boundary layer height of $1000 \mathrm{~m}$.

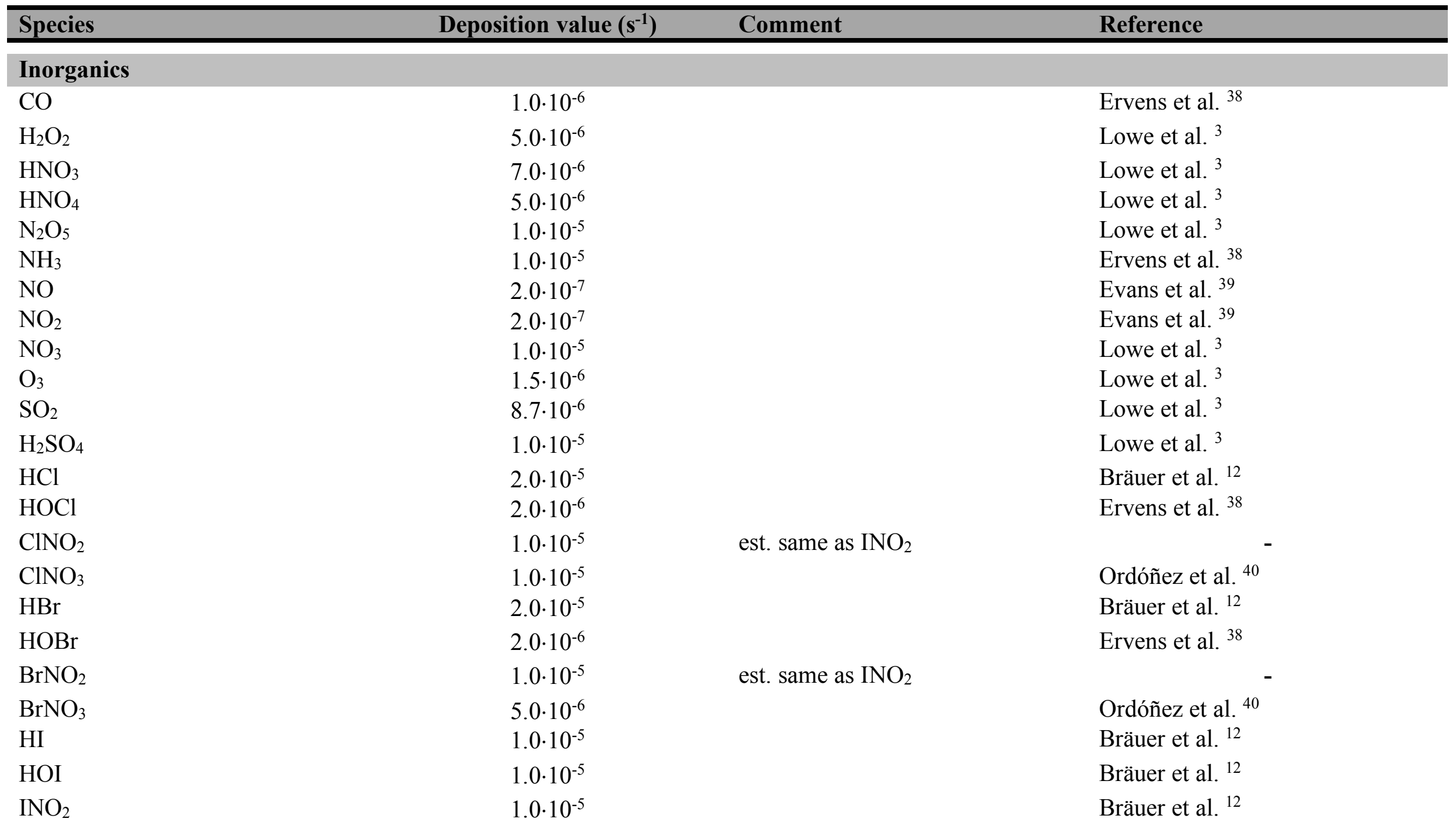




\begin{tabular}{|c|c|c|c|}
\hline Species & Deposition value $\left(\mathrm{s}^{-1}\right)$ & Comment & Reference \\
\hline $\mathrm{INO}_{3}$ & $1.0 \cdot 10^{-5}$ & & Bräuer et al. ${ }^{12}$ \\
\hline $\mathrm{I}_{2} \mathrm{O}_{2}$ & $1.0 \cdot 10^{-5}$ & & Saiz-Lopez et al. ${ }^{41}$ \\
\hline $\mathrm{I}_{2} \mathrm{O}_{4}$ & $1.0 \cdot 10^{-5}$ & & Saiz-Lopez et al. ${ }^{41}$ \\
\hline \multicolumn{4}{|l|}{ Organics } \\
\hline Formaldehyde & $5.0 \cdot 10^{-6}$ & & Ervens et al. ${ }^{38}$ \\
\hline Methyl Hydroperoxide & $2.5 \cdot 10^{-6}$ & & Evans et al. ${ }^{39}$ \\
\hline PAN & $1.0 \cdot 10^{-7}$ & & Evans et al. ${ }^{39}$ \\
\hline Formic acid & $1.0 \cdot 10^{-5}$ & & Lowe et al. ${ }^{3}$ \\
\hline Dimethyl sulfoxide & $5.0 \cdot 10^{-6}$ & & Hoffmann et al. ${ }^{42}$ \\
\hline
\end{tabular}


Table S4 Gas-phase reactions implemented within the CAPRAM-HM3.0. For the sake of clarity organic species are indicated by SMILES nomenclature.

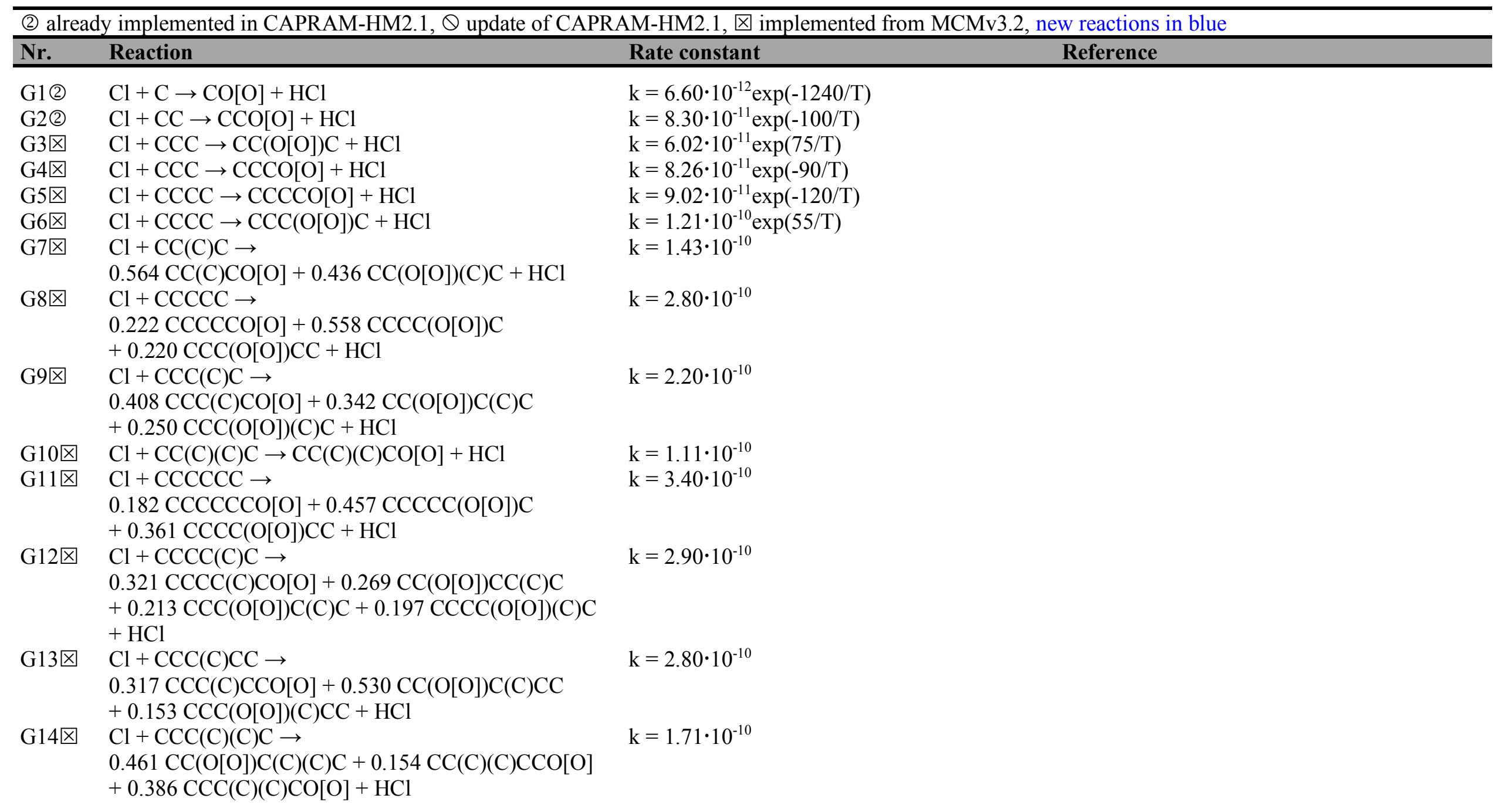




\begin{tabular}{|c|c|c|c|}
\hline Nr. & Reaction & Rate constant & Reference \\
\hline G15区 & $\begin{array}{l}\mathrm{Cl}+\mathrm{CC}(\mathrm{C}) \mathrm{C}(\mathrm{C}) \mathrm{C} \rightarrow \\
0.478 \mathrm{CC}(\mathrm{O}[\mathrm{O}])(\mathrm{C}) \mathrm{C}(\mathrm{C}) \mathrm{C}+0.522 \mathrm{CC}(\mathrm{C}) \mathrm{C}(\mathrm{C}) \mathrm{CO}[\mathrm{O}] \\
+\mathrm{HCl}\end{array}$ & $\mathrm{k}=2.30 \cdot 10^{-10}$ & \\
\hline G16凶 & $\mathrm{Cl}+\mathrm{CCCCCCC} \rightarrow \mathrm{CCCCC}(\mathrm{O}[\mathrm{O}]) \mathrm{CC}+\mathrm{HCl}$ & $\mathrm{k}=3.90 \cdot 10^{-10}$ & \\
\hline G17凶 & $\begin{array}{l}\mathrm{Cl}+\mathrm{CCCCC}(\mathrm{C}) \mathrm{C} \rightarrow \\
0.779 \mathrm{CC}(\mathrm{O}[\mathrm{O}]) \mathrm{CCC}(\mathrm{C}) \mathrm{C}+0.221 \mathrm{CCCCC}(\mathrm{O}[\mathrm{O}])(\mathrm{C}) \mathrm{C} \\
+\mathrm{HCl}\end{array}$ & $\mathrm{k}=3.50 \cdot 10^{-10}$ & \\
\hline G18凶 & $\begin{array}{l}\mathrm{Cl}+\mathrm{CCCC}(\mathrm{C}) \mathrm{CC} \rightarrow \\
0.793 \mathrm{CC}(\mathrm{O}[\mathrm{O}]) \mathrm{CC}(\mathrm{C}) \mathrm{CC}+0.207 \mathrm{CCCC}(\mathrm{O}[\mathrm{O}])(\mathrm{C}) \mathrm{CC} \\
+\mathrm{HCl}\end{array}$ & $\mathrm{k}=3.11 \cdot 10^{-10}$ & \\
\hline G19凶 & $\mathrm{Cl}+\mathrm{CCCCCCCC} \rightarrow \mathrm{CCCCCC}(\mathrm{O}[\mathrm{O}]) \mathrm{CC}+\mathrm{HCl}$ & $\mathrm{k}=4.60 \cdot 10^{-10}$ & \\
\hline $\mathrm{G} 20 \overline{\mathrm{Q}}$ & $\mathrm{Cl}+\mathrm{CCCCCCCCC} \rightarrow \mathrm{CCCCCCC}(\mathrm{O}[\mathrm{O}]) \mathrm{CC}+\mathrm{HCl}$ & $\mathrm{k}=4.80 \cdot 10^{-10}$ & \\
\hline G21凶 & $\mathrm{Cl}+\mathrm{CCCCCCCCCC} \rightarrow \mathrm{CCCCCCCC}(\mathrm{O}[\mathrm{O}]) \mathrm{CC}+\mathrm{HCl}$ & $\mathrm{k}=5.55 \cdot 10^{-10}$ & \\
\hline G22区 & $\begin{array}{l}\mathrm{Cl}+\mathrm{CCCCCCCCCCC} \rightarrow \\
\mathrm{CCCCCCCCC}(\mathrm{O}[\mathrm{O}]) \mathrm{CC}+\mathrm{HCl}\end{array}$ & $\mathrm{k}=6.17 \cdot 10^{-10}$ & \\
\hline G23区 & $\begin{array}{l}\mathrm{Cl}+\mathrm{CCCCCCCCCCCC} \rightarrow \\
\mathrm{CCCCCCСCC}(\mathrm{O}[\mathrm{O}]) \mathrm{CC}+\mathrm{HCl}\end{array}$ & $\mathrm{k}=6.74 \cdot 10^{-10}$ & \\
\hline G24区 & $\mathrm{Cl}+\mathrm{C} 1 \mathrm{CCCCC} 1 \rightarrow \mathrm{C} 1 \mathrm{CCC}(\mathrm{O}[\mathrm{O}]) \mathrm{CC} 1+\mathrm{HCl}$ & $\mathrm{k}=3.50 \cdot 10^{-10}$ & \\
\hline $\mathrm{G} 25(2)$ & $\mathrm{CCl}+\mathrm{OH} \rightarrow \mathrm{ClCO}[\mathrm{O}]+\mathrm{H}_{2} \mathrm{O}$ & $\mathrm{k}=7.33 \cdot 10^{-18 *} \mathrm{~T}^{2} \exp (-809 / \mathrm{T})$ & \\
\hline G26(2) & $\mathrm{ClCCl}+\mathrm{OH} \rightarrow[\mathrm{O}] \mathrm{OC}(\mathrm{Cl}) \mathrm{Cl}+\mathrm{H}_{2} \mathrm{O}$ & $\mathrm{k}=6.14 \cdot 10^{-18 *} \mathrm{~T}^{2} \exp (-389 / \mathrm{T})$ & \\
\hline G27(2) & $\mathrm{ClC}(\mathrm{Cl}) \mathrm{Cl}+\mathrm{OH} \rightarrow[\mathrm{O}] \mathrm{OC}(\mathrm{Cl})(\mathrm{Cl}) \mathrm{Cl}+\mathrm{H}_{2} \mathrm{O}$ & $\mathrm{k}=1.80 \cdot 10^{-18 *} \mathrm{~T}^{2} \exp (-129 / \mathrm{T})$ & \\
\hline $\mathrm{G} 28(2)$ & $\mathrm{CC}(\mathrm{Cl})(\mathrm{Cl}) \mathrm{Cl}+\mathrm{OH} \rightarrow \mathrm{ClC}(\mathrm{Cl})(\mathrm{Cl}) \mathrm{CO}[\mathrm{O}]+\mathrm{H}_{2} \mathrm{O}$ & $\mathrm{k}=2.25 \cdot 10^{-18 *} \mathrm{~T}^{2} \exp (-910 / \mathrm{T})$ & \\
\hline $\mathrm{G} 29(2)$ & $\mathrm{ClC}(\mathrm{Cl})=\mathrm{C}(\mathrm{Cl}) \mathrm{Cl}+\mathrm{OH} \rightarrow \mathrm{OC}(\mathrm{Cl})(\mathrm{Cl}) \mathrm{C}(\mathrm{Cl})(\mathrm{Cl}) \mathrm{O}[\mathrm{O}]$ & $\mathrm{k}=3.50 \cdot 10^{-12} \exp (-920 / \mathrm{T})$ & \\
\hline G30(2) & $\begin{array}{l}\mathrm{ClC}(\mathrm{Cl})=\mathrm{CCl}+\mathrm{OH} \rightarrow \\
0.50 \mathrm{OC}(\mathrm{Cl}) \mathrm{C}(\mathrm{Cl})(\mathrm{Cl}) \mathrm{O}[\mathrm{O}]+ \\
0.50 \mathrm{OC}(\mathrm{Cl})(\mathrm{Cl}) \mathrm{C}(\mathrm{Cl}) \mathrm{O}[\mathrm{O}]\end{array}$ & $\mathrm{k}=3.00 \cdot 10^{-13} \exp (565 / \mathrm{T})$ & \\
\hline G31凶 & $\mathrm{Cl} / \mathrm{C}=\mathrm{ClCl}+\mathrm{OH} \rightarrow \mathrm{OC}(\mathrm{Cl}) \mathrm{C}(\mathrm{Cl}) \mathrm{O}[\mathrm{O}]$ & $\mathrm{k}=1.94 \cdot 10^{-12} \exp (90 / \mathrm{T})$ & \\
\hline G32区 & $\mathrm{Cl} / \mathrm{C}=\mathrm{C} / \mathrm{Cl}+\mathrm{OH} \rightarrow \mathrm{OC}(\mathrm{Cl}) \mathrm{C}(\mathrm{Cl}) \mathrm{O}[\mathrm{O}]$ & $\mathrm{k}=1.01 \cdot 10^{-12} \exp (250 / \mathrm{T})$ & \\
\hline G33区 & $\mathrm{ClCCCl}+\mathrm{OH} \rightarrow \mathrm{ClCC}(\mathrm{Cl}) \mathrm{O}[\mathrm{O}]+\mathrm{H}_{2} \mathrm{O}$ & $\mathrm{k}=8.69 \cdot 10^{-12} \exp (-1070 / \mathrm{T})$ & \\
\hline G34区 & $\begin{array}{l}\mathrm{ClC}(\mathrm{Cl})=\mathrm{C}+\mathrm{OH} \rightarrow \\
0.50 \mathrm{OCC}(\mathrm{Cl})(\mathrm{Cl}) \mathrm{O}[\mathrm{O}]+0.50 \mathrm{OC}(\mathrm{Cl})(\mathrm{Cl}) \mathrm{CO}[\mathrm{O}]\end{array}$ & $\mathrm{k}=2.00 \cdot 10^{-12} \exp (506 / \mathrm{T})$ & \\
\hline
\end{tabular}




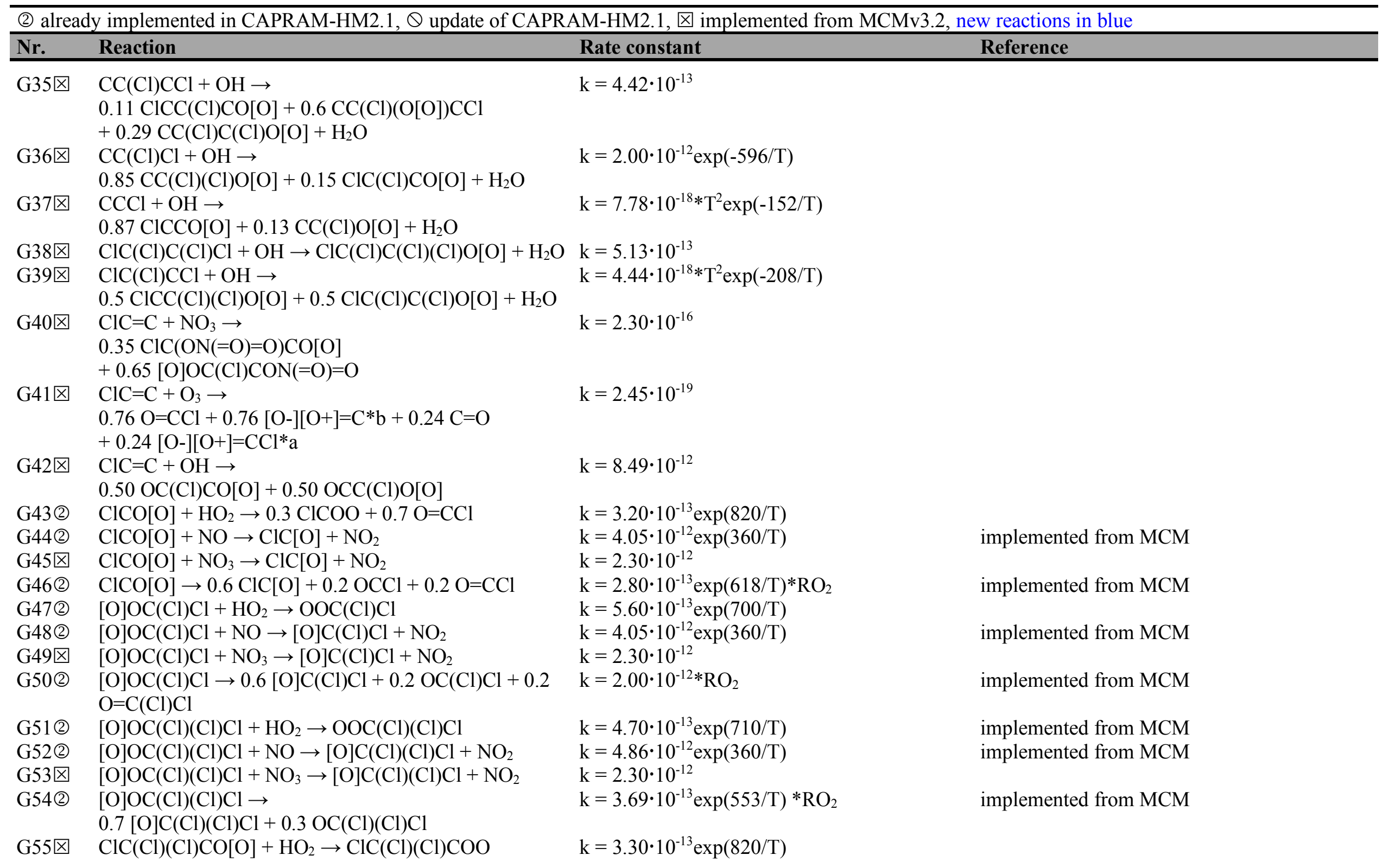




\begin{tabular}{|c|c|c|c|}
\hline Nr. & Reaction & Rate constant & Reference \\
\hline G56(2) & $\mathrm{ClC}(\mathrm{Cl})(\mathrm{Cl}) \mathrm{CO}[\mathrm{O}]+\mathrm{NO} \rightarrow \mathrm{ClC}(\mathrm{Cl})(\mathrm{Cl}) \mathrm{C}[\mathrm{O}]+\mathrm{NO}_{2}$ & $\mathrm{k}=4.05 \cdot 10^{-12} \exp (360 / \mathrm{T})$ & \\
\hline G57凶 & $\mathrm{ClC}(\mathrm{Cl})(\mathrm{Cl}) \mathrm{CO}[\mathrm{O}]+\mathrm{NO}_{3} \rightarrow \mathrm{ClC}(\mathrm{Cl})(\mathrm{Cl}) \mathrm{C}[\mathrm{O}]+\mathrm{NO}_{2}$ & $\mathrm{k}=2.30 \cdot 10^{-12}$ & \\
\hline G58(2) & $\begin{array}{l}\mathrm{ClC}(\mathrm{Cl})(\mathrm{Cl}) \mathrm{CO}[\mathrm{O}] \rightarrow \\
0.6 \mathrm{ClC}(\mathrm{Cl})(\mathrm{Cl}) \mathrm{C}[\mathrm{O}]+0.2 \mathrm{OCC}(\mathrm{Cl})(\mathrm{Cl}) \mathrm{Cl} \\
+0.2 \mathrm{ClC}(\mathrm{Cl})(\mathrm{Cl}) \mathrm{C}=\mathrm{O}\end{array}$ & $\mathrm{k}=2.00 \cdot 10^{-12} * \mathrm{RO}_{2}$ & implemented from MCM \\
\hline G59凶 & $\begin{array}{l}\mathrm{OC}(\mathrm{Cl})(\mathrm{Cl}) \mathrm{C}(\mathrm{Cl})(\mathrm{Cl}) \mathrm{O}[\mathrm{O}]+\mathrm{HO}_{2} \rightarrow \\
\mathrm{OC}(\mathrm{Cl})(\mathrm{Cl}) \mathrm{C}(\mathrm{Cl})(\mathrm{Cl}) \mathrm{OO}\end{array}$ & $\mathrm{k}=3.30 \cdot 10^{-13} \exp (820 / \mathrm{T})$ & \\
\hline G60(2) & $\begin{array}{l}\mathrm{OC}(\mathrm{Cl})(\mathrm{Cl}) \mathrm{C}(\mathrm{Cl})(\mathrm{Cl}) \mathrm{O}[\mathrm{O}]+\mathrm{NO} \rightarrow \\
\mathrm{OC}(\mathrm{Cl})(\mathrm{Cl}) \mathrm{C}(\mathrm{Cl})(\mathrm{Cl})[\mathrm{O}]+\mathrm{NO}_{2}\end{array}$ & $\mathrm{k}=4.05 \cdot 10^{-12} \exp (360 / \mathrm{T})$ & implemented from MCM \\
\hline G61凶 & $\begin{array}{l}\mathrm{OC}(\mathrm{Cl})(\mathrm{Cl}) \mathrm{C}(\mathrm{Cl})(\mathrm{Cl}) \mathrm{O}[\mathrm{O}]+\mathrm{NO}_{3} \rightarrow \\
\mathrm{OC}(\mathrm{Cl})(\mathrm{Cl}) \mathrm{C}(\mathrm{Cl})(\mathrm{Cl})[\mathrm{O}]+\mathrm{NO}_{2}\end{array}$ & $\mathrm{k}=2.30 \cdot 10^{-12}$ & \\
\hline G62(2) & $\begin{array}{l}\mathrm{OC}(\mathrm{Cl})(\mathrm{Cl}) \mathrm{C}(\mathrm{Cl})(\mathrm{Cl}) \mathrm{O}[\mathrm{O}] \rightarrow \\
0.3 \mathrm{OC}(\mathrm{Cl})(\mathrm{Cl}) \mathrm{C}(\mathrm{O})(\mathrm{Cl}) \mathrm{Cl} \\
+0.7 \mathrm{OC}(\mathrm{Cl})(\mathrm{Cl}) \mathrm{C}(\mathrm{Cl})(\mathrm{Cl})[\mathrm{O}]\end{array}$ & $\mathrm{k}=9.20 \cdot 10^{-14} * \mathrm{RO}_{2}$ & implemented from MCM \\
\hline G63凶 & $\mathrm{OC}(\mathrm{Cl}) \mathrm{C}(\mathrm{Cl})(\mathrm{Cl}) \mathrm{O}[\mathrm{O}]+\mathrm{HO}_{2} \rightarrow \mathrm{OC}(\mathrm{Cl}) \mathrm{C}(\mathrm{Cl})(\mathrm{Cl}) \mathrm{OO}$ & $\mathrm{k}=3.30 \cdot 10^{-13} \exp (820 / \mathrm{T})$ & \\
\hline G64(2) & $\begin{array}{l}\mathrm{OC}(\mathrm{Cl}) \mathrm{C}(\mathrm{Cl})(\mathrm{Cl}) \mathrm{O}[\mathrm{O}]+\mathrm{NO} \rightarrow \\
\mathrm{OC}(\mathrm{Cl}) \mathrm{C}(\mathrm{Cl})(\mathrm{Cl})[\mathrm{O}]+\mathrm{NO}_{2}\end{array}$ & $\mathrm{k}=4.05 \cdot 10^{-12} \exp (360 / \mathrm{T})$ & implemented from MCM \\
\hline G65区 & $\begin{array}{l}\mathrm{OC}(\mathrm{Cl}) \mathrm{C}(\mathrm{Cl})(\mathrm{Cl}) \mathrm{O}[\mathrm{O}]+\mathrm{NO}_{3} \rightarrow \\
\mathrm{OC}(\mathrm{Cl}) \mathrm{C}(\mathrm{Cl})(\mathrm{Cl})[\mathrm{O}]+\mathrm{NO}_{2}\end{array}$ & $\mathrm{k}=2.30 \cdot 10^{-12}$ & \\
\hline G662(2) & $\begin{array}{l}\mathrm{OC}(\mathrm{Cl}) \mathrm{C}(\mathrm{Cl})(\mathrm{Cl}) \mathrm{O}[\mathrm{O}] \rightarrow \\
0.3 \mathrm{OC}(\mathrm{Cl})(\mathrm{Cl}) \mathrm{C}(\mathrm{O}) \mathrm{Cl}+0.7 \mathrm{OC}(\mathrm{Cl}) \mathrm{C}(\mathrm{Cl})(\mathrm{Cl})[\mathrm{O}]\end{array}$ & $\mathrm{k}=9.20 \cdot 10^{-14} * \mathrm{RO}_{2}$ & implemented from MCM \\
\hline G67凶 & $\mathrm{OC}(\mathrm{Cl})(\mathrm{Cl}) \mathrm{C}(\mathrm{Cl}) \mathrm{O}[\mathrm{O}]+\mathrm{HO}_{2} \rightarrow \mathrm{OC}(\mathrm{Cl})(\mathrm{Cl}) \mathrm{C}(\mathrm{Cl}) \mathrm{OO}$ & $\mathrm{k}=3.30 \cdot 10^{-13} \exp (820 / \mathrm{T})$ & \\
\hline G68(2) & $\begin{array}{l}\mathrm{OC}(\mathrm{Cl})(\mathrm{Cl}) \mathrm{C}(\mathrm{Cl}) \mathrm{O}[\mathrm{O}]+\mathrm{NO} \rightarrow \\
\mathrm{OC}(\mathrm{Cl})(\mathrm{Cl}) \mathrm{C}(\mathrm{Cl})[\mathrm{O}]+\mathrm{NO}_{2}\end{array}$ & $\mathrm{k}=4.05 \cdot 10^{-12} \exp (360 / \mathrm{T})$ & implemented from MCM \\
\hline G69凶 & $\begin{array}{l}\mathrm{OC}(\mathrm{Cl})(\mathrm{Cl}) \mathrm{C}(\mathrm{Cl}) \mathrm{O}[\mathrm{O}]+\mathrm{NO}_{3} \rightarrow \mathrm{OC}(\mathrm{Cl})(\mathrm{Cl}) \mathrm{C}(\mathrm{Cl})[\mathrm{O}]+ \\
\mathrm{NO}_{2}\end{array}$ & $\mathrm{k}=2.30 \cdot 10^{-12}$ & \\
\hline G70(2) & $\begin{array}{l}\mathrm{OC}(\mathrm{Cl})(\mathrm{Cl}) \mathrm{C}(\mathrm{Cl}) \mathrm{O}[\mathrm{O}] \rightarrow \\
0.2 \mathrm{OC}(\mathrm{Cl})(\mathrm{Cl}) \mathrm{C}(\mathrm{O}) \mathrm{Cl}+0.6 \mathrm{OC}(\mathrm{Cl})(\mathrm{Cl}) \mathrm{C}(\mathrm{Cl})[\mathrm{O}] \\
+0.2 \mathrm{OC}(\mathrm{Cl})(\mathrm{Cl}) \mathrm{C}(=\mathrm{O}) \mathrm{Cl}\end{array}$ & $\mathrm{k}=8.80 \cdot 10^{-13} * \mathrm{RO}_{2}$ & implemented from MCM \\
\hline G71区 & $\mathrm{OC}(\mathrm{Cl}) \mathrm{C}(\mathrm{Cl}) \mathrm{O}[\mathrm{O}]+\mathrm{HO}_{2} \rightarrow \mathrm{OC}(\mathrm{Cl}) \mathrm{C}(\mathrm{Cl}) \mathrm{OO}$ & $\mathrm{k}=3.30 \cdot 10^{-13} \exp (820 / \mathrm{T})$ & \\
\hline G72区 & $\mathrm{OC}(\mathrm{Cl}) \mathrm{C}(\mathrm{Cl}) \mathrm{O}[\mathrm{O}]+\mathrm{NO} \rightarrow \mathrm{OC}(\mathrm{Cl}) \mathrm{C}(\mathrm{Cl})[\mathrm{O}]+\mathrm{NO}_{2}$ & $\mathrm{k}=4.05 \cdot 10^{-12} \exp (360 / \mathrm{T})$ & \\
\hline G73凶 & $\mathrm{OC}(\mathrm{Cl}) \mathrm{C}(\mathrm{Cl}) \mathrm{O}[\mathrm{O}]+\mathrm{NO}_{3} \rightarrow \mathrm{OC}(\mathrm{Cl}) \mathrm{C}(\mathrm{Cl})[\mathrm{O}]+\mathrm{NO}_{2}$ & $\mathrm{k}=2.30 \cdot 10^{-12}$ & \\
\hline
\end{tabular}




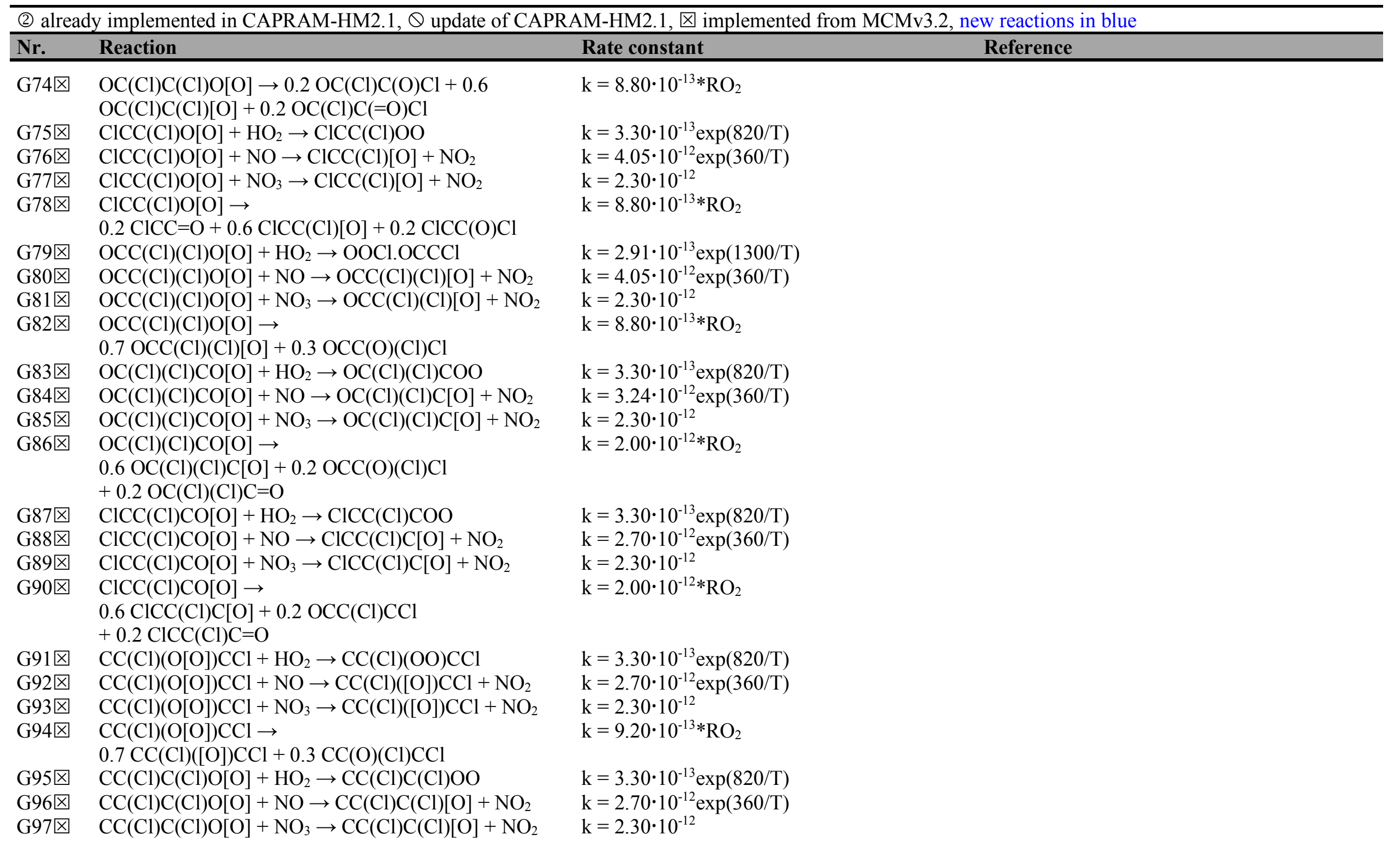




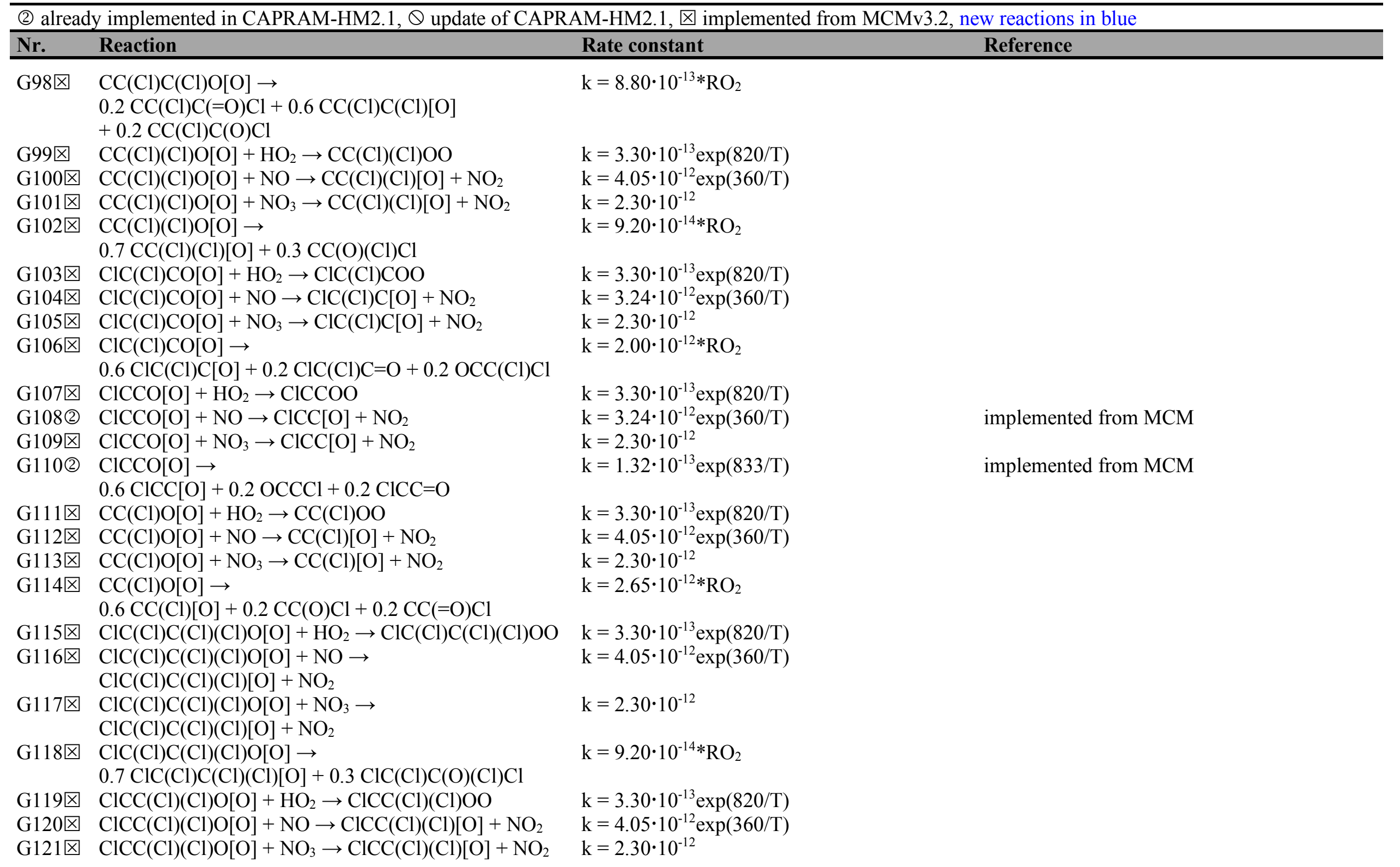




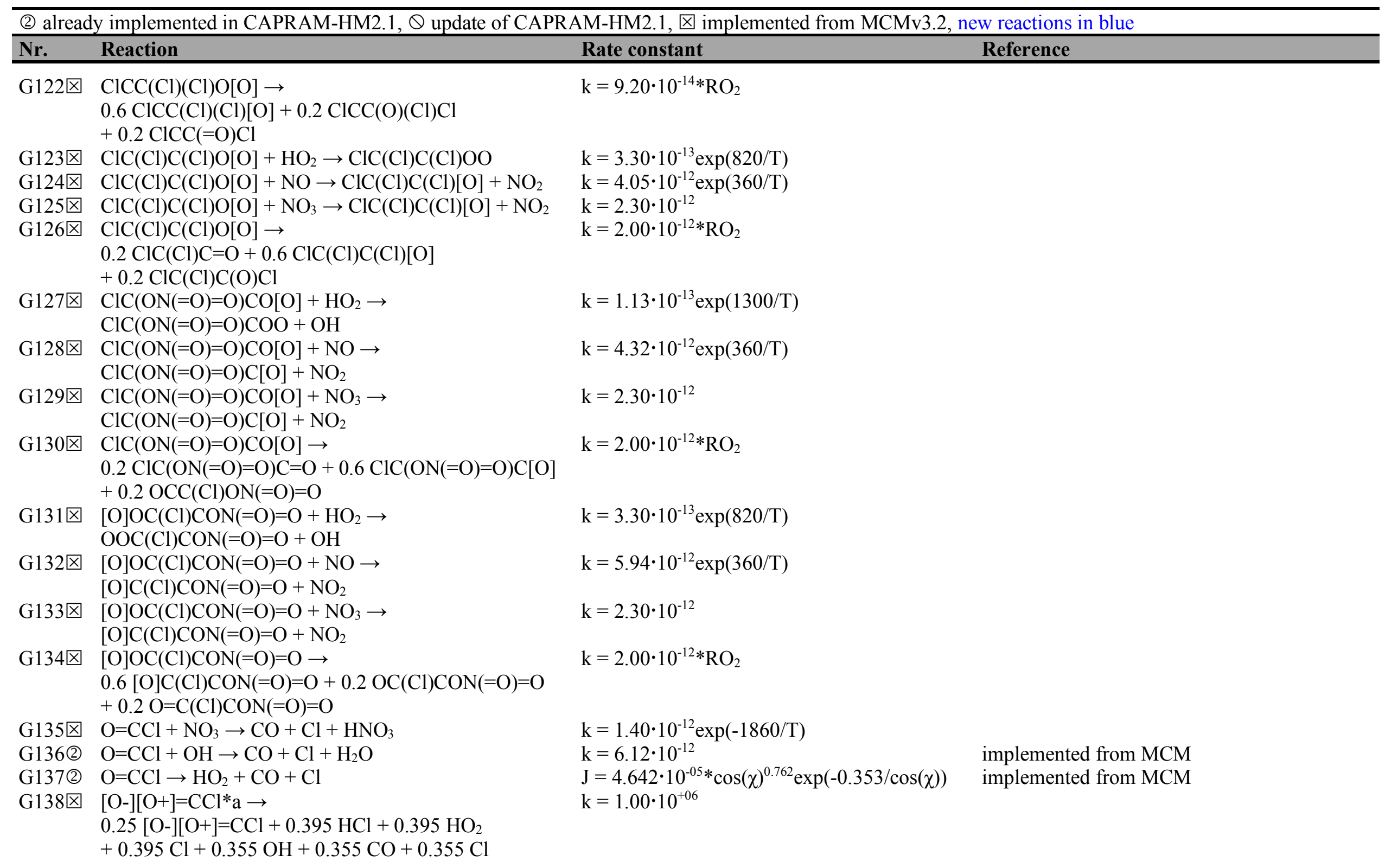




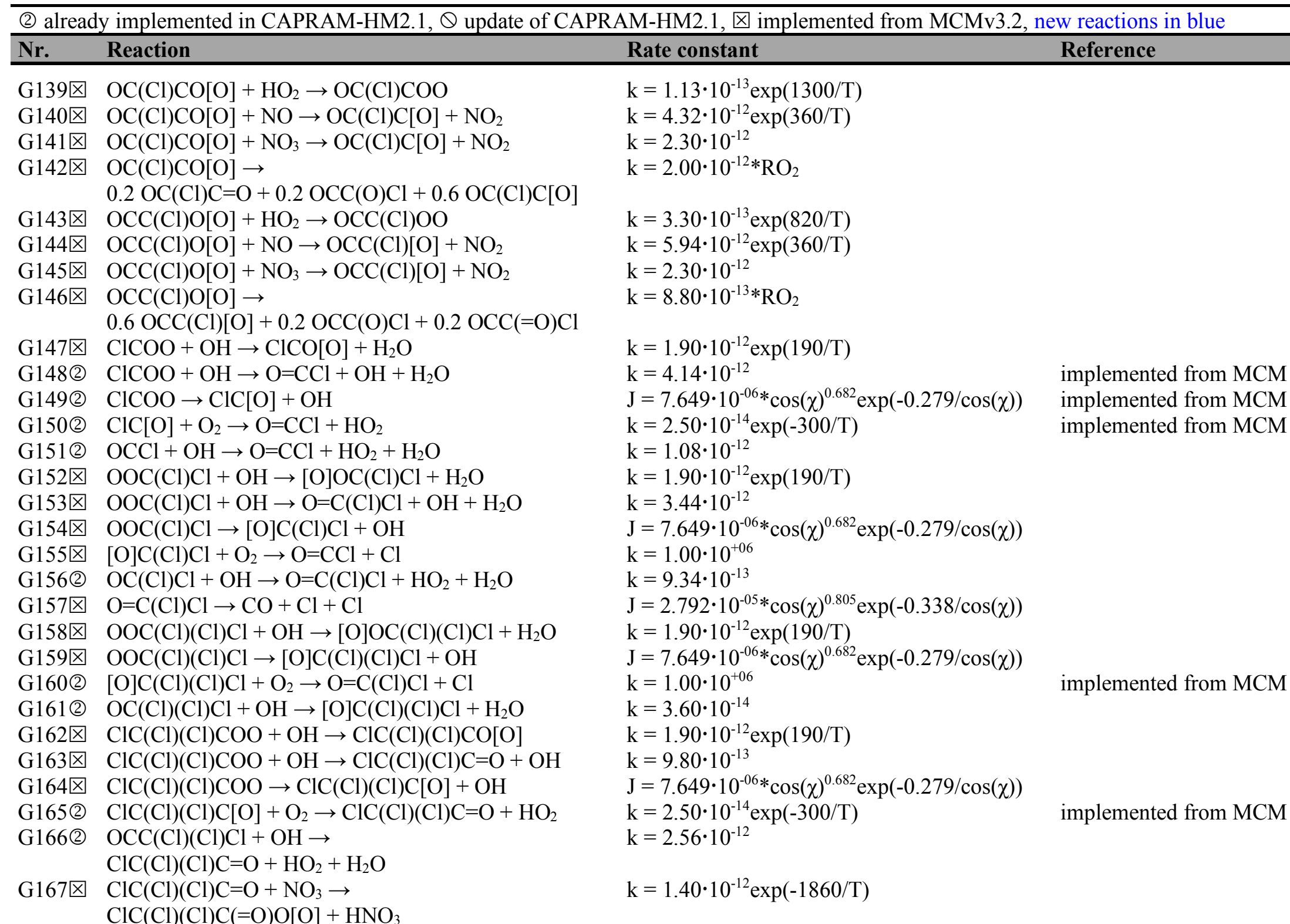




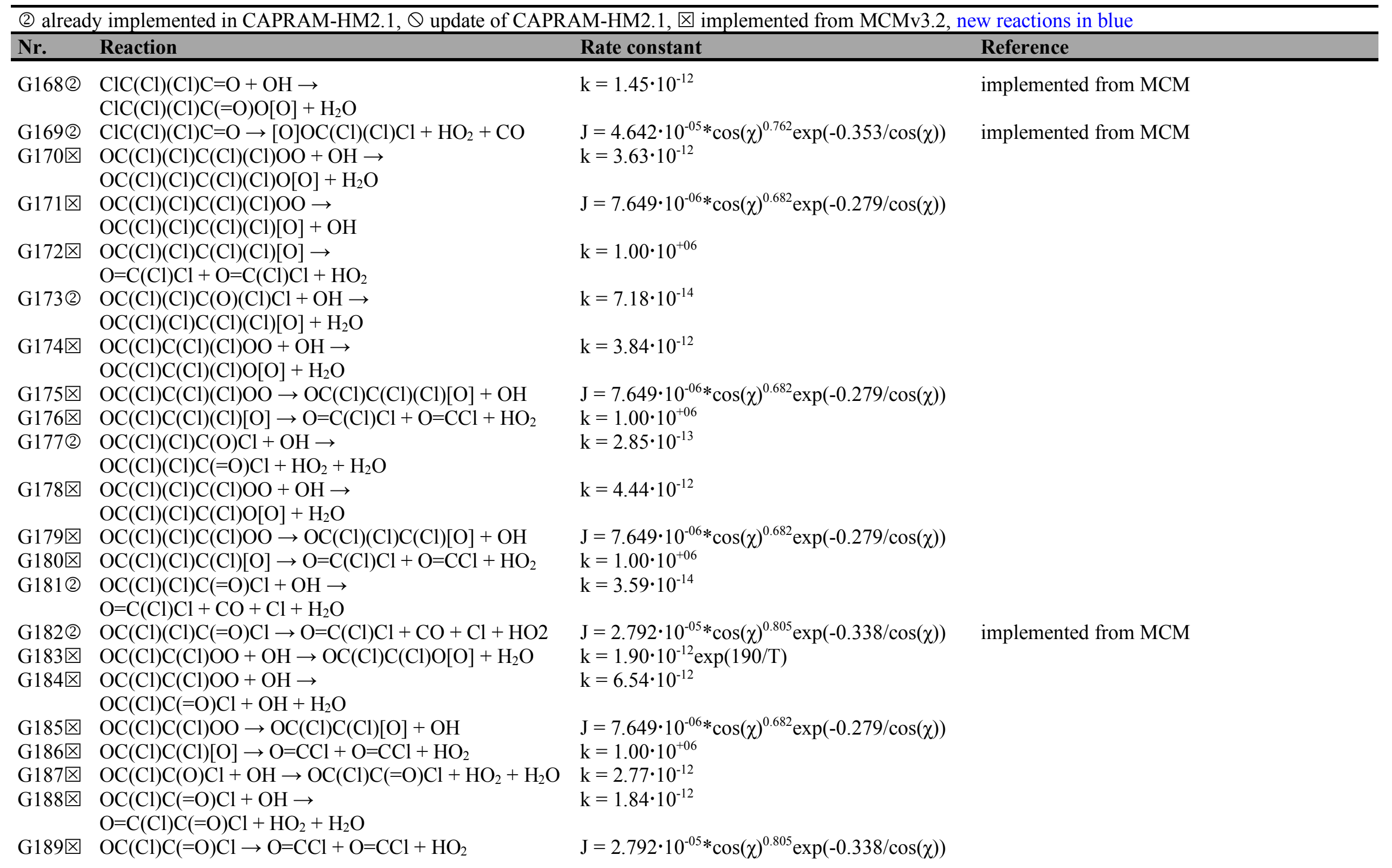




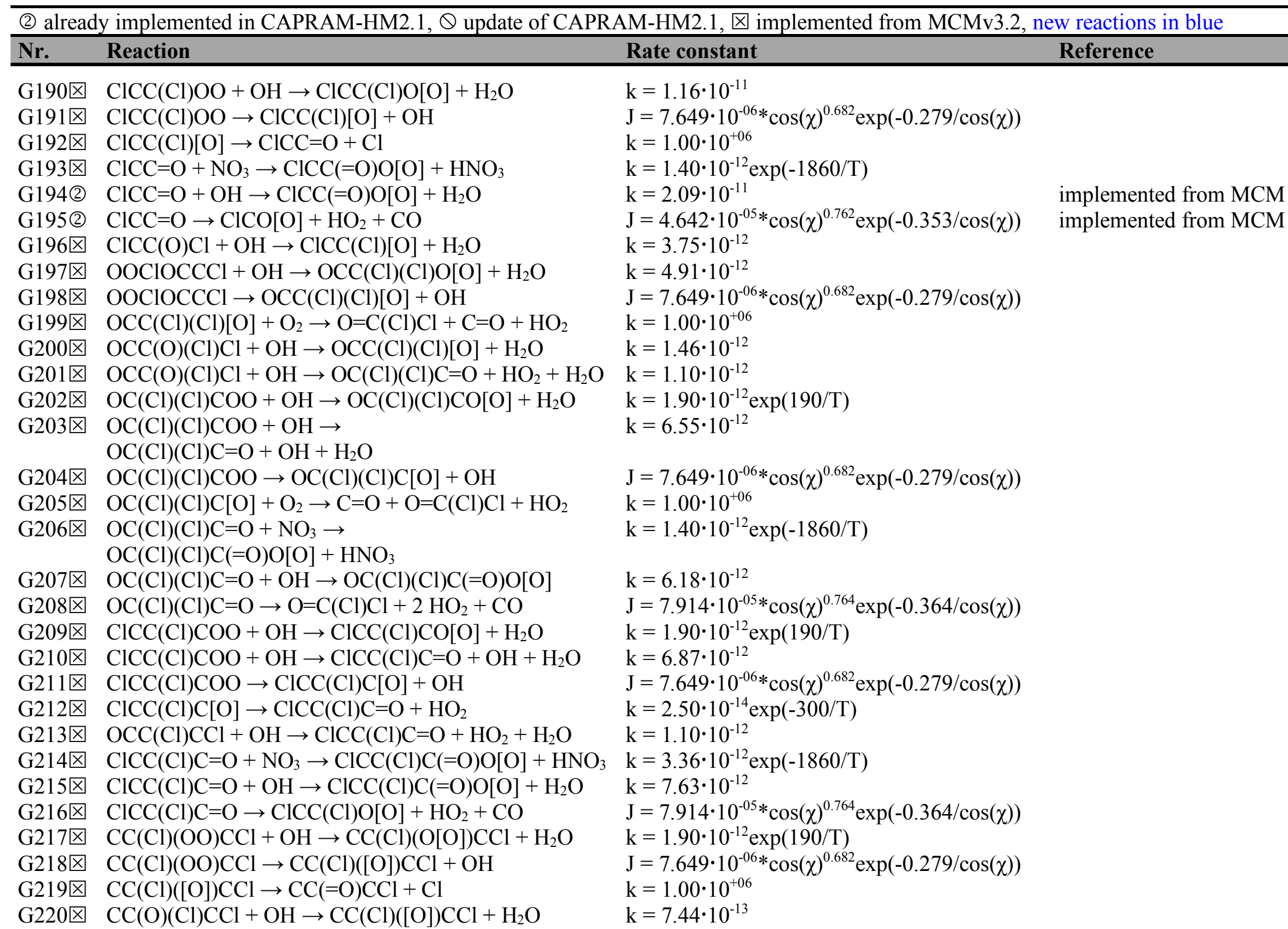




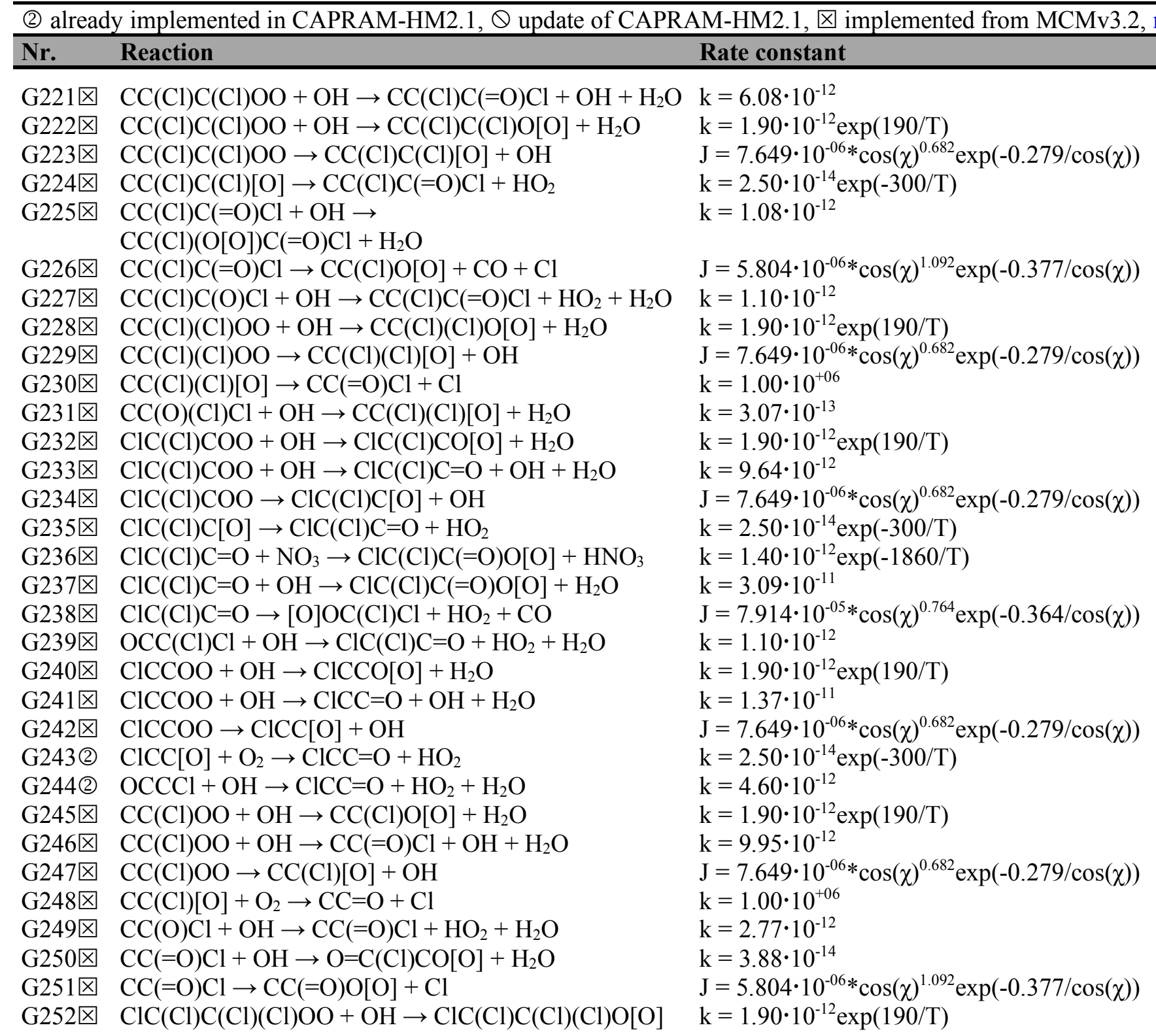

ns in blue

Reference

implemented from MCM 


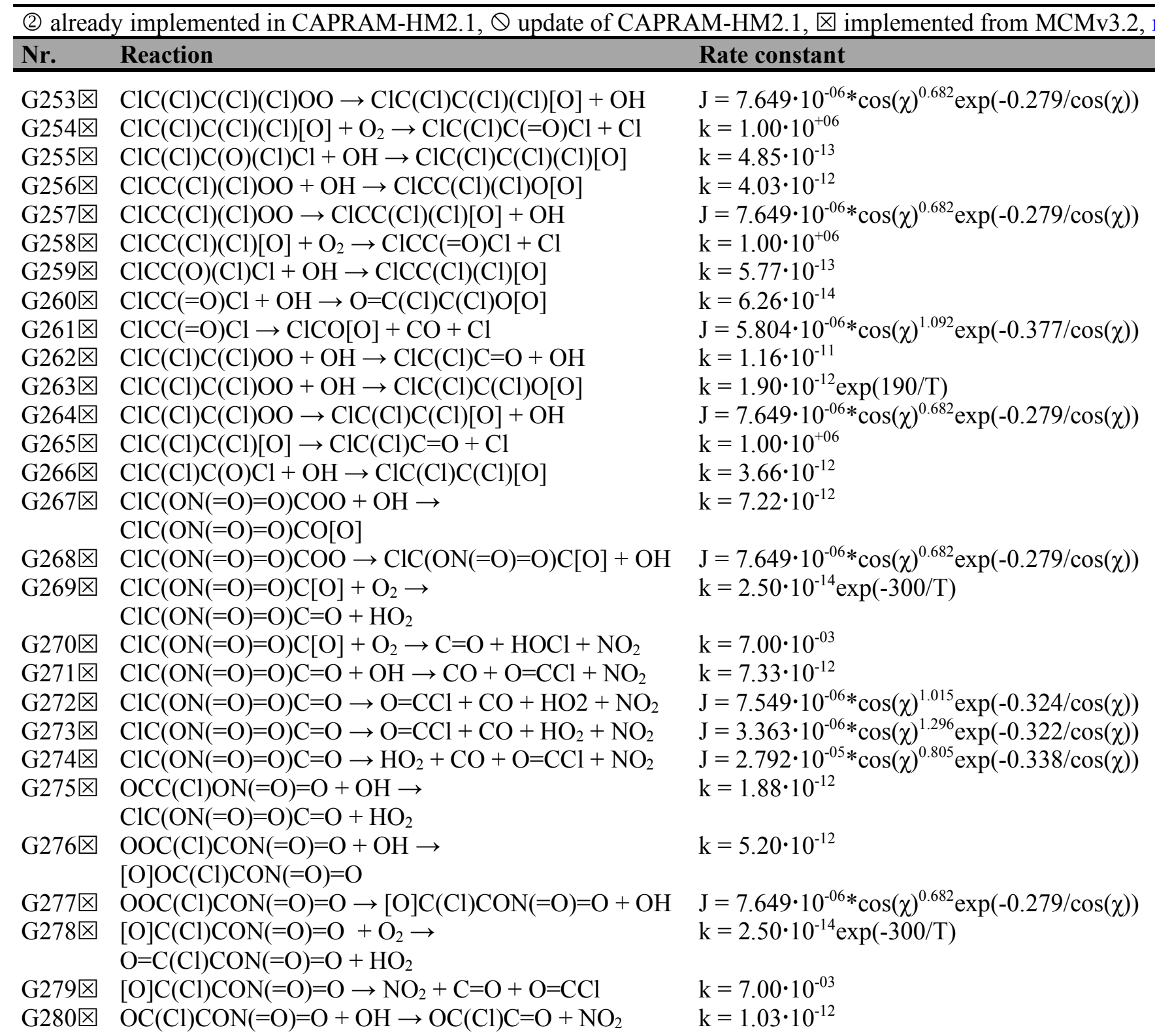

in blue Reference 


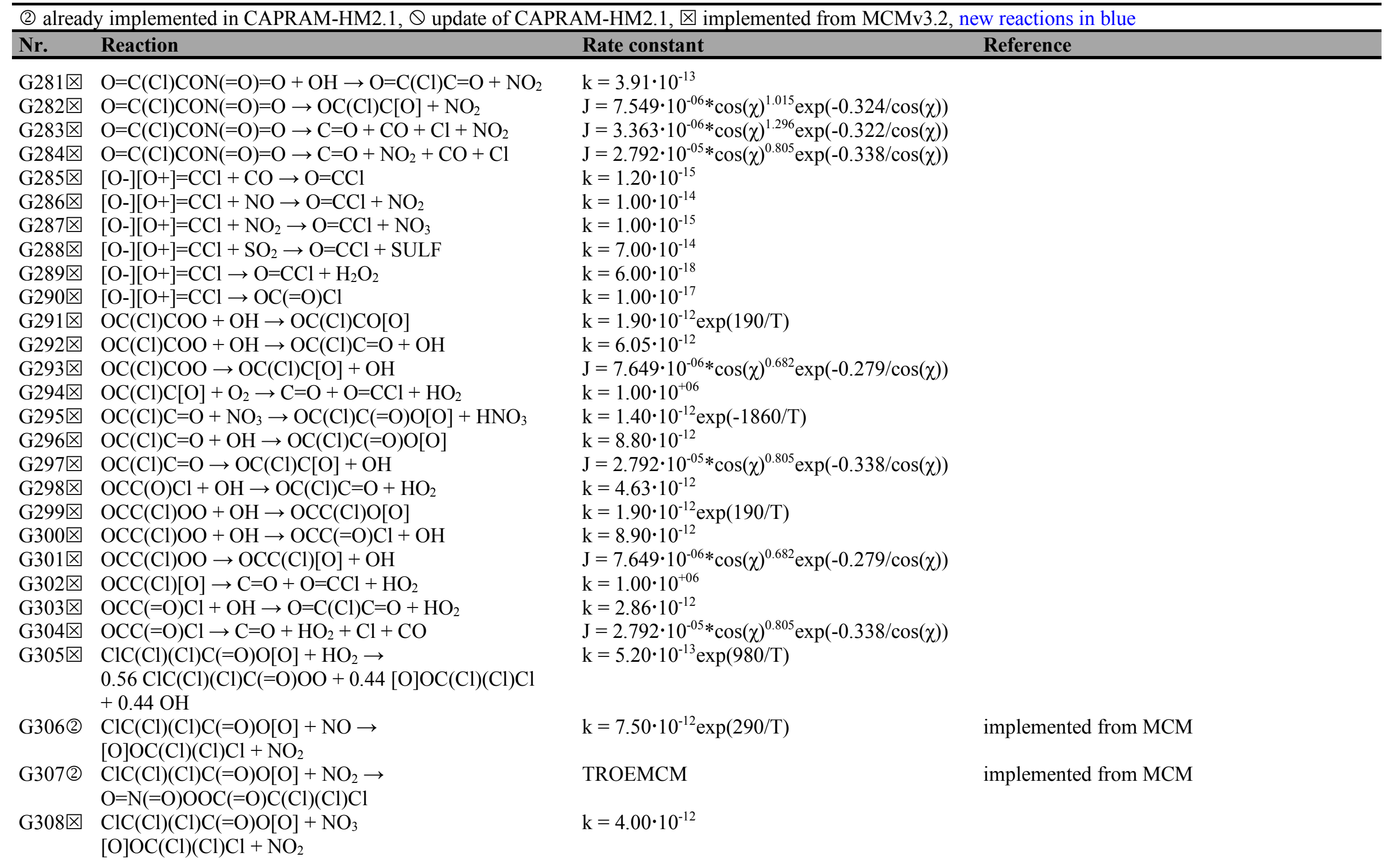




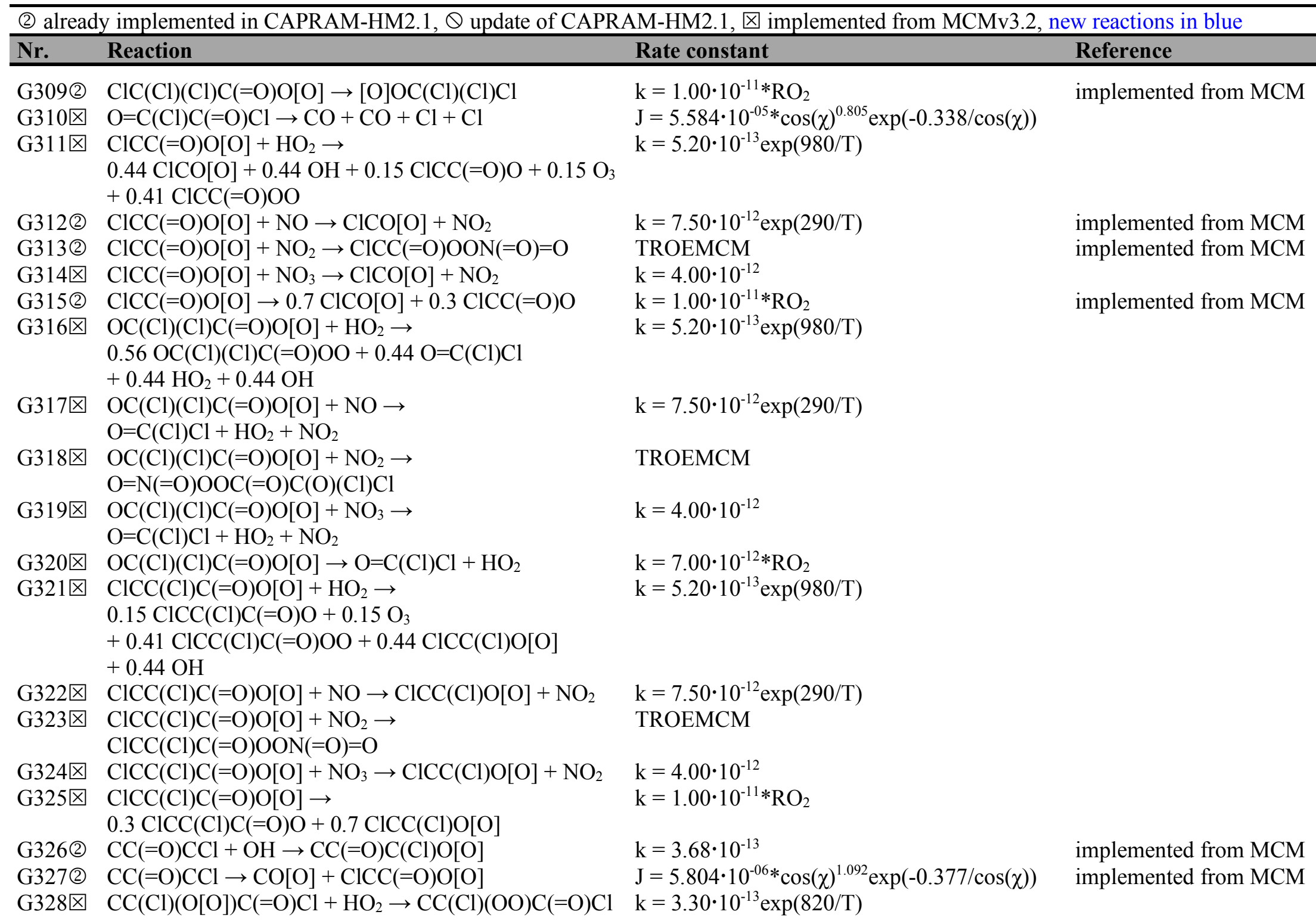




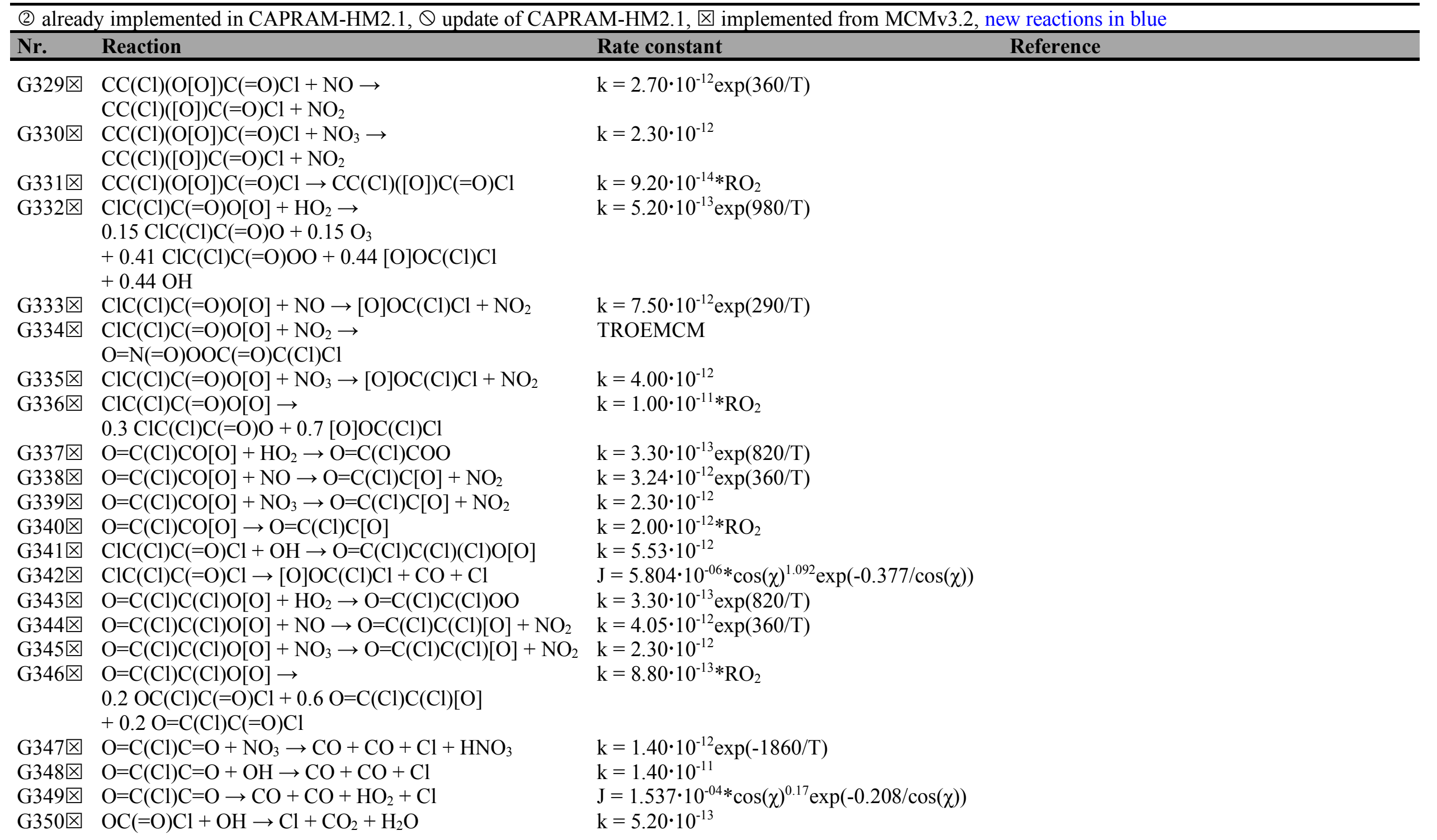




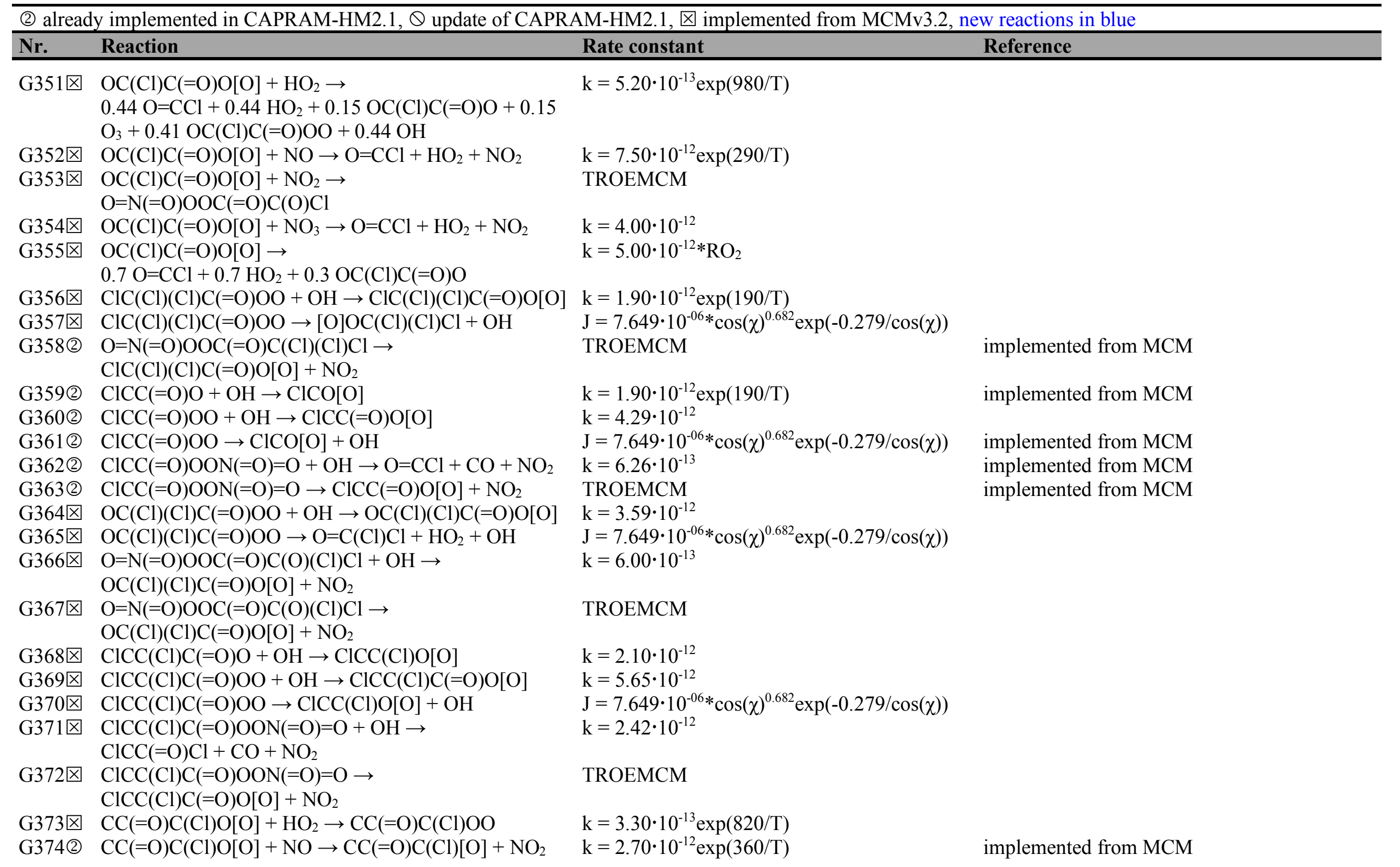




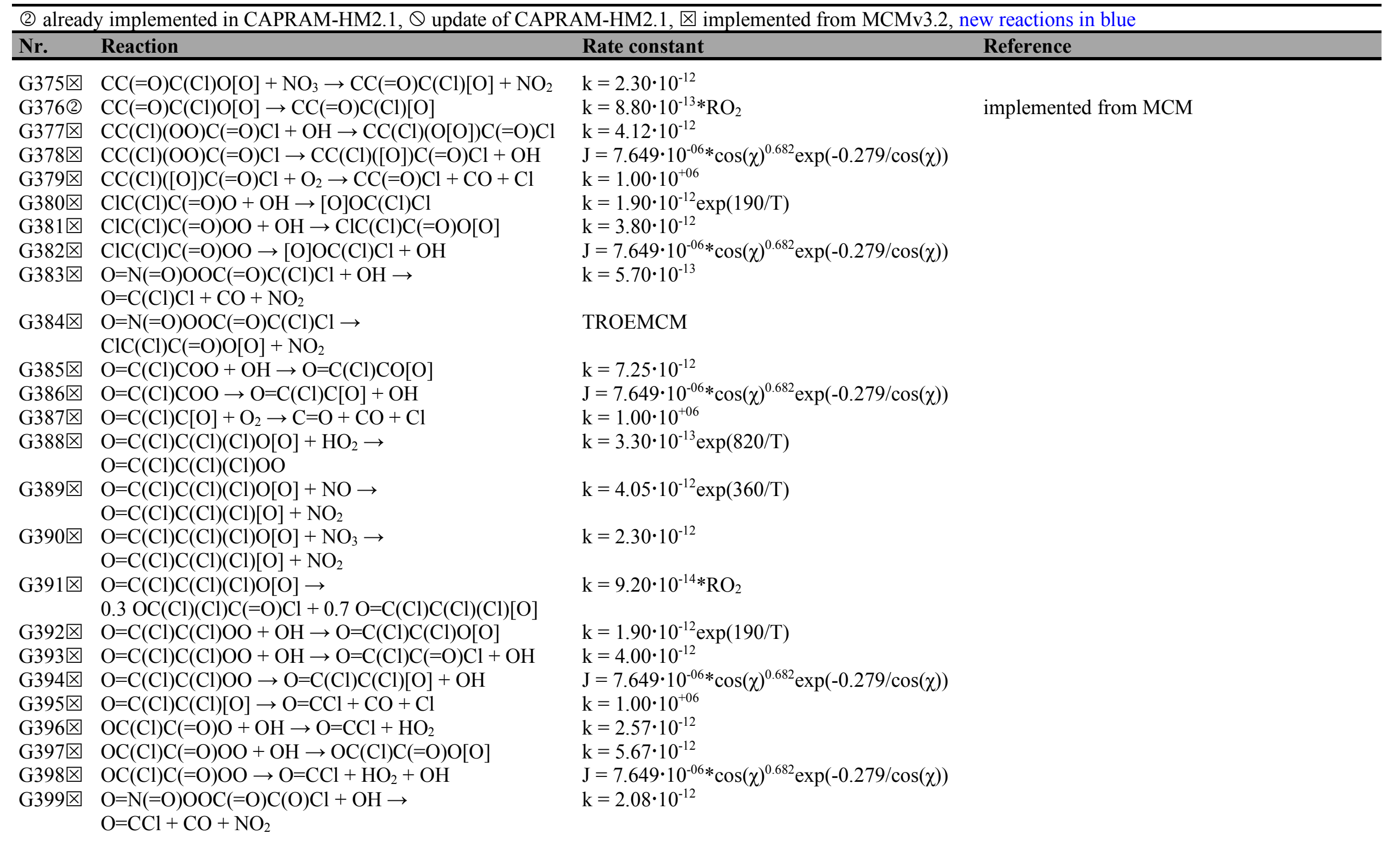




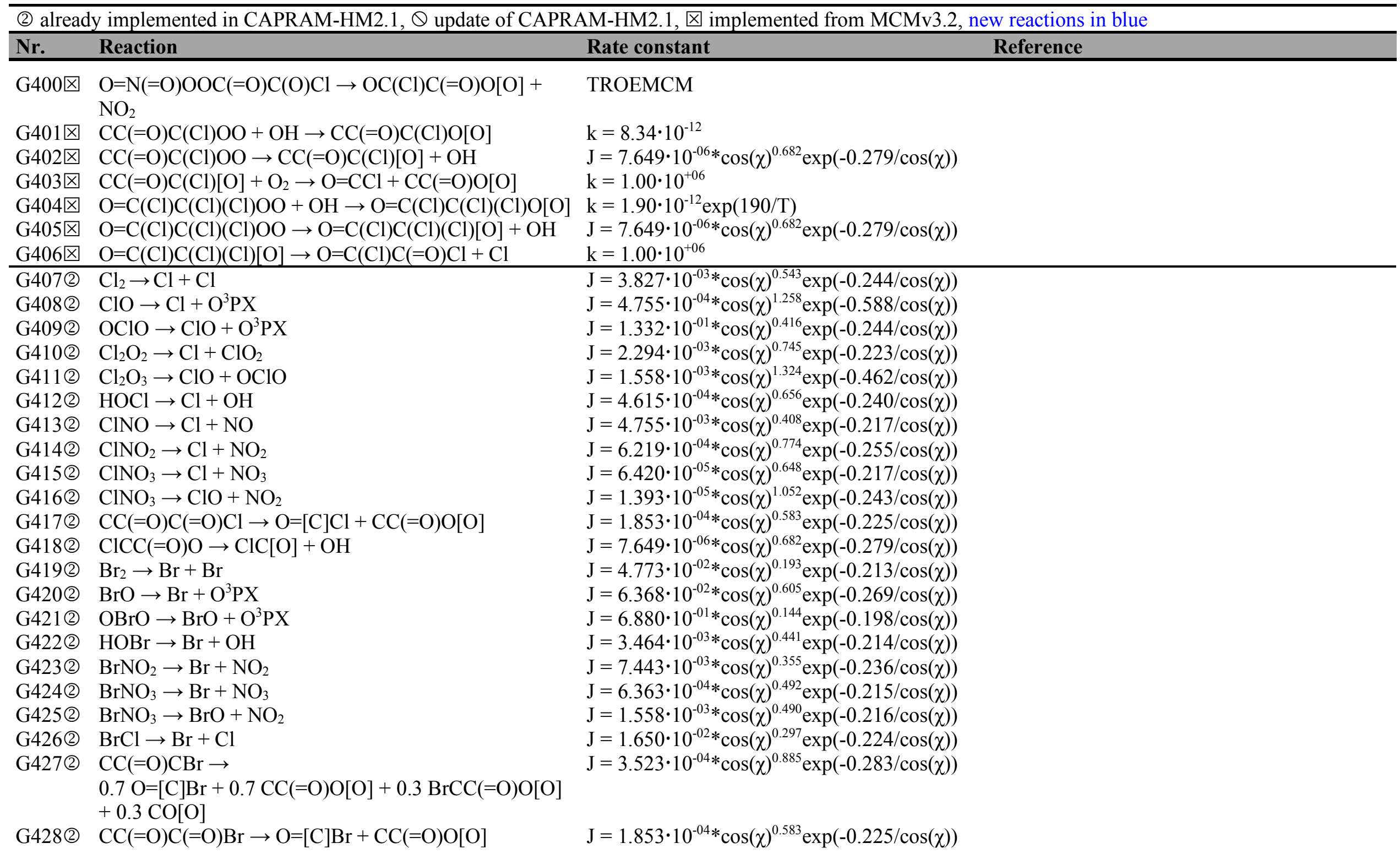




\begin{tabular}{|c|c|c|c|}
\hline Nr. & Reaction & Rate constant & Reference \\
\hline G429(2) & $\mathrm{BrC}(\mathrm{Br}) \mathrm{Br} \rightarrow \mathrm{Br}+\mathrm{BrC}(\mathrm{Br}) \mathrm{O}[\mathrm{O}]$ & $\mathrm{J}=2.228 \cdot 10^{-06 *} \cos (\chi)^{1.471} \exp (-0.230 / \cos (\chi))$ & \\
\hline $\mathrm{G} 430$ (2) & $\mathrm{BrCBr} \rightarrow \mathrm{Br}+\mathrm{BrCO}[\mathrm{O}]$ & $\mathrm{J}=5.600 \cdot 10^{-09} * \cos (\chi)^{2.763} \exp (-1.922 / \cos (\chi))$ & \\
\hline G431(2) & $\mathrm{O}=\mathrm{C}(\mathrm{Br}) \mathrm{Br} \rightarrow 2.0 \mathrm{Br}+\mathrm{CO}$ & $\mathrm{J}=4.377 \cdot 10^{-06 *} \cos (\chi)^{1.360} \exp (-0.273 / \cos (\chi))$ & \\
\hline G432(2) & $\mathrm{I}_{2} \rightarrow \mathrm{I}+\mathrm{I}$ & $\mathrm{J}=2.165 \cdot 10^{-01} * \cos (\chi)^{0.125} \exp (-0.185 / \cos (\chi))$ & \\
\hline G433(2) & $\mathrm{IO} \rightarrow \mathrm{I}+\mathrm{O}^{3} \mathrm{PX}$ & $\mathrm{J}=2.640 \cdot 10^{-03 *} \cos (\chi)^{0.240} \exp (-0.240 / \cos (\chi))$ & \\
\hline G434(2) & $\mathrm{OIO} \rightarrow \mathrm{I}$ & $\mathrm{J}=4.054 \cdot 10^{-02 *} \cos (\chi)^{0.119} \exp (-0.185 / \cos (\chi))$ & \\
\hline G435(2) & $\mathrm{OIO} \rightarrow \mathrm{IO}+\mathrm{O}^{3} \mathrm{PX}$ & $\mathrm{J}=1.894 \cdot 10^{-03 *} \cos (\chi)^{0.119} \exp (-0.185 / \cos (\chi))$ & \\
\hline G436(2) & $\mathrm{I}_{2} \mathrm{O}_{2} \rightarrow 2.0 \mathrm{I}$ & $\mathrm{J}=2.294 \cdot 10^{-03 *} \cos (\chi)^{0.745} \exp (-0.223 / \cos (\chi))$ & \\
\hline $\mathrm{G} 437(2)$ & $\mathrm{HI} \rightarrow \mathrm{I}+\mathrm{HO}_{2}$ & $\mathrm{~J}=2.104 \cdot 10^{-04 *} \cos (\chi)^{1.123} \exp (-0.281 / \cos (\chi))$ & \\
\hline G438(2) & $\mathrm{HOI} \rightarrow \mathrm{I}+\mathrm{OH}$ & $\mathrm{J}=1.469 \cdot 10^{-02} * \cos (\chi)^{0.342} \exp (-0.236 / \cos (\chi))$ & \\
\hline G439(2) & $\mathrm{INO} \rightarrow \mathrm{I}+\mathrm{NO}$ & $\mathrm{J}=4.849 \cdot 10^{-02 *} \cos (\chi)^{0.284} \exp (-0.232 / \cos (\chi))$ & \\
\hline G440(2) & $\mathrm{INO}_{2} \rightarrow \mathrm{I}+\mathrm{NO}_{2}$ & $\mathrm{~J}=5.036 \cdot 10^{-03 *} \cos (\chi)^{0.568} \exp (-0.256 / \cos (\chi))$ & \\
\hline G441(2) & $\mathrm{INO}_{3} \rightarrow \mathrm{I}+\mathrm{NO}_{3}$ & $\mathrm{~J}=6.599 \cdot 10^{-02 *} \cos (\chi)^{0.530} \exp (-0.243 / \cos (\chi))$ & \\
\hline G442(2) & $\mathrm{INO}_{3} \rightarrow \mathrm{IO}+\mathrm{NO}_{2}$ & $\mathrm{~J}=1.165 \cdot 10^{-02 *} \cos (\chi)^{0.528} \exp (-0.244 / \cos (\chi))$ & \\
\hline $\mathrm{G} 443(2)$ & $\mathrm{ICl} \rightarrow \mathrm{I}+\mathrm{Cl}$ & $\mathrm{J}=3.403 \cdot 10^{-02 *} \cos (\chi)^{0.179} \exp (-0.207 / \cos (\chi))$ & \\
\hline G444(2) & $\mathrm{IBr} \rightarrow \mathrm{I}+\mathrm{Br}$ & $\mathrm{J}=1.000 \cdot 10^{-01 *} \cos (\chi)^{0.149} \exp (-0.197 / \cos (\chi))$ & \\
\hline G445(2) & $\mathrm{CC}(\mathrm{I}) \mathrm{C} \rightarrow \mathrm{I}+\mathrm{CC}(\mathrm{O}[\mathrm{O}]) \mathrm{C}$ & $\mathrm{J}=3.731 \cdot 10^{-05 *} \cos (\chi)^{1.292} \exp (-0.217 / \cos (\chi))$ & \\
\hline G446(2) & $\mathrm{ICC} \rightarrow \mathrm{I}+\mathrm{CCO}[\mathrm{O}]$ & $\mathrm{J}=1.386 \cdot 10^{-05 *} \cos (\chi)^{1.324} \exp (-0.224 / \cos (\chi))$ & \\
\hline G447(2) & $\mathrm{ICC}=\mathrm{O} \rightarrow[\mathrm{O}] \mathrm{OCI}+\mathrm{CO}+\mathrm{HO}_{2}$ & $\mathrm{~J}=4.642 \cdot 10^{-05 *} \cos (\chi)^{0.762} \exp (-0.353 / \cos (\chi))$ & \\
\hline G448(2) & $\mathrm{ICC}(=\mathrm{O}) \mathrm{OO} \rightarrow[\mathrm{O}] \mathrm{OCI}+\mathrm{CO}_{2}+\mathrm{OH}$ & $\mathrm{J}=7.649 \cdot 10^{-06 *} \cos (\chi)^{0.682} \exp (-0.279 / \cos (\chi))$ & \\
\hline G449(2) & $\mathrm{ICI} \rightarrow \mathrm{I}+[\mathrm{O}] \mathrm{OCI}$ & $\mathrm{J}=1.496 \cdot 10^{-02 *} \cos (\chi)^{0.801} \exp (-0.265 / \cos (\chi))$ & \\
\hline G450(2) & $\mathrm{CI} \rightarrow \mathrm{I}+\mathrm{CO}[\mathrm{O}]$ & $\mathrm{J}=1.206 \cdot 10^{-05 *} \cos (\chi)^{1.254} \exp (-0.231 / \cos (\chi))$ & \\
\hline G451(2) & $\mathrm{OOCI} \rightarrow[\mathrm{O}] \mathrm{CI}+\mathrm{OH}$ & $\mathrm{J}=7.649 \cdot 10^{-06 *} \cos (\chi)^{0.682} \exp (-0.279 / \cos (\chi))$ & \\
\hline G452(2) & $\mathrm{O}=\mathrm{CI} \rightarrow \mathrm{I}+\mathrm{CO}+\mathrm{HO}_{2}$ & $\mathrm{~J}=2.547 \cdot 10^{-05 *} \cos (\chi)^{1.393} \exp (-0.361 / \cos (\chi))$ & \\
\hline G453(2) & $\mathrm{ClCI} \rightarrow \mathrm{I}+\mathrm{ClCO}[\mathrm{O}]$ & $\mathrm{J}=6.910 \cdot 10^{-04 *} \cos (\chi)^{1.057} \exp (-0.238 / \cos (\chi))$ & \\
\hline G454(2) & $\mathrm{BrCI} \rightarrow \mathrm{I}+\mathrm{BrCO}[\mathrm{O}]$ & $\mathrm{J}=4.261 \cdot 10^{-04 *} \cos (\chi)^{0.976} \exp (-0.250 / \cos (\chi))$ & \\
\hline G455(2) & $\mathrm{Cl}+\mathrm{O}_{3} \rightarrow \mathrm{ClO}$ & $\mathrm{k}=2.80 \cdot 10^{-11} \exp (-250 / \mathrm{T})$ & \\
\hline G456(2) & $\mathrm{Cl}+\mathrm{H}_{2} \rightarrow \mathrm{HCl}+\mathrm{HO}_{2}$ & $\mathrm{k}=3.90 \cdot 10^{-11} \exp (-2310 / \mathrm{T})$ & \\
\hline G457Q & $\mathrm{Cl}+\mathrm{HO}_{2} \rightarrow \mathrm{HCl}$ & $\mathrm{k}=3.48 \cdot 10^{-11}$ & revised after IUPAC \\
\hline G458Q & $\mathrm{Cl}+\mathrm{HO}_{2} \rightarrow \mathrm{ClO}+\mathrm{OH}$ & $\mathrm{k}=7.48 \cdot 10^{-11} \exp (-620 / \mathrm{T})$ & revised after IUPAC \\
\hline G459(2) & $\mathrm{Cl}+\mathrm{H}_{2} \mathrm{O}_{2} \rightarrow \mathrm{HCl}+\mathrm{HO}_{2}$ & $\mathrm{k}=1.10 \cdot 10^{-11} \exp (-980 / \mathrm{T})$ & \\
\hline G460(2) & $\mathrm{Cl}_{2}+\mathrm{OH} \rightarrow \mathrm{HOCl}+\mathrm{Cl}$ & $\mathrm{k}=3.60 \cdot 10^{-12} \exp (-1200 / \mathrm{T})$ & \\
\hline
\end{tabular}




\begin{tabular}{|c|c|c|c|}
\hline Nr. & Reaction & Rate constant & Reference \\
\hline G461 (2) & $\mathrm{ClO}+\mathrm{O}_{3} \rightarrow \mathrm{ClO}_{2}$ & $\mathrm{k}=2.00 \cdot 10^{-12} \exp (-3600 / \mathrm{T})$ & \\
\hline G462(2) & $\mathrm{ClO}+\mathrm{O}_{3} \rightarrow \mathrm{OClO}$ & $\mathrm{k}=1.00 \cdot 10^{-12} \exp (-4000 / \mathrm{T})$ & \\
\hline G463(2) & $\mathrm{ClO}+\mathrm{OH} \rightarrow 0.94 \mathrm{HO}_{2}+0.94 \mathrm{Cl}+0.06 \mathrm{HCl}$ & $\mathrm{k}=7.30 \cdot 10^{-12} \exp (300 / \mathrm{T})$ & \\
\hline G464(2) & $\mathrm{ClO}+\mathrm{HO}_{2} \rightarrow \mathrm{HOCl}$ & $\mathrm{k}=2.20 \cdot 10^{-12} \exp (340 / \mathrm{T})$ & \\
\hline G465(2) & $\mathrm{ClO}+\mathrm{ClO} \rightarrow \mathrm{Cl}_{2}$ & $\mathrm{k}=1.00 \cdot 10^{-12} \exp (-1590 / \mathrm{T})$ & \\
\hline G466(2) & $\mathrm{ClO}+\mathrm{ClO} \rightarrow \mathrm{Cl}+\mathrm{ClO}_{2}$ & $\mathrm{k}=3.00 \cdot 10^{-11} \exp (-2450 / \mathrm{T})$ & \\
\hline G467(2) & $\mathrm{ClO}+\mathrm{ClO} \rightarrow \mathrm{Cl}+\mathrm{OClO}$ & $\mathrm{k}=3.50 \cdot 10^{-13} \exp (-1370 / \mathrm{T})$ & \\
\hline G468@ & $\mathrm{ClO}+\mathrm{ClO} \rightarrow \mathrm{Cl}_{2} \mathrm{O}_{2}$ & TROE & Burkholder et al. ${ }^{43}$ \\
\hline G469(2) & $\mathrm{Cl}+\mathrm{O}_{2} \rightarrow \mathrm{ClO}_{2}$ & TROE & \\
\hline G470(2) & $\mathrm{ClO}_{2} \rightarrow \mathrm{Cl}$ & $\mathrm{k}=2.80 \cdot 10^{-10} \exp (-1820 / \mathrm{T})$ & \\
\hline G471(2) & $\mathrm{Cl}+\mathrm{ClO}_{2} \rightarrow 0.1 \mathrm{ClO}+0.95 \mathrm{Cl}_{2}$ & $\mathrm{k}=2.42 \cdot 10^{-10}$ & \\
\hline G472(2) & $\mathrm{Cl}_{2} \mathrm{O}_{2} \rightarrow \mathrm{ClO}+\mathrm{ClO}$ & TROEXP & \\
\hline G473(2) & $\mathrm{Cl}_{2} \mathrm{O}_{2}+\mathrm{O}_{3} \rightarrow \mathrm{ClO}+\mathrm{ClO}_{2}$ & $\mathrm{k}=1.00 \cdot 10^{-19}$ & \\
\hline G474(2) & $\mathrm{Cl}_{2} \mathrm{O}_{2}+\mathrm{Cl} \rightarrow \mathrm{Cl}_{2}+\mathrm{ClO}_{2}$ & $\mathrm{k}=7.60 \cdot 10^{-11} \exp (65 / \mathrm{T})$ & \\
\hline G475(2) & $\mathrm{OClO}+\mathrm{OH} \rightarrow \mathrm{HOCl}$ & $\mathrm{k}=1.40 \cdot 10^{-12} \exp (600 / \mathrm{T})$ & \\
\hline G476(2) & $\mathrm{OClO}+\mathrm{Cl} \rightarrow \mathrm{ClO}+\mathrm{ClO}$ & $\mathrm{k}=3.20 \cdot 10^{-11} \exp (170 / \mathrm{T})$ & \\
\hline G477(2) & $\mathrm{OClO}+\mathrm{ClO} \rightarrow \mathrm{Cl}_{2} \mathrm{O}_{3}$ & TROE & \\
\hline G478(2) & $\mathrm{Cl}_{2} \mathrm{O}_{3} \rightarrow \mathrm{OClO}+\mathrm{ClO}$ & TROEXP & \\
\hline G479(2) & $\mathrm{HCl}+\mathrm{OH} \rightarrow \mathrm{Cl}$ & $\mathrm{k}=1.70 \cdot 10^{-12} \exp (-230 / \mathrm{T})$ & \\
\hline G480(2) & $\mathrm{HOCl}+\mathrm{OH} \rightarrow \mathrm{ClO}$ & $\mathrm{k}=3.00 \cdot 10^{-12} \exp (-500 / \mathrm{T})$ & \\
\hline \multirow[t]{2}{*}{ G481@ } & $\mathrm{HOCl}+\mathrm{Cl} \rightarrow$ & $\mathrm{k}=3.40 \cdot 10^{-12} \exp (-130 / \mathrm{T})$ & Burkholder et al. ${ }^{43}$ \\
\hline & $0.76 \mathrm{HCl}+0.76 \mathrm{ClO}+0.24 \mathrm{Cl}_{2}+0.24 \mathrm{OH}$ & & \\
\hline G482(2) & $\mathrm{ClO}+\mathrm{NO} \rightarrow \mathrm{Cl}+\mathrm{NO}_{2}$ & $\mathrm{k}=6.20 \cdot 10^{-12} \exp (295 / \mathrm{T})$ & \\
\hline G483(2) & $\mathrm{OClO}+\mathrm{NO} \rightarrow \mathrm{ClO}+\mathrm{NO}_{2}$ & $\mathrm{k}=1.10 \cdot 10^{-13} \exp (350 / \mathrm{T})$ & \\
\hline G484(2) & $\mathrm{Cl}+\mathrm{NO}_{3} \rightarrow \mathrm{ClO}+\mathrm{NO}_{2}$ & $\mathrm{k}=2.40 \cdot 10^{-11}$ & \\
\hline G485(2) & $\mathrm{ClO}+\mathrm{NO}_{3} \rightarrow 0.68 \mathrm{ClO}_{2}+0.32 \mathrm{OClO}+\mathrm{NO}_{2}$ & $\mathrm{k}=4.61 \cdot 10^{-13}$ & \\
\hline G486(2) & $\mathrm{Cl}+\mathrm{NO} \rightarrow \mathrm{ClNO}$ & $\mathrm{k}=[\mathrm{M}]^{*} 7.60 \cdot 10^{-32}(\mathrm{~T} / 300)^{1.8}$ & \\
\hline G487(2) & $\mathrm{Cl}+\mathrm{ClNO} \rightarrow \mathrm{Cl}_{2}+\mathrm{NO}$ & $\mathrm{k}=5.80 \cdot 10^{-11} \exp (100 / \mathrm{T})$ & \\
\hline G488(2) & $\mathrm{Cl}+\mathrm{NO}_{2} \rightarrow \mathrm{ClNO}_{2}$ & TROE & \\
\hline G489(2) & $\mathrm{ClNO}_{2}+\mathrm{OH} \rightarrow \mathrm{HOCl}+\mathrm{NO}_{2}$ & $\mathrm{k}=2.40 \cdot 10^{-12} \exp (-1250 / \mathrm{T})$ & \\
\hline G490(2) & $\mathrm{ClO}+\mathrm{NO}_{2} \rightarrow \mathrm{ClNO}_{3}$ & TROEF & \\
\hline G491(2) & $\mathrm{ClNO}_{3} \rightarrow \mathrm{ClO}+\mathrm{NO}_{2}$ & $\mathrm{k}=[\mathrm{M}]^{*} 2.75 \cdot 10^{-6} \exp (11438 / \mathrm{T})$ & \\
\hline
\end{tabular}




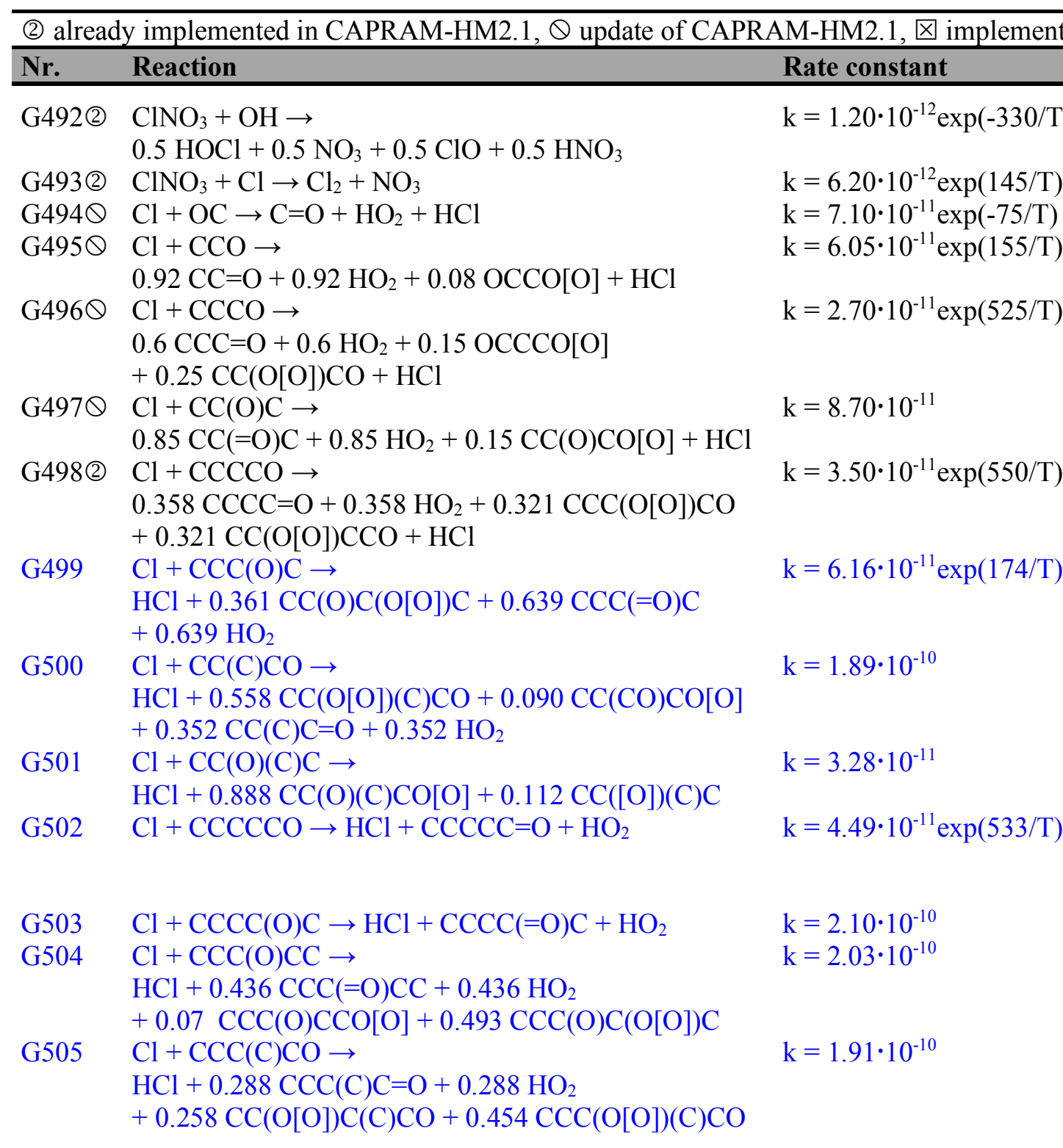

W reactions in blue Reference

revised after IUPAC revised after IUPAC

revised after IUPAC

revised after IUPAC

Ballesteros et al. ${ }^{44}$, products MCM

$\mathrm{Wu}$ et al. ${ }^{45}$, mean of two relative rate constants, products MCM

$\mathrm{Wu}$ et al. ${ }^{45}$, mean of two relative rate constants, products MCM

Rate constant mean from Lendar et al. ${ }^{46}, \mathrm{Wu}$ et al. ${ }^{45}$, Nelson et al. ${ }^{47} \&$ Garzon et al. ${ }^{48}$, product MCM

Lendar et al. ${ }^{46}$

Hurley et al. ${ }^{49}$, only measurement

Xue et al. ${ }^{50}$ 


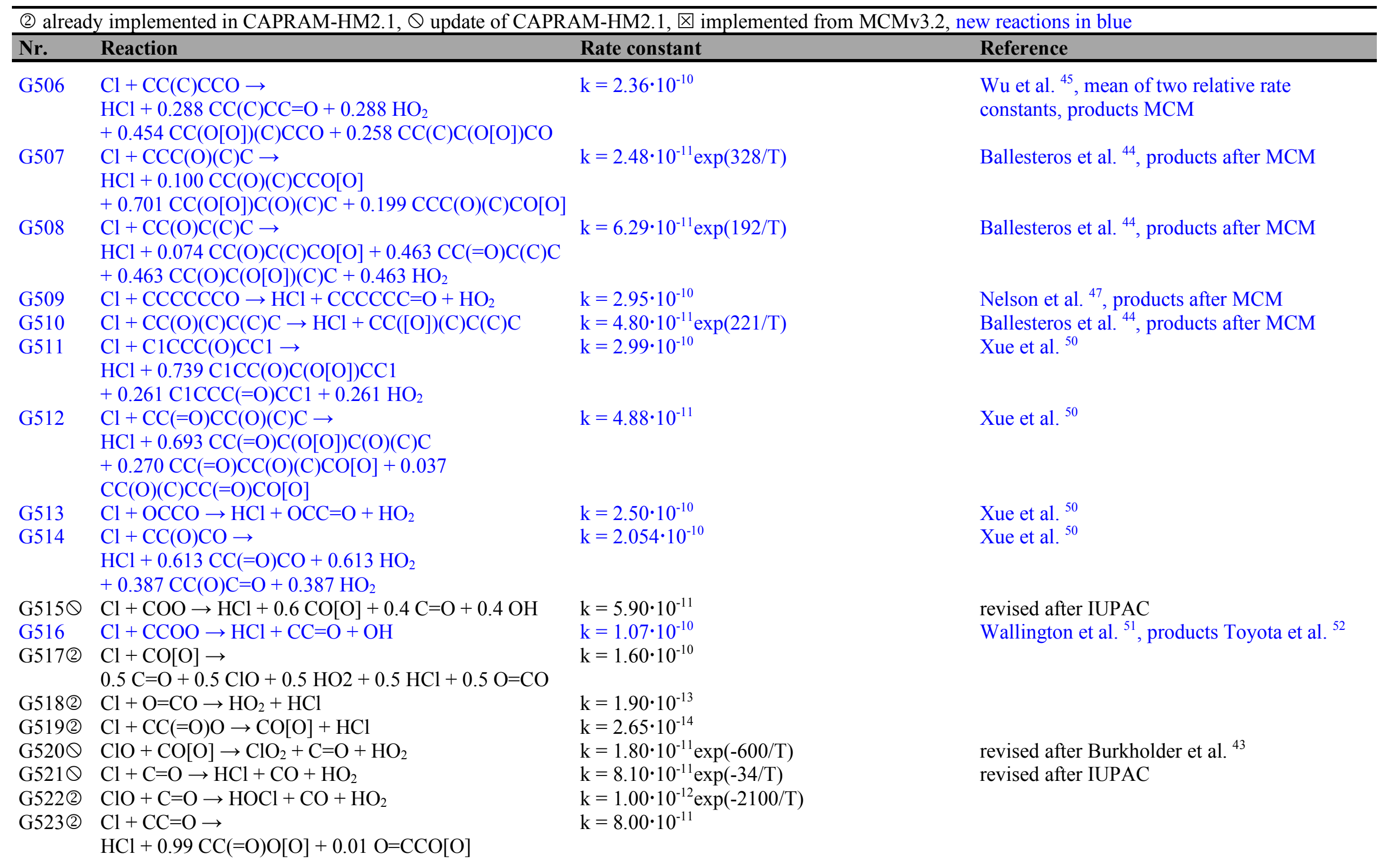




\begin{tabular}{|c|c|c|c|}
\hline Nr. & Reaction & Rate constant & Reference \\
\hline G524(2) & $\mathrm{Cl}+\mathrm{CCC}=\mathrm{O} \rightarrow \mathrm{HCl}+\mathrm{CCC}(=\mathrm{O}) \mathrm{O}[\mathrm{O}]$ & $\mathrm{k}=1.30 \cdot 10^{-10}$ & \\
\hline G525 & $\begin{array}{l}\mathrm{Cl}+\mathrm{CCCC}=\mathrm{O} \rightarrow \\
\mathrm{HCl}+0.34 \mathrm{CC}(\mathrm{O}[\mathrm{O}]) \mathrm{CC}=\mathrm{O}+0.66 \mathrm{CCCC}(=\mathrm{O}) \mathrm{O}[\mathrm{O}]\end{array}$ & $\mathrm{k}=1.63 \cdot 10^{-10}$ & Singh et al. ${ }^{53}$, products after MCM \\
\hline G526 & $\begin{array}{l}\mathrm{Cl}+\mathrm{CC}(\mathrm{C}) \mathrm{C}=\mathrm{O} \rightarrow \\
\mathrm{HCl}+0.12 \mathrm{CC}(\mathrm{C}=\mathrm{O}) \mathrm{CO}[\mathrm{O}]+0.03 \mathrm{CC}(\mathrm{O}[\mathrm{O}])(\mathrm{C}) \mathrm{C}=\mathrm{O} \\
+0.85 \mathrm{CC}(\mathrm{C}) \mathrm{C}(=\mathrm{O}) \mathrm{O}[\mathrm{O}]\end{array}$ & $\mathrm{k}=1.70 \cdot 10^{-10}$ & Thevenet et al. ${ }^{54}$, products Le Crane et al. ${ }^{55}$ \\
\hline G527 & $\begin{array}{l}\mathrm{Cl}+\mathrm{CCCCC}=\mathrm{O} \rightarrow \\
\mathrm{HCl}+0.55 \mathrm{CCC}(\mathrm{O}[\mathrm{O}]) \mathrm{CC}=\mathrm{O} \\
+0.45 \mathrm{CCCCC}(=\mathrm{O}) \mathrm{O}[\mathrm{O}]\end{array}$ & $\mathrm{k}=2.56 \cdot 10^{-10}$ & $\begin{array}{l}\text { Rodriguez et al. }{ }^{56} \text {, products MCM, yields } \\
\text { Singh et al. }{ }^{53}\end{array}$ \\
\hline G528 & $\mathrm{Cl}+\mathrm{CC}(\mathrm{C})(\mathrm{C}) \mathrm{C}=\mathrm{O} \rightarrow \mathrm{HCl}+\mathrm{CC}(\mathrm{C})(\mathrm{C}) \mathrm{C}(=\mathrm{O}) \mathrm{O}[\mathrm{O}]$ & $\mathrm{k}=1.20 \cdot 10^{-10}$ & Ullerstam et al. ${ }^{57}$, products after MCM \\
\hline G529 & $\mathrm{Cl}+\mathrm{CCCCCC}=\mathrm{O} \rightarrow \mathrm{HCl}+\mathrm{CCCCCC}(=\mathrm{O}) \mathrm{O}[\mathrm{O}]$ & $\mathrm{k}=2.88 \cdot 10^{-10}$ & Rodriguez et al. ${ }^{56}$ \\
\hline G530 & $\mathrm{Cl}+\mathrm{CCCCCCC}=\mathrm{O} \rightarrow \mathrm{HCl}+\mathrm{CCCCCCC}(=\mathrm{O}) \mathrm{O}[\mathrm{O}]$ & $\mathrm{k}=3.00 \cdot 10^{-10}$ & Rodriguez et al. ${ }^{56}$ \\
\hline G531 (2) & $\mathrm{Cl}+\mathrm{CC}(=\mathrm{O}) \mathrm{C}=\mathrm{O} \rightarrow \mathrm{HCl}+\mathrm{CC}(=\mathrm{O}) \mathrm{C}(=\mathrm{O}) \mathrm{O}[\mathrm{O}]$ & $\mathrm{k}=4.80 \cdot 10^{-11}$ & \\
\hline G532(2) & $\mathrm{Cl}+\mathrm{O}=\mathrm{CC}=\mathrm{O} \rightarrow \mathrm{HCl}+2.0 \mathrm{CO}+\mathrm{HO}_{2}$ & $\mathrm{k}=3.80 \cdot 10^{-11}$ & \\
\hline G533 & $\mathrm{Cl}+\mathrm{O}=\mathrm{CC}=\mathrm{CC}=\mathrm{O} \rightarrow \mathrm{O}=\mathrm{CC}=\mathrm{CC}(=\mathrm{O}) \mathrm{O}[\mathrm{O}]+\mathrm{HCl}$ & $\mathrm{k}=1.35 \cdot 10^{-10}$ & Martín et al. ${ }^{58}$, products $\mathrm{NO}_{3}$ reaction \\
\hline G534 & $\begin{array}{l}\mathrm{Cl}+\mathrm{O}=\mathrm{CC}=\mathrm{C} \rightarrow \\
0.22 \mathrm{HCl}+0.22 \mathrm{C}=\mathrm{CC}(=\mathrm{O}) \mathrm{O}[\mathrm{O}] \\
+0.78 \mathrm{ClCC}(\mathrm{O}[\mathrm{O}]) \mathrm{C}=\mathrm{O}\end{array}$ & $\mathrm{k}=2.20 \cdot 10^{-10}$ & Canosa-Mas et al. ${ }^{59}$ \\
\hline G535 & $\mathrm{ClCC}(\mathrm{O}[\mathrm{O}]) \mathrm{C}=\mathrm{O}+\mathrm{HO}_{2} \rightarrow \mathrm{ClCC}(\mathrm{OO}) \mathrm{C}=\mathrm{O}$ & $\mathrm{k}=1.51 \cdot 10^{-13} \exp (1300 / \mathrm{T})$ & after MCM \\
\hline G536 & $\begin{array}{l}\mathrm{ClCC}(\mathrm{O}[\mathrm{O}]) \mathrm{C}=\mathrm{O}+\mathrm{NO} \rightarrow \\
\mathrm{ClCC}=\mathrm{O}+\mathrm{HO}_{2}+\mathrm{CO}+\mathrm{NO}_{2}\end{array}$ & $\mathrm{k}=2.70 \cdot 10^{-12} \exp (360 / \mathrm{T})$ & $\begin{array}{l}\text { Rate constant MCM, products Canosa-Mas et } \\
\text { al. }{ }^{9}\end{array}$ \\
\hline G537 & $\begin{array}{l}\mathrm{ClCC}(\mathrm{O}[\mathrm{O}]) \mathrm{C}=\mathrm{O}+\mathrm{NO}_{3} \rightarrow \\
\mathrm{ClCC}=\mathrm{O}+\mathrm{HO}_{2}+\mathrm{CO}+\mathrm{NO}_{2}\end{array}$ & $\mathrm{k}=2.30 \cdot 10^{-12}$ & $\begin{array}{l}\text { Rate constant MCM, productsCanosa-Mas et } \\
\text { al. }{ }^{9}\end{array}$ \\
\hline G538 & $\mathrm{ClCC}(\mathrm{O}[\mathrm{O}]) \mathrm{C}=\mathrm{O} \rightarrow \mathrm{ClCC}=\mathrm{O}+\mathrm{HO}_{2}+\mathrm{CO}$ & $\mathrm{k}=8.80 \cdot 10^{-13} * \mathrm{RO}_{2}$ & $\begin{array}{l}\text { Rate constant MCM, products Canosa-Mas et } \\
\text { al. }{ }^{99}\end{array}$ \\
\hline G539 & $\mathrm{ClCC}(\mathrm{OO}) \mathrm{C}=\mathrm{O}+\mathrm{OH} \rightarrow \mathrm{ClCC}(=\mathrm{O}) \mathrm{C}=\mathrm{O}+\mathrm{OH}$ & $\mathrm{k}=4.77 \cdot 10^{-11}$ & after MCM \\
\hline G540 & $\mathrm{ClCC}(\mathrm{OO}) \mathrm{C}=\mathrm{O} \rightarrow \mathrm{ClCC}=\mathrm{O}+\mathrm{HO}_{2}+\mathrm{CO}+\mathrm{OH}$ & $\mathrm{J}=7.649 \cdot 10^{-06 *} \cos (\chi)^{0.682} \exp (-0.279 / \cos (\chi))$ & after MCM \\
\hline G541 & $\begin{array}{l}\mathrm{Cl}+\mathrm{CC}(\mathrm{C}=\mathrm{O})=\mathrm{C} \rightarrow \\
0.2 \mathrm{C}=\mathrm{C}(\mathrm{C}) \mathrm{C}(=\mathrm{O}) \mathrm{O}[\mathrm{O}]+0.8 \mathrm{CC}(\mathrm{O}[\mathrm{O}])(\mathrm{CCl}) \mathrm{C}=\mathrm{O} \\
+0.2 \mathrm{HCl}\end{array}$ & $\mathrm{k}=2.55 \cdot 10^{-10}$ & $\begin{array}{l}\text { Rate constant average Canosa-Mas et al. }{ }^{59} \text {, } \\
\text { Wang et al. }{ }^{60} \text {, Orlando et al. }{ }^{61} \& \text { Kaiser et al. } \\
62 \text {, Branching ratios Hasson et al. }{ }^{63} \text { (consistent } \\
\text { Sun et al. }{ }^{64} \text { ) }\end{array}$ \\
\hline
\end{tabular}




\begin{tabular}{|c|c|c|c|}
\hline Nr. & Reaction & Rate constant & Reference \\
\hline G542 & $\begin{array}{l}\mathrm{CC}(\mathrm{O}[\mathrm{O}])(\mathrm{CCl}) \mathrm{C}=\mathrm{O}+\mathrm{HO}_{2} \rightarrow \\
0.8 \mathrm{CC}(\mathrm{OO})(\mathrm{CCl}) \mathrm{C}=\mathrm{O}+0.2 \mathrm{CC}(=\mathrm{O}) \mathrm{CCl}+0.2 \mathrm{CO} \\
+0.2 \mathrm{HO} 2+0.2 \mathrm{OH}\end{array}$ & $\mathrm{k}=1.00 \cdot 10^{-11}$ & Hasson et al. ${ }^{63}$, decay of RO Orlando et al. ${ }^{61}$ \\
\hline G543 & $\begin{array}{l}\mathrm{CC}(\mathrm{O}[\mathrm{O}])(\mathrm{CCl}) \mathrm{C}=\mathrm{O}+\mathrm{NO} \rightarrow \\
\mathrm{CC}(=\mathrm{O}) \mathrm{CCl}+\mathrm{CO}+\mathrm{HO}_{2}+\mathrm{NO}_{2}\end{array}$ & $\mathrm{k}=1.17 \cdot 10^{-11}$ & $\underset{61}{\text { Hsin and Elrod }}{ }^{65}$, decay of RO Orlando et al. \\
\hline G544 & $\begin{array}{l}\mathrm{CC}(\mathrm{O}[\mathrm{O}])(\mathrm{CCl}) \mathrm{C}=\mathrm{O} \rightarrow \\
\mathrm{CC}(=\mathrm{O}) \mathrm{CCl}+\mathrm{CO}+\mathrm{HO}_{2}\end{array}$ & $\mathrm{k}=2.40 \cdot 10^{-12} * \mathrm{RO}_{2}$ & Hasson et al. ${ }^{63}$, decay of RO Orlando et al. ${ }^{61}$ \\
\hline G545 & $\mathrm{CC}(\mathrm{OO})(\mathrm{CCl}) \mathrm{C}=\mathrm{O} \rightarrow \mathrm{CC}(=\mathrm{O}) \mathrm{CCl}+\mathrm{CO}+\mathrm{HO}_{2}+\mathrm{OH}$ & $\mathrm{J}=7.914 \cdot 10^{-05 *} \cos (\chi)^{0.764} \exp (-0.364 / \cos (\chi))$ & after MACROOH in MCM \\
\hline G546 & $\mathrm{OH}+\mathrm{CC}(\mathrm{OO})(\mathrm{CCl}) \mathrm{C}=\mathrm{O} \rightarrow \mathrm{CC}(=\mathrm{O}) \mathrm{CCl}+\mathrm{CO}+\mathrm{OH}$ & $\mathrm{k}=3.77 \cdot 10^{-11}$ & after MACROOH in MCM \\
\hline G547 & $\mathrm{Cl}+\mathrm{CC}(=\mathrm{O}) \mathrm{C}=\mathrm{C} \rightarrow \mathrm{CC}(=\mathrm{O}) \mathrm{C}(\mathrm{O}[\mathrm{O}])=\mathrm{CCl}$ & $\mathrm{k}=2.10 \cdot 10^{-10}$ & Canosa-Mas et al. ${ }^{59}$ \\
\hline G548 & $\begin{array}{l}\mathrm{CC}(=\mathrm{O}) \mathrm{C}(\mathrm{O}[\mathrm{O}]) \mathrm{CCl}+\mathrm{HO}_{2} \rightarrow \\
\mathrm{CC}(=\mathrm{O}) \mathrm{C}(\mathrm{OO}) \mathrm{CCl}\end{array}$ & $\mathrm{k}=1.82 \cdot 10^{-13} \exp (1300 / \mathrm{T})$ & after MCM, products Canosa-Mas et al. ${ }^{59}$ \\
\hline G549 & $\begin{array}{l}\mathrm{CC}(=\mathrm{O}) \mathrm{C}(\mathrm{O}[\mathrm{O}]) \mathrm{CCl}+\mathrm{NO} \rightarrow \\
\mathrm{ClCC}=\mathrm{O}+\mathrm{NO}_{2}+\mathrm{CC}(=\mathrm{O}) \mathrm{O}[\mathrm{O}]\end{array}$ & $\mathrm{k}=2.70 \cdot 10^{-12} \exp (360 / \mathrm{T})$ & after MCM, products Canosa-Mas et al. ${ }^{59}$ \\
\hline G550 & $\begin{array}{l}\mathrm{CC}(=\mathrm{O}) \mathrm{C}(\mathrm{O}[\mathrm{O}]) \mathrm{CCl}+\mathrm{NO}_{3} \rightarrow \\
\mathrm{ClCC}=\mathrm{O}+\mathrm{NO}_{2}+\mathrm{CC}(=\mathrm{O}) \mathrm{O}[\mathrm{O}]\end{array}$ & $\mathrm{k}=2.30 \cdot 10^{-12}$ & after MCM, products Canosa-Mas et al. ${ }^{59}$ \\
\hline G551 & $\begin{array}{l}\mathrm{CC}(=\mathrm{O}) \mathrm{C}(\mathrm{O}[\mathrm{O}]) \mathrm{CCl} \rightarrow \\
\mathrm{ClCC}=\mathrm{O}+\mathrm{CC}(=\mathrm{O}) \mathrm{O}[\mathrm{O}]\end{array}$ & $\mathrm{k}=8.80 \cdot 10^{-13} * \mathrm{RO}_{2}$ & after MCM, products Canosa-Mas et al. ${ }^{59}$ \\
\hline G552 & $\mathrm{CC}(=\mathrm{O}) \mathrm{C}(\mathrm{OO}) \mathrm{CCl} \rightarrow \mathrm{ClCC}=\mathrm{O}+\mathrm{CC}(=\mathrm{O}) \mathrm{O}[\mathrm{O}]+\mathrm{OH}$ & $\mathrm{J}=7.649 \cdot 10^{-05 *} \cos (\chi)^{0.682} \exp (-0.279 / \cos (\chi))$ & after MVKOOH in MCM \\
\hline G553 & $\begin{array}{l}\mathrm{OH}+\mathrm{CC}(=\mathrm{O}) \mathrm{C}(\mathrm{OO}) \mathrm{CCl} \rightarrow \\
\mathrm{ClCC}=\mathrm{O}+\mathrm{CC}(=\mathrm{O}) \mathrm{O}[\mathrm{O}]+\mathrm{OH}\end{array}$ & $\mathrm{k}=3.95 \cdot 10^{-11}$ & after $\mathrm{MVKOOH}$ in $\mathrm{MCM}$ \\
\hline G554(2) & $\mathrm{Cl}+\mathrm{CC}(=\mathrm{O}) \mathrm{C} \rightarrow \mathrm{HCl}+\mathrm{CC}(=\mathrm{O}) \mathrm{CO}[\mathrm{O}]$ & $\mathrm{k}=3.20 \cdot 10^{-11} \exp (-815 / \mathrm{T})$ & \\
\hline G555@ & $\begin{array}{l}\mathrm{Cl}+\mathrm{CCC}(=\mathrm{O}) \mathrm{C} \rightarrow \\
\mathrm{HCl}+0.22 \mathrm{CC}(=\mathrm{O}) \mathrm{CCO}[\mathrm{O}]+0.75 \mathrm{CC}(=\mathrm{O}) \mathrm{C}(\mathrm{O}[\mathrm{O}]) \mathrm{C} \\
+0.03 \mathrm{CCC}(=\mathrm{O}) \mathrm{CO}[\mathrm{O}]\end{array}$ & $\mathrm{k}=3.05 \cdot 10^{-11} \exp (80 / \mathrm{T})$ & $\begin{array}{l}\text { revised after IUPAC, products after Kaiser et } \\
\text { al. }{ }_{6}\end{array}$ \\
\hline G556 & $\begin{array}{l}\mathrm{Cl}+\mathrm{CCCC}(=\mathrm{O}) \mathrm{C} \rightarrow \\
0.82 \mathrm{CC}(=\mathrm{O}) \mathrm{CC}(\mathrm{O}[\mathrm{O}]) \mathrm{C}+0.18 \mathrm{CCC}(\mathrm{O}[\mathrm{O}]) \mathrm{C}(=\mathrm{O}) \mathrm{C} \\
+\mathrm{HCl}\end{array}$ & $\mathrm{k}=1.11 \cdot 10^{-10}$ & Taketani et al. ${ }^{67}$ \\
\hline G557 & $\begin{array}{l}\mathrm{Cl}+\mathrm{CCC}(=\mathrm{O}) \mathrm{CC} \rightarrow \\
0.79 \mathrm{CCC}(=\mathrm{O}) \mathrm{C}(\mathrm{O}[\mathrm{O}]) \mathrm{C}+0.21 \mathrm{CCC}(=\mathrm{O}) \mathrm{CCO}[\mathrm{O}] \\
+\mathrm{HCl}\end{array}$ & $\mathrm{k}=5.66 \cdot 10^{-11} \exp (87 / \mathrm{T})$ & Zhao et al. ${ }^{68}$, products Kaiser et al. ${ }^{62}$ \\
\hline
\end{tabular}




\begin{tabular}{|c|c|c|c|}
\hline Nr. & Reaction & Rate constant & Reference \\
\hline G558 & $\begin{array}{l}\mathrm{Cl}+\mathrm{CC}(=\mathrm{O}) \mathrm{C}(\mathrm{C}) \mathrm{C} \rightarrow \\
\mathrm{HCl}+0.523 \mathrm{CC}(=\mathrm{O}) \mathrm{C}(\mathrm{O}[\mathrm{O}])(\mathrm{C}) \mathrm{C} \\
+0.477 \mathrm{CC}(=\mathrm{O}) \mathrm{C}(\mathrm{C}) \mathrm{CO}[\mathrm{O}]\end{array}$ & $\mathrm{k}=6.80 \cdot 10^{-11}$ & Farrugia et al. ${ }^{69}$, products after MCM \\
\hline G559 & $\begin{array}{l}\mathrm{Cl}+\mathrm{CCCCC}(=\mathrm{O}) \mathrm{C} \rightarrow \\
\mathrm{HCl}+0.715 \mathrm{CC}(=\mathrm{O}) \mathrm{CC}(\mathrm{O}[\mathrm{O}]) \mathrm{CC} \\
+0.162 \mathrm{CC}(=\mathrm{O}) \mathrm{CCC}(\mathrm{O}[\mathrm{O}]) \mathrm{C} \\
+0.123 \mathrm{CCCC}(\mathrm{O}[\mathrm{O}]) \mathrm{C}(=\mathrm{O}) \mathrm{C}\end{array}$ & $\mathrm{k}=1.88 \cdot 10^{-10}$ & Taketani et al. ${ }^{67}$, products after MCM \\
\hline G560 & $\begin{array}{l}\mathrm{Cl}+\mathrm{CCCC}(=\mathrm{O}) \mathrm{CC} \rightarrow \\
\mathrm{HCl}+0.638 \mathrm{CCC}(=\mathrm{O}) \mathrm{CC}(\mathrm{O}[\mathrm{O}]) \mathrm{C} \\
+0.142 \mathrm{CCC}(=\mathrm{O}) \mathrm{C}(\mathrm{O}[\mathrm{O}]) \mathrm{CC} \\
+0.110 \mathrm{CCCC}(=\mathrm{O}) \mathrm{C}(\mathrm{O}[\mathrm{O}]) \mathrm{C} \\
+0.110 \mathrm{CCCC}(=\mathrm{O}) \mathrm{CCO}[\mathrm{O}]\end{array}$ & $\mathrm{k}=1.43 \cdot 10^{-10}$ & Taketani et al. ${ }^{67}$, products after MCM \\
\hline G561 & $\begin{array}{l}\mathrm{Cl}+\mathrm{CC}(=\mathrm{O}) \mathrm{CC}(\mathrm{C}) \mathrm{C} \rightarrow \\
\mathrm{HCl}+0.91 \mathrm{CC}(=\mathrm{O}) \mathrm{CC}(\mathrm{O}[\mathrm{O}])(\mathrm{C}) \mathrm{C} \\
+0.09 \mathrm{CC}(=\mathrm{O}) \mathrm{C}(\mathrm{O}[\mathrm{O}]) \mathrm{C}(\mathrm{C}) \mathrm{C}\end{array}$ & $\mathrm{k}=1.10 \cdot 10^{-10}$ & Farrugia et al. ${ }^{69}$, products after MCM \\
\hline G562 & $\begin{array}{l}\mathrm{Cl}+\mathrm{CC}(=\mathrm{O}) \mathrm{C}(\mathrm{C})(\mathrm{C}) \mathrm{C} \rightarrow \\
\mathrm{HCl}+\mathrm{CC}(=\mathrm{O}) \mathrm{C}(\mathrm{C})(\mathrm{C}) \mathrm{CO}[\mathrm{O}]\end{array}$ & $\mathrm{k}=4.80 \cdot 10^{-11}$ & Farrugia et al. ${ }^{69}$, products after MCM \\
\hline G563 & $\begin{array}{l}\mathrm{Cl}+\mathrm{CC}(=\mathrm{O}) \mathrm{CCC}(\mathrm{C}) \mathrm{C} \rightarrow \\
\mathrm{HCl}+\mathrm{CC}(=\mathrm{O}) \mathrm{CC}(\mathrm{O}[\mathrm{O}]) \mathrm{C}(\mathrm{C}) \mathrm{C}\end{array}$ & $\mathrm{k}=1.65 \cdot 10^{-10}$ & Farrugia et al. ${ }^{69}$, products after MCM \\
\hline G564 & $\begin{array}{l}\mathrm{Cl}+\mathrm{CCC}(\mathrm{C}) \mathrm{C}(=\mathrm{O}) \mathrm{C} \rightarrow \\
\mathrm{HCl}+0.671 \mathrm{CC}(\mathrm{O}[\mathrm{O}]) \mathrm{C}(\mathrm{C}) \mathrm{C}(=\mathrm{O}) \mathrm{C} \\
+0.329 \mathrm{CCC}(\mathrm{O}[\mathrm{O}])(\mathrm{C}) \mathrm{C}(=\mathrm{O}) \mathrm{C}\end{array}$ & $\mathrm{k}=9.40 \cdot 10^{-11}$ & Farrugia et al. ${ }^{69}$, products after MCM \\
\hline G565 & $\begin{array}{l}\mathrm{Cl}+\mathrm{C} 1 \mathrm{CCC}(=\mathrm{O}) \mathrm{CC} 1 \rightarrow \\
\mathrm{HCl}+\mathrm{C} 1 \mathrm{C}(=\mathrm{O}) \mathrm{CC}(\mathrm{O}[\mathrm{O}]) \mathrm{CC} 1\end{array}$ & $\mathrm{k}=1.30 \cdot 10^{-10}$ & Xue et al. ${ }^{50}$ \\
\hline G566 & $\mathrm{Cl}+\mathrm{CC}(=\mathrm{O}) \mathrm{CCl} \rightarrow \mathrm{HCl}+\mathrm{CC}(=\mathrm{O}) \mathrm{C}(\mathrm{Cl}) \mathrm{O}[\mathrm{O}]$ & $\mathrm{k}=3.50 \cdot 10^{-12}$ & Notario et al. ${ }^{70}$ \\
\hline G567 & $\mathrm{Cl}+\mathrm{CC}(=\mathrm{O}) \mathrm{C}(=\mathrm{O}) \mathrm{C} \rightarrow \mathrm{HCl}+\mathrm{CC}(=\mathrm{O}) \mathrm{C}(=\mathrm{O}) \mathrm{CO}[\mathrm{O}]$ & $\mathrm{k}=2.55 \cdot 10^{-11} \exp (-1156 / \mathrm{T})$ & Cuevas et al. ${ }^{71}$ \\
\hline $\begin{array}{l}\text { G568(2) } \\
\text { G569(2) }\end{array}$ & $\begin{array}{l}\mathrm{Cl}+\mathrm{CC}(=\mathrm{O}) \mathrm{CO} \rightarrow \mathrm{HCl}+\mathrm{CC}(=\mathrm{O}) \mathrm{C}=\mathrm{O}+\mathrm{HO}_{2} \\
\mathrm{Cl}+\mathrm{OCC}=\mathrm{O} \rightarrow \mathrm{HCl}+\mathrm{OCC}(=\mathrm{O}) \mathrm{O}[\mathrm{O}]\end{array}$ & $\begin{array}{l}\mathrm{k}=5.70 \cdot 10^{-11} \\
\mathrm{k}=7.00 \cdot 10^{-11}\end{array}$ & \\
\hline G570@ & $\begin{array}{l}\mathrm{Cl}+\mathrm{C} \# \mathrm{C} \rightarrow \\
0.26 \mathrm{O}=\mathrm{CCl}+0.21 \mathrm{Cl}+0.53 \mathrm{HCl}+0.21 \mathrm{O}=\mathrm{CC}=\mathrm{O} \\
+1.32 \mathrm{CO}+0.79 \mathrm{HO}_{2}\end{array}$ & TROE & Burkholder et al. ${ }^{43}$ \\
\hline $\begin{array}{l}\text { G571 (2) } \\
\text { G572(2) }\end{array}$ & $\begin{array}{l}\mathrm{Cl}+\mathrm{CON}(=\mathrm{O})=\mathrm{O} \rightarrow \mathrm{C}=\mathrm{O}+\mathrm{NO}_{2}+\mathrm{HCl} \\
\mathrm{Cl}+\mathrm{CCON}(=\mathrm{O})=\mathrm{O} \rightarrow \mathrm{CC}=\mathrm{O}+\mathrm{NO}_{2}+\mathrm{HCl}\end{array}$ & $\begin{array}{l}\mathrm{k}=2.40 \cdot 10^{-13} \\
\mathrm{k}=4.70 \cdot 10^{-12}\end{array}$ & \\
\hline
\end{tabular}




\begin{tabular}{|c|c|c|c|}
\hline$\overline{\mathrm{Nr} .}$ & Reaction & Rate constant & Reference \\
\hline G573(2) & $\mathrm{Cl}+\mathrm{CCCON}(=\mathrm{O})=\mathrm{O} \rightarrow \mathrm{CCC}=\mathrm{O}+\mathrm{NO}_{2}+\mathrm{HCl}$ & $\mathrm{k}=2.20 \cdot 10^{-11}$ & \\
\hline G574(2) & $\mathrm{Cl}+\mathrm{CC}(\mathrm{ON}(=\mathrm{O})=\mathrm{O}) \mathrm{C} \rightarrow \mathrm{CC}(=\mathrm{O}) \mathrm{C}+\mathrm{NO}_{2}+\mathrm{HCl}$ & $\mathrm{k}=3.80 \cdot 10^{-12}$ & \\
\hline G575(2) & $\mathrm{Cl}+\mathrm{CCCCON}(=\mathrm{O})=\mathrm{O} \rightarrow \mathrm{CCCC}=\mathrm{O}+\mathrm{NO}_{2}+\mathrm{HCl}$ & $\mathrm{k}=8.50 \cdot 10^{-11}$ & \\
\hline G576 & $\mathrm{Cl}+\mathrm{c} 1 \operatorname{ccccc} 1 \mathrm{C} \rightarrow \mathrm{HCl}+\mathrm{c} 1 \operatorname{ccccc} 1 \mathrm{CO}[\mathrm{O}]$ & $\mathrm{k}=6.20 \cdot 10^{-11}$ & average Wang et al. ${ }^{72}$ \\
\hline G577 & $\mathrm{Cl}+\mathrm{Cc} 1 \operatorname{cccc} 1 \mathrm{C} \rightarrow \mathrm{HCl}+\mathrm{Cc} 1 \operatorname{ccccc} 1 \mathrm{CO}[\mathrm{O}]$ & $\mathrm{k}=1.40 \cdot 10^{-10}$ & average Wang et al. ${ }^{72}$ \\
\hline G578 & $\mathrm{Cl}+\mathrm{clc}(\mathrm{C}) \operatorname{ccc} 1 \mathrm{C} \rightarrow \mathrm{Cc} 1 \mathrm{cccc}(\mathrm{c} 1) \mathrm{CO}[\mathrm{O}]+\mathrm{HCl}$ & $\mathrm{k}=1.35 \cdot 10^{-10}$ & average Wang et al. ${ }^{72}$ \\
\hline G579 & $\mathrm{Cl}+\mathrm{clcc}(\mathrm{C}) \operatorname{ccc} 1 \mathrm{C} \rightarrow \mathrm{Cc} 1 \mathrm{ccc}(\mathrm{CO}[\mathrm{O}]) \mathrm{ccl}+\mathrm{HCl}$ & $\mathrm{k}=1.44 \cdot 10^{-10}$ & average Wang et al. ${ }^{72}$ \\
\hline G580 & $\mathrm{Cl}+\mathrm{c} 1 \operatorname{cccc} 1 \mathrm{C}=\mathrm{O} \rightarrow \mathrm{HCl}+\operatorname{cl} \operatorname{ccc} c 1 \mathrm{C}(=\mathrm{O}) \mathrm{O}[\mathrm{O}]$ & $\mathrm{k}=1.00 \cdot 10^{-10}$ & Thiault et al. ${ }^{73}$ \\
\hline G581 & $\mathrm{Cl}+\mathrm{Cc} 1 \operatorname{ccc} c 1 \mathrm{C}=\mathrm{O} \rightarrow \mathrm{HCl}+\mathrm{C} \operatorname{coc} \operatorname{ccc} 1 \mathrm{C}(=\mathrm{O}) \mathrm{O}[\mathrm{O}]$ & $\mathrm{k}=1.90 \cdot 10^{-10}$ & Thiault et al. ${ }^{73}$ \\
\hline G582 & $\begin{array}{l}\mathrm{Cl}+\mathrm{Cc} 1 \operatorname{ccc}(\mathrm{c} 1) \mathrm{C}=\mathrm{O} \rightarrow \\
\mathrm{HCl}+\mathrm{Cc} 1 \operatorname{cccc}(\mathrm{c} 1) \mathrm{C}(=\mathrm{O}) \mathrm{O}[\mathrm{O}]\end{array}$ & $\mathrm{k}=1.70 \cdot 10^{-10}$ & Thiault et al. ${ }^{73}$ \\
\hline G583 & $\begin{array}{l}\mathrm{Cl}+\mathrm{Cc} 1 \operatorname{ccc}(\mathrm{C}=\mathrm{O}) \mathrm{cc} 1 \rightarrow \\
\mathrm{HCl}+\mathrm{Cc} 1 \operatorname{ccc}(\mathrm{C}(=\mathrm{O}) \mathrm{O}[\mathrm{O}]) \mathrm{cc} 1\end{array}$ & $\mathrm{k}=1.40 \cdot 10^{-10}$ & Thiault et al. ${ }^{73}$ \\
\hline G584 & $\begin{array}{l}\mathrm{Cl}+\mathrm{clc}(\mathrm{C}) \mathrm{cc}(\mathrm{C}=\mathrm{O}) \mathrm{cclC} \rightarrow \\
\mathrm{HCl}+\mathrm{clc}(\mathrm{C}) \mathrm{cc}(\mathrm{C}(=\mathrm{O}) \mathrm{O}[\mathrm{O}]) \mathrm{cc} 1 \mathrm{C}\end{array}$ & $\mathrm{k}=9.60 \cdot 10^{-11}$ & El Dib et al. ${ }^{74}$ \\
\hline G585 & $\mathrm{Cl}+\mathrm{c} 1 \mathrm{ccccc} 1 \mathrm{CC} \rightarrow \mathrm{cl} \operatorname{ccccc} 1 \mathrm{CCO}[\mathrm{O}]+\mathrm{HCl}$ & $\mathrm{k}=9.10 \cdot 10^{-11}$ & Xue et al. ${ }^{50}$ \\
\hline G586 & $\mathrm{Cl}+\mathrm{c} 1 \mathrm{ccccc} 1 \mathrm{CCC} \rightarrow \mathrm{c} 1 \operatorname{cccc} 1 \mathrm{C}(\mathrm{O}[\mathrm{O}]) \mathrm{CC}+\mathrm{HCl}$ & $\mathrm{k}=7.50 \cdot 10^{-11}$ & Xue et al. ${ }^{50}$ \\
\hline G587 & $\mathrm{Cl}+\mathrm{c} 1 \operatorname{ccccc} 1 \mathrm{C}(\mathrm{C}) \mathrm{C} \rightarrow \operatorname{cl} \operatorname{coccc} 1 \mathrm{C}(\mathrm{O}[\mathrm{O}])(\mathrm{C}) \mathrm{C}+\mathrm{HCl}$ & $\mathrm{k}=8.20 \cdot 10^{-11}$ & Xue et al. ${ }^{50}$ \\
\hline G588 & $\mathrm{Cl}+\mathrm{Cc} 1 \operatorname{cccc}(\mathrm{C}) \mathrm{c} 1 \mathrm{C} \rightarrow \mathrm{Cc} 1 \operatorname{ccc}(\mathrm{CO}[\mathrm{O}]) \mathrm{c} 1 \mathrm{C}+\mathrm{HCl}$ & $\mathrm{k}=3.60 \cdot 10^{-10}$ & Xue et al. ${ }^{50}$ \\
\hline G589 & $\mathrm{Cl}+\mathrm{Cc} 1 \mathrm{ccc}(\mathrm{C}) \mathrm{cc} 1 \mathrm{C} \rightarrow \mathrm{Cc} 1 \mathrm{ccc}(\mathrm{CO}[\mathrm{O}]) \mathrm{cc} 1 \mathrm{C}+\mathrm{HCl}$ & $\mathrm{k}=3.60 \cdot 10^{-10}$ & Xue et al. ${ }^{50}$ \\
\hline G590 & $\mathrm{Cl}+\mathrm{clc}(\mathrm{C}) \mathrm{cc}(\mathrm{C}) \mathrm{cclC} \rightarrow \mathrm{clc}(\mathrm{C}) \mathrm{cc}(\mathrm{CO}[\mathrm{O}]) \mathrm{ccl} \mathrm{C}+\mathrm{HCl}$ & $\mathrm{k}=2.42 \cdot 10^{-10}$ & Wang et al. ${ }^{72}$ \\
\hline G591 & $\mathrm{Cl}+\mathrm{Cc} 1 \operatorname{ccc} c 1 \mathrm{CC} \rightarrow \mathrm{Cc} 1 \operatorname{cccc} 1 \mathrm{C}(\mathrm{O}[\mathrm{O}]) \mathrm{C}+\mathrm{HCl}$ & $\mathrm{k}=1.10 \cdot 10^{-10}$ & Xue et al. ${ }^{50}$ \\
\hline G592 & $\mathrm{Cl}+\mathrm{c} 1 \mathrm{c}(\mathrm{C}) \operatorname{ccc} 1 \mathrm{CC} \rightarrow \mathrm{Cc} 1 \operatorname{cccc} 1 \mathrm{C}(\mathrm{O}[\mathrm{O}]) \mathrm{C}+\mathrm{HCl}$ & $\mathrm{k}=1.40 \cdot 10^{-10}$ & Xue et al. ${ }^{50}$ \\
\hline G593 & $\mathrm{Cl}+\mathrm{cl} \operatorname{cc}(\mathrm{C}) \operatorname{ccc} 1 \mathrm{CC} \rightarrow \mathrm{Cc} 1 \mathrm{ccccc} 1 \mathrm{C}(\mathrm{O}[\mathrm{O}]) \mathrm{C}+\mathrm{HCl}$ & $\mathrm{k}=2.20 \cdot 10^{-10}$ & Xue et al. ${ }^{50}$ \\
\hline G594 & $\mathrm{Cl}+\mathrm{c} 1 \operatorname{ccccc} 1 \mathrm{C}=\mathrm{C} \rightarrow \operatorname{cl} \operatorname{ccccc} 1 \mathrm{C}(\mathrm{Cl}) \mathrm{CO}[\mathrm{O}]$ & $\mathrm{k}=3.60 \cdot 10^{-10}$ & Shi and Bernhard ${ }^{75}$ \\
\hline G595 & $\begin{array}{l}\operatorname{clcccc} 1 \mathrm{c}(\mathrm{Cl}) \mathrm{CO}[\mathrm{O}]+\mathrm{NO} \rightarrow \\
\operatorname{clcccc} 1 \mathrm{C}(\mathrm{Cl}) \mathrm{C}[\mathrm{O}]+\mathrm{NO}_{2}\end{array}$ & $\mathrm{k}=2.70 \cdot 10^{-12} \exp (360 / \mathrm{T})$ & after $\mathrm{OH}$ reaction in $\mathrm{MCM}$ \\
\hline G596 & $\mathrm{c} 1 \operatorname{ccccc} 1 \mathrm{C}(\mathrm{Cl}) \mathrm{CO}[\mathrm{O}] \rightarrow \operatorname{cl} \operatorname{ccccc} 1 \mathrm{C}(\mathrm{Cl}) \mathrm{C}[\mathrm{O}]$ & $\mathrm{k}=2.50 \cdot 10^{-13}$ & after $\mathrm{OH}$ reaction in $\mathrm{MCM}$ \\
\hline G597 & $\operatorname{c1} \operatorname{ccc} c \operatorname{c} C(\mathrm{Cl}) \mathrm{C}[\mathrm{O}] \rightarrow \mathrm{ClO}+\mathrm{C}=\mathrm{O}+\operatorname{c} 1 \operatorname{ccc} c 1 \mathrm{C}=\mathrm{O}$ & $\mathrm{k}=1.00 \cdot 10^{+06}$ & after $\mathrm{OH}$ reaction in $\mathrm{MCM}$ \\
\hline G598 & $\begin{array}{l}\mathrm{Cl}+\mathrm{c} 1 \mathrm{cc}(\mathrm{O}) \mathrm{c}(\mathrm{N}(=\mathrm{O})=\mathrm{O}) \mathrm{cc} 1 \rightarrow \\
0.4 \mathrm{HCl}+0.4 \mathrm{clcc}(\mathrm{N}(=\mathrm{O})=\mathrm{O}) \mathrm{c}([\mathrm{O}]) \mathrm{cc} 1 \\
+0.6 \mathrm{clcc}(\mathrm{O}) \mathrm{c}(\mathrm{Cl}) \mathrm{cc} 1+0.6 \mathrm{NO}_{2}\end{array}$ & $\mathrm{k}=6.80 \cdot 10^{-12}$ & Bejan et al. ${ }^{76}$ \\
\hline
\end{tabular}




\begin{tabular}{|c|c|c|c|}
\hline Nr. & Reaction & Rate constant & Reference \\
\hline G599 & $\begin{array}{l}\mathrm{Cl}+\mathrm{c} 1 \mathrm{ccc}(\mathrm{N}(=\mathrm{O})=\mathrm{O}) \mathrm{c}(\mathrm{O}) \mathrm{c} 1 \mathrm{C} \rightarrow \\
\mathrm{c} 1 \operatorname{ccc}(\mathrm{N}(=\mathrm{O})=\mathrm{O}) \mathrm{c}([\mathrm{O}]) \mathrm{c} 1 \mathrm{C}+\mathrm{HCl}\end{array}$ & $\mathrm{k}=2.68 \cdot 10^{-11}$ & Bejan et al. ${ }^{76}$, products after MCM \\
\hline G600 & $\mathrm{Cl}+\operatorname{c1ccc}(\mathrm{O}) \mathrm{c} 1 \mathrm{C} \rightarrow \mathrm{HCl}+\operatorname{clccc}([\mathrm{O}]) \mathrm{c} 1 \mathrm{C}$ & $\mathrm{k}=1.10 \cdot 10^{-10}$ & Bejan et al. ${ }^{76}$, products after MCM \\
\hline G601 & $\mathrm{Cl}+\mathrm{c} 1 \mathrm{ccc}(\mathrm{O}) \mathrm{cc} 1 \rightarrow \mathrm{HCl}+\mathrm{clccc}([\mathrm{O}]) \mathrm{cc} 1$ & $\mathrm{k}=1.93 \cdot 10^{-10}$ & Jenkin et al. ${ }^{77}$ \\
\hline G602 & $\mathrm{Cl}+\mathrm{c} 1 \mathrm{cc}(\mathrm{O}) \mathrm{c}(\mathrm{O}) \mathrm{cc} 1 \rightarrow \mathrm{HCl}+\mathrm{clcc}(\mathrm{O}) \mathrm{c}([\mathrm{O}]) \mathrm{cc} 1$ & $\mathrm{k}=6.40 \cdot 10^{-10}$ & Bejan et al. ${ }^{76}$ \\
\hline G603 & $\mathrm{Cl}+\mathrm{COC} \rightarrow \mathrm{HCl}+\mathrm{COCO}[\mathrm{O}]$ & $\mathrm{k}=1.80 \cdot 10^{-10}$ & Jenkin et al. ${ }^{77}$ \\
\hline G604 & $\mathrm{Cl}+\mathrm{COCOO} \rightarrow \mathrm{HCl}+\mathrm{COC}=\mathrm{O}+\mathrm{OH}$ & $\mathrm{k}=6.10 \cdot 10^{-11}$ & $\begin{array}{l}\text { Wallington et al. }{ }^{78} \text {, Products after } \mathrm{OH} \text { reaction } \\
\text { in } \mathrm{MCM}\end{array}$ \\
\hline G605 & $\mathrm{Cl}+\mathrm{COC}=\mathrm{O} \rightarrow \mathrm{HCl}+0.55 \mathrm{C}[\mathrm{O}]+0.45 \mathrm{O}=\mathrm{COCO}[\mathrm{O}]$ & $\mathrm{k}=1.70 \cdot 10^{-11} \exp (-810 / \mathrm{T})$ & Bravo et al. ${ }^{79}$, products Wallington et al. ${ }^{80}$ \\
\hline G606 & $\begin{array}{l}\mathrm{Cl}+\mathrm{CCOC}=\mathrm{O} \rightarrow \\
\mathrm{HCl}+0.096 \mathrm{CCO}[\mathrm{O}]+0.904 \mathrm{CC}(\mathrm{O}[\mathrm{O}]) \mathrm{OC}=\mathrm{O}\end{array}$ & $\mathrm{k}=5.50 \cdot 10^{-11} \exp (-556 / \mathrm{T})$ & Bravo et al. ${ }^{79}$, Products $\mathrm{OH}$ reaction $\mathrm{MCM}$ \\
\hline G607(2) & $\mathrm{Cl}+\mathrm{C}=\mathrm{C} \rightarrow \mathrm{ClCCO}[\mathrm{O}]$ & TROEF & \\
\hline G608@ & $\begin{array}{l}\mathrm{Cl}+\mathrm{CC}=\mathrm{C} \rightarrow \\
0.4 \mathrm{CC}(\mathrm{O}[\mathrm{O}]) \mathrm{CCl}+0.5 \mathrm{CC}(\mathrm{Cl}) \mathrm{CO}[\mathrm{O}] \\
+0.1 \mathrm{C}=\mathrm{CCO}[\mathrm{O}]\end{array}$ & $\mathrm{k}=1.43 \cdot 10^{-14} \exp (2886 / \mathrm{T})$ & $\begin{array}{l}\text { Ceacero-Vega et al. }{ }^{81} \text {, products after Riedel et } \\
\text { al. }{ }^{82}\end{array}$ \\
\hline G609 & $\mathrm{CC}(\mathrm{O}[\mathrm{O}]) \mathrm{CCl}+\mathrm{NO} \rightarrow \mathrm{CC}([\mathrm{O}]) \mathrm{CCl}+\mathrm{NO}_{2}$ & $\mathrm{k}=2.70 \cdot 10^{-12} \exp (360 / \mathrm{T})$ & rate after MCM \\
\hline G610 & $\mathrm{CC}(\mathrm{Cl}) \mathrm{CO}[\mathrm{O}]+\mathrm{NO} \rightarrow \mathrm{CC}(\mathrm{Cl}) \mathrm{C}=\mathrm{O}+\mathrm{NO}_{2}+\mathrm{HO}_{2}$ & $\mathrm{k}=2.70 \cdot 10^{-12} \exp (360 / \mathrm{T})$ & after MCM \\
\hline G611 & $\mathrm{C}=\mathrm{CCO}[\mathrm{O}]+\mathrm{NO} \rightarrow \mathrm{O}=\mathrm{CC}=\mathrm{C}+\mathrm{NO}_{2}$ & $\mathrm{k}=2.70 \cdot 10^{-12} \exp (360 / \mathrm{T})$ & Riedel et al. ${ }^{82}$ \\
\hline G612(2) & $\begin{array}{l}\mathrm{CC}(\mathrm{O}[\mathrm{O}]) \mathrm{CCl} \rightarrow \\
0.2 \mathrm{CC}(\mathrm{O}) \mathrm{CCl}+0.2 \mathrm{CC}(=\mathrm{O}) \mathrm{CCl}+0.6 \mathrm{CC}([\mathrm{O}]) \mathrm{CCl}\end{array}$ & $\mathrm{k}=4.00 \cdot 10^{-14} * \mathrm{RO}_{2}$ & \\
\hline G613 & $\mathrm{CC}(\mathrm{Cl}) \mathrm{C}=\mathrm{O}+\mathrm{OH} \rightarrow \mathrm{CC}(\mathrm{Cl}) \mathrm{C}(=\mathrm{O}) \mathrm{O}[\mathrm{O}]$ & $\mathrm{k}=4.90 \cdot 10^{-12} \exp (405 / \mathrm{T})$ & Riedel et al. ${ }^{82}$ \\
\hline G614 & $\mathrm{CC}(\mathrm{Cl}) \mathrm{C}=\mathrm{O}+\mathrm{NO}_{3} \rightarrow \mathrm{CC}(\mathrm{Cl}) \mathrm{C}(=\mathrm{O}) \mathrm{O}[\mathrm{O}]+\mathrm{HNO}_{3}$ & $\mathrm{k}=3.24 \cdot 10^{-12} \exp (-1860 / \mathrm{T})$ & Riedel et al. ${ }^{82}$ \\
\hline G615 & $\mathrm{CC}(\mathrm{Cl}) \mathrm{C}=\mathrm{O} \rightarrow \mathrm{CC}(\mathrm{Cl}) \mathrm{O}[\mathrm{O}]+\mathrm{HO}_{2}+\mathrm{CO}$ & $\mathrm{J}=2.879 \cdot 10^{-05 *} \cos (\chi)^{1.067} \exp (-0.358 / \cos (\chi))$ & Riedel et al. ${ }^{82}$ \\
\hline G616 & $\begin{array}{l}\mathrm{CC}(\mathrm{Cl}) \mathrm{C}(=\mathrm{O}) \mathrm{O}[\mathrm{O}]+\mathrm{HO}_{2} \rightarrow \\
0.15 \mathrm{CC}(\mathrm{Cl}) \mathrm{C}(=\mathrm{O}) \mathrm{O}+0.15 \mathrm{O}_{3}+0.41 \mathrm{CC}(\mathrm{Cl}) \mathrm{C}(=\mathrm{O}) \mathrm{OO} \\
+0.44 \mathrm{CC}(\mathrm{Cl}) \mathrm{O}[\mathrm{O}]+0.44 \mathrm{OH}\end{array}$ & $\mathrm{k}=5.20 \cdot 10^{-13} \exp (980 / \mathrm{T})$ & Riedel et al. ${ }^{82}$ \\
\hline G617 & $\mathrm{CC}(\mathrm{Cl}) \mathrm{C}(=\mathrm{O}) \mathrm{O}[\mathrm{O}]+\mathrm{NO} \rightarrow \mathrm{CC}(\mathrm{Cl}) \mathrm{O}[\mathrm{O}]+\mathrm{NO}_{2}$ & $\mathrm{k}=7.50 \cdot 10^{-12} \exp (290 / \mathrm{T})$ & Riedel et al. ${ }^{82}$ \\
\hline G618 & $\begin{array}{l}\mathrm{CC}(\mathrm{Cl}) \mathrm{C}(=\mathrm{O}) \mathrm{O}[\mathrm{O}]+\mathrm{NO}_{2} \rightarrow \\
\mathrm{CC}(\mathrm{Cl}) \mathrm{C}(=\mathrm{O}) \mathrm{OON}(=\mathrm{O})=\mathrm{O}\end{array}$ & TROEMCM & Riedel et al. ${ }^{82}$ \\
\hline G619 & $\begin{array}{l}\mathrm{CC}(\mathrm{Cl}) \mathrm{C}(=\mathrm{O}) \mathrm{OON}(=\mathrm{O})=\mathrm{O} \rightarrow \\
\mathrm{CC}(\mathrm{Cl}) \mathrm{C}(=\mathrm{O}) \mathrm{O}[\mathrm{O}]+\mathrm{NO}_{2}\end{array}$ & TROEMCM & after MCM \\
\hline G620 & $\mathrm{CC}(\mathrm{Cl}) \mathrm{C}(=\mathrm{O}) \mathrm{O}[\mathrm{O}]+\mathrm{NO}_{3} \rightarrow \mathrm{CC}(\mathrm{Cl}) \mathrm{O}[\mathrm{O}]+\mathrm{NO}_{2}$ & $\mathrm{k}=4.00 \cdot 10^{-12}$ & Riedel et al. ${ }^{82}$ \\
\hline
\end{tabular}




\begin{tabular}{|c|c|c|c|}
\hline $\mathrm{Nr}$. & Reaction & Rate constant & Reference \\
\hline G621 & $\begin{array}{l}\mathrm{CC}(\mathrm{Cl}) \mathrm{C}(=\mathrm{O}) \mathrm{O}[\mathrm{O}] \rightarrow \\
0.3 \mathrm{CC}(\mathrm{Cl}) \mathrm{C}(=\mathrm{O}) \mathrm{O}+0.7 \mathrm{CC}(\mathrm{Cl}) \mathrm{O}[\mathrm{O}]\end{array}$ & $\mathrm{k}=1.00 \cdot 10^{-11} * \mathrm{RO}_{2}$ & Riedel et al. ${ }^{82}$ \\
\hline G622 & $\mathrm{CC}(\mathrm{Cl}) \mathrm{C}(=\mathrm{O}) \mathrm{OO}+\mathrm{OH} \rightarrow \mathrm{CC}(\mathrm{Cl}) \mathrm{C}(=\mathrm{O}) \mathrm{O}[\mathrm{O}]$ & $\mathrm{k}=4.42 \cdot 10^{-12}$ & Riedel et al. ${ }^{82}$ \\
\hline G623 & $\mathrm{CC}(\mathrm{Cl}) \mathrm{C}(=\mathrm{O}) \mathrm{O}+\mathrm{OH} \rightarrow \mathrm{CC}(\mathrm{Cl}) \mathrm{O}[\mathrm{O}]$ & $\mathrm{k}=1.20 \cdot 10^{-12}$ & Riedel et al. ${ }^{82}$ \\
\hline G624(2) & $\mathrm{CC}(\mathrm{O}) \mathrm{CCl}+\mathrm{OH} \rightarrow \mathrm{CC}(=\mathrm{O}) \mathrm{CCl}+\mathrm{HO}_{2}$ & $\mathrm{k}=2.60 \cdot 10^{-12} \exp (200 / \mathrm{T})$ & \\
\hline G625(2) & $\mathrm{CC}([\mathrm{O}]) \mathrm{CCl} \rightarrow \mathrm{CC}(=\mathrm{O}) \mathrm{CCl}+\mathrm{HO}_{2}$ & $\mathrm{k}=1.50 \cdot 10^{-14} \exp (-230 / \mathrm{T})$ & \\
\hline G626(2) & $\mathrm{CC}(=\mathrm{O}) \mathrm{C}(\mathrm{O}) \mathrm{Cl}+\mathrm{OH} \rightarrow \mathrm{CC}(=\mathrm{O}) \mathrm{C}(=\mathrm{O}) \mathrm{Cl}+\mathrm{HO}_{2}$ & $\mathrm{k}=3.00 \cdot 10^{-12}$ & \\
\hline G627(2) & $\mathrm{CC}(\mathrm{Cl})(\mathrm{Cl}) \mathrm{Cl}+\mathrm{Cl} \rightarrow \mathrm{ClC}(\mathrm{Cl})(\mathrm{Cl}) \mathrm{CO}[\mathrm{O}]+\mathrm{HCl}$ & $\mathrm{k}=2.80 \cdot 10^{-12} \exp (-1790 / \mathrm{T})$ & \\
\hline G628(2) & $\mathrm{ClC}(\mathrm{Cl}) \mathrm{Cl}+\mathrm{Cl} \rightarrow[\mathrm{O}] \mathrm{OC}(\mathrm{Cl})(\mathrm{Cl}) \mathrm{Cl}+\mathrm{HCl}$ & $\mathrm{k}=2.40 \cdot 10^{-12} \exp (-920 / \mathrm{T})$ & \\
\hline G629(2) & {$[\mathrm{O}] \mathrm{OC}(\mathrm{Cl})(\mathrm{Cl}) \mathrm{Cl}+\mathrm{NO}_{2} \rightarrow \mathrm{ClC}(\mathrm{Cl})(\mathrm{Cl}) \mathrm{OON}(=\mathrm{O})=\mathrm{O}$} & TROEF & \\
\hline G630(2) & $\mathrm{ClC}(\mathrm{Cl})(\mathrm{Cl}) \mathrm{OON}(=\mathrm{O})=\mathrm{O} \rightarrow[\mathrm{O}] \mathrm{OC}(\mathrm{Cl})(\mathrm{Cl}) \mathrm{Cl}+\mathrm{NO}_{2}$ & TROEXP & \\
\hline G631 (2) & $\mathrm{ClCCl}+\mathrm{Cl} \rightarrow[\mathrm{O}] \mathrm{OC}(\mathrm{Cl}) \mathrm{Cl}+\mathrm{HCl}$ & $\mathrm{k}=5.90 \cdot 10^{-12} \exp (-850 / \mathrm{T})$ & \\
\hline G632(2) & $\mathrm{O}=\mathrm{C}(\mathrm{Cl}) \mathrm{Cl}+\mathrm{OH} \rightarrow \mathrm{O}=[\mathrm{C}] \mathrm{Cl}+\mathrm{HOCl}$ & $\mathrm{k}=5.00 \cdot 10^{-15}$ & \\
\hline G633 (2) & $\mathrm{CCl}+\mathrm{Cl} \rightarrow \mathrm{ClCO}[\mathrm{O}]+\mathrm{HCl}$ & $\mathrm{k}=2.30 \cdot 10^{-11} \exp (-1150 / \mathrm{T})$ & \\
\hline G634(2) & $\mathrm{O}=\mathrm{CCl}+\mathrm{Cl} \rightarrow \mathrm{O}=[\mathrm{C}] \mathrm{Cl}+\mathrm{HCl}$ & $\mathrm{k}=8.10 \cdot 10^{-12} \exp (-710 / \mathrm{T})$ & \\
\hline G635(2) & $\mathrm{O}=[\mathrm{C}] \mathrm{Cl} \rightarrow \mathrm{Cl}+\mathrm{CO}$ & $\mathrm{k}=[\mathrm{M}]^{*} 4.10 \cdot 10^{-10} \exp (2960 / \mathrm{T})$ & \\
\hline G636(2) & $\mathrm{Cl}+\mathrm{CO} \rightarrow \mathrm{O}=[\mathrm{C}] \mathrm{Cl}$ & $\mathrm{k}=[\mathrm{M}]^{*} 1.30 \cdot 10^{-33}(\mathrm{~T} / 300)^{3.8}$ & \\
\hline G637(2) & $\mathrm{Br}+\mathrm{O}_{3} \rightarrow \mathrm{BrO}$ & $\mathrm{k}=1.70 \cdot 10^{-11} \exp (-800 / \mathrm{T})$ & \\
\hline G638(2) & $\mathrm{Br}+\mathrm{HO}_{2} \rightarrow \mathrm{HBr}$ & $\mathrm{k}=7.70 \cdot 10^{-12} \exp (-450 / \mathrm{T})$ & \\
\hline G639(2) & $\mathrm{Br}+\mathrm{H}_{2} \mathrm{O}_{2} \rightarrow \mathrm{HBr}+\mathrm{HO}_{2}$ & $\mathrm{k}=1.00 \cdot 10^{-11} \exp (-3000 / \mathrm{T})$ & \\
\hline G640 (2) & $\mathrm{Br}_{2}+\mathrm{OH} \rightarrow \mathrm{HOBr}+\mathrm{Br}$ & $\mathrm{k}=2.00 \cdot 10^{-11} \exp (-240 / \mathrm{T})$ & \\
\hline G641 (2) & $\mathrm{BrO}+\mathrm{O}_{3} \rightarrow 0.9 \mathrm{Br}+0.1 \mathrm{OBrO}$ & $\mathrm{k}=1.00 \cdot 10^{-12} \exp (-3200 / \mathrm{T})$ & \\
\hline G642(2) & $\mathrm{BrO}+\mathrm{OH} \rightarrow \mathrm{Br}+\mathrm{HO}_{2}$ & $\mathrm{k}=1.80 \cdot 10^{-11} \exp (-250 / \mathrm{T})$ & \\
\hline G643 (2) & $\mathrm{BrO}+\mathrm{HO}_{2} \rightarrow \mathrm{HOBr}$ & $\mathrm{k}=4.50 \cdot 10^{-12} \exp (-500 / \mathrm{T})$ & \\
\hline G644(2) & $\mathrm{BrO}+\mathrm{BrO} \rightarrow 1.7 \mathrm{Br}+0.15 \mathrm{Br}_{2}$ & $\mathrm{k}=1.60 \cdot 10^{-12} \exp (-210 / \mathrm{T})$ & \\
\hline G645 (2) & $\mathrm{HBr}+\mathrm{OH} \rightarrow \mathrm{Br}$ & $\mathrm{k}=6.70 \cdot 10^{-12} \exp (-155 / \mathrm{T})$ & \\
\hline G646(2) & $\mathrm{Br}+\mathrm{NO}_{2} \rightarrow \mathrm{BrNO}_{2}$ & TROEF & \\
\hline G647(2) & $\mathrm{Br}+\mathrm{NO}_{3} \rightarrow \mathrm{BrO}+\mathrm{NO}_{2}$ & $\mathrm{k}=1.60 \cdot 10^{-11}$ & \\
\hline G648(2) & $\mathrm{BrO}+\mathrm{NO} \rightarrow \mathrm{Br}+\mathrm{NO}_{2}$ & $\mathrm{k}=8.70 \cdot 10^{-12} \exp (-260 / \mathrm{T})$ & \\
\hline G649(2) & $\mathrm{BrO}+\mathrm{NO}_{2} \rightarrow \mathrm{BrNO}_{3}$ & TROEF & \\
\hline G650(2) & $\mathrm{BrNO}_{3} \rightarrow \mathrm{BrO}+\mathrm{NO}_{2}$ & $\mathrm{k}=2.79 \cdot 10^{13} \exp (-12360 / \mathrm{T})$ & \\
\hline G651 (2) & $\mathrm{Br}+\mathrm{BrNO}_{3} \rightarrow \mathrm{Br}_{2}+\mathrm{NO}_{3}$ & $\mathrm{k}=4.90 \cdot 10^{-11}$ & \\
\hline G652(2) & $\mathrm{HBr}+\mathrm{NO}_{3} \rightarrow \mathrm{Br}+\mathrm{HNO}_{3}$ & $\mathrm{k}=1.00 \cdot 10^{-16}$ & \\
\hline
\end{tabular}




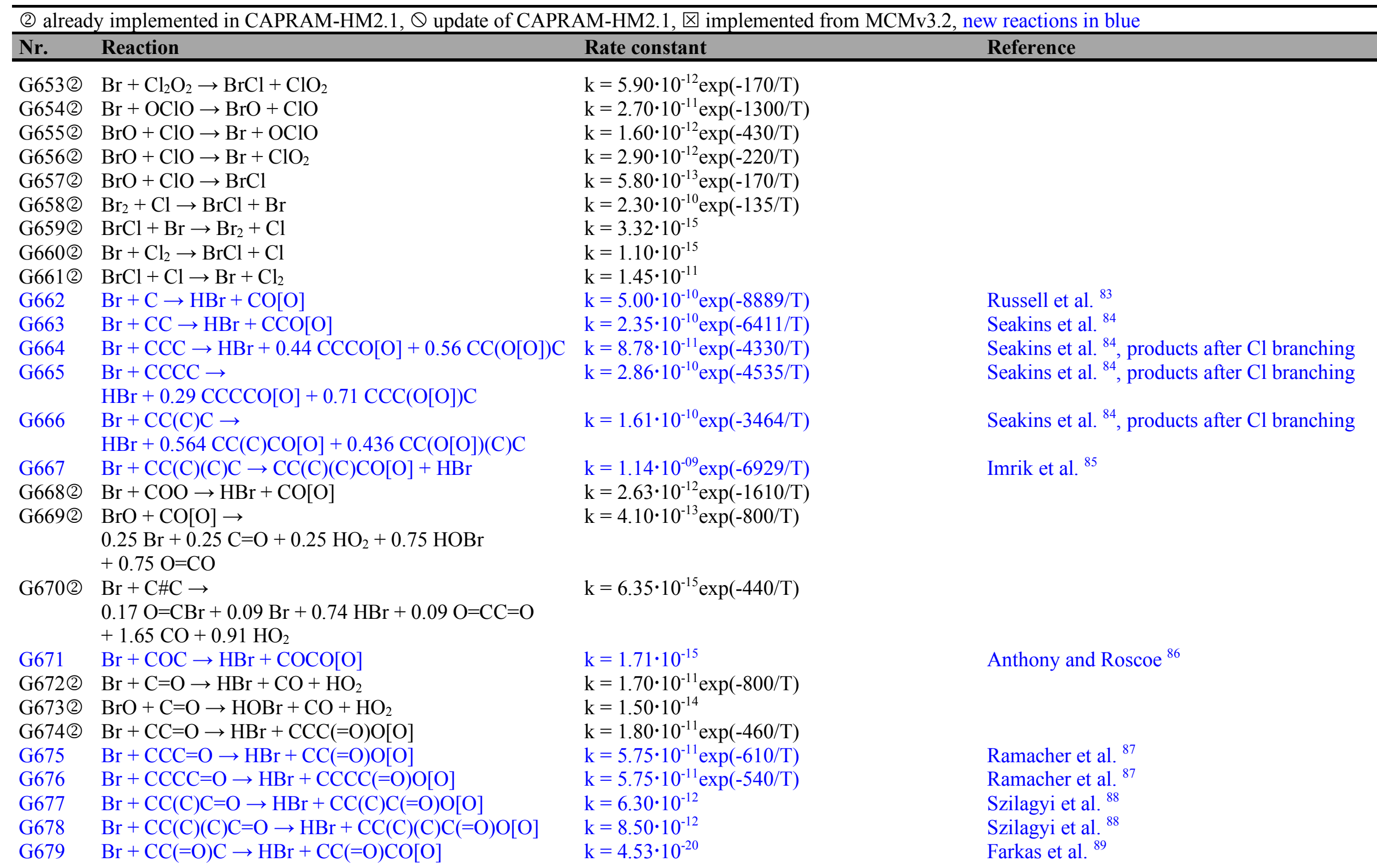




\begin{tabular}{|c|c|c|c|}
\hline Nr. & Reaction & Rate constant & Reference \\
\hline G680 & $\mathrm{Br}+\mathrm{O}=\mathrm{CC}=\mathrm{O} \rightarrow \mathrm{HBr}+\mathrm{O}=\mathrm{CC}(=\mathrm{O}) \mathrm{O}[\mathrm{O}]$ & $\mathrm{k}=1.40 \cdot 10^{-14}$ & Ramacher et al. ${ }^{90}$ \\
\hline G681 & $\begin{array}{l}\mathrm{Br}+\mathrm{O}=\mathrm{CC}=\mathrm{C} \rightarrow \\
0.2 \mathrm{HBr}+0.2 \mathrm{C}=\mathrm{CC}(=\mathrm{O}) \mathrm{O}[\mathrm{O}]+0.8 \mathrm{BrCC}(\mathrm{O}[\mathrm{O}]) \mathrm{C}=\mathrm{O}\end{array}$ & $\mathrm{k}=3.21 \cdot 10^{-12}$ & Sauer et al. ${ }^{91}$ \\
\hline G682 & $\begin{array}{l}\mathrm{BrCC}(\mathrm{O}[\mathrm{O}]) \mathrm{C}=\mathrm{O}+\mathrm{NO} \rightarrow \\
\mathrm{BrCC}=\mathrm{O}+\mathrm{HO}_{2}+\mathrm{CO}+\mathrm{NO}_{2}\end{array}$ & $\mathrm{k}=2.70 \cdot 10^{-12} \exp (360 / \mathrm{T})$ & Rate constant MCM, products Toyota et al. ${ }^{52}$ \\
\hline G683 & $\begin{array}{l}\mathrm{BrCC}(\mathrm{O}[\mathrm{O}]) \mathrm{C}=\mathrm{O}+\mathrm{NO}_{3} \rightarrow \\
\mathrm{BrCC}=\mathrm{O}+\mathrm{HO}_{2}+\mathrm{CO}+\mathrm{NO}_{2}\end{array}$ & $\mathrm{k}=2.30 \cdot 10^{-12}$ & Rate constant MCM, products Toyota et al. ${ }^{52}$ \\
\hline G684 & $\mathrm{BrCC}(\mathrm{O}[\mathrm{O}]) \mathrm{C}=\mathrm{O} \rightarrow \mathrm{BrCC}=\mathrm{O}+\mathrm{HO}_{2}+\mathrm{CO}$ & $\mathrm{k}=8.80 \cdot 10^{-13} * \mathrm{RO}_{2}$ & Rate constant MCM, products Toyota et al. ${ }^{52}$ \\
\hline $\mathrm{G} 6850$ & $\mathrm{Br}+\operatorname{clcccc} 1 \mathrm{C} \rightarrow \mathrm{HBr}+\operatorname{clcccc} 1 \mathrm{CO}[\mathrm{O}]$ & $\mathrm{k}=3.70 \cdot 10^{-12} \exp (-1630 / \mathrm{T})$ & Giri and Roscoe 92 \\
\hline G686 & $\mathrm{Br}+\mathrm{Cc} 1 \mathrm{ccccc} 1 \mathrm{C} \rightarrow \mathrm{HBr}+\mathrm{Cc} 1 \operatorname{ccccc} 1 \mathrm{CO}[\mathrm{O}]$ & $\mathrm{k}=8.90 \cdot 10^{-14}$ & Bierbach et al. ${ }^{93}$ \\
\hline G687 & $\mathrm{Br}+\mathrm{c} 1 \mathrm{c}(\mathrm{C}) \mathrm{cccc} 1 \mathrm{C} \rightarrow \mathrm{Cc} 1 \mathrm{cccc}(\mathrm{c} 1) \mathrm{CO}[\mathrm{O}]+\mathrm{HBr}$ & $\mathrm{k}=6.60 \cdot 10^{-14}$ & Bierbach et al. ${ }^{93}$ \\
\hline G688 & $\mathrm{Br}+\mathrm{c} 1 \mathrm{cc}(\mathrm{C}) \operatorname{ccc} 1 \mathrm{C} \rightarrow \mathrm{Cc} 1 \mathrm{ccc}(\mathrm{CO}[\mathrm{O}]) \mathrm{cc} 1+\mathrm{HBr}$ & $\mathrm{k}=9.00 \cdot 10^{-14}$ & Bierbach et al. ${ }^{93}$ \\
\hline G689 & $\mathrm{Br}+\mathrm{c} 1 \mathrm{c}(\mathrm{C}) \mathrm{cc}(\mathrm{C}) \mathrm{cc} 1 \mathrm{C} \rightarrow \mathrm{c} 1 \mathrm{c}(\mathrm{C}) \mathrm{cc}(\mathrm{CO}[\mathrm{O}]) \mathrm{cc} 1 \mathrm{C}+\mathrm{HBr}$ & $\mathrm{k}=4.80 \cdot 10^{-13}$ & Bierbach et al. ${ }^{93}$ \\
\hline G690 & $\mathrm{Br}+\mathrm{OC} \rightarrow \mathrm{HBr}+\mathrm{C}=\mathrm{O}+\mathrm{HO}_{2}$ & $\mathrm{k}=5.00 \cdot 10^{-16}$ & Bierbach et al. ${ }^{93}$ \\
\hline G691 & $\begin{array}{l}\mathrm{Br}+\mathrm{CCO} \rightarrow \\
0.95 \mathrm{CC}=\mathrm{O}+0.95 \mathrm{HO}_{2}+0.05 \mathrm{OCCO}[\mathrm{O}]+\mathrm{HBr}\end{array}$ & $\mathrm{k}=9.80 \cdot 10^{-15}$ & Bierbach et al. ${ }^{93}$ \\
\hline G692 & $\begin{array}{l}\mathrm{Br}+\mathrm{CCCO} \rightarrow \\
0.69 \mathrm{CCC}=\mathrm{O}+0.69 \mathrm{HO}_{2}+0.06 \mathrm{OCCCO}[\mathrm{O}] \\
+0.25 \mathrm{CC}(\mathrm{O}[\mathrm{O}]) \mathrm{CO}+\mathrm{HBr}\end{array}$ & $\mathrm{k}=8.30 \cdot 10^{-15}$ & Bierbach et al. ${ }^{93}$ \\
\hline G693 & $\mathrm{Br}+\mathrm{CC}(\mathrm{O}) \mathrm{C} \rightarrow \mathrm{CC}(=\mathrm{O}) \mathrm{C}+\mathrm{HBr}$ & $\mathrm{k}=4.60 \cdot 10^{-14}$ & Bierbach et al. ${ }^{93}$ \\
\hline G694 & $\begin{array}{l}\mathrm{Br}+\mathrm{CC}(\mathrm{O})(\mathrm{C}) \mathrm{C} \rightarrow \\
\mathrm{HBr}+0.888 \mathrm{CC}(\mathrm{O})(\mathrm{C}) \mathrm{CO}[\mathrm{O}]+0.112 \mathrm{CC}([\mathrm{O}])(\mathrm{C}) \mathrm{C}\end{array}$ & $\mathrm{k}=5.00 \cdot 10^{-16}$ & Bierbach et al. ${ }^{93}$ \\
\hline G695(2) & $\mathrm{Br}+\mathrm{C}=\mathrm{C} \rightarrow \mathrm{BrCCO}[\mathrm{O}]$ & $\mathrm{k}=2.25 \cdot 10^{-13} \exp (-277 / \mathrm{T})$ & \\
\hline G696(2) & $\begin{array}{l}\mathrm{BrCCO}[\mathrm{O}] \rightarrow \\
0.2 \mathrm{OCCBr}+0.2 \mathrm{BrCC}=\mathrm{O}+0.6 \mathrm{BrCC}[\mathrm{O}]\end{array}$ & $\mathrm{k}=2.00 \cdot 10^{-12} * \mathrm{RO}_{2}$ & \\
\hline G697(2) & $\mathrm{BrCCO}[\mathrm{O}]+\mathrm{NO} \rightarrow \mathrm{BrCC}[\mathrm{O}]+\mathrm{NO}_{2}$ & $\mathrm{k}=9.70 \cdot 10^{-12}$ & \\
\hline G698(2) & $\mathrm{OCCBr}+\mathrm{OH} \rightarrow \mathrm{BrCC}=\mathrm{O}+\mathrm{HO}_{2}$ & $\mathrm{k}=4.60 \cdot 10^{-12}$ & \\
\hline G699(2) & $\mathrm{BrCC}[\mathrm{O}] \rightarrow \mathrm{BrCC}=\mathrm{O}+\mathrm{HO}_{2}$ & $\mathrm{k}=6.00 \cdot 10^{-14} \exp (-550 / \mathrm{T})$ & \\
\hline G7002) & $\mathrm{Br}+\mathrm{CC}=\mathrm{C} \rightarrow \mathrm{CC}(\mathrm{O}[\mathrm{O}]) \mathrm{CBr}$ & $\mathrm{k}=3.60 \cdot 10^{-12}$ & \\
\hline G701 (2) & $\begin{array}{l}\mathrm{CC}(\mathrm{O}[\mathrm{O}]) \mathrm{CBr} \rightarrow \\
0.2 \mathrm{CC}(\mathrm{O}) \mathrm{CBr}+0.2 \mathrm{CC}(=\mathrm{O}) \mathrm{CBr}+0.6 \mathrm{CC}([\mathrm{O}]) \mathrm{CBr}\end{array}$ & $\mathrm{k}=4.00 \cdot 10^{-14} * \mathrm{RO}_{2}$ & \\
\hline $\begin{array}{l}\mathrm{G} 702(2) \\
\mathrm{G} 703(2)\end{array}$ & $\begin{array}{l}\mathrm{CC}(\mathrm{O}[\mathrm{O}]) \mathrm{CBr}+\mathrm{NO} \rightarrow \mathrm{CC}([\mathrm{O}]) \mathrm{CBr}+\mathrm{NO}_{2} \\
\mathrm{CC}(\mathrm{O}) \mathrm{CBr}+\mathrm{OH} \rightarrow \mathrm{CC}(=\mathrm{O}) \mathrm{CBr}+\mathrm{HO}_{2}\end{array}$ & $\begin{array}{l}\mathrm{k}=2.70 \cdot 10^{-12} \exp (360 / \mathrm{T}) \\
\mathrm{k}=2.60 \cdot 10^{-12} \exp (200 / \mathrm{T})\end{array}$ & \\
\hline
\end{tabular}




\begin{tabular}{|c|c|c|c|}
\hline Nr. & Reaction & Rate constant & Reference \\
\hline G704(2) & $\mathrm{CC}([\mathrm{O}]) \mathrm{CBr} \rightarrow \mathrm{CC}(=\mathrm{O}) \mathrm{CBr}+\mathrm{HO}_{2}$ & $\mathrm{k}=1.50 \cdot 10^{-14} \exp (-230 / \mathrm{T})$ & \\
\hline G705(2) & $\mathrm{CC}(=\mathrm{O}) \mathrm{CBr}+\mathrm{OH} \rightarrow \mathrm{CC}(=\mathrm{O}) \mathrm{C}(\mathrm{Br}) \mathrm{O}[\mathrm{O}]$ & $\mathrm{k}=8.80 \cdot 10^{-12} \exp (-1320 / \mathrm{T})$ & \\
\hline \multirow[t]{3}{*}{ G706(2) } & $\mathrm{CC}(=\mathrm{O}) \mathrm{C}(\mathrm{Br}) \mathrm{O}[\mathrm{O}] \rightarrow$ & $\mathrm{k}=2.00 \cdot 10^{-12} * \mathrm{RO}_{2}$ & \\
\hline & $0.2 \mathrm{CC}(=\mathrm{O}) \mathrm{C}(\mathrm{O}) \mathrm{Br}+0.2 \mathrm{CC}(=\mathrm{O}) \mathrm{C}(=\mathrm{O}) \mathrm{Br}$ & & \\
\hline & $+0.6 \mathrm{CC}(=\mathrm{O}) \mathrm{O}[\mathrm{O}]+0.6 \mathrm{O}=\mathrm{CBr}$ & & \\
\hline \multirow[t]{2}{*}{ G707(2) } & $\mathrm{CC}(=\mathrm{O}) \mathrm{C}(\mathrm{Br}) \mathrm{O}[\mathrm{O}]+\mathrm{NO} \rightarrow$ & $\mathrm{k}=8.00 \cdot 10^{-12}$ & \\
\hline & $\mathrm{CC}(=\mathrm{O}) \mathrm{O}[\mathrm{O}]+\mathrm{O}=\mathrm{CBr}+\mathrm{NO}_{2}$ & & \\
\hline G708(2) & $\mathrm{CC}(=\mathrm{O}) \mathrm{C}(\mathrm{O}) \mathrm{Br}+\mathrm{OH} \rightarrow \mathrm{CC}(=\mathrm{O}) \mathrm{C}(=\mathrm{O}) \mathrm{Br}+\mathrm{HO}_{2}$ & $\mathrm{k}=3.00 \cdot 10^{-12}$ & \\
\hline G709(2) & $\mathrm{BrC}(\mathrm{Br}) \mathrm{Br}+\mathrm{OH} \rightarrow \mathrm{BrC}(\mathrm{Br})(\mathrm{Br}) \mathrm{O}[\mathrm{O}]$ & $\mathrm{k}=1.35 \cdot 10^{-12} \exp (-600 / \mathrm{T})$ & \\
\hline G710(2) & $\mathrm{BrC}(\mathrm{Br}) \mathrm{Br}+\mathrm{Cl} \rightarrow \mathrm{BrC}(\mathrm{Br})(\mathrm{Br}) \mathrm{O}[\mathrm{O}]+\mathrm{HCl}$ & $\mathrm{k}=4.85 \cdot 10^{-12} \exp (-850 / \mathrm{T})$ & \\
\hline G711(2) & $\mathrm{BrC}(\mathrm{Br})(\mathrm{Br}) \mathrm{O}[\mathrm{O}]+\mathrm{HO}_{2} \rightarrow \mathrm{O}=\mathrm{C}(\mathrm{Br}) \mathrm{Br}+\mathrm{HOBr}$ & $\mathrm{k}=4.70 \cdot 10^{-13} \exp (710 / \mathrm{T})$ & \\
\hline \multirow[t]{2}{*}{ G712(2) } & $\mathrm{BrC}(\mathrm{Br})(\mathrm{Br}) \mathrm{O}[\mathrm{O}] \rightarrow$ & $\mathrm{k}=6.60 \cdot 10^{-12 *} \mathrm{RO}_{2}$ & \\
\hline & $0.3 \mathrm{OC}(\mathrm{Br})(\mathrm{Br}) \mathrm{Br}+0.7 \mathrm{BrC}(\mathrm{Br})(\mathrm{Br})[\mathrm{O}]$ & & \\
\hline G713(2) & $\mathrm{BrC}(\mathrm{Br})(\mathrm{Br}) \mathrm{O}[\mathrm{O}]+\mathrm{NO} \rightarrow \mathrm{O}=\mathrm{C}(\mathrm{Br}) \mathrm{Br}+\mathrm{Br}+\mathrm{NO}_{2}$ & $\mathrm{k}=7.30 \cdot 10^{-12} \exp (270 / \mathrm{T})$ & \\
\hline G714(2) & $\mathrm{BrC}(\mathrm{Br})(\mathrm{Br}) \mathrm{O}[\mathrm{O}]+\mathrm{NO}_{2} \rightarrow \mathrm{BrC}(\mathrm{Br})(\mathrm{Br}) \mathrm{OON}(=\mathrm{O})=\mathrm{O}$ & TROEF & \\
\hline G715(2) & $\mathrm{BrC}(\mathrm{Br})(\mathrm{Br}) \mathrm{OON}(=\mathrm{O})=\mathrm{O} \rightarrow \mathrm{BrC}(\mathrm{Br})(\mathrm{Br}) \mathrm{O}[\mathrm{O}]+\mathrm{NO}_{2}$ & TROEXP & \\
\hline G716(2) & $\mathrm{OC}(\mathrm{Br})(\mathrm{Br}) \mathrm{Br}+\mathrm{OH} \rightarrow \mathrm{BrC}(\mathrm{Br})(\mathrm{Br})[\mathrm{O}]$ & $\mathrm{k}=3.60 \cdot 10^{-14}$ & \\
\hline G717(2) & $\mathrm{BrC}(\mathrm{Br})(\mathrm{Br})[\mathrm{O}] \rightarrow \mathrm{O}=\mathrm{C}(\mathrm{Br}) \mathrm{Br}+\mathrm{Br}$ & $\mathrm{k}=4.00 \cdot 10^{13} \exp (-4600 / \mathrm{T})$ & \\
\hline G718(2) & $\mathrm{BrCBr}+\mathrm{OH} \rightarrow \mathrm{BrC}(\mathrm{Br}) \mathrm{O}[\mathrm{O}]$ & $\mathrm{k}=1.50 \cdot 10^{-12} \exp (-775 / \mathrm{T})$ & \\
\hline G719(2) & $\mathrm{BrCBr}+\mathrm{Cl} \rightarrow \mathrm{BrC}(\mathrm{Br}) \mathrm{O}[\mathrm{O}]+\mathrm{HCl}$ & $\mathrm{k}=6.30 \cdot 10^{-12} \exp (-800 / \mathrm{T})$ & \\
\hline \multirow[t]{2}{*}{ G720(2) } & $\mathrm{BrC}(\mathrm{Br}) \mathrm{O}[\mathrm{O}]+\mathrm{HO}_{2} \rightarrow$ & $\mathrm{k}=5.60 \cdot 10^{-13} \exp (700 / \mathrm{T})$ & \\
\hline & $0.3 \mathrm{O}=\mathrm{CBr}+0.3 \mathrm{HOBr}+0.7 \mathrm{O}=\mathrm{C}(\mathrm{Br}) \mathrm{Br}$ & & \\
\hline \multirow[t]{2}{*}{ G721(2) } & $\mathrm{BrC}(\mathrm{Br}) \mathrm{O}[\mathrm{O}] \rightarrow$ & $\mathrm{k}=2.00 \cdot 10^{-12} * \mathrm{RO}_{2}$ & \\
\hline & $\begin{array}{l}0.2 \mathrm{O}=\mathrm{C}(\mathrm{Br}) \mathrm{Br}+0.2 \mathrm{OC}(\mathrm{Br}) \mathrm{Br}+0.6 \mathrm{O}=\mathrm{CBr}+0.6 \mathrm{Br} \\
+0.6 \mathrm{HO}_{2}\end{array}$ & & \\
\hline G722(2) & $\mathrm{BrC}(\mathrm{Br}) \mathrm{O}[\mathrm{O}]+\mathrm{NO} \rightarrow \mathrm{O}=\mathrm{CBr}+\mathrm{Br}+\mathrm{NO}_{2}$ & $\mathrm{k}=1.70 \cdot 10^{-11}$ & \\
\hline G723(2) & $\mathrm{OC}(\mathrm{Br}) \mathrm{Br}+\mathrm{OH} \rightarrow \mathrm{O}=\mathrm{C}(\mathrm{Br}) \mathrm{Br}+\mathrm{HO}_{2}$ & $\mathrm{k}=9.34 \cdot 10^{-13}$ & \\
\hline G724(2) & $\mathrm{O}=\mathrm{C}(\mathrm{Br}) \mathrm{Br}+\mathrm{OH} \rightarrow \mathrm{O}=[\mathrm{C}] \mathrm{Br}+\mathrm{HOBr}$ & $\mathrm{k}=5.00 \cdot 10^{-15}$ & \\
\hline G725(2) & $\mathrm{CBr}+\mathrm{OH} \rightarrow \mathrm{BrCO}[\mathrm{O}]+\mathrm{H}_{2} \mathrm{O}$ & $\mathrm{k}=7.40 \cdot 10^{-13} \exp (-875 / \mathrm{T})$ & implemented from MCM \\
\hline G726(2) & $\mathrm{CBr}+\mathrm{Cl} \rightarrow \mathrm{BrCO}[\mathrm{O}]+\mathrm{HCl}$ & $\mathrm{k}=1.40 \cdot 10^{-11} \exp (-1030 / \mathrm{T})$ & \\
\hline G727(2) & $\mathrm{BrCO}[\mathrm{O}]+\mathrm{HO}_{2} \rightarrow \mathrm{BrCOO}$ & $\mathrm{k}=4.28 \cdot 10^{-13} \exp (820 / \mathrm{T})$ & \\
\hline G728(2) & $\mathrm{BrCO}[\mathrm{O}]+\mathrm{NO} \rightarrow \mathrm{BrC}[\mathrm{O}]+\mathrm{NO}_{2}$ & $\mathrm{k}=4.05 \cdot 10^{-12} \exp (360 / \mathrm{T})$ & \\
\hline G729凶 & $\mathrm{BrCO}[\mathrm{O}]+\mathrm{NO}_{3} \rightarrow \mathrm{BrC}[\mathrm{O}]+\mathrm{NO}_{2}$ & $\mathrm{k}=2.30 \cdot 10^{-12}$ & \\
\hline
\end{tabular}




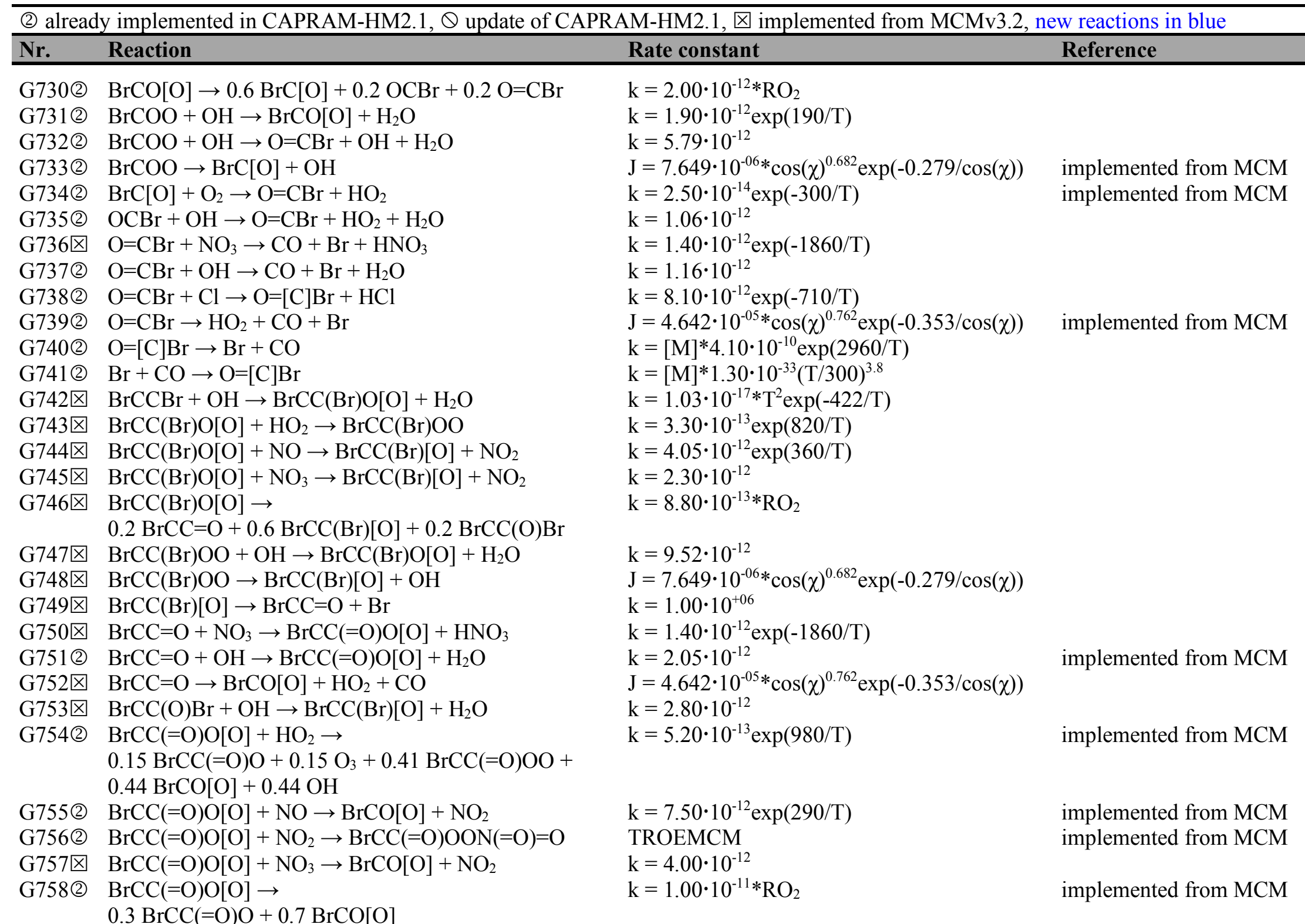




\begin{tabular}{|c|c|c|}
\hline \multicolumn{3}{|c|}{ (2) already implemented in CAPRAM-HM2.1, $Q$ update of CAPRAM-HM2.1, $囚$ implemented } \\
\hline Nr. & Reaction & Rate constant \\
\hline $\mathrm{G} 759$ (2) & $\mathrm{BrCC}(=\mathrm{O}) \mathrm{O}+\mathrm{OH} \rightarrow \mathrm{BrCO}[\mathrm{O}]+\mathrm{H}_{2} \mathrm{O}$ & $\mathrm{k}=1.90 \cdot 10^{-12} \exp (190 / \mathrm{T})$ \\
\hline G760(2) & $\mathrm{BrCC}(=\mathrm{O}) \mathrm{OO}+\mathrm{OH} \rightarrow \mathrm{BrCC}(=\mathrm{O}) \mathrm{O}[\mathrm{O}]+\mathrm{H}_{2} \mathrm{O}$ & $\mathrm{k}=3.79 \cdot 10^{-12}$ \\
\hline G761 (2) & $\mathrm{BrCC}(=\mathrm{O}) \mathrm{OO} \rightarrow \mathrm{BrCO}[\mathrm{O}]+\mathrm{OH}$ & $\mathrm{J}=7.649 \cdot 10^{-06 *} \cos (\chi)^{0.682} \mathrm{ex}$ \\
\hline G762(2) & $\begin{array}{l}\mathrm{BrCC}(=\mathrm{O}) \mathrm{OON}(=\mathrm{O})=\mathrm{O}+\mathrm{OH} \rightarrow \\
\mathrm{O}=\mathrm{CBr}+\mathrm{CO}+\mathrm{NO}_{2}\end{array}$ & $\mathrm{k}=5.56 \cdot 10^{-13}$ \\
\hline G763(2) & $\begin{array}{l}\mathrm{BrCC}(=\mathrm{O}) \mathrm{OON}(=\mathrm{O})=\mathrm{O} \rightarrow \\
\mathrm{BrCC}(=\mathrm{O}) \mathrm{O}[\mathrm{O}]+\mathrm{NO}_{2}\end{array}$ & TROEMCM \\
\hline G764(2) & $\mathrm{I}+\mathrm{I} \rightarrow \mathrm{I}_{2}$ & $\mathrm{k}=2.99 \cdot 10^{-11}$ \\
\hline G765(2) & $\mathrm{I}+\mathrm{O}_{3} \rightarrow \mathrm{IO}$ & $\mathrm{k}=2.10 \cdot 10^{-11} \exp (-830 / \mathrm{T})$ \\
\hline G766(2) & $\mathrm{I}_{2}+\mathrm{OH} \rightarrow \mathrm{I}+\mathrm{HOI}$ & $\mathrm{k}=2.10 \cdot 10^{-10}$ \\
\hline G767(2) & $\mathrm{I}+\mathrm{HO}_{2} \rightarrow \mathrm{HI}$ & $\mathrm{k}=1.50 \cdot 10^{-11} \exp (-1090 / \mathrm{T})$ \\
\hline G768(2) & $\mathrm{IO}+\mathrm{HO}_{2} \rightarrow \mathrm{HOI}$ & $\mathrm{k}=1.40 \cdot 10^{-11} \exp (540 / \mathrm{T})$ \\
\hline G7690 & $\mathrm{IO}+\mathrm{IO} \rightarrow 0.38 \mathrm{OIO}+0.46 \mathrm{I}_{2} \mathrm{O}_{2}+0.6 \mathrm{I}+0.05 \mathrm{I}_{2}$ & $\mathrm{k}=5.40 \cdot 10^{-11} \exp (180 / \mathrm{T})$ \\
\hline G770@ & $\mathrm{OIO}+\mathrm{OH} \rightarrow \mathrm{HIO}_{3}$ & $\mathrm{k}=2.20 \cdot 10^{-10} \exp (243 / \mathrm{T})$ \\
\hline G771 & $\mathrm{IO}+\mathrm{O}_{3} \rightarrow 0.83 \mathrm{I}+0.17 \mathrm{OIO}$ & $\mathrm{k}=1.20 \cdot 10^{-15}$ \\
\hline G772 & $\mathrm{IO}+\mathrm{OIO} \rightarrow \mathrm{I}_{2} \mathrm{O}_{3}$ & $\mathrm{k}=1.00 \cdot 10^{-10}$ \\
\hline G773 & $\mathrm{I}_{2} \mathrm{O}_{3} \rightarrow \mathrm{IO}+\mathrm{OIO}$ & $\mathrm{k}=2.78 \cdot 10^{-11}$ \\
\hline G774 & $\mathrm{OIO}+\mathrm{OIO} \rightarrow \mathrm{I}_{2} \mathrm{O}_{4}$ & $\mathrm{k}=1.00 \cdot 10^{-10}$ \\
\hline G775 & $\mathrm{I}_{2} \mathrm{O}_{4} \rightarrow \mathrm{OIO}+\mathrm{OIO}$ & $\mathrm{k}=1.67 \cdot 10^{+00}$ \\
\hline G776 & $\mathrm{I}_{2}+\mathrm{O}_{3} \rightarrow \mathrm{IO}+\mathrm{I}$ & $\mathrm{k}=4.02 \cdot 10^{-15} \exp (-2050 / \mathrm{T})$ \\
\hline G777@ & $\mathrm{I}_{2} \mathrm{O}_{2} \rightarrow 0.995 \mathrm{OIO}+0.995 \mathrm{I}+0.01 \mathrm{IO}$ & $\mathrm{k}=1.00 \cdot 10^{+01}$ \\
\hline G778(2) & $\mathrm{HI}+\mathrm{OH} \rightarrow \mathrm{I}+\mathrm{H}_{2} \mathrm{O}$ & $\mathrm{k}=1.60 \cdot 10^{-11} \exp (440 / \mathrm{T})$ \\
\hline G779(2) & $\mathrm{I}+\mathrm{NO} \rightarrow \mathrm{INO}$ & TROE \\
\hline G780(2) & $\mathrm{I}+\mathrm{NO}_{2} \rightarrow \mathrm{INO}_{2}$ & TROEF \\
\hline G781(2) & $\mathrm{I}+\mathrm{NO}_{3} \rightarrow \mathrm{IO}+\mathrm{NO}_{2}$ & $\mathrm{k}=4.50 \cdot 10^{-10}$ \\
\hline G782(2) & $\mathrm{I}_{2}+\mathrm{NO}_{3} \rightarrow \mathrm{I}+\mathrm{INO}_{3}$ & $\mathrm{k}=1.50 \cdot 10^{-12}$ \\
\hline G783(2) & $\mathrm{IO}+\mathrm{NO} \rightarrow \mathrm{I}+\mathrm{NO}_{2}$ & $\mathrm{k}=7.15 \cdot 10^{-12} \exp (300 / \mathrm{T})$ \\
\hline G784(2) & $\mathrm{IO}+\mathrm{NO}_{2} \rightarrow \mathrm{INO}_{3}$ & TROEF \\
\hline G785(2) & $\mathrm{OIO}+\mathrm{NO} \rightarrow \mathrm{IO}+\mathrm{NO}_{2}$ & $\mathrm{k}=1.10 \cdot 10^{-12} \exp (542 / \mathrm{T})$ \\
\hline G786(2) & $\mathrm{HI}+\mathrm{NO}_{3} \rightarrow \mathrm{I}+\mathrm{HNO}_{3}$ & $\mathrm{k}=1.30 \cdot 10^{-12} \exp (-1830 / \mathrm{T})$ \\
\hline G787(2) & $\mathrm{INO}+\mathrm{INO} \rightarrow \mathrm{I}_{2}+2.0 \mathrm{NO}$ & $\mathrm{k}=8.40 \cdot 10^{-11} \exp (-2620 / \mathrm{T})$ \\
\hline G788(2) & $\mathrm{INO}_{2}+\mathrm{INO}_{2} \rightarrow \mathrm{I}_{2}+2.0 \mathrm{NO}_{2}$ & $\mathrm{k}=4.70 \cdot 10^{-13} \exp (-1670 / \mathrm{T})$ \\
\hline
\end{tabular}




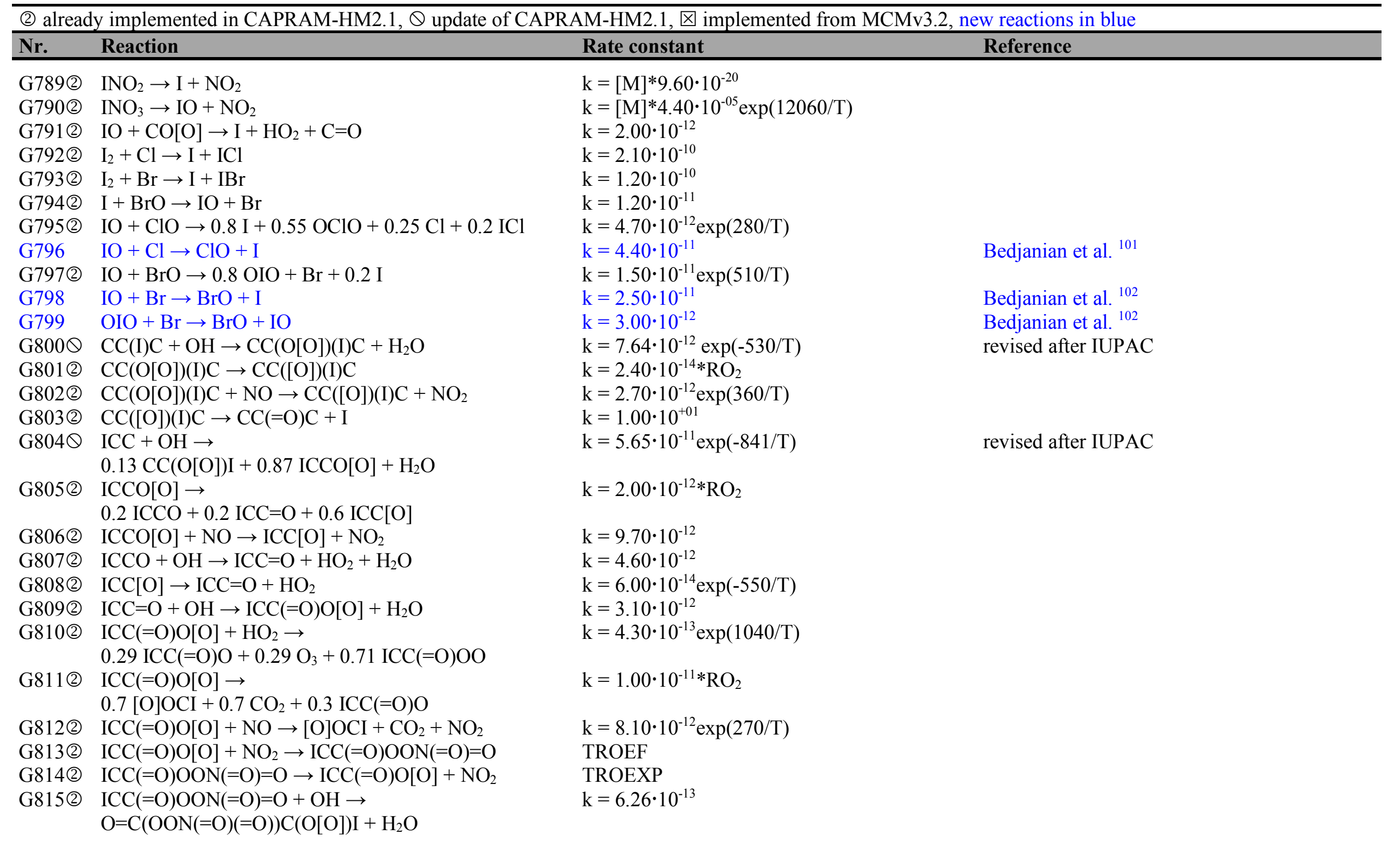




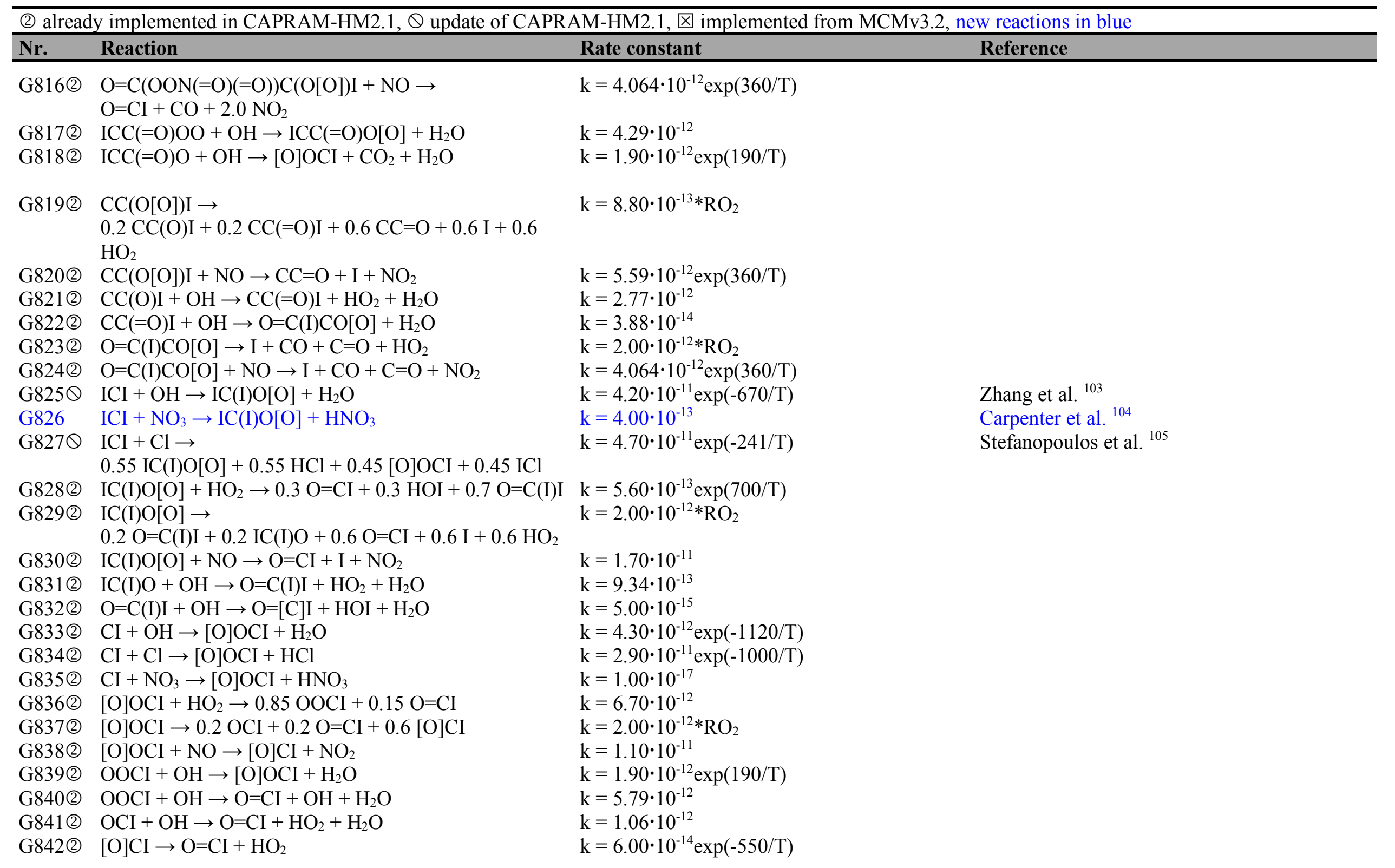


(2) already implemented in CAPRAM-HM2.1, $Q$ update of CAPRAM-HM2.1, $囚$ implemented from MCMv3.2, new reactions in blue

Nr. Reaction $\quad$ Rate constant

$\mathrm{G} 843(2) \mathrm{O}=\mathrm{CI}+\mathrm{OH} \rightarrow \mathrm{I}+\mathrm{CO}+\mathrm{H}_{2} \mathrm{O}$

$\mathrm{G} 844(2) \mathrm{O}=\mathrm{CI}+\mathrm{Cl} \rightarrow \mathrm{O}=[\mathrm{C}] \mathrm{I}+\mathrm{HCl}$

$\mathrm{k}=1.16 \cdot 10^{-12}$

$\mathrm{k}=8.10 \cdot 10^{-12} \exp (-710 / \mathrm{T})$

$\mathrm{G} 845$ (2) $\mathrm{O}=[\mathrm{C}] \mathrm{I} \rightarrow \mathrm{I}+\mathrm{CO}$

$\mathrm{k}=8.10 \cdot 10^{-12} \exp (-710 / \mathrm{T})$

$\mathrm{G} 846(2) \mathrm{I}+\mathrm{CO} \rightarrow \mathrm{O}=[\mathrm{C}] \mathrm{I}$

$\exp (2960 / \mathrm{T})$

Reference 
Table S5 Parameters for pressure dependent reactions.

\begin{tabular}{|c|c|c|c|c|c|}
\hline & Reaction & TYPE & $\mathbf{k}_{\mathbf{0}}$ & $\mathbf{k} \infty$ & $\mathbf{F}_{\mathbf{C}}$ \\
\hline G307 & $\mathrm{ClC}(\mathrm{Cl})(\mathrm{Cl}) \mathrm{C}(=\mathrm{O}) \mathrm{O}[\mathrm{O}]+\mathrm{NO}_{2} \rightarrow \mathrm{O}=\mathrm{N}(=\mathrm{O}) \mathrm{OOC}(=\mathrm{O}) \mathrm{C}(\mathrm{Cl})(\mathrm{Cl}) \mathrm{Cl}$ & TROEMCM & $2.70 \cdot 10^{-28 *}(\mathrm{~T} / 298)^{7.1}$ & $1.20 \cdot 10^{-11} *(\mathrm{~T} / 298)^{0.9}$ & 0.3 \\
\hline G313 & $\mathrm{ClCC}(=\mathrm{O}) \mathrm{O}[\mathrm{O}]+\mathrm{NO}_{2} \rightarrow \mathrm{ClCC}(=\mathrm{O}) \mathrm{OON}(=\mathrm{O})=\mathrm{O}$ & TROEMCM & $2.70 \cdot 10^{-28 *(T / 298)^{7.1}}$ & $1.20 \cdot 10^{-11 *(\mathrm{~T} / 298)^{0.9}}$ & 0.3 \\
\hline G318 & $\mathrm{OC}(\mathrm{Cl})(\mathrm{Cl}) \mathrm{C}(=\mathrm{O}) \mathrm{O}[\mathrm{O}]+\mathrm{NO}_{2} \rightarrow \mathrm{O}=\mathrm{N}(=\mathrm{O}) \mathrm{OOC}(=\mathrm{O}) \mathrm{C}(\mathrm{O})(\mathrm{Cl}) \mathrm{Cl}$ & TROEMCM & $2.70 \cdot 10^{-28 *(T / 298)^{7.1}}$ & $1.20 \cdot 10^{-11 *(\mathrm{~T} / 298)^{0.9}}$ & 0.3 \\
\hline G323 & $\mathrm{ClCC}(\mathrm{Cl}) \mathrm{C}(=\mathrm{O}) \mathrm{O}[\mathrm{O}]+\mathrm{NO}_{2} \rightarrow \mathrm{ClCC}(\mathrm{Cl}) \mathrm{C}(=\mathrm{O}) \mathrm{OON}(=\mathrm{O})=\mathrm{O}$ & TROEMCM & $2.70 \cdot 10^{-28 *(T / 298)^{7.1}}$ & $1.20 \cdot 10^{-11 *(\mathrm{~T} / 298)^{0.9}}$ & 0.3 \\
\hline G334 & $\mathrm{ClC}(\mathrm{Cl}) \mathrm{C}(=\mathrm{O}) \mathrm{O}[\mathrm{O}]+\mathrm{NO}_{2} \rightarrow \mathrm{O}=\mathrm{N}(=\mathrm{O}) \mathrm{OOC}(=\mathrm{O}) \mathrm{C}(\mathrm{Cl}) \mathrm{Cl}$ & TROEMCM & $2.70 \cdot 10^{-28 *(T / 298)^{7.1}}$ & $1.20 \cdot 10^{-11 *(\mathrm{~T} / 298)^{0.9}}$ & 0.3 \\
\hline G353 & $\mathrm{OC}(\mathrm{Cl}) \mathrm{C}(=\mathrm{O}) \mathrm{O}[\mathrm{O}]+\mathrm{NO}_{2} \rightarrow \mathrm{O}=\mathrm{N}(=\mathrm{O}) \mathrm{OOC}(=\mathrm{O}) \mathrm{C}(\mathrm{O}) \mathrm{Cl}$ & TROEMCM & $2.70 \cdot 10^{-28 *(T / 298)^{7.1}}$ & $1.20 \cdot 10^{-11 *(\mathrm{~T} / 298)^{0.9}}$ & 0.3 \\
\hline G358 & $\mathrm{O}=\mathrm{N}(=\mathrm{O}) \mathrm{OOC}(=\mathrm{O}) \mathrm{C}(\mathrm{Cl})(\mathrm{Cl}) \mathrm{Cl} \rightarrow \mathrm{ClC}(\mathrm{Cl})(\mathrm{Cl}) \mathrm{C}(=\mathrm{O}) \mathrm{O}[\mathrm{O}]+\mathrm{NO}_{2}$ & TROEMCM & $4.90 \cdot 10^{-03} \exp (-12100 / \mathrm{T})$ & $5.40 \cdot 10^{+16} \exp (-13830 / \mathrm{T})$ & 0.3 \\
\hline G363 & $\mathrm{ClCC}(=\mathrm{O}) \mathrm{OON}(=\mathrm{O})=\mathrm{O} \rightarrow \mathrm{ClCC}(=\mathrm{O}) \mathrm{O}[\mathrm{O}]+\mathrm{NO}_{2}$ & TROEMCM & $4.90 \cdot 10^{-03} \exp (-12100 / \mathrm{T})$ & $5.40 \cdot 10^{+16} \exp (-13830 / \mathrm{T})$ & 0.3 \\
\hline G367 & $\mathrm{O}=\mathrm{N}(=\mathrm{O}) \mathrm{OOC}(=\mathrm{O}) \mathrm{C}(\mathrm{O})(\mathrm{Cl}) \mathrm{Cl} \rightarrow \mathrm{OC}(\mathrm{Cl})(\mathrm{Cl}) \mathrm{C}(=\mathrm{O}) \mathrm{O}[\mathrm{O}]+\mathrm{NO}_{2}$ & TROEMCM & $4.90 \cdot 10^{-03} \exp (-12100 / \mathrm{T})$ & $5.40 \cdot 10^{+16} \exp (-13830 / \mathrm{T})$ & 0.3 \\
\hline G372 & $\mathrm{ClCC}(\mathrm{Cl}) \mathrm{C}(=\mathrm{O}) \mathrm{OON}(=\mathrm{O})=\mathrm{O} \rightarrow \mathrm{ClCC}(\mathrm{Cl}) \mathrm{C}(=\mathrm{O}) \mathrm{O}[\mathrm{O}]+\mathrm{NO}_{2}$ & TROEMCM & $4.90 \cdot 10^{-03} \exp (-12100 / \mathrm{T})$ & $5.40 \cdot 10^{+16} \exp (-13830 / \mathrm{T})$ & 0.3 \\
\hline G384 & $\mathrm{O}=\mathrm{N}(=\mathrm{O}) \mathrm{OOC}(=\mathrm{O}) \mathrm{C}(\mathrm{Cl}) \mathrm{Cl} \rightarrow \mathrm{ClC}(\mathrm{Cl}) \mathrm{C}(=\mathrm{O}) \mathrm{O}[\mathrm{O}]+\mathrm{NO}_{2}$ & TROEMCM & $4.90 \cdot 10^{-03} \exp (-12100 / \mathrm{T})$ & $5.40 \cdot 10^{+16} \exp (-13830 / \mathrm{T})$ & 0.3 \\
\hline G400 & $\mathrm{O}=\mathrm{N}(=\mathrm{O}) \mathrm{OOC}(=\mathrm{O}) \mathrm{C}(\mathrm{O}) \mathrm{Cl} \rightarrow \mathrm{OC}(\mathrm{Cl}) \mathrm{C}(=\mathrm{O}) \mathrm{O}[\mathrm{O}]+\mathrm{NO}_{2}$ & TROEMCM & $4.90 \cdot 10^{-03} \exp (-12100 / \mathrm{T})$ & $5.40 \cdot 10^{+16} \exp (-13830 / \mathrm{T})$ & 0.3 \\
\hline G468 & $\mathrm{ClO}+\mathrm{ClO} \rightarrow \mathrm{Cl}_{2} \mathrm{O}_{2}$ & TROE & $1.60 \cdot 10^{-32} *(\mathrm{~T} / 298)^{-4.5}$ & $2.00 \cdot 10^{-12} *(\mathrm{~T} / 298)-2.4$ & \\
\hline G469 & $\mathrm{Cl}+\mathrm{O}_{2} \rightarrow \mathrm{ClO}_{2}$ & TROE & $2.20 \cdot 10^{-33 *}(\mathrm{~T} / 298)^{-3.1}$ & $1.80 \cdot 10^{-10}$ & \\
\hline G472 & $\mathrm{Cl}_{2} \mathrm{O}_{2} \rightarrow \mathrm{ClO}+\mathrm{ClO}$ & TROEXP & $3.70 \cdot 10^{-07} \exp (-7690 / \mathrm{T})$ & $7.90 \cdot 10^{+15} \exp (-8820 / \mathrm{T})$ & 0.45 \\
\hline G477 & $\mathrm{OClO}+\mathrm{ClO} \rightarrow \mathrm{Cl}_{2} \mathrm{O}_{3}$ & TROE & $6.20 \cdot 10^{-32 *(T / 298)^{-4.7}}$ & $2.40 \cdot 10^{-11}$ & \\
\hline G478 & $\mathrm{Cl}_{2} \mathrm{O}_{3} \rightarrow \mathrm{OClO}+\mathrm{ClO}$ & TROEXP & $1.40 \cdot 10-10 \exp (-3810 / \mathrm{T})$ & $2.50 \cdot 10^{+12} \exp (-4940 / \mathrm{T})$ & \\
\hline G488 & $\mathrm{Cl}+\mathrm{NO}_{2} \rightarrow \mathrm{ClNO}_{2}$ & TROE & $1.80 \cdot 10^{-31 *}(\mathrm{~T} / 298)^{-2.0}$ & $1.00 \cdot 10^{-10} *(\mathrm{~T} / 298)^{-1.0}$ & \\
\hline G490 & $\mathrm{ClO}+\mathrm{NO}_{2} \rightarrow \mathrm{ClNO}_{3}$ & TROEF & $1.60 \cdot 10^{-31 *(\mathrm{~T} / 298)^{-3.4}}$ & $7.00 \cdot 10^{-11}$ & 0.4 \\
\hline G570 & $\begin{array}{l}\mathrm{Cl}+\mathrm{C} \# \mathrm{C} \rightarrow 0.26 \mathrm{O}=\mathrm{CCl}+0.21 \mathrm{Cl}+0.53 \mathrm{HCl}+0.21 \mathrm{O}=\mathrm{CC}=\mathrm{O}+1.32 \\
\mathrm{CO}+0.79 \mathrm{HO}_{2}\end{array}$ & TROE & $6.10 \cdot 10^{-30 *}(\mathrm{~T} / 298)^{-3.0}$ & $2.00 \cdot 10^{-10}$ & \\
\hline G607 & $\mathrm{Cl}+\mathrm{C}=\mathrm{C} \rightarrow \mathrm{ClCCO}[\mathrm{O}]$ & TROEF & $1.85 \cdot 10^{-29 *}(\mathrm{~T} / 298)^{-3.3}$ & $6.00 \cdot 10^{-10}$ & 0.4 \\
\hline G618 & $\mathrm{CC}(\mathrm{Cl}) \mathrm{C}(=\mathrm{O}) \mathrm{O}[\mathrm{O}]+\mathrm{NO}_{2} \rightarrow \mathrm{CC}(\mathrm{Cl}) \mathrm{C}(=\mathrm{O}) \mathrm{OON}(=\mathrm{O})=\mathrm{O}$ & TROEMCM & $2.70 \cdot 10^{-28 *(T / 298)^{7.1}}$ & $1.20 \cdot 10^{-11 *(\mathrm{~T} / 298)^{0.9}}$ & 0.3 \\
\hline G619 & $\mathrm{CC}(\mathrm{Cl}) \mathrm{C}(=\mathrm{O}) \mathrm{OON}(=\mathrm{O})=\mathrm{O} \rightarrow \mathrm{CC}(\mathrm{Cl}) \mathrm{C}(=\mathrm{O}) \mathrm{O}[\mathrm{O}]+\mathrm{NO}_{2}$ & TROEMCM & $4.90 \cdot 10^{-03} \exp (-12100 / \mathrm{T})$ & $5.40 \cdot 10^{+16} \exp (-13830 / \mathrm{T})$ & 0.3 \\
\hline G629 & {$[\mathrm{O}] \mathrm{OC}(\mathrm{Cl})(\mathrm{Cl}) \mathrm{Cl}+\mathrm{NO}_{2} \rightarrow \mathrm{ClC}(\mathrm{Cl})(\mathrm{Cl}) \mathrm{OON}(=\mathrm{O})=\mathrm{O}$} & TROEF & $9.20 \cdot 10^{-29} *(\mathrm{~T} / 298)^{-6.0}$ & $1.50 \cdot 10^{-12 *}(\mathrm{~T} / 298)^{-0.7}$ & 0.32 \\
\hline G630 & $\mathrm{ClC}(\mathrm{Cl})(\mathrm{Cl}) \mathrm{OON}(=\mathrm{O})=\mathrm{O} \rightarrow[\mathrm{O}] \mathrm{OC}(\mathrm{Cl})(\mathrm{Cl}) \mathrm{Cl}+\mathrm{NO}_{2}$ & TROEXP & $4.30 \cdot 10^{-03} \exp (-10235 / \mathrm{T})$ & $4.80 \cdot 10^{+16} \exp (-13830 / \mathrm{T})$ & 0.32 \\
\hline G646 & $\mathrm{Br}+\mathrm{NO}_{2} \rightarrow \mathrm{BrNO}_{2}$ & TROEF & $4.20 \cdot 10^{-31 *(T / 298)^{-2.4}}$ & $2.70 \cdot 10^{-11}$ & 0.55 \\
\hline G649 & $\mathrm{BrO}+\mathrm{NO}_{2} \rightarrow \mathrm{BrNO}_{3}$ & TROEF & $4.70 \cdot 10^{-31 *(T / 298)^{-3.1}}$ & $1.80 \cdot 10^{-11}$ & 0.4 \\
\hline G714 & $\mathrm{BrC}(\mathrm{Br})(\mathrm{Br}) \mathrm{O}[\mathrm{O}]+\mathrm{NO}_{2} \rightarrow \mathrm{BrC}(\mathrm{Br})(\mathrm{Br}) \mathrm{OON}(=\mathrm{O})=\mathrm{O}$ & TROEF & $9.20 \cdot 10^{-29} *(\mathrm{~T} / 298)^{-6.0}$ & $1.50 \cdot 10^{-12 *}(\mathrm{~T} / 298)^{-0.7}$ & 0.32 \\
\hline G715 & $\mathrm{BrC}(\mathrm{Br})(\mathrm{Br}) \mathrm{OON}(=\mathrm{O})=\mathrm{O} \rightarrow \mathrm{BrC}(\mathrm{Br})(\mathrm{Br}) \mathrm{O}[\mathrm{O}]+\mathrm{NO}_{2}$ & TROEXP & $4.30 \cdot 10^{-03} \exp (-10235 / \mathrm{T})$ & $4.80 \cdot 10^{+16} \exp (-11820 / \mathrm{T})$ & 0.32 \\
\hline
\end{tabular}




\begin{tabular}{|c|c|c|c|c|c|}
\hline & Reaction & TYPE & $\mathbf{k}_{\mathbf{0}}$ & $k \infty$ & $\mathbf{F}$ \\
\hline G756 & $\operatorname{BrCC}(=\mathrm{O}) \mathrm{O}[\mathrm{O}]+\mathrm{NO}_{2} \rightarrow \operatorname{BrCC}(=\mathrm{O}) \mathrm{OON}(=\mathrm{O})=\mathrm{O}$ & TROEMCM & $2.70 \cdot 10^{-28 *(T / 298)^{7.1}}$ & $1.20 \cdot 10^{-11 *(\mathrm{~T} / 298)^{0.9}}$ & 0.3 \\
\hline G763 & $\operatorname{BrCC}(=\mathrm{O}) \mathrm{OON}(=\mathrm{O})=\mathrm{O} \rightarrow \operatorname{BrCC}(=\mathrm{O}) \mathrm{O}[\mathrm{O}]+\mathrm{NO}_{2}$ & TROEMCM & $4.90 \cdot 10^{-03} \exp (-12100 / \mathrm{T})$ & $5.40 \cdot 10^{+16} \exp (-13830 / \mathrm{T})$ & 0.3 \\
\hline G779 & $\mathrm{I}+\mathrm{NO} \rightarrow \mathrm{INO}$ & TROE & $1.80 \cdot 10^{-32 *(\mathrm{~T} / 298)^{-1.0}}$ & $1.70 \cdot 10^{-11}$ & \\
\hline G780 & $\mathrm{I}+\mathrm{NO}_{2} \rightarrow \mathrm{INO}_{2}$ & TROEF & $3.00 \cdot 10^{-31 *(\mathrm{~T} / 298)^{-1.0}}$ & $6.60 \cdot 10^{-11}$ & \\
\hline G784 & $\mathrm{IO}+\mathrm{NO}_{2} \rightarrow \mathrm{INO}_{3}$ & TROEF & $7.70 \cdot 10^{-31}(\mathrm{~T} / 300)^{-5.0}$ & $1.60 \cdot 10^{-11}$ & \\
\hline G813 & $\mathrm{ICC}(=\mathrm{O}) \mathrm{O}[\mathrm{O}]+\mathrm{NO}_{2} \rightarrow \mathrm{ICC}(=\mathrm{O}) \mathrm{OON}(=\mathrm{O})=\mathrm{O}$ & TROEF & $2.70 \cdot 10^{-28 *}(\mathrm{~T} / 298)^{7.1}$ & $1.20 \cdot 10^{-11 *(\mathrm{~T} / 298)^{0.9}}$ & 0.3 \\
\hline G814 & $\mathrm{ICC}(=\mathrm{O}) \mathrm{OON}(=\mathrm{O})=\mathrm{O} \rightarrow \mathrm{ICC}(=\mathrm{O}) \mathrm{O}[\mathrm{O}]+\mathrm{NO}_{2}$ & TROEXP & $4.90 \cdot 10^{-03} \exp (-12100 / \mathrm{T})$ & $5.40 \cdot 10^{+16} \exp (-13830 / \mathrm{T})$ & 0.3 \\
\hline
\end{tabular}


Table S6 Uptake processes implemented within the CAPRAM-HM3.0.

\begin{tabular}{|c|c|c|c|c|c|c|c|c|}
\hline & Species & $\mathrm{K}_{\mathrm{H} 298,\left[\mathrm{M} \mathrm{atm}^{-1}\right]}$ & $-\Delta \mathbf{H} / \mathbf{R},[\mathbf{K}]$ & Ref. & $\alpha$ & Ref. & $D_{g 298}\left[10^{5} \mathrm{~m}^{2} \mathrm{~s}^{-1}\right]$ & Ref. \\
\hline H1 (2) & $\mathrm{Cl}_{2}$ & $9.15 \cdot 10^{-2}$ & 2490 & 12 & 0.08 & 12 & 1.28 & 12 \\
\hline $\mathrm{H} 2$ (2) & $\mathrm{Cl}$ & $2.00 \cdot 10^{-1}$ & & 12 & 0.05 & 12 & 1.82 & 12 \\
\hline $\mathrm{H} 3 \mathrm{Q}$ & $\mathrm{ClO}$ & $5.50 \cdot 10^{0}$ & & 106 & 0.064 & 12 & 1.55 & 12 \\
\hline H4(2) & $\mathrm{ClO}_{2}$ & $1.00 \cdot 10^{0}$ & -3300 & 12 & 0.05 & 12 & 1.39 & 12 \\
\hline H5 (2) & $\mathrm{HCl}$ & $1.10 \cdot 10^{0}$ & 2020 & 12 & 0.1026 & 12 & 1.89 & 12 \\
\hline H6(2) & $\mathrm{HOCl}$ & $6.60 \cdot 10^{2}$ & 5862 & 12 & 0.5 & 12 & 1.51 & 12 \\
\hline H7(2) & CINO & $5.00 \cdot 10^{-2}$ & & 12 & 0.01 & 12 & 1.39 & 12 \\
\hline H8(2) & $\mathrm{ClNO}_{2}$ & $2.40 \cdot 10^{-2}$ & & 12 & 0.01 & 12 & 1.27 & 12 \\
\hline H9(2) & $\mathrm{ClNO}_{3}$ & $2.10 \cdot 10^{5}$ & 8700 & 12 & 0.1 & 12 & 1.18 & 12 \\
\hline $\mathrm{H} 10$ (2) & $\mathrm{ClCH}_{2} \mathrm{CO}\left(\mathrm{O}_{2}\right)$ & $6.69 \cdot 10^{2}$ & 5893 & 12 & 0.019 & 12 & 0.94 & 12 \\
\hline $\mathrm{H} 110$ & $\mathrm{ClCH}_{2} \mathrm{COOH}$ & $1.11 \cdot 10^{5}$ & 9700 & 107 & 0.1 & 12 & 0.97 & 12 \\
\hline $\mathrm{H} 12$ (2) & $\mathrm{CH}_{3} \mathrm{COCOCl}$ & $1.40 \cdot 10^{0}$ & 7541 & 12 & 0.03 & 12 & 0.88 & 12 \\
\hline $\mathrm{H} 130$ & $\mathrm{COCl}_{2}$ & $5.98 \cdot 10^{-2}$ & 3800 & 108 & 0.02 & 12 & 1.02 & 12 \\
\hline $\mathrm{H} 14$ (2) & $\mathrm{CHOCl}$ & $3.00 \cdot 10^{3}$ & 7216 & 12 & 0.02 & 12 & 1.23 & 12 \\
\hline H15 & $\mathrm{ClCH}_{2} \mathrm{CHO}$ & $4.15 \cdot 10^{1}$ & & 107 & 0.03 & as $\mathrm{CH}_{3} \mathrm{CHO}$ & 1.19 & 109 \\
\hline H16 & $\mathrm{Cl}_{2} \mathrm{CHCHO}$ & $1.22 \cdot 10^{2}$ & & 107 & 0.03 & as $\mathrm{CH}_{3} \mathrm{CHO}$ & 1.16 & 109 \\
\hline H17 & $\mathrm{CH}_{3} \mathrm{COCl}$ & $4.15 \cdot 10^{1}$ & & estimated as $\mathrm{ClCH}_{2} \mathrm{CHO}$ & 0.03 & as $\mathrm{CH}_{3} \mathrm{CHO}$ & 1.19 & 109 \\
\hline H18 & $\mathrm{ClCH}_{2} \mathrm{COCl}$ & $4.36 \cdot 10^{0}$ & & 107 & 0.03 & as $\mathrm{CH}_{3} \mathrm{CHO}$ & 1.16 & 109 \\
\hline H19 & $\mathrm{ClCH}_{2} \mathrm{CH}_{2} \mathrm{OH}$ & $9.63 \cdot 10^{3}$ & & 107 & 0.0176 & as $\mathrm{CH}_{3} \mathrm{CH}_{2} \mathrm{OH}$ & 1.14 & 109 \\
\hline $\mathrm{H} 20$ & $\mathrm{CH}_{3} \mathrm{CHOHCH}_{2} \mathrm{Cl}$ & $5.88 \cdot 10^{2}$ & & 107 & 0.013 & as $\mathrm{CH}_{3} \mathrm{CHOHCH}_{3}$ & 0.98 & 109 \\
\hline $\mathrm{H} 21$ & $\mathrm{Cl}_{3} \mathrm{CCHO}$ & $3.45 \cdot 10^{5}$ & 3500 & 107 & 0.03 & as $\mathrm{CH}_{3} \mathrm{CHO}$ & 1.15 & 109 \\
\hline $\mathrm{H} 22$ & $\mathrm{CH}_{3} \mathrm{COCH}_{2} \mathrm{Cl}$ & $5.88 \cdot 10^{1}$ & 5400 & 107 & 0.0054 & as $\mathrm{CH}_{3} \mathrm{COCH}_{3}$ & 1.00 & 109 \\
\hline $\mathrm{H} 23$ & $\mathrm{Cl}_{2} \mathrm{CHCOOH}$ & $1.22 \cdot 10^{5}$ & 8000 & 107 & 0.1 & as $\mathrm{ClCH}_{2} \mathrm{COOH}$ & 1.09 & 109 \\
\hline $\mathrm{H} 24$ & $\mathrm{o}-\mathrm{C}_{6} \mathrm{H}_{5} \mathrm{ClO}$ & $1.52 \cdot 10^{2}$ & & 107 & 0.1 & estimated & 0.77 & 109 \\
\hline $\mathrm{H} 25$ & $\mathrm{p}-\mathrm{C}_{6} \mathrm{H}_{5} \mathrm{ClO}$ & $1.42 \cdot 10^{5}$ & 11000 & 107 & 0.1 & estimated & 0.77 & 109 \\
\hline $\mathrm{H} 26$ (2) & $\mathrm{Br}_{2}$ & $7.60 \cdot 10^{-1}$ & 4100 & 12 & 0.08 & 12 & 1.00 & 12 \\
\hline $\mathrm{H} 27$ (2) & $\mathrm{Br}$ & $1.20 \cdot 10^{0}$ & & 12 & 0.05 & 12 & 1.29 & 12 \\
\hline $\mathrm{H} 280$ & $\mathrm{BrO}$ & $5.50 \cdot 10^{0}$ & & estimated as $\mathrm{ClO}$ & 0.06 & 12 & 1.19 & 12 \\
\hline $\mathrm{H} 29$ (2) & $\mathrm{HBr}$ & $1.30 \cdot 10^{0}$ & 10239 & 12 & 0.0481 & 12 & 1.26 & 12 \\
\hline
\end{tabular}




\begin{tabular}{|c|c|c|c|c|c|c|c|c|}
\hline & Species & $\mathrm{K}_{\mathrm{H} 298},\left[\mathrm{M} \mathrm{atm}^{-1}\right]$ & $-\Delta \mathbf{H} / \mathbf{R},[\mathbf{K}]$ & Ref. & $\alpha$ & Ref. & $D_{g 298}\left[10^{5} \mathrm{~m}^{2} \mathrm{~s}^{-1}\right]$ & Ref. \\
\hline $\mathrm{H} 30$ (2) & $\mathrm{HOBr}$ & $9.30 \cdot 10^{1}$ & 5862 & 12 & 0.5 & 12 & 1.16 & 12 \\
\hline H31 (2) & $\mathrm{BrNO}_{2}$ & $3.00 \cdot 10^{-1}$ & & 12 & 0.01 & 12 & 1.06 & 12 \\
\hline H32 (2) & $\mathrm{BrNO}_{3}$ & $2.10 \cdot 10^{5}$ & 8700 & 12 & 0.8 & 12 & 1.01 & 12 \\
\hline H33 (2) & $\mathrm{BrCl}$ & $9.40 \cdot 10^{-1}$ & -5600 & 12 & 0.33 & 12 & 1.05 & 12 \\
\hline H34(2) & $\mathrm{BrCH}_{2} \mathrm{CO}\left(\mathrm{O}_{2}\right)$ & $6.69 \cdot 10^{2}$ & 5893 & 12 & 0.019 & 12 & 0.84 & 12 \\
\hline $\mathrm{H} 35 \mathrm{O}$ & $\mathrm{BrCH}_{2} \mathrm{COOH}$ & $1.52 \cdot 10^{5}$ & 9300 & 107 & 0.0322 & 12 & 0.84 & 12 \\
\hline $\mathrm{H} 36(2)$ & $\mathrm{CH}_{3} \mathrm{COCOBr}$ & $1.40 \cdot 10^{0}$ & 7541 & 12 & 0.03 & 12 & 0.79 & 12 \\
\hline $\mathrm{H} 370$ & $\mathrm{COBr}_{2}$ & $2.13 \cdot 10^{1}$ & & 107 & 0.02 & 12 & 0.81 & 12 \\
\hline $\mathrm{H} 380$ & $\mathrm{CHOBr}$ & $7.40 \cdot 10^{1}$ & & 107 & 0.02 & 12 & 1.02 & 12 \\
\hline H39 & $\mathrm{BrCH}_{2} \mathrm{OH}$ & $2.03 \cdot 10^{3}$ & & 107 & 0.0271 & as $\mathrm{CH}_{3} \mathrm{OH}$ & 1.35 & 109 \\
\hline $\mathrm{H} 40$ & $\mathrm{Br}_{2} \mathrm{CHOH}$ & $1.72 \cdot 10^{4}$ & & 107 & 0.0271 & as $\mathrm{CH}_{3} \mathrm{OH}$ & 1.32 & 109 \\
\hline H41 & $\mathrm{Br}_{3} \mathrm{COH}$ & $1.52 \cdot 10^{5}$ & & 107 & 0.0271 & as $\mathrm{CH}_{3} \mathrm{OH}$ & 1.33 & 109 \\
\hline H42 & $\mathrm{BrCH}_{2} \mathrm{OOH}$ & $2.53 \cdot 10^{3}$ & & 107 & 0.006758 & as $\mathrm{CH}_{3} \mathrm{OOH}$ & 1.25 & 109 \\
\hline $\mathrm{H} 43$ & $\mathrm{CH}_{3} \mathrm{COCH}_{2} \mathrm{Br}$ & $1.72 \cdot 10^{2}$ & & 107 & 0.0054 & as $\mathrm{CH}_{3} \mathrm{COCH}_{3}$ & 0.96 & 109 \\
\hline H44(2) & $\mathrm{I}_{2}$ & $3.00 \cdot 10^{0}$ & 4431 & 12 & 0.0126 & 12 & 0.86 & 12 \\
\hline $\mathrm{H} 45$ (2) & $\mathrm{I}$ & $8.00 \cdot 10^{-2}$ & & 12 & 0.05 & 12 & 1.16 & 12 \\
\hline $\mathrm{H} 460$ & $\mathrm{IO}$ & $5.50 \cdot 10^{0}$ & & estimated as $\mathrm{ClO}$ & 0.558 & 12 & 1.10 & 12 \\
\hline $\mathrm{H} 47 \mathrm{O}$ & $\mathrm{OIO}$ & $1.00 \cdot 10^{4}$ & & 107 & 1 & 12 & 1.04 & 12 \\
\hline $\mathrm{H} 48 \mathrm{O}$ & $\mathrm{I}_{2} \mathrm{O}_{2}$ & $1.00 \cdot 10^{4}$ & & 107 & 0.123 & 12 & 0.80 & 12 \\
\hline H49 & $\mathrm{I}_{2} \mathrm{O}_{3}$ & $1.00 \cdot 10^{4}$ & & 107 & 0.1 & estimated & 0.80 & 109 \\
\hline H50 & $\mathrm{I}_{2} \mathrm{O}_{4}$ & $1.00 \cdot 10^{4}$ & & 107 & 0.1 & estimated & 0.80 & 109 \\
\hline H51 (2) & HI & $2.50 \cdot 10^{0}$ & 9800 & 12 & 0.057 & 12 & 1.14 & 12 \\
\hline H52(2) & HOI & $4.50 \cdot 10^{2}$ & 5862 & 12 & 0.5 & 12 & 1.08 & 12 \\
\hline $\mathrm{H} 53$ (2) & $\mathrm{HIO}_{3}$ & $2.10 \cdot 10^{5}$ & 8700 & 12 & 0.0126 & 12 & 0.98 & 12 \\
\hline H54(2) & $\mathrm{INO}_{2}$ & $2.10 \cdot 10^{5}$ & 8700 & 12 & 0.123 & 12 & 0.99 & 12 \\
\hline H55 (2) & $\mathrm{INO}_{3}$ & $2.10 \cdot 10^{5}$ & 8700 & 12 & 0.123 & 12 & 0.96 & 12 \\
\hline H56(2) & $\mathrm{ICl}$ & $1.10 \cdot 10^{2}$ & 5600 & 12 & 0.0126 & 12 & 0.98 & 12 \\
\hline H57(2) & $\mathrm{IBr}$ & $2.40 \cdot 10^{1}$ & 5600 & 12 & 0.0126 & 12 & 0.88 & 12 \\
\hline H58(2) & $\mathrm{ICH}_{2} \mathrm{CO}\left(\mathrm{O}_{2}\right)$ & $6.69 \cdot 10^{2}$ & 5893 & 12 & 0.019 & 12 & 0.80 & 12 \\
\hline H59 & $\mathrm{ICH}_{2} \mathrm{COOH}$ & $2.43 \cdot 10^{4}$ & & 107 & 0.322 & 12 & 0.82 & 12 \\
\hline $\mathrm{H} 60$ (2) & $\mathrm{COI}_{2}$ & $7.00 \cdot 10^{-2}$ & & 12 & 0.02 & 12 & 0.76 & 12 \\
\hline H61 (2) & $\mathrm{CHOI}$ & $3.00 \cdot 10^{3}$ & 7216 & 12 & 0.02 & 12 & 0.97 & 12 \\
\hline
\end{tabular}


Table S7 Irreversible aqueous-phase reactions implemented within the CAPRAM-HM3.0.

(2) already implemented in CAPRAM-HM2.1, $Q$ update of CAPRAM-HM2.1, new reactions in blue

\begin{tabular}{|c|c|c|c|c|}
\hline & Reaction & $\mathbf{k}_{\mathbf{2 9 8}}$ & $\mathbf{E}_{\mathrm{A}} / \mathbf{R}$ & Reference \\
\hline A1 (2) & $\mathrm{Cl}+\mathrm{Cl} \rightarrow \mathrm{Cl}_{2}$ & $8.75 \cdot 10^{7}$ & & \\
\hline A2(2) & $\mathrm{Cl}_{2}^{-}+\mathrm{Cl} \rightarrow \mathrm{Cl}_{2}+\mathrm{Cl}-$ & $2.10 \cdot 10^{9}$ & & \\
\hline A3 (2) & $\mathrm{Cl}_{2}^{-}+\mathrm{Cl}_{2}^{-} \rightarrow \mathrm{Cl}_{2}+2 \mathrm{Cl}-$ & $1.80 \cdot 10^{9}$ & & \\
\hline $\mathrm{A} 4(2)$ & $\mathrm{Cl}^{-}+\mathrm{O}_{3} \rightarrow \mathrm{ClO}^{-}+\mathrm{O}_{2}$ & $3.00 \cdot 10^{-3}$ & & \\
\hline $\mathrm{A} 5$ (2) & $\mathrm{Cl}+\mathrm{H}_{2} \mathrm{O}_{2} \rightarrow \mathrm{H}^{+}+\mathrm{Cl}^{-}+\mathrm{HO}_{2}$ & $2.00 \cdot 10^{9}$ & & \\
\hline $\mathrm{A} 6 \mathrm{Q}$ & $\mathrm{Cl}_{2}^{-}+\mathrm{H}_{2} \mathrm{O}_{2} \rightarrow 2 \mathrm{Cl}^{-}+\mathrm{H}^{+}+\mathrm{HO}_{2}$ & $6.20 \cdot 10^{5}$ & 3340 & $\mathrm{k}_{298}$ after $\mathrm{Yu}{ }^{110}, \mathrm{E}_{\mathrm{A}} / \mathrm{R}$ after Jacobi et al. ${ }^{111}$ \\
\hline A7(2) & $\mathrm{Cl}_{2}^{-}+\mathrm{H}_{2} \mathrm{O} \rightarrow \mathrm{H}^{+}+\mathrm{Cl}^{-}+\mathrm{ClOH}^{-}$ & $2.34 \cdot 10^{1}$ & & \\
\hline $\mathrm{A} 8(2)$ & $\mathrm{Cl}_{2}^{-}+\mathrm{HO}_{2} \rightarrow 2 \mathrm{Cl}^{-}+\mathrm{H}^{+}+\mathrm{O}_{2}$ & $1.30 \cdot 10^{10}$ & & \\
\hline A9(2) & $\mathrm{Cl}_{2}^{-}+\mathrm{O}_{2}^{-} \rightarrow 2 \mathrm{Cl}^{-}+\mathrm{O}_{2}$ & $6.00 \cdot 10^{9}$ & & \\
\hline A10(2) & $\mathrm{Cl}_{2}^{-}+\mathrm{OH} \rightarrow \mathrm{HOCl}+\mathrm{Cl}-$ & $1.00 \cdot 10^{9}$ & & \\
\hline A11 (2) & $\mathrm{Cl}_{2}^{-}+\mathrm{OH}^{-} \rightarrow 2 \mathrm{Cl}^{-}+\mathrm{OH}$ & $4.00 \cdot 10^{6}$ & & \\
\hline A12(2) & $\mathrm{Cl}_{3}^{-}+\mathrm{HO}_{2} \rightarrow \mathrm{Cl}_{2}^{-}+\mathrm{H}^{+}+\mathrm{Cl}-+\mathrm{O}_{2}$ & $1.00 \cdot 10^{9}$ & & \\
\hline A132) & $\mathrm{Cl}_{3}^{-}+\mathrm{O}_{2}^{-} \rightarrow \mathrm{Cl}_{2}^{-}+\mathrm{Cl}^{-}+\mathrm{O}_{2}$ & $3.80 \cdot 10^{9}$ & & \\
\hline A14(2) & $\mathrm{Cl}_{2}+\mathrm{HO}_{2} \rightarrow \mathrm{Cl}_{2}^{-}+\mathrm{H}^{+}+\mathrm{O}_{2}$ & $1.00 \cdot 10^{9}$ & & \\
\hline A15 (2) & $\mathrm{Cl}_{2}+\mathrm{O}_{2}^{-} \rightarrow \mathrm{Cl}_{2}^{-}+\mathrm{O}_{2}$ & $1.00 \cdot 10^{9}$ & & \\
\hline A16 8 & $\mathrm{HOCl}+\mathrm{H}_{2} \mathrm{O}_{2} \rightarrow \mathrm{H}^{+}+\mathrm{Cl}^{-}+\mathrm{H}_{2} \mathrm{O}+\mathrm{O}_{2}$ & $8.17 \cdot 10^{-1}$ & & revised, Connick ${ }^{112}$ \\
\hline A170 & $\mathrm{ClO}^{-}+\mathrm{H}_{2} \mathrm{O}_{2} \rightarrow \mathrm{Cl}^{-}+\mathrm{H}_{2} \mathrm{O}+\mathrm{O}_{2}$ & $2.83 \cdot 10^{3}$ & 5840 & revised, Connick ${ }^{112}$ \\
\hline A18(2) & $\mathrm{HOCl}+\mathrm{HO}_{2} \rightarrow \mathrm{Cl}+\mathrm{H}_{2} \mathrm{O}+\mathrm{O}_{2}$ & $7.50 \cdot 10^{6}$ & & \\
\hline A190 & $\mathrm{HOCl}+\mathrm{O}_{2}^{-} \rightarrow \mathrm{ClOH}^{-}+\mathrm{O}_{2}$ & $7.50 \cdot 10^{6}$ & & product after reaction $\mathrm{HOBr}$ with $\mathrm{O}_{2}^{-}$ \\
\hline A20 (2) & $\mathrm{ClO}^{-}+\mathrm{O}_{2}^{-} \rightarrow \mathrm{Cl}+2 \mathrm{OH}^{-}+\mathrm{O}_{2}-\mathrm{H}_{2} \mathrm{O}$ & $2.00 \cdot 10^{8}$ & & \\
\hline A21 (2) & $\mathrm{HOCl}+\mathrm{OH} \rightarrow \mathrm{ClO}+\mathrm{H}_{2} \mathrm{O}$ & $2.00 \cdot 10^{9}$ & & \\
\hline $\mathrm{A} 22(2)$ & $\mathrm{ClO}^{-}+\mathrm{OH} \rightarrow \mathrm{ClO}+\mathrm{OH}^{-}$ & $8.80 \cdot 10^{9}$ & & \\
\hline $\mathrm{A} 23$ (2) & $\mathrm{Cl}_{2}^{-}+\mathrm{HSO}_{3}^{-} \rightarrow 2 \mathrm{Cl}^{-}+\mathrm{H}^{+}+\mathrm{SO}_{3}^{-}$ & $1.70 \cdot 10^{8}$ & 400 & \\
\hline A24(2) & $\mathrm{Cl}_{2}^{-}+\mathrm{SO}_{3}^{2-} \rightarrow 2 \mathrm{Cl}^{-}+\mathrm{SO}_{3}^{-}$ & $6.20 \cdot 10^{7}$ & & \\
\hline $\mathrm{A} 25$ (2) & $\mathrm{HOCl}+\mathrm{SO}_{3}^{2-} \rightarrow \mathrm{Cl}-+\mathrm{HSO}_{4}^{-}$ & $7.60 \cdot 10^{8}$ & & \\
\hline A26(2) & $\mathrm{HOCl}+\mathrm{HSO}_{3}^{-} \rightarrow \mathrm{Cl}^{-}+\mathrm{H}^{+}+\mathrm{HSO}_{4}{ }^{2-}$ & $7.60 \cdot 10^{8}$ & & \\
\hline A27(2) & $\mathrm{Cl}^{-}+\mathrm{HSO}_{5}^{-} \rightarrow \mathrm{HOCl}+\mathrm{SO}_{4}^{2-}$ & $1.80 \cdot 10^{-3}$ & 7352 & \\
\hline A28(2) & $\mathrm{Cl}_{2}^{-}+\mathrm{CH}_{2} \mathrm{OHSO}_{3}^{-} \rightarrow 2 \mathrm{Cl}^{-}+\mathrm{CH}_{2} \mathrm{OHSO}_{3}$ & $5.00 \cdot 10^{5}$ & & \\
\hline
\end{tabular}


(2) already implemented in CAPRAM-HM2.1, $\odot$ update of CAPRAM-HM2.1, new reactions in blue

\begin{tabular}{|c|c|c|c|c|}
\hline & Reaction & $\mathbf{k}_{\mathbf{2 9 8}}$ & $\mathbf{E}_{\mathrm{A}} / \mathbf{R}$ & Reference \\
\hline A29(2) & $\mathrm{Cl}_{2}^{-}+\mathrm{NO}_{2}^{-} \rightarrow 2 \mathrm{Cl}^{-}+\mathrm{NO}_{2}$ & $6.00 \cdot 10^{7}$ & & \\
\hline A30(2) & $\mathrm{Cl}_{2}^{-}+\mathrm{Fe}_{2}^{+} \rightarrow 2 \mathrm{Cl}^{-}+\mathrm{Fe}_{3}^{+}$ & $1.00 \cdot 10^{7}$ & 3030 & \\
\hline A31 (2) & $\mathrm{Cl}_{2}^{-}+\mathrm{Fe}_{2}^{+} \rightarrow \mathrm{FeCl}^{2+}+\mathrm{Cl}^{-}$ & $4.00 \cdot 10^{6}$ & 3490 & \\
\hline A32(2) & $\mathrm{Cl}^{-}+\mathrm{FeO}_{2}^{+} \rightarrow \mathrm{Fe}_{3}^{+}+\mathrm{ClOH}^{-}+\mathrm{OH}^{-}-\mathrm{H}_{2} \mathrm{O}$ & $1.00 \cdot 10^{2}$ & & \\
\hline A33(2) & $\mathrm{Cl}_{2}^{-}+\mathrm{Mn}_{2}^{+} \rightarrow \mathrm{MnCl}_{2}^{+}$ & $2.00 \cdot 10^{7}$ & 4090 & \\
\hline A34(2) & $\mathrm{MnCl}_{2}^{+} \rightarrow \mathrm{Cl}_{2}^{-}+\mathrm{Mn}_{2}^{+}$ & $3.00 \cdot 10^{5}$ & & \\
\hline A35 (2) & $\mathrm{MnCl}_{2}^{+} \rightarrow 2 \mathrm{Cl}^{-}+\mathrm{Mn}_{3}^{+}$ & $2.10 \cdot 10^{5}$ & & \\
\hline A36(2) & $\mathrm{Cl}_{2}^{-}+\mathrm{Cu}^{+} \rightarrow 2 \mathrm{Cl}^{-}+\mathrm{Cu}_{2}^{+}$ & $1.00 \cdot 10^{8}$ & & \\
\hline A37(2) & $\mathrm{Cl}+\mathrm{CO}_{3}^{2-} \rightarrow \mathrm{Cl}^{-}+\mathrm{CO}_{3}^{-}$ & $5.00 \cdot 10^{8}$ & & \\
\hline A38(2) & $\mathrm{Cl}+\mathrm{HCO}_{3}^{-} \rightarrow \mathrm{Cl}^{-}+\mathrm{H}^{+}+\mathrm{CO}_{3}^{-}$ & $2.20 \cdot 10^{8}$ & & \\
\hline A39(2) & $\mathrm{Cl}_{2}^{-}+\mathrm{CO}_{3}^{2-} \rightarrow 2 \mathrm{Cl}^{-}+\mathrm{CO}_{3}^{-}$ & $2.70 \cdot 10^{6}$ & & \\
\hline A40 (2) & $\mathrm{Cl}_{2}^{-}+\mathrm{HCO}_{3}^{-} \rightarrow 2 \mathrm{Cl}^{-}+\mathrm{H}^{+}+\mathrm{CO}_{3}^{-}$ & $2.70 \cdot 10^{6}$ & & \\
\hline A41 (2) & $\mathrm{ClNO}_{3} \rightarrow \mathrm{HOCl}+\mathrm{HNO}_{3}$ & $1.62 \cdot 10^{6}$ & 2800 & \\
\hline A42(2) & $\mathrm{ClNO}_{3}+\mathrm{H}^{+} \rightarrow \mathrm{HOCl}+\mathrm{NO}_{2}^{+}$ & $1.12 \cdot 10^{3}$ & 6200 & \\
\hline A43 & $\mathrm{Cl}^{-}+\mathrm{H}_{2} \mathrm{O}_{2} \rightarrow \mathrm{ClO}^{-}+\mathrm{H}_{2} \mathrm{O}$ & $1.10 \cdot 10^{-7}$ & 11881 & Mohammad and Liebhafsky ${ }^{113}$ \\
\hline A44 & $\mathrm{Cl}^{-}+\mathrm{H}_{2} \mathrm{O}_{2}+\mathrm{H}^{+} \rightarrow \mathrm{HOCl}+\mathrm{H}_{2} \mathrm{O}$ & $2.30 \cdot 10^{-5}$ & 10622 & Mohammad and Liebhafsky ${ }^{113}$ \\
\hline A 45 & $\mathrm{Fe}^{2+}+\mathrm{HOCl} \rightarrow \mathrm{Fe}^{3+}+\mathrm{Cl}^{-}+\mathrm{OH}$ & $1.70 \cdot 10^{4}$ & & Folkes et al. ${ }^{114}$ \\
\hline A46 & $\mathrm{Fe}^{2+}+\mathrm{ClO}_{2} \rightarrow \mathrm{Fe}^{3+}+\mathrm{ClO}_{2}^{-}$ & $3.00 \cdot 10^{3}$ & & Hoigne and Bader ${ }^{115}$ \\
\hline A47 & $\mathrm{Fe}^{2+}+\mathrm{ClO}_{3} \rightarrow \mathrm{Fe}^{3+}+\mathrm{ClO}_{3}^{-}$ & $7.00 \cdot 10^{7}$ & & Domae et al. ${ }^{116}$ \\
\hline A 48 & $\mathrm{ClO}^{-}+\mathrm{O}_{3} \rightarrow 0.79 \mathrm{Cl}^{-}+0.21 \mathrm{ClO}_{2}^{-}+1.79 \mathrm{O}_{2}$ & $1.40 \cdot 10^{2}$ & & Haag and Hoigne ${ }^{117}$ \\
\hline A49 & $\mathrm{ClO}+\mathrm{O}_{3}^{-} \rightarrow \mathrm{ClO}^{-}+\mathrm{O}_{3}$ & $1.00 \cdot 10^{9}$ & & Klaning et al. ${ }^{118}$ \\
\hline A50 & $\mathrm{ClO}_{2}+\mathrm{NO}_{2}^{-} \rightarrow \mathrm{ClO}_{2}^{-}+\mathrm{NO}_{2}$ & $1.13 \cdot 10^{2}$ & & Hoigne and Bader ${ }^{115}$ \\
\hline A51 & $\mathrm{ClO}_{2}+\mathrm{O}_{3} \rightarrow \mathrm{ClO}_{3}+\mathrm{O}_{2}$ & $1.37 \cdot 10^{3}$ & & Hoigne and Bader ${ }^{115}$ \\
\hline A52 & $\mathrm{ClO}_{2}+\mathrm{OH} \rightarrow \mathrm{ClO}_{3}^{-}+\mathrm{H}^{+}$ & $4.00 \cdot 10^{9}$ & & Klaning et al. ${ }^{118}$ \\
\hline A53 & $\mathrm{Cl}_{2}^{-}+\mathrm{O}_{3} \rightarrow \mathrm{ClO}^{-}+\mathrm{Cl}+\mathrm{O}_{2}$ & $9.00 \cdot 10^{7}$ & & Bielski ${ }^{119}$, products after Knipping and Dabdub ${ }^{120}$ \\
\hline A54 & $\mathrm{ClO}_{2}+\mathrm{SO}_{3}^{2-} \rightarrow \mathrm{ClO}_{2}^{-}+\mathrm{SO}_{3}^{-}$ & $2.70 \cdot 10^{6}$ & & Huie and Neta ${ }^{121}$ \\
\hline A55 & $\mathrm{ClO}_{2}+\mathrm{O}_{2}^{-} \rightarrow \mathrm{ClO}_{2}^{-}+\mathrm{O}_{2}$ & $3.00 \cdot 10^{9}$ & & Huie and Neta ${ }^{121}$ \\
\hline A56 & $\mathrm{ClO}_{2}^{-}+\mathrm{OH} \rightarrow \mathrm{ClO}_{2}+\mathrm{OH}^{-}$ & $6.10 \cdot 10^{9}$ & & Buxton and Subhani ${ }^{122}$ \\
\hline A57 & $\mathrm{ClO}_{2} \rightarrow \mathrm{Cl}+\mathrm{O}_{2}$ & $6.70 \cdot 10^{9}$ & & Dunn and Simon ${ }^{123}$ \\
\hline A58 & $\mathrm{HOCl}+\mathrm{CH}_{3} \mathrm{COCOO}^{-} \rightarrow \mathrm{CH}_{3} \mathrm{COOH}+\mathrm{Cl}^{-}+\mathrm{CO}_{2}$ & $2.17 \cdot 10^{0}$ & & Galajda et al. ${ }^{124}$ \\
\hline A59 & $\mathrm{HClO}_{2}+\mathrm{HCHO} \rightarrow \mathrm{HCOOH}+\mathrm{HOCl}$ & $1.20 \cdot 10^{4}$ & 6495 & Lehtimaa et al. ${ }^{125}$ \\
\hline
\end{tabular}


(2) already implemented in CAPRAM-HM2.1, $\odot$ update of CAPRAM-HM2.1, new reactions in blue

\begin{tabular}{|c|c|c|c|c|}
\hline & Reaction & $\mathbf{k}_{\mathbf{2 9 8}}$ & $\mathbf{E}_{\mathrm{A}} / \mathbf{R}$ & Reference \\
\hline A60 & $\mathrm{HClO}_{2}+\mathrm{HOCH}_{2} \mathrm{CHO} \rightarrow \mathrm{HOCH}_{2} \mathrm{COOH}+\mathrm{HOCl}$ & $4.50 \cdot 10^{2}$ & 5413 & Lehtimaa et al. ${ }^{125}$ \\
\hline A61 & $\mathrm{Cl}_{2}+\mathrm{NO}_{2}^{-} \rightarrow \mathrm{ClNO}_{2}+\mathrm{Cl}^{-}$ & $2.50 \cdot 10^{6}$ & & Frenzel et al. ${ }^{126}$ \\
\hline A62 & $\mathrm{ClNO}_{2}+\mathrm{NO}_{2}^{-} \rightarrow \mathrm{N}_{2} \mathrm{O}_{4}+\mathrm{Cl}^{-}$ & $7.97 \cdot 10^{3}$ & & Lahoutifard et al. ${ }^{127}$, average \\
\hline A63 & $\mathrm{Cl}+\mathrm{NO}_{2}^{-} \rightarrow \mathrm{Cl}^{-}+\mathrm{NO}_{2}$ & $5.00 \cdot 10^{9}$ & & Buxton et al. ${ }^{128}$ \\
\hline A64 & $\mathrm{Cl}+\mathrm{HOCl} \rightarrow \mathrm{Cl}^{-}+\mathrm{ClO}+\mathrm{H}^{+}$ & $3.00 \cdot 10^{9}$ & & Klaning and Wolff ${ }^{129}$ \\
\hline A65 & $\mathrm{Cl}+\mathrm{ClO}^{-} \rightarrow \mathrm{Cl}^{-}+\mathrm{ClO}$ & $8.20 \cdot 10^{9}$ & & Klaning and Wolff ${ }^{129}$ \\
\hline A66 & $\mathrm{ClO}^{-}+\mathrm{SO}_{3}{ }^{2-} \rightarrow \mathrm{Cl}^{-}+\mathrm{SO}_{4}{ }^{2-}$ & $2.30 \cdot 10^{4}$ & & Furrow et al. 130 \\
\hline A67 & $\mathrm{HOCl}+\mathrm{SO}_{4}^{-} \rightarrow \mathrm{ClO}+\mathrm{HSO}_{4}^{-}$ & $1.10 \cdot 10^{7}$ & & Zuo et al. ${ }^{131}$ \\
\hline A68 & $\mathrm{ClO}^{-}+\mathrm{SO}_{4}^{-} \rightarrow \mathrm{ClO}+\mathrm{SO}_{4}^{2-}$ & $1.30 \cdot 10^{9}$ & & Zuo et al. ${ }^{131}$ \\
\hline A69 & $\mathrm{ClO}_{2}^{-}+\mathrm{SO}_{4}^{-} \rightarrow \mathrm{ClO}_{2}+\mathrm{SO}_{4}{ }^{2-}$ & $2.05 \cdot 10^{9}$ & & Zuo et al. ${ }^{131}$ \\
\hline A70 & $\mathrm{HOCl}+\mathrm{NO}_{3} \rightarrow \mathrm{ClO}+\mathrm{HNO}_{3}$ & $4.30 \cdot 10^{7}$ & & Zuo et al. 131 \\
\hline A71 & $\mathrm{ClO}^{-}+\mathrm{NO}_{3} \rightarrow \mathrm{ClO}+\mathrm{NO}_{3}^{-}$ & $3.60 \cdot 10^{9}$ & & Zuo et al. ${ }^{131}$ \\
\hline A72 & $\mathrm{ClO}_{2}^{-}+\mathrm{NO}_{3} \rightarrow \mathrm{ClO}_{2}+\mathrm{NO}_{3}^{-}$ & $4.10 \cdot 10^{9}$ & & Zuo et al. ${ }^{131}$ \\
\hline A73 & $\mathrm{ClO}_{2}^{-}+\mathrm{HSO}_{3}^{-} \rightarrow \mathrm{OCl}^{-}+\mathrm{HSO}_{4}^{-}$ & $5.50 \cdot 10^{0}$ & & Hartz et al. ${ }^{132}$ \\
\hline A74 & $\mathrm{ClO}_{2}^{-}+\mathrm{SO}_{2} \rightarrow \mathrm{ClO}^{-}+\mathrm{SO}_{3}$ & $6.26 \cdot 10^{6}$ & & Hartz et al. ${ }^{132}$ \\
\hline A75 & $2 \mathrm{ClO} \rightarrow \mathrm{Cl}_{2} \mathrm{O}_{2}$ & $2.50 \cdot 10^{9}$ & & Klaning et al. ${ }^{118}$ \\
\hline A76 & $\mathrm{HOCl}+\mathrm{HClO}_{2} \rightarrow \mathrm{Cl}_{2} \mathrm{O}_{2}+\mathrm{H}_{2} \mathrm{O}$ & $1.00 \cdot 10^{5}$ & & Gordon and Tachiyashiki ${ }^{133}$ \\
\hline A77 & $\mathrm{HOCl}+\mathrm{ClO}_{2}^{-}+\mathrm{H}^{+} \rightarrow \mathrm{Cl}_{2} \mathrm{O}_{2}+\mathrm{H}_{2} \mathrm{O}$ & $1.06 \cdot 10^{6}$ & & Peintler et al. ${ }^{134}$ \\
\hline A78 & $\mathrm{Cl}_{2} \mathrm{O}_{2}+\mathrm{H}_{2} \mathrm{O} \rightarrow \mathrm{Cl}^{-}+\mathrm{ClO}_{3}^{-}+2 \mathrm{H}+$ & $1.80 \cdot 10^{2}$ & & Mialocq et al. ${ }^{135}$ \\
\hline A79 & $2 \mathrm{Cl}_{2} \mathrm{O}_{2} \rightarrow 2 \mathrm{ClO}_{2}+\mathrm{Cl}_{2}$ & $9.00 \cdot 10^{2}$ & & Sant'Anna et al. ${ }^{136}$ \\
\hline A 80 & $\mathrm{Cl}_{2} \mathrm{O}_{2}+\mathrm{ClO}_{2}^{-} \rightarrow \mathrm{Cl}^{-}+2 \mathrm{ClO}_{2}$ & $5.00 \cdot 10^{8}$ & & Peintler et al. ${ }^{134}$ \\
\hline A 81 & $\mathrm{OH}+\mathrm{ClO}_{3}^{-} \rightarrow \mathrm{ClO}_{3}+\mathrm{OH}^{-}$ & $1.00 \cdot 10^{6}$ & & Buxton and Subhani ${ }^{122}$ \\
\hline A 82 & $\mathrm{Cl}+\mathrm{ClO}_{3}^{-} \rightarrow \mathrm{ClO}_{3}+\mathrm{Cl}^{-}$ & $5.00 \cdot 10^{8}$ & & Buxton et al. ${ }^{128}$ \\
\hline A83 & $\mathrm{ClO}_{3} \rightarrow \mathrm{ClO}+\mathrm{O}_{2}$ & $2.00 \cdot 10^{5}$ & & estimated same as $\mathrm{BrO}_{3}$ \\
\hline A84 & $2 \mathrm{HOCl}+\mathrm{ClO}_{2}^{-} \rightarrow \mathrm{ClO}_{3}^{-}+\mathrm{Cl}_{2}+\mathrm{H}_{2} \mathrm{O}$ & $3.25 \cdot 10^{3}$ & & Peintler et al. ${ }^{134}$ \\
\hline A85 & $\mathrm{Cl}_{2}+\mathrm{H}_{2} \mathrm{O}_{2} \rightarrow 2 \mathrm{H}^{+}+2 \mathrm{Cl}^{-}+\mathrm{O}_{2}$ & $1.83 \cdot 10^{2}$ & 5387 & Connick ${ }^{112}$ \\
\hline A86 & $\mathrm{Cl}+\mathrm{Fe}^{2+} \rightarrow \mathrm{Cl}^{-}+\mathrm{Fe}^{3+}$ & $5.90 \cdot 10^{9}$ & & Jayson et al. ${ }^{137}$ \\
\hline A 87 & $\mathrm{FeCl}^{2+}+\mathrm{h} v \rightarrow \mathrm{Cl}+\mathrm{Fe}^{2+}$ & $7.59 \cdot 10^{-5}$ & & $\begin{array}{l}\text { Absorption David and David }{ }^{138} \text {, Quantum Yield } \\
\text { Nadtochenko and Kiwi }{ }^{139} \text {, TUV }\end{array}$ \\
\hline A88 & $\mathrm{Cl}^{-}+\mathrm{CH}_{3} \mathrm{CH}_{2} \mathrm{OOH} \rightarrow \mathrm{HOCl}+\mathrm{CH}_{3} \mathrm{COO}^{-}+\mathrm{H}_{2} \mathrm{O}$ & $1.47 \cdot 10^{-5}$ & & Shah et al. ${ }^{140}$ \\
\hline A89(2) & $\mathrm{Cl}_{2}^{-}+\mathrm{CH}_{3} \mathrm{OOH} \rightarrow 2 \mathrm{Cl}^{-}+\mathrm{H}^{+}+\mathrm{CH}_{3} \mathrm{OO}$ & $5.00 \cdot 10^{4}$ & 3340 & \\
\hline
\end{tabular}


(2) already implemented in CAPRAM-HM2.1, $\odot$ update of CAPRAM-HM2.1, new reactions in blue

\begin{tabular}{|c|c|c|c|}
\hline & Reaction & $\mathbf{k}_{\mathbf{2 9 8}}$ & $\mathbf{E}_{\mathrm{A}} / \mathbf{R} \quad$ Reference \\
\hline A90(2) & $\mathrm{Cl}+\mathrm{CH}_{3} \mathrm{OH} \rightarrow \mathrm{Cl}^{-}+\mathrm{H}^{+}+\mathrm{CH}_{2} \mathrm{OH}$ & $1.00 \cdot 10^{9}$ & 4089 \\
\hline A91 (2) & $\mathrm{Cl}_{2}^{-}+\mathrm{CH}_{3} \mathrm{OH} \rightarrow 2 \mathrm{Cl}^{-}+\mathrm{H}^{+}+\mathrm{CH}_{2} \mathrm{OH}$ & $5.10 \cdot 10^{4}$ & 5533 \\
\hline A92(2) & $\mathrm{Cl}+\mathrm{C}_{2} \mathrm{H}_{5} \mathrm{OH} \rightarrow \mathrm{Cl}^{-}+\mathrm{H}^{+}+\mathrm{CH}_{3} \mathrm{CHOH}$ & $1.60 \cdot 10^{9}$ & \\
\hline A93 (2) & $\mathrm{Cl}_{2}^{-}+\mathrm{C}_{2} \mathrm{H}_{5} \mathrm{OH} \rightarrow 2 \mathrm{Cl}^{-}+\mathrm{H}^{+}+\mathrm{CH}_{3} \mathrm{CHOH}$ & $1.20 \cdot 10^{5}$ & \\
\hline A94(2) & $\mathrm{Cl}+\mathrm{C}_{3} \mathrm{H}_{7} \mathrm{OH} \rightarrow \mathrm{Cl}^{-}+\mathrm{H}^{+}+\mathrm{C}_{2} \mathrm{H}_{5} \mathrm{CHOH}$ & $2.20 \cdot 10^{9}$ & 2285 \\
\hline A95 (2) & $\mathrm{Cl}_{2}^{-}+\mathrm{C}_{3} \mathrm{H}_{7} \mathrm{OH} \rightarrow 2 \mathrm{Cl}^{-}+\mathrm{H}^{+}+\mathrm{C}_{2} \mathrm{H}_{5} \mathrm{CHOH}$ & $1.00 \cdot 10^{5}$ & \\
\hline A96(2) & $\mathrm{Cl}+\mathrm{CH}_{3} \mathrm{CHOHCH}_{3} \rightarrow \mathrm{Cl}^{-}+\mathrm{H}^{+}+\mathrm{CH}_{3} \mathrm{COHCH}_{3}$ & $3.20 \cdot 10^{9}$ & 2766 \\
\hline A97(2) & $\mathrm{Cl}_{2}^{-}+\mathrm{CH}_{3} \mathrm{CHOHCH}_{3} \rightarrow 2 \mathrm{Cl}^{-}+\mathrm{H}^{+}+\mathrm{CH}_{3} \mathrm{COHCH}_{3}$ & $1.90 \cdot 10^{5}$ & \\
\hline A98(2) & $\mathrm{Cl}+\mathrm{CH}_{2}(\mathrm{OH})_{2} \rightarrow \mathrm{Cl}^{-}+\mathrm{H}^{+}+\mathrm{CH}(\mathrm{OH})_{2}$ & $1.40 \cdot 10^{9}$ & 3127 \\
\hline A99(2) & $\mathrm{Cl}_{2}^{-}+\mathrm{CH}_{2}(\mathrm{OH})_{2} \rightarrow 2 \mathrm{Cl}^{-}+\mathrm{H}^{+}+\mathrm{CH}(\mathrm{OH})_{2}$ & $3.60 \cdot 10^{4}$ & 4330 \\
\hline A100(2) & $\mathrm{Cl}+\mathrm{CH}_{3} \mathrm{CHO} \rightarrow \mathrm{Cl}^{-}+\mathrm{H}^{+}+\mathrm{CH}_{3} \mathrm{CO}$ & $6.00 \cdot 10^{8}$ & 1928 \\
\hline A101 (2) & $\mathrm{Cl}+\mathrm{CH}_{3} \mathrm{CH}(\mathrm{OH})_{2} \rightarrow \mathrm{Cl}^{-}+\mathrm{H}^{+}+\mathrm{CH}_{3} \mathrm{C}(\mathrm{OH})_{2}$ & $6.00 \cdot 10^{8}$ & 1928 \\
\hline A102(2) & $\mathrm{Cl}_{2}^{-}+\mathrm{CH}_{3} \mathrm{CHO} \rightarrow 2 \mathrm{Cl}^{-}+\mathrm{H}^{+}+\mathrm{CH}_{3} \mathrm{CO}$ & $4.00 \cdot 10^{4}$ & \\
\hline A103(2) & $\mathrm{Cl}_{2}^{-}+\mathrm{CH}_{3} \mathrm{CH}(\mathrm{OH})_{2} \rightarrow 2 \mathrm{Cl}^{-}+\mathrm{H}^{+}+\mathrm{CH}_{3} \mathrm{C}(\mathrm{OH})_{2}$ & $4.00 \cdot 10^{4}$ & \\
\hline A104(2) & $\mathrm{Cl}+\mathrm{C}_{2} \mathrm{H}_{5} \mathrm{CHO} \rightarrow \mathrm{Cl}^{-}+\mathrm{H}^{+}+\mathrm{C}_{2} \mathrm{H}_{5} \mathrm{CO}$ & $7.50 \cdot 10^{8}$ & 1566 \\
\hline A105 (2) & $\mathrm{Cl}+\mathrm{C}_{2} \mathrm{H}_{5} \mathrm{CH}(\mathrm{OH})_{2} \rightarrow \mathrm{Cl}^{-}+\mathrm{H}^{+}+\mathrm{C}_{2} \mathrm{H}_{5} \mathrm{C}(\mathrm{OH})_{2}$ & $7.50 \cdot 10^{8}$ & 1566 \\
\hline A106(2) & $\mathrm{Cl}+\mathrm{C}_{3} \mathrm{H}_{7} \mathrm{CHO} \rightarrow \mathrm{Cl}^{-}+\mathrm{H}^{+}+\mathrm{C}_{3} \mathrm{H}_{7} \mathrm{CO}$ & $2.20 \cdot 10^{9}$ & 1686 \\
\hline A107(2) & $\mathrm{Cl}+\mathrm{C}_{3} \mathrm{H}_{7} \mathrm{CH}(\mathrm{OH})_{2} \rightarrow \mathrm{Cl}^{-}+\mathrm{H}^{+}+\mathrm{C}_{3} \mathrm{H}_{7} \mathrm{C}(\mathrm{OH})_{2}$ & $1.10 \cdot 10^{9}$ & 1686 \\
\hline A108(2) & $\mathrm{Cl}+\mathrm{CH}_{3} \mathrm{COCH}_{3} \rightarrow \mathrm{Cl}^{-}+\mathrm{H}^{+}+\mathrm{CH}_{3} \mathrm{COCH}_{2}$ & $7.80 \cdot 10^{7}$ & \\
\hline A109(2) & $\mathrm{Cl}_{2}^{-}+\mathrm{CH}_{3} \mathrm{COCH}_{3} \rightarrow 2 \mathrm{Cl}^{-}+\mathrm{H}^{+}+\mathrm{CH}_{3} \mathrm{COCH}_{2}$ & $1.40 \cdot 10^{3}$ & \\
\hline A110 (2) & $\mathrm{Cl}+\mathrm{HCOOH} \rightarrow \mathrm{Cl}^{-}+\mathrm{H}^{+}+\mathrm{COOH}$ & $2.80 \cdot 10^{9}$ & 2405 \\
\hline A111(2) & $\mathrm{Cl}+\mathrm{HCOO}^{-} \rightarrow \mathrm{Cl}^{-}+\mathrm{COOH}$ & $4.20 \cdot 10^{9}$ & 1924 \\
\hline A112(2) & $\mathrm{Cl}_{2}^{-}+\mathrm{HCOOH} \rightarrow 2 \mathrm{Cl}^{-}+\mathrm{H}^{+}+\mathrm{COOH}$ & $8.00 \cdot 10^{4}$ & 4450 \\
\hline A113 (2) & $\mathrm{Cl}_{2}^{-}+\mathrm{HCOO}^{-} \rightarrow 2 \mathrm{Cl}^{-}+\mathrm{COOH}$ & $1.30 \cdot 10^{6}$ & 4930 \\
\hline A114(2) & $\mathrm{Cl}+\mathrm{CH}_{3} \mathrm{COOH} \rightarrow \mathrm{Cl}^{-}+\mathrm{H}^{+}+\mathrm{CH}_{2} \mathrm{COOH}$ & $1.00 \cdot 10^{8}$ & 1684 \\
\hline A115(2) & $\mathrm{Cl}+\mathrm{CH}_{3} \mathrm{COO}^{-} \rightarrow \mathrm{Cl}^{-}+\mathrm{CH}_{3}+\mathrm{CO}_{2}$ & $3.70 \cdot 10^{9}$ & 4930 \\
\hline A116(2) & $\mathrm{Cl}_{2}^{-}+\mathrm{CH}_{3} \mathrm{COOH} \rightarrow 2 \mathrm{Cl}^{-}+\mathrm{H}^{+}+\mathrm{CH}_{2} \mathrm{COOH}$ & $1.50 \cdot 10^{3}$ & 4800 \\
\hline A117(2) & $\mathrm{Cl}_{2}^{-}+\mathrm{CH}_{3} \mathrm{COO}^{-} \rightarrow 2 \mathrm{Cl}^{-}+\mathrm{CH}_{3}+\mathrm{CO}_{2}$ & $2.60 \cdot 10^{5}$ & 5292 \\
\hline A118(2) & $\mathrm{Cl}+\mathrm{C}_{2} \mathrm{H}_{5} \mathrm{COOH} \rightarrow \mathrm{Cl}^{-}+\mathrm{H}^{+}+\mathrm{CH}_{3} \mathrm{CHCOOH}$ & $1.20 \cdot 10^{9}$ & 5292 \\
\hline A119(2) & $\mathrm{Cl}+\mathrm{C}_{2} \mathrm{H}_{5} \mathrm{COO}^{-} \rightarrow \mathrm{Cl}^{-}+\mathrm{CH}_{3} \mathrm{CHCOO}^{-}$ & $1.20 \cdot 10^{9}$ & \\
\hline A120(2) & $\mathrm{Cl}_{2}^{-}+\mathrm{HC}_{2} \mathrm{O}_{4}^{-} \rightarrow 2 \mathrm{Cl}^{-}+\mathrm{H}^{+}+\mathrm{C}_{2} \mathrm{O}_{4}^{-}$ & $1.30 \cdot 10^{6}$ & \\
\hline
\end{tabular}


(2) already implemented in CAPRAM-HM2.1, $\odot$ update of CAPRAM-HM2.1, new reactions in blue

\begin{tabular}{|c|c|c|c|c|}
\hline & Reaction & $\mathbf{k}_{\mathbf{2 9 8}}$ & $\mathbf{E}_{\mathrm{A}} / \mathbf{R}$ & Reference \\
\hline A121 (2) & $\mathrm{Cl}_{2}^{-}+\mathrm{C}_{2} \mathrm{O}_{4}{ }^{2-} \rightarrow 2 \mathrm{Cl}^{-}+\mathrm{C}_{2} \mathrm{O}_{4}^{-}$ & $4.00 \cdot 10^{6}$ & & \\
\hline $\mathrm{A} 122(2)$ & $\mathrm{Cl}_{2}^{-}+\mathrm{CH}(\mathrm{OH})_{2} \mathrm{CH}(\mathrm{OH})_{2} \rightarrow 2 \mathrm{Cl}^{-}+\mathrm{H}^{+}+\mathrm{C}(\mathrm{OH})_{2} \mathrm{CH}(\mathrm{OH})_{2}$ & $4.00 \cdot 10^{4}$ & & \\
\hline A123 (2) & $\mathrm{Cl}_{2}^{-}+\mathrm{CH}(\mathrm{OH})_{2} \mathrm{COOH} \rightarrow 2 \mathrm{Cl}^{-}+\mathrm{H}^{+}+\mathrm{C}(\mathrm{OH})_{2} \mathrm{COOH}$ & $4.00 \cdot 10^{4}$ & & \\
\hline A124(2) & $\mathrm{CH}_{2} \mathrm{ClC}(\mathrm{OH})_{2} \mathrm{O}_{2} \rightarrow \mathrm{CH}_{2} \mathrm{ClCOOH}+\mathrm{HO}_{2}$ & $1.00 \cdot 10^{3}$ & & \\
\hline A125 (2) & $\mathrm{CH}_{2} \mathrm{ClC}(\mathrm{OH})_{2} \mathrm{O}_{2} \rightarrow \mathrm{CH}_{2} \mathrm{ClCOO}^{-}+\mathrm{H}^{+}+\mathrm{O}_{2}^{-}$ & $1.00 \cdot 10^{5}$ & & \\
\hline A126(2) & $\mathrm{CH}_{3} \mathrm{COCClO}+\mathrm{H}_{2} \mathrm{O} \rightarrow \mathrm{CH}_{3} \mathrm{COCOOH}+\mathrm{H}^{+}+\mathrm{Cl}^{-}$ & $3.50 \cdot 10^{2}$ & & \\
\hline A127(2) & $\mathrm{CHOCl} \rightarrow \mathrm{CO}+\mathrm{H}^{+}+\mathrm{Cl}^{-}$ & $1.00 \cdot 10^{4}$ & & \\
\hline $\mathrm{A} 128(2)$ & $\mathrm{CHOCl}+\mathrm{OH}^{-} \rightarrow \mathrm{HCOO}^{-}+\mathrm{H}^{+}+\mathrm{Cl}^{-}$ & $2.50 \cdot 10^{4}$ & & \\
\hline A129(2) & $\mathrm{COCl}_{2}+\mathrm{H}_{2} \mathrm{O} \rightarrow \mathrm{ClCOOH}+\mathrm{H}^{+}+\mathrm{Cl}^{-}$ & $1.00 \cdot 10^{1}$ & & \\
\hline A130(2) & $\mathrm{COCl}_{2}+\mathrm{OH}^{-} \rightarrow \mathrm{ClCOOH}+\mathrm{Cl}^{-}$ & $2.80 \cdot 10^{4}$ & & \\
\hline A131 (2) & $\mathrm{ClCOOH} \rightarrow \mathrm{CO}_{2}+\mathrm{H}^{+}+\mathrm{Cl}^{-}$ & $1.00 \cdot 10^{5}$ & & \\
\hline A132 & $\mathrm{CH}_{3} \mathrm{CClO}+\mathrm{H}_{2} \mathrm{O} \rightarrow \mathrm{CH}_{3} \mathrm{COOH}+\mathrm{Cl}^{-}+\mathrm{H}^{+}$ & $3.50 \cdot 10^{2}$ & & Prager et al. ${ }^{141}$ \\
\hline A133 & $\mathrm{ClCH}_{2} \mathrm{CClO}+\mathrm{H}_{2} \mathrm{O} \rightarrow \mathrm{ClCH}_{2} \mathrm{COOH}+\mathrm{Cl}^{-}+\mathrm{H}^{+}$ & $5.50 \cdot 10^{0}$ & & Prager et al. ${ }^{141}$ \\
\hline A134 & $\mathrm{ClCH}_{2} \mathrm{CH}_{2} \mathrm{OH}+\mathrm{OH} \rightarrow \mathrm{ClCH}_{2} \mathrm{CH}(\mathrm{OH}) \mathrm{O}_{2}+\mathrm{H}_{2} \mathrm{O}$ & $8.36 \cdot 10^{8}$ & 1067 & Morozov et al. ${ }^{142}$ \\
\hline A135 & $\mathrm{ClCH}_{2} \mathrm{CH}(\mathrm{OH}) \mathrm{O}_{2} \rightarrow \mathrm{ClCH}_{2} \mathrm{CHO}$ & $2.00 \cdot 10^{2}$ & & Bräuer ${ }^{143}$ \\
\hline A136 & $2 \mathrm{ClCH}_{2} \mathrm{CH}(\mathrm{OH}) \mathrm{O}_{2} \rightarrow \mathrm{ClCH}_{2} \mathrm{COOH}+\mathrm{H}_{2} \mathrm{O}_{2}$ & $7.30 \cdot 10^{8}$ & & Bräuer ${ }^{143}$ \\
\hline A137(2) & $\mathrm{Br}+\mathrm{Br} \rightarrow \mathrm{Br}_{2}$ & $1.00 \cdot 10^{9}$ & & \\
\hline A1380 & $\mathrm{Br}_{2}^{-}+\mathrm{Br}_{2}^{-} \rightarrow \mathrm{Br}_{3}^{-}+\mathrm{Br}^{-}$ & $2.04 \cdot 10^{9}$ & & revised \\
\hline A139(2) & $\mathrm{Br}^{-}+\mathrm{O}_{3} \rightarrow \mathrm{BrO}^{-}+\mathrm{O}_{2}$ & $2.10 \cdot 10^{2}$ & 4450 & \\
\hline A140(2) & $\mathrm{Br}+\mathrm{HO}_{2} \rightarrow \mathrm{H}^{+}+\mathrm{Br}^{-}+\mathrm{O}_{2}$ & $1.60 \cdot 10^{8}$ & & \\
\hline A141 (2) & $\mathrm{Br}+\mathrm{H}_{2} \mathrm{O}_{2} \rightarrow \mathrm{H}^{+}+\mathrm{Br}^{-}+\mathrm{HO}_{2}$ & $4.00 \cdot 10^{9}$ & & \\
\hline A142(2) & $\mathrm{Br}_{2}+\mathrm{HO}_{2} \rightarrow \mathrm{H}^{+}+\mathrm{Br}^{-}+\mathrm{O}_{2}$ & $1.10 \cdot 10^{8}$ & & \\
\hline A143(2) & $\mathrm{Br}_{2}+\mathrm{O}_{2}^{-} \rightarrow \mathrm{Br}_{2}^{-}+\mathrm{O}_{2}$ & $5.60 \cdot 10^{9}$ & & \\
\hline A144(2) & $\mathrm{Br}_{2}+\mathrm{H}_{2} \mathrm{O}_{2} \rightarrow 2 \mathrm{H}^{+}+2 \mathrm{Br}^{-}+\mathrm{O}_{2}$ & $1.30 \cdot 10^{3}$ & & \\
\hline $\mathrm{A} 145$ (2) & $\mathrm{Br}_{2}^{-}+\mathrm{OH} \rightarrow \mathrm{Br}^{-}+\mathrm{HOBr}$ & $1.00 \cdot 10^{9}$ & & \\
\hline A146(2) & $\mathrm{Br}_{2}^{-}+\mathrm{OH}^{-} \rightarrow 2 \mathrm{Br}^{-}+\mathrm{OH}$ & $1.10 \cdot 10^{4}$ & & \\
\hline A147(2) & $\mathrm{Br}_{2}^{-} \mathrm{HO}_{2} \rightarrow 2 \mathrm{Br}^{-}+\mathrm{H}^{+}+\mathrm{O}_{2}$ & $4.40 \cdot 10^{9}$ & & \\
\hline A148(2) & $\mathrm{Br}_{2}^{-}+\mathrm{HO}_{2} \rightarrow \mathrm{Br}_{2}+\mathrm{H}_{2} \mathrm{O}_{2}-\mathrm{H}^{+}$ & $4.40 \cdot 10^{9}$ & & \\
\hline A149(2) & $\mathrm{Br}_{2}^{-}+\mathrm{O}_{2}^{-} \rightarrow 2 \mathrm{Br}^{-}+\mathrm{O}_{2}$ & $1.70 \cdot 10^{8}$ & & \\
\hline A150(2) & $\mathrm{Br}_{2}^{-}+\mathrm{H}_{2} \mathrm{O}_{2} \rightarrow 2 \mathrm{Br}^{-}+\mathrm{H}^{+}+\mathrm{HO}_{2}$ & $1.00 \cdot 10^{5}$ & & \\
\hline A151 (2) & $\mathrm{Br}_{3}^{-}+\mathrm{HO}_{2} \rightarrow \mathrm{Br}_{2}^{-}+\mathrm{H}^{+}+\mathrm{Br}^{-}+\mathrm{O}_{2}$ & $1.00 \cdot 10^{7}$ & & \\
\hline
\end{tabular}


(2) already implemented in CAPRAM-HM2.1, $\odot$ update of CAPRAM-HM2.1, new reactions in blue

\begin{tabular}{|c|c|c|c|c|}
\hline & Reaction & $\mathbf{k}_{298}$ & $\mathbf{E}_{\mathrm{A}} / \mathbf{R}$ & Reference \\
\hline A152(2) & $\mathrm{Br}_{3}^{-}+\mathrm{O}_{2}^{-} \rightarrow \mathrm{Br}_{2}^{-}+\mathrm{Br}^{-}+\mathrm{O}_{2}$ & $3.80 \cdot 10^{9}$ & & \\
\hline A153 (2) & $\mathrm{BrO}+\mathrm{BrO} \rightarrow \mathrm{BrO}_{2}^{-}+\mathrm{BrO}^{-}+2 \mathrm{H}^{+}-\mathrm{H}_{2} \mathrm{O}$ & $2.80 \cdot 10^{9}$ & & \\
\hline A154(2) & $\mathrm{BrO}_{2}^{-}+\mathrm{BrO} \rightarrow \mathrm{BrO}_{2}+\mathrm{BrO}^{-}$ & $4.00 \cdot 10^{8}$ & & \\
\hline A155(2) & $\mathrm{Br}_{2}^{-}+\mathrm{BrO}_{2}^{-} \rightarrow 2 \mathrm{Br}^{-}+\mathrm{BrO}_{2}$ & $8.00 \cdot 10^{7}$ & & \\
\hline A156(2) & $\mathrm{BrO}_{2}^{-}+\mathrm{OH} \rightarrow \mathrm{BrO}_{2}+\mathrm{OH}^{-}$ & $1.80 \cdot 10^{9}$ & & \\
\hline A157(2) & $\mathrm{HOBr}+\mathrm{OH} \rightarrow \mathrm{BrO}+\mathrm{H}_{2} \mathrm{O}$ & $2.00 \cdot 10^{9}$ & & \\
\hline A158(2) & $\mathrm{BrO}^{-}+\mathrm{OH} \rightarrow \mathrm{BrO}+\mathrm{OH}^{-}$ & $4.50 \cdot 10^{9}$ & & \\
\hline A159(2) & $\mathrm{HOBr}+\mathrm{HO}_{2} \rightarrow \mathrm{Br}+\mathrm{H}_{2} \mathrm{O}+\mathrm{O}_{2}$ & $1.00 \cdot 10^{9}$ & & \\
\hline $\mathrm{A} 1600$ & $\mathrm{HOBr}+\mathrm{O}_{2}^{-} \rightarrow \mathrm{BrOH}^{-}+\mathrm{O}_{2}$ & $3.50 \cdot 10^{9}$ & & product Candeias et al. ${ }^{144}$ \\
\hline A161 (2) & $\mathrm{BrO}^{-}+\mathrm{O}_{2}^{-} \rightarrow \mathrm{Br}+2 \mathrm{OH}^{-}+\mathrm{O}_{2}-\mathrm{H}_{2} \mathrm{O}$ & $2.00 \cdot 10^{8}$ & & \\
\hline A162(2) & $\mathrm{HOBr}+\mathrm{H}_{2} \mathrm{O}_{2} \rightarrow \mathrm{H}^{+}+\mathrm{Br}^{-}+\mathrm{H}_{2} \mathrm{O}+\mathrm{O}_{2}$ & $3.50 \cdot 10^{6}$ & & \\
\hline A163 (2) & $\mathrm{BrO}^{-}+\mathrm{H}_{2} \mathrm{O}_{2} \rightarrow \mathrm{Br}^{-}+\mathrm{H}_{2} \mathrm{O}+\mathrm{O}_{2}$ & $2.00 \cdot 10^{5}$ & & \\
\hline A164(2) & $\mathrm{Br}_{2}^{-}+\mathrm{HSO}_{3}^{-} \rightarrow 2 \mathrm{Br}^{-}+\mathrm{H}^{+}+\mathrm{SO}_{3}^{-}$ & $5.00 \cdot 10^{7}$ & 780 & \\
\hline A165(2) & $\mathrm{Br}_{2}^{-}+\mathrm{SO}_{3}^{2-} \rightarrow 2 \mathrm{Br}^{-}+\mathrm{SO}_{3}^{-}$ & $3.30 \cdot 10^{7}$ & 650 & \\
\hline A166(2) & $\mathrm{Br}^{-}+\mathrm{SO}_{4}^{-} \rightarrow \mathrm{Br}+\mathrm{SO}_{4}^{2-}$ & $2.10 \cdot 10^{9}$ & & \\
\hline A167(2) & $\mathrm{HOBr}+\mathrm{SO}_{3}{ }^{2-} \rightarrow \mathrm{Br}^{-}+\mathrm{HSO}_{4}^{-}$ & $5.00 \cdot 10^{9}$ & & \\
\hline A168(2) & $\mathrm{HOBr}+\mathrm{HSO}_{3}^{-} \rightarrow \mathrm{H}++\mathrm{Br}^{-}+\mathrm{HSO}_{4}^{-}$ & $5.00 \cdot 10^{9}$ & & \\
\hline A169(2) & $\mathrm{Br}^{-}+\mathrm{HSO}_{5}^{-} \rightarrow \mathrm{HOBr}+\mathrm{SO}_{4}^{2-}$ & $1.00 \cdot 10^{0}$ & 5338 & \\
\hline A170(2) & $\mathrm{Br}_{2}^{-}+\mathrm{CH}_{2} \mathrm{OHSO}_{3}^{-} \rightarrow 2 \mathrm{Br}^{-}+\mathrm{CH}_{2} \mathrm{OHSO}_{3}$ & $5.00 \cdot 10^{4}$ & & \\
\hline A171 (2) & $\mathrm{Br}^{-}+\mathrm{NO}_{3} \rightarrow \mathrm{Br}+\mathrm{NO}_{3}^{-}$ & $3.80 \cdot 10^{9}$ & & \\
\hline A172(2) & $\mathrm{Br}_{2}^{-}+\mathrm{NO}_{2}^{-} \rightarrow 2 \mathrm{Br}^{-}+\mathrm{NO}_{2}$ & $1.20 \cdot 10^{7}$ & 1720 & \\
\hline A173(2) & $\mathrm{Br}^{-}+\mathrm{NO}_{2}^{+} \rightarrow \mathrm{BrNO}_{2}$ & $1.00 \cdot 10^{10}$ & & \\
\hline A174(2) & $\mathrm{Br}_{2}^{-}+\mathrm{Fe}^{2+} \rightarrow 2 \mathrm{Br}^{-}+\mathrm{Fe}^{3+}$ & $3.60 \cdot 10^{6}$ & 3330 & \\
\hline A175 (2) & $\mathrm{MnBr}_{2}^{+} \rightarrow 2 \mathrm{Br}-+\mathrm{Mn}^{3+}$ & $2.20 \cdot 10^{5}$ & & \\
\hline A176(2) & $\mathrm{Br}_{2}^{-}+\mathrm{Mn}^{2+} \rightarrow \mathrm{MnBr}_{2}^{+}$ & $6.30 \cdot 10^{6}$ & 4330 & \\
\hline A177(2) & $\mathrm{MnBr}_{2}^{+} \rightarrow \mathrm{Br}_{2}^{-}+\mathrm{Mn}^{2+}$ & $3.00 \cdot 10^{5}$ & & \\
\hline A178(2) & $\mathrm{Br}_{2}^{-}+\mathrm{Cu}^{+} \rightarrow 2 \mathrm{Br}^{-}+\mathrm{Cu}^{2+}$ & $3.60 \cdot 10^{6}$ & & \\
\hline A179(2) & $\mathrm{Br}+\mathrm{HCO}_{3}^{-} \rightarrow \mathrm{H}^{+}+\mathrm{Br}^{-}+\mathrm{CO}_{3}^{-}$ & $1.00 \cdot 10^{6}$ & & \\
\hline A180(2) & $\mathrm{Br}_{2}^{-}+\mathrm{CO}_{3}^{2-} \rightarrow 2 \mathrm{Br}^{-}+\mathrm{CO}_{3}^{-}$ & $1.10 \cdot 10^{5}$ & & \\
\hline A181 (2) & $\mathrm{Br}_{2}^{-}+\mathrm{HCO}_{3}^{-} \rightarrow 2 \mathrm{Br}^{-}+\mathrm{H}^{+}+\mathrm{CO}_{3}^{-}$ & $1.10 \cdot 10^{5}$ & & \\
\hline A182(2) & $\mathrm{BrNO}_{3} \rightarrow \mathrm{HOBr}+\mathrm{HNO}_{3}$ & $1.00 \cdot 10^{9}$ & & \\
\hline
\end{tabular}


(2) already implemented in CAPRAM-HM2.1, $\odot$ update of CAPRAM-HM2.1, new reactions in blue

\begin{tabular}{|c|c|c|c|c|}
\hline & Reaction & $\mathbf{k}_{\mathbf{2 9 8}}$ & $\mathbf{E}_{\mathrm{A}} / \mathbf{R}$ & Reference \\
\hline A183 (2) & $\mathrm{BrNO}_{3}+\mathrm{H}^{+} \rightarrow \mathrm{HOBr}+\mathrm{NO}_{2}^{+}$ & $1.12 \cdot 10^{3}$ & 6200 & \\
\hline A184 & $\mathrm{Br}^{-}+\mathrm{H}_{2} \mathrm{O}_{2} \rightarrow \mathrm{BrO}^{-}+\mathrm{H}_{2} \mathrm{O}$ & $1.10 \cdot 10^{-1}$ & 6746 & Mohammad and Liebhafsky ${ }^{113}$ \\
\hline A185 & $\mathrm{Br}^{-}+\mathrm{H}_{2} \mathrm{O}_{2}+\mathrm{H}^{+} \rightarrow \mathrm{HOBr}+\mathrm{H}_{2} \mathrm{O}$ & $5.00 \cdot 10^{-5}$ & 10441 & Mohammad and Liebhafsky ${ }^{113}$ \\
\hline A186 & $\mathrm{BrO}+\mathrm{O}_{3}^{-} \rightarrow \mathrm{BrO}^{-}+\mathrm{O}_{3}$ & $1.50 \cdot 10^{9}$ & & Klaning et al. ${ }^{145}$ \\
\hline A187 & $\mathrm{HOBr}+\mathrm{O}_{3} \rightarrow \mathrm{BrO}_{2}^{-}+\mathrm{H}^{+}+\mathrm{O}_{2}$ & $1.30 \cdot 10^{-2}$ & & Haag and Hoigne ${ }^{117}$ \\
\hline A188 & $\mathrm{BrO}^{-}+\mathrm{O}_{3} \rightarrow 0.77 \mathrm{Br}^{-}+0.23 \mathrm{BrO}_{2}^{-}+1.77 \mathrm{O}_{2}$ & $4.30 \cdot 10^{2}$ & & Haag and Hoigne ${ }^{117}$ \\
\hline A189 & $\mathrm{BrO}_{2}+\mathrm{NO}_{2}^{-} \rightarrow \mathrm{BrO}_{2}^{-}+\mathrm{NO}_{2}$ & $2.00 \cdot 10^{6}$ & & Huie and Neta ${ }^{121}$ \\
\hline A190 & $\mathrm{BrO}_{2}+\mathrm{SO}_{3}{ }^{2-} \rightarrow \mathrm{BrO}_{2}^{-}+\mathrm{SO}_{3}^{-}$ & $9.50 \cdot 10^{8}$ & & Huie and Neta ${ }^{121}$ \\
\hline A191 & $\mathrm{BrO}_{3}^{-}+\mathrm{SO}_{4}^{-} \rightarrow \mathrm{BrO}_{3}+\mathrm{SO}_{4}^{2-}$ & $1.40 \cdot 10^{6}$ & & Zuo and Katsumura ${ }^{146}$ \\
\hline A192 & $\mathrm{BrO}_{3} \rightarrow \mathrm{BrO}+\mathrm{O}_{2}$ & $2.00 \cdot 10^{5}$ & & Zuo and Katsumura ${ }^{146}$ \\
\hline A193 & $\mathrm{BrNO}_{2}+\mathrm{NO}_{2}^{-} \rightarrow \mathrm{N}_{2} \mathrm{O}_{4}+\mathrm{Br}^{-}$ & $1.27 \cdot 10^{4}$ & & Frenzel et al. ${ }^{126}$ \\
\hline A194 & $\mathrm{Br}+\mathrm{O}_{3} \rightarrow \mathrm{BrO}+\mathrm{O}_{2}$ & $1.50 \cdot 10^{8}$ & & Von Gunten and Oliveras ${ }^{147}$ \\
\hline A195 & $\mathrm{BrO}^{-}+\mathrm{SO}_{3}^{2-} \rightarrow \mathrm{Br}^{-}+\mathrm{SO}_{4}^{2-}$ & $1.00 \cdot 10^{8}$ & & Troy and Margerum ${ }^{148}$ \\
\hline A196 & $2 \mathrm{HOBr}+\mathrm{CO}_{3}{ }^{2-} \rightarrow \mathrm{BrO}_{2}{ }^{-}+\mathrm{Br}^{-}+\mathrm{HCO}_{3}{ }^{-}+\mathrm{H}^{+}$ & $3.30 \cdot 10^{-1}$ & & Beckwith and Margerum ${ }^{149}$ \\
\hline A197 & $2 \mathrm{HOBr}+\mathrm{OH}^{-} \rightarrow \mathrm{BrO}_{2}^{-}+\mathrm{Br}^{-}+\mathrm{H}^{+}+\mathrm{H}_{2} \mathrm{O}$ & $1.50 \cdot 10^{1}$ & & Beckwith and Margerum ${ }^{149}$ \\
\hline A198 & $\mathrm{BrO}^{-}+\mathrm{CO}_{3}^{-} \rightarrow \mathrm{BrO}+\mathrm{CO}_{3}^{2-}$ & $4.30 \cdot 10^{7}$ & & Buxton and Dainton ${ }^{150}$ \\
\hline A199 & $\mathrm{BrO}^{-}+\mathrm{Br}_{2}^{-} \rightarrow \mathrm{BrO}+2 \mathrm{Br}^{-}$ & $8.00 \cdot 10^{7}$ & & Buxton and Dainton ${ }^{150}$ \\
\hline A200 & $\mathrm{BrO}_{2}^{-}+\mathrm{CO}_{3}^{-} \rightarrow \mathrm{BrO}_{2}+\mathrm{CO}_{3}^{2-}$ & $1.10 \cdot 10^{8}$ & & Buxton and Dainton ${ }^{150}$ \\
\hline A201 & $\mathrm{BrO}_{2}+\mathrm{OH} \rightarrow \mathrm{BrO}_{3}^{-}+\mathrm{H}^{+}$ & $2.00 \cdot 10^{9}$ & & Field et al. ${ }^{151}$ \\
\hline A202 & $\mathrm{BrO}_{2}+\mathrm{Mn}^{2+} \rightarrow \mathrm{BrO}_{2}^{-}+\mathrm{Mn}^{3+}$ & $1.50 \cdot 10^{6}$ & & Field et al. ${ }^{151}$ \\
\hline A203 & $\mathrm{Br}_{2} \mathrm{O}_{4}+\mathrm{Mn}^{2+} \rightarrow \mathrm{BrO}_{2}+\mathrm{BrO}_{2}^{-}+\mathrm{Mn}^{3+}$ & $1.00 \cdot 10^{8}$ & & Field et al. ${ }^{151}$ \\
\hline A204 & $\mathrm{BrO}_{3}^{-}+\mathrm{SO}_{2} \rightarrow \mathrm{BrO}_{2}^{-}+\mathrm{SO}_{4}^{2-}+2 \mathrm{H}^{+}$ & $8.50 \cdot 10^{0}$ & & Szirovicza and Boga ${ }^{152}$ \\
\hline A205 & $\mathrm{BrO}_{3}^{-}+\mathrm{HSO}_{3}^{-} \rightarrow \mathrm{BrO}_{2}^{-}+\mathrm{SO}_{4}^{2-}+\mathrm{H}^{+}$ & $2.70 \cdot 10^{-2}$ & & Szirovicza and Boga ${ }^{152}$ \\
\hline A206 & $\mathrm{HBrO}_{2}+\mathrm{BrO}_{2}^{-} \rightarrow \mathrm{HOBr}+\mathrm{BrO}_{3}^{-}$ & $3.91 \cdot 10^{1}$ & & Faria et al. ${ }^{153}$ \\
\hline A207 & $\mathrm{HOBr}+\mathrm{BrO}_{2}^{-} \rightarrow \mathrm{Br}^{-}+\mathrm{BrO}_{3}^{-}+\mathrm{H}^{+}$ & $8.00 \cdot 10^{-3}$ & & Beckwith and Margerum ${ }^{149}$ \\
\hline A208 & $\mathrm{BrO}_{3}^{-}+\mathrm{OH} \rightarrow \mathrm{BrO}_{3}+\mathrm{OH}^{-}$ & $5.00 \cdot 10^{6}$ & & Amichai et al. ${ }^{154}$ \\
\hline A209(2) & $\mathrm{Br}_{2}^{-}+\mathrm{Cl}_{2}^{-} \rightarrow \mathrm{Br}_{2}+2 \mathrm{Cl}^{-}$ & $4.00 \cdot 10^{9}$ & & \\
\hline $\mathrm{A} 210$ (2) & $\mathrm{Br}^{-}+\mathrm{HOCl}+\mathrm{H}^{+} \rightarrow \mathrm{BrCl}+\mathrm{H}_{2} \mathrm{O}$ & $1.30 \cdot 10^{6}$ & & \\
\hline A211 (2) & $\mathrm{Br}^{-}+\mathrm{ClO}^{-} \rightarrow \mathrm{BrCl}+\mathrm{OH}^{-}-\mathrm{H}^{+}$ & $3.65 \cdot 10^{10}$ & & \\
\hline $\mathrm{A} 212(2)$ & $\mathrm{Br}^{-}+\mathrm{ClNO}_{2} \rightarrow \mathrm{BrCl}+\mathrm{NO}_{2}^{-}$ & $5.00 \cdot 10^{6}$ & & \\
\hline A213 (2) & $\mathrm{BrNO}_{2}+\mathrm{Cl}^{-} \rightarrow \mathrm{BrCl}+\mathrm{NO}_{2}^{-}$ & $1.00 \cdot 10^{1}$ & & \\
\hline
\end{tabular}


(2) already implemented in CAPRAM-HM2.1, $\odot$ update of CAPRAM-HM2.1, new reactions in blue

\begin{tabular}{|c|c|c|c|c|}
\hline & Reaction & $\mathbf{k}_{\mathbf{2 9 8}}$ & $\mathbf{E}_{\mathrm{A}} / \mathbf{R}$ & Reference \\
\hline A214 & $\mathrm{ClO}_{2}^{-}+\mathrm{Br}_{2}^{-} \rightarrow 2 \mathrm{Br}^{-}+\mathrm{ClO}_{2}$ & $2.00 \cdot 10^{7}$ & & Huie and Neta ${ }^{121}$ \\
\hline \multirow[t]{2}{*}{ A 215} & $\mathrm{BrO}_{2}^{-}+\mathrm{HOCl} \rightarrow$ & $1.60 \cdot 10^{2}$ & & Nicoson et al. ${ }^{155}$ \\
\hline & $\begin{array}{l}0.85 \mathrm{ClO}_{3}^{-}+0.93 \mathrm{HOBr}+0.08 \mathrm{ClO}_{2}^{-}+0.07 \mathrm{BrO}_{3}^{-}+0.92 \mathrm{Cl}^{-} \\
+0.92 \mathrm{H}^{+}-0.85 \mathrm{HOCl}\end{array}$ & & & \\
\hline A216 & $\mathrm{BrOCl}(\mathrm{OH})_{2}+\mathrm{H}^{+} \rightarrow \mathrm{HOBr}+\mathrm{HClO}_{2}+\mathrm{H}^{+}$ & $5.35 \cdot 10^{2}$ & & Sant'Anna and Faria ${ }^{156}$ \\
\hline A 217 & $\mathrm{HClO}_{2}+\mathrm{Br}^{-} \rightarrow \mathrm{HOCl}+\mathrm{BrO}^{-}$ & $1.00 \cdot 10^{-3}$ & & Sant'Anna and Faria ${ }^{156}$ \\
\hline A218 & $\mathrm{Br}+\mathrm{ClO}_{2}^{-} \rightarrow \mathrm{ClO}_{2}+\mathrm{Br}^{-}$ & $2.30 \cdot 10^{8}$ & & Toth and Fabian ${ }^{157}$ \\
\hline A219 & $\mathrm{Br}_{2}^{-}+\mathrm{ClO}_{2}^{-} \rightarrow \mathrm{ClO}_{2}+2 \mathrm{Br}^{-}$ & $4.00 \cdot 10^{6}$ & & Toth and Fabian ${ }^{157}$ \\
\hline $\mathrm{A} 220$ & $\mathrm{HOCl}+\mathrm{Br}^{-}+\mathrm{ClCH}_{2} \mathrm{COOH} \rightarrow \mathrm{BrCl}+\mathrm{ClCH}_{2} \mathrm{COO}^{-}+\mathrm{H}_{2} \mathrm{O}$ & $6.11 \cdot 10^{4}$ & & Kumar and Margerum ${ }^{158}$ \\
\hline A221 & $\mathrm{HOCl}+\mathrm{Br}^{-}+\mathrm{CH}_{3} \mathrm{COOH} \rightarrow \mathrm{BrCl}+\mathrm{CH}_{3} \mathrm{COO}^{-}+\mathrm{H}_{2} \mathrm{O}$ & $2.09 \cdot 10^{4}$ & & Kumar and Margerum ${ }^{158}$ \\
\hline A222 & $\mathrm{HOCl}+\mathrm{Br}^{-} \rightarrow \mathrm{BrCl}+\mathrm{OH}^{-}$ & $1.55 \cdot 10^{3}$ & & Kumar and Margerum ${ }^{158}$ \\
\hline A223 & $\mathrm{ClO}^{-}+\mathrm{Br}^{-}+\mathrm{HCO}_{3}^{-} \rightarrow \mathrm{BrCl}+\mathrm{OH}^{-}+\mathrm{CO}_{3}^{2-}$ & $1.25 \cdot 10^{2}$ & & Kumar and Margerum ${ }^{158}$ \\
\hline $\mathrm{A} 224$ & $\mathrm{ClO}^{-}+\mathrm{Br}^{-} \rightarrow \mathrm{BrCl}+2 \mathrm{OH}^{-}-\mathrm{H}_{2} \mathrm{O}$ & $8.88 \cdot 10^{-4}$ & & Kumar and Margerum ${ }^{158}$ \\
\hline A225 & $\mathrm{ClO}_{2}+\mathrm{BrO}_{2}+\mathrm{H}_{2} \mathrm{O} \rightarrow \mathrm{BrO}_{2}^{-}+\mathrm{ClO}_{3}^{-}+2 \mathrm{H}^{+}$ & $2.00 \cdot 10^{4}$ & & Wang et al. ${ }^{159}$ \\
\hline A226 & $\mathrm{ClO}_{2}+\mathrm{BrO}_{2}+\mathrm{OH}^{-} \rightarrow \mathrm{BrO}_{2}^{-}+\mathrm{ClO}_{3}^{-}+\mathrm{H}^{+}$ & $1.25 \cdot 10^{8}$ & & Wang et al. ${ }^{159}$ \\
\hline A227 & $\mathrm{ClO}_{2}+\mathrm{BrO}_{2}+\mathrm{SO}_{4}{ }^{2-} \rightarrow \mathrm{BrO}_{2}^{-}+\mathrm{ClO}_{3}^{-}+\mathrm{SO}_{4}^{2-}+\mathrm{H}_{2} \mathrm{O}-2 \mathrm{OH}^{-}$ & $3.60 \cdot 10^{7}$ & & Wang et al. ${ }^{159}$ \\
\hline A228 & $\mathrm{ClO}_{2}+\mathrm{BrO}_{2}+\mathrm{CH}_{3} \mathrm{COO}^{-} \rightarrow \mathrm{BrO}_{2}^{-}+\mathrm{ClO}_{3}^{-}+\mathrm{CH}_{3} \mathrm{COO}^{-}+\mathrm{H}_{2} \mathrm{O}-2 \mathrm{OH}^{-}$ & $4.40 \cdot 10^{7}$ & & Wang et al. ${ }^{159}$ \\
\hline A229 & $\mathrm{ClO}_{2}+\mathrm{BrO}_{2}+\mathrm{Cl}^{-} \rightarrow \mathrm{BrO}_{2^{-}}+\mathrm{ClO}_{3}^{-}+\mathrm{Cl}^{-}+\mathrm{H}_{2} \mathrm{O}-2 \mathrm{OH}^{-}$ & $1.28 \cdot 10^{8}$ & & Wang et al. ${ }^{159}$ \\
\hline A230 & $\mathrm{ClO}_{2}+\mathrm{BrO}_{2}+\mathrm{CO}_{3}^{2-} \rightarrow \mathrm{BrO}_{2-}^{-}+\mathrm{ClO}_{3}^{-}+\mathrm{CO}_{3}^{2-}+\mathrm{H}_{2} \mathrm{O}-2 \mathrm{OH}^{-}$ & $1.49 \cdot 10^{8}$ & & Wang et al. ${ }^{159}$ \\
\hline A231 & $\mathrm{ClO}_{2}+\mathrm{BrO}_{2}+\mathrm{ClO}_{2}^{-} \rightarrow \mathrm{BrO}_{2}^{-}+\mathrm{ClO}_{3}^{-}+\mathrm{ClO}_{2}^{-}+\mathrm{H}_{2} \mathrm{O}-2 \mathrm{OH}^{-}$ & $2.20 \cdot 10^{8}$ & & Wang et al. ${ }^{159}$ \\
\hline A232 & $\mathrm{ClO}_{2}+\mathrm{BrO}_{2}+\mathrm{BrO}_{2}^{-} \rightarrow \mathrm{BrO}_{2}^{-}+\mathrm{ClO}_{3}^{-}+\mathrm{BrO}_{2}^{-}+\mathrm{H}_{2} \mathrm{O}-2 \mathrm{OH}^{-}$ & $2.30 \cdot 10^{8}$ & & Wang et al. ${ }^{159}$ \\
\hline A233 & $\mathrm{ClO}_{2}+\mathrm{BrO}_{2}+\mathrm{Br}^{-} \rightarrow \mathrm{BrO}_{2}^{-}+\mathrm{ClO}_{3}^{-}+\mathrm{Br}^{-}+\mathrm{H}_{2} \mathrm{O}-2 \mathrm{OH}^{-}$ & $2.89 \cdot 10^{8}$ & & Wang et al. ${ }^{159}$ \\
\hline A234 & $\mathrm{Br}^{-}+\mathrm{CH}_{3} \mathrm{CH}_{2} \mathrm{OOH} \rightarrow \mathrm{HOBr}+\mathrm{CH}_{3} \mathrm{COO}^{-}+\mathrm{H}_{2} \mathrm{O}$ & $2.40 \cdot 10^{-1}$ & & Shah et al. ${ }^{140}$ \\
\hline A235 (2) & $\mathrm{Br}_{2}^{-}+\mathrm{CH}_{3} \mathrm{OOH} \rightarrow 2 \mathrm{Br}^{-}+\mathrm{H}^{+}+\mathrm{CH}_{3} \mathrm{OO}$ & $1.00 \cdot 10^{5}$ & & \\
\hline A236(2) & $\mathrm{Br}+\mathrm{CH}_{3} \mathrm{OH} \rightarrow \mathrm{Br}^{-}+\mathrm{H}^{+}+\mathrm{CH}_{2} \mathrm{OH}$ & $4.10 \cdot 10^{4}$ & 3368 & \\
\hline A237(2) & $\mathrm{Br}_{2}^{-}+\mathrm{CH}_{3} \mathrm{OH} \rightarrow 2 \mathrm{Br}^{-}+\mathrm{H}^{+}+\mathrm{CH}_{2} \mathrm{OH}$ & $1.00 \cdot 10^{3}$ & & \\
\hline A238(2) & $\mathrm{Br}+\mathrm{C}_{2} \mathrm{H}_{5} \mathrm{OH} \rightarrow \mathrm{Br}^{-}+\mathrm{H}^{+}+\mathrm{CH}_{3} \mathrm{CHOH}$ & $8.20 \cdot 10^{5}$ & 2285 & \\
\hline A239(2) & $\mathrm{Br}_{2}^{-}+\mathrm{C}_{2} \mathrm{H}_{5} \mathrm{OH} \rightarrow 2 \mathrm{Br}^{-}+\mathrm{H}^{+}+\mathrm{CH}_{3} \mathrm{CHOH}$ & $3.80 \cdot 10^{3}$ & & \\
\hline A240 (2) & $\mathrm{Br}+\mathrm{C}_{3} \mathrm{H}_{7} \mathrm{OH} \rightarrow \mathrm{Br}^{-}+\mathrm{H}^{+}+\mathrm{C}_{2} \mathrm{H}_{5} \mathrm{CHOH}$ & $3.80 \cdot 10^{5}$ & 1564 & \\
\hline A241 (2) & $\mathrm{Br}+\mathrm{CH}_{3} \mathrm{CHOHCH}_{3} \rightarrow \mathrm{Br}^{-}+\mathrm{H}^{+}+\mathrm{CH}_{3} \mathrm{COHCH}_{3}$ & $1.80 \cdot 10^{6}$ & 3127 & \\
\hline A242(2) & $\mathrm{Br}+\mathrm{CH}_{2}(\mathrm{OH})_{2} \rightarrow \mathrm{Br}^{-}+\mathrm{H}^{+}+\mathrm{CH}(\mathrm{OH})_{2}$ & $3.00 \cdot 10^{5}$ & 3608 & \\
\hline
\end{tabular}


(2) already implemented in CAPRAM-HM2.1, $\odot$ update of CAPRAM-HM2.1, new reactions in blue

\begin{tabular}{|c|c|c|c|c|}
\hline & Reaction & $\mathbf{k}_{\mathbf{2 9 8}}$ & $\mathbf{E}_{\mathbf{A}} / \mathbf{R}$ & Reference \\
\hline A243 (2) & $\mathrm{Br}_{2}^{-}+\mathrm{CH}_{2}(\mathrm{OH})_{2} \rightarrow 2 \mathrm{Br}^{-}+\mathrm{H}^{+}+\mathrm{CH}(\mathrm{OH})_{2}$ & $3.00 \cdot 10^{3}$ & & \\
\hline A244(2) & $\mathrm{Br}+\mathrm{CH}_{3} \mathrm{CHO} \rightarrow \mathrm{Br}^{-}+\mathrm{H}^{+}+\mathrm{CH}_{2} \mathrm{CHO}$ & $1.75 \cdot 10^{7}$ & 1804 & \\
\hline A245 (2) & $\mathrm{Br}+\mathrm{CH}_{3} \mathrm{CH}(\mathrm{OH})_{2} \rightarrow \mathrm{Br}^{-}+\mathrm{H}^{+}+\mathrm{CH}_{3} \mathrm{C}(\mathrm{OH})_{2}$ & $1.75 \cdot 10^{7}$ & 1804 & \\
\hline A246(2) & $\mathrm{Br}_{2}^{-}+\mathrm{CH}_{3} \mathrm{CHO} \rightarrow 2 \mathrm{Br}^{-}+\mathrm{H}^{+}+\mathrm{CH}_{2} \mathrm{CHO}$ & $2.15 \cdot 10^{5}$ & 2526 & \\
\hline A247(2) & $\mathrm{Br}_{2}^{-}+\mathrm{CH}_{3} \mathrm{CH}(\mathrm{OH})_{2} \rightarrow 2 \mathrm{Br}^{-}+\mathrm{H}^{+}+\mathrm{CH}_{3} \mathrm{C}(\mathrm{OH})_{2}$ & $2.15 \cdot 10^{5}$ & 2526 & \\
\hline A248(2) & $\mathrm{Br}+\mathrm{C}_{2} \mathrm{H}_{5} \mathrm{CHO} \rightarrow \mathrm{Br}^{-}+\mathrm{H}^{+}+\mathrm{C}_{2} \mathrm{H}_{5} \mathrm{CO}$ & $2.85 \cdot 10^{7}$ & 842 & \\
\hline A249(2) & $\mathrm{Br}+\mathrm{C}_{2} \mathrm{H}_{5} \mathrm{CH}(\mathrm{OH})_{2} \rightarrow \mathrm{Br}^{-}+\mathrm{H}^{+}+\mathrm{C}_{2} \mathrm{H}_{5} \mathrm{C}(\mathrm{OH})_{2}$ & $2.85 \cdot 10^{7}$ & 842 & \\
\hline A250(2) & $\mathrm{Br}_{2}^{-}+\mathrm{C}_{2} \mathrm{H}_{5} \mathrm{CHO} \rightarrow 2 \mathrm{Br}^{-}+\mathrm{H}^{+}+\mathrm{C}_{2} \mathrm{H}_{5} \mathrm{CO}$ & $4.95 \cdot 10^{5}$ & 3614 & \\
\hline A251 (2) & $\mathrm{Br}_{2}^{-}+\mathrm{C}_{2} \mathrm{H}_{5} \mathrm{CH}(\mathrm{OH})_{2} \rightarrow 2 \mathrm{Br}^{-}+\mathrm{H}^{+}+\mathrm{C}_{2} \mathrm{H}_{5} \mathrm{C}(\mathrm{OH})_{2}$ & $4.95 \cdot 10^{5}$ & 3614 & \\
\hline $\mathrm{A} 252(2)$ & $\mathrm{Br}+\mathrm{C}_{3} \mathrm{H}_{7} \mathrm{CHO} \rightarrow \mathrm{Br}^{-}+\mathrm{H}^{+}+\mathrm{C}_{3} \mathrm{H}_{7} \mathrm{CO}$ & $6.67 \cdot 10^{7}$ & 1203 & \\
\hline A253 (2) & $\mathrm{Br}+\mathrm{C}_{3} \mathrm{H}_{7} \mathrm{CH}(\mathrm{OH})_{2} \rightarrow \mathrm{Br}^{-}+\mathrm{H}^{+}+\mathrm{C}_{3} \mathrm{H}_{7} \mathrm{C}(\mathrm{OH})_{2}$ & $3.33 \cdot 10^{7}$ & 1203 & \\
\hline A254(2) & $\mathrm{Br}_{2}^{-}+\mathrm{C}_{3} \mathrm{H}_{7} \mathrm{CHO} \rightarrow 2 \mathrm{Br}^{-}+\mathrm{H}^{+}+\mathrm{C}_{3} \mathrm{H}_{7} \mathrm{CO}$ & $2.60 \cdot 10^{5}$ & 2289 & \\
\hline $\mathrm{A} 255$ (2) & $\mathrm{Br}_{2}^{-}+\mathrm{C}_{3} \mathrm{H}_{7} \mathrm{CH}(\mathrm{OH})_{2} \rightarrow 2 \mathrm{Br}^{-}+\mathrm{H}^{+}+\mathrm{C}_{3} \mathrm{H}_{7} \mathrm{C}(\mathrm{OH})_{2}$ & $1.30 \cdot 10^{5}$ & 2289 & \\
\hline A256(2) & $\mathrm{Br}+\mathrm{HCOOH} \rightarrow \mathrm{Br}^{-}+\mathrm{H}^{+}+\mathrm{COOH}$ & $7.70 \cdot 10^{5}$ & 2288 & \\
\hline A257(2) & $\mathrm{Br}+\mathrm{HCOO}^{-} \rightarrow \mathrm{Br}^{-}+\mathrm{COOH}$ & $4.60 \cdot 10^{8}$ & & \\
\hline A258(2) & $\mathrm{Br}_{2}^{-}+\mathrm{HCOOH} \rightarrow 2 \mathrm{Br}^{-}+\mathrm{H}^{+}+\mathrm{COOH}$ & $4.00 \cdot 10^{3}$ & & \\
\hline A259(2) & $\mathrm{Br}_{2}^{-}+\mathrm{HCOO}^{-} \rightarrow 2 \mathrm{Br}^{-}+\mathrm{COOH}$ & $4.90 \cdot 10^{3}$ & & \\
\hline A260 (2) & $\mathrm{Br}_{2}^{-}+\mathrm{CH}_{3} \mathrm{COOH} \rightarrow 2 \mathrm{Br}^{-}+\mathrm{H}^{+}+\mathrm{CH}_{2} \mathrm{COOH}$ & $1.00 \cdot 10^{1}$ & & \\
\hline A261 (2) & $\mathrm{Br}_{2}^{-}+\mathrm{CH}_{3} \mathrm{COO}^{-} \rightarrow 2 \mathrm{Br}^{-}+\mathrm{CH}_{3}+\mathrm{CO}_{2}$ & $1.00 \cdot 10^{2}$ & & \\
\hline A262(2) & $\mathrm{Br}_{2}^{-}+\mathrm{HC}_{2} \mathrm{O}_{4}^{-} \rightarrow 2 \mathrm{Br}^{-}+\mathrm{H}^{+}+\mathrm{C}_{2} \mathrm{O}_{4}^{-}$ & $3.70 \cdot 10^{3}$ & & \\
\hline A263 (2) & $\mathrm{Br}_{2}^{-}+\mathrm{C}_{2} \mathrm{O}_{4}^{2-} \rightarrow 2 \mathrm{Br}^{-}+\mathrm{C}_{2} \mathrm{O}_{4}^{-}$ & $1.10 \cdot 10^{4}$ & & \\
\hline A264(2) & $\mathrm{Br}_{2}^{-}+\mathrm{CH}(\mathrm{OH})_{2} \mathrm{CH}(\mathrm{OH})_{2} \rightarrow 2 \mathrm{Br}^{-}+\mathrm{H}^{+}+\mathrm{C}(\mathrm{OH})_{2} \mathrm{CH}(\mathrm{OH})_{2}$ & $5.00 \cdot 10^{2}$ & & \\
\hline $\mathrm{A} 265$ (2) & $\mathrm{Br}_{2}^{-}+\mathrm{CH}(\mathrm{OH})_{2} \mathrm{COOH} \rightarrow 2 \mathrm{Br}^{-}+\mathrm{H}^{+}+\mathrm{C}(\mathrm{OH})_{2} \mathrm{COOH}$ & $5.00 \cdot 10^{2}$ & & \\
\hline A266 & $\mathrm{HOBr}+\mathrm{HCOO}^{-} \rightarrow \mathrm{Br}^{-}+\mathrm{CO}_{2}+\mathrm{H}_{2} \mathrm{O}$ & $1.04 \cdot 10^{0}$ & & Brusa and Colussi ${ }^{160}$ \\
\hline A267 & $\mathrm{Br}_{2}+\mathrm{HCOO}^{-} \rightarrow 2 \mathrm{Br}^{-}+\mathrm{CO}_{2}+\mathrm{H}^{+}$ & $2.02 \cdot 10^{1}$ & & Brusa and Colussi ${ }^{160}$ \\
\hline A268 & $\mathrm{HOBr}+\mathrm{C}_{2} \mathrm{O}_{4} \mathrm{H}_{2} \rightarrow \mathrm{Br}^{-}+2 \mathrm{CO}_{2}+\mathrm{H}^{+}$ & $1.70 \cdot 10^{1}$ & & Pelle et al. ${ }^{161}$ \\
\hline A269 & $\mathrm{HBrO}_{2}+\mathrm{C}_{2} \mathrm{O}_{4} \mathrm{H}_{2} \rightarrow \mathrm{HOBr}+2 \mathrm{CO}_{2}+\mathrm{H}^{+}$ & $4.20 \cdot 10^{0}$ & & Pelle et al. ${ }^{161}$ \\
\hline $\mathrm{A} 270$ & $\mathrm{BrO}_{3}^{-}+\mathrm{C}_{2} \mathrm{O}_{4} \mathrm{H}_{2} \rightarrow \mathrm{BrO}_{2}^{-}+2 \mathrm{CO}_{2}+\mathrm{H}_{2} \mathrm{O}$ & $7.47 \cdot 10^{-4}$ & & Pelle et al. ${ }^{161}$ \\
\hline A271 (2) & $\mathrm{CH}_{2} \mathrm{BrC}(\mathrm{OH})_{2} \mathrm{O}_{2} \rightarrow \mathrm{CH}_{2} \mathrm{BrCOOH}+\mathrm{HO}_{2}$ & $1.00 \cdot 10^{3}$ & & \\
\hline A272(2) & $\mathrm{CH}_{2} \mathrm{BrC}(\mathrm{OH})_{2} \mathrm{O}_{2} \rightarrow \mathrm{CH}_{2} \mathrm{BrCOO}^{-}+\mathrm{H}^{+}+\mathrm{O}_{2}^{-}$ & $1.00 \cdot 10^{5}$ & & \\
\hline A273 (2) & $\mathrm{CH}_{3} \mathrm{COCBrO}+\mathrm{H}_{2} \mathrm{O} \rightarrow \mathrm{CH}_{3} \mathrm{COCOOH}+\mathrm{H}^{+}+\mathrm{Br}^{-}$ & $3.50 \cdot 10^{2}$ & & \\
\hline
\end{tabular}


(2) already implemented in CAPRAM-HM2.1, $Q$ update of CAPRAM-HM2.1, new reactions in blue

\begin{tabular}{|c|c|c|c|c|}
\hline & Reaction & $\mathbf{k}_{298}$ & $\overline{\mathbf{E}_{\mathrm{A}} / \mathbf{R}}$ & Reference \\
\hline A274(2) & $\mathrm{CHOBr} \rightarrow \mathrm{CO}+\mathrm{H}^{+}+\mathrm{Br}^{-}$ & $1.00 \cdot 10^{4}$ & & \\
\hline $\mathrm{A} 275$ (2) & $\mathrm{CHOBr}+\mathrm{OH}^{-} \rightarrow \mathrm{HCOO}^{-}+\mathrm{H}^{+}+\mathrm{Br}^{-}$ & $2.50 \cdot 10^{4}$ & & \\
\hline A276(2) & $\mathrm{COBr}_{2}+\mathrm{H}_{2} \mathrm{O} \rightarrow \mathrm{BrCOOH}+\mathrm{H}^{+}+\mathrm{Br}^{-}$ & $1.00 \cdot 10^{1}$ & & \\
\hline A277(2) & $\mathrm{COBr}_{2}+\mathrm{OH}^{-} \rightarrow \mathrm{BrCOOH}+\mathrm{Br}^{-}$ & $2.80 \cdot 10^{4}$ & & \\
\hline $\mathrm{A} 278(2)$ & $\mathrm{BrCOOH} \rightarrow \mathrm{CO}_{2}+\mathrm{H}^{+}+\mathrm{Br}^{-}$ & $1.00 \cdot 10^{5}$ & & \\
\hline A279(2) & $\mathrm{I}+\mathrm{I} \rightarrow \mathrm{I}_{2}$ & $1.10 \cdot 10^{10}$ & & \\
\hline $\mathrm{A} 280(2)$ & $\mathrm{I}+\mathrm{I}_{2}^{-} \rightarrow \mathrm{I}_{3}^{-}$ & $6.50 \cdot 10^{9}$ & & \\
\hline A281 (2) & $\mathrm{I}_{2}^{-}+\mathrm{I}_{2}^{-} \rightarrow \mathrm{I}_{3}^{-}+\mathrm{I}_{-}^{-}$ & $2.50 \cdot 10^{9}$ & & \\
\hline A282(2) & $\mathrm{I}^{-}+\mathrm{O}_{3} \rightarrow \mathrm{HOI}+\mathrm{O}_{2}$ & $2.17 \cdot 10^{9}$ & 8790 & \\
\hline A283 (2) & $\mathrm{I}_{2}+\mathrm{HO}_{2} \rightarrow \mathrm{I}_{2}^{-}+\mathrm{H}^{+}+\mathrm{O}_{2}$ & $6.00 \cdot 10^{9}$ & & \\
\hline A284(2) & $\mathrm{I}_{2}+\mathrm{O}_{2}^{-} \rightarrow \mathrm{I}_{2}^{-}+\mathrm{O}_{2}$ & $6.00 \cdot 10^{9}$ & & \\
\hline $\mathrm{A} 285$ (2) & $\mathrm{I}_{3}^{-}+\mathrm{HO}_{2} \rightarrow \mathrm{I}_{2}^{-}+\mathrm{H}^{+}+\mathrm{I}^{-}+\mathrm{O}_{2}$ & $2.50 \cdot 10^{8}$ & & \\
\hline A286(2) & $\mathrm{I}_{3}^{-}+\mathrm{O}_{2}^{-} \rightarrow \mathrm{I}_{2}^{-}+\mathrm{I}^{-}+\mathrm{O}_{2}$ & $2.50 \cdot 10^{8}$ & & \\
\hline A287(2) & $\mathrm{HIO}_{2}+\mathrm{H}_{2} \mathrm{O}_{2} \rightarrow \mathrm{H}^{+}+\mathrm{IO}_{3}^{-}+\mathrm{H}_{2} \mathrm{O}$ & $6.00 \cdot 10^{1}$ & & \\
\hline $\mathrm{A} 288(2)$ & $\mathrm{IO}_{2}^{-}+\mathrm{H}_{2} \mathrm{O}_{2} \rightarrow \mathrm{IO}_{3}^{-}+\mathrm{H}_{2} \mathrm{O}$ & $6.00 \cdot 10^{1}$ & & \\
\hline $\mathrm{A} 289$ (2) & $\mathrm{IO}+\mathrm{IO} \rightarrow \mathrm{HOI}+\mathrm{HIO}_{2}-\mathrm{H}_{2} \mathrm{O}$ & $1.50 \cdot 10^{9}$ & & \\
\hline A2900 & $\mathrm{I}_{2}+\mathrm{HSO}_{3}^{-} \rightarrow 2 \mathrm{H}^{+}+2 \mathrm{I}^{-}+\mathrm{HSO}_{4}^{-}-\mathrm{H}_{2} \mathrm{O}$ & $1.70 \cdot 10^{9}$ & & value from Yiin and Margerum ${ }^{162}$ \\
\hline A291 (2) & $\mathrm{HOI}+\mathrm{SO}_{3}{ }^{2-} \rightarrow \mathrm{I}^{-}+\mathrm{HSO}_{4}^{-}$ & $5.00 \cdot 10^{9}$ & & \\
\hline A292(2) & $\mathrm{HOI}+\mathrm{HSO}_{3}^{-} \rightarrow \mathrm{H}^{+}+\mathrm{I}^{-}+\mathrm{HSO}_{4}^{-}$ & $5.00 \cdot 10^{9}$ & & \\
\hline A293 (2) & $\mathrm{INO}_{3} \rightarrow \mathrm{HOI}+\mathrm{HNO}_{3}$ & $1.62 \cdot 10^{6}$ & 2800 & \\
\hline A294(2) & $\mathrm{INO}_{3}+\mathrm{H}^{+} \rightarrow \mathrm{HOI}+\mathrm{NO}_{2}^{+}$ & $1.12 \cdot 10^{3}$ & 6200 & \\
\hline A295 & $\mathrm{I}^{-}+\mathrm{H}_{2} \mathrm{O}_{2} \rightarrow \mathrm{IO}^{-}+\mathrm{H}_{2} \mathrm{O}$ & $1.40 \cdot 10^{-2}$ & 8407 & Mohammad and Liebhafsky ${ }^{113}$ \\
\hline A296 & $\mathrm{I}^{-}+\mathrm{H}_{2} \mathrm{O}_{2}+\mathrm{H}^{+} \rightarrow \mathrm{HOI}+\mathrm{H}_{2} \mathrm{O}$ & $9.95 \cdot 10^{0}$ & 5261 & Mohammad and Liebhafsky ${ }^{113}$ \\
\hline A297 & $\mathrm{I}_{3}{ }^{-}+\mathrm{HSO}_{3}^{-} \rightarrow \mathrm{HSO}_{4}^{-}+3 \mathrm{I}^{-}+3 \mathrm{H}^{+}-\mathrm{H}_{2} \mathrm{O}$ & $1.50 \cdot 10^{7}$ & & Yiin and Margerum ${ }^{162}$ \\
\hline A298 & $\mathrm{I}_{2}+\mathrm{SO}_{3}{ }^{2-} \rightarrow \mathrm{SO}_{4}{ }^{2-}+2 \mathrm{I}^{-}+2 \mathrm{H}^{+}-\mathrm{H}_{2} \mathrm{O}$ & $3.10 \cdot 10^{9}$ & & Yiin and Margerum ${ }^{162}$ \\
\hline A299 & $\mathrm{I}_{3}^{-}+\mathrm{SO}_{3}{ }^{2-} \rightarrow \mathrm{SO}_{4}^{2-}+3 \mathrm{I}^{-}+2 \mathrm{H}^{+}-\mathrm{H}_{2} \mathrm{O}$ & $2.90 \cdot 10^{8}$ & & Yiin and Margerum 162 \\
\hline $\mathrm{A} 300$ & $\mathrm{I}^{-}+\mathrm{OH} \rightarrow \mathrm{IOH}^{-}$ & $1.60 \cdot 10^{10}$ & & Mulazzani and Buxton ${ }^{163}$ \\
\hline A301 & $\mathrm{I}^{-}+\mathrm{NO}_{3} \rightarrow \mathrm{I}+\mathrm{NO}_{3}^{-}$ & $4.60 \cdot 10^{9}$ & & Rudich et al. 164 \\
\hline $\mathrm{A} 302$ & $\mathrm{HOI}+\mathrm{O}_{3} \rightarrow \mathrm{HIO}_{2}+\mathrm{O}_{2}$ & $3.60 \cdot 10^{4}$ & & Bichsel and von Gunten ${ }^{165}$ \\
\hline $\mathrm{A} 303$ & $\mathrm{IO}^{-}+\mathrm{O}_{3} \rightarrow \mathrm{IO}_{2}^{-}+\mathrm{O}_{2}$ & $1.60 \cdot 10^{6}$ & & Bichsel and von Gunten 165 \\
\hline A304 & $\mathrm{HOI}+\mathrm{OH} \rightarrow \mathrm{IO}+\mathrm{H}_{2} \mathrm{O}$ & $7.00 \cdot 10^{9}$ & & Buxton and Mulazzani ${ }^{166}$ \\
\hline
\end{tabular}


(2) already implemented in CAPRAM-HM2.1, $Q$ update of CAPRAM-HM2.1, new reactions in blue

\begin{tabular}{|c|c|c|c|c|}
\hline & Reaction & $\mathbf{k}_{\mathbf{2 9 8}}$ & $\mathbf{E}_{\mathrm{A}} / \mathbf{R}$ & Reference \\
\hline A305 & $\mathrm{I}_{2}^{-}+\mathrm{HOI} \rightarrow \mathrm{IO}+2 \mathrm{I}^{-}+\mathrm{H}+$ & $1.00 \cdot 10^{5}$ & & Buxton and Sellers ${ }^{167}$ \\
\hline A306 & $\mathrm{HOI}+\mathrm{H}_{2} \mathrm{O}_{2} \rightarrow \mathrm{I}^{-}+\mathrm{H}^{+}+\mathrm{O}_{2}+\mathrm{H}_{2} \mathrm{O}$ & $3.70 \cdot 10^{1}$ & & De Kepper and Epstein ${ }^{168}$ \\
\hline A307 & $\mathrm{I}_{2} \mathrm{O}_{2}+\mathrm{H}^{+} \rightarrow \mathrm{HIO}_{2}+\mathrm{HOI}+\mathrm{H}^{+}-\mathrm{H}_{2} \mathrm{O}$ & $3.20 \cdot 10^{4}$ & & Valkai and Horvath ${ }^{169}$ \\
\hline A308 & $2 \mathrm{IO}_{2}^{-} \rightarrow \mathrm{IO}_{3}^{-}+\mathrm{IO}^{-}$ & $1.03 \cdot 10^{2}$ & & Sebök-Nagy and Körtvélyesi ${ }^{170}$ \\
\hline A309 & $\mathrm{IO}_{3}^{-}+\mathrm{OH} \rightarrow \mathrm{IO}_{3}+\mathrm{OH}^{-}$ & $1.08 \cdot 10^{5}$ & & Mezyk ${ }^{171}$ \\
\hline A310 & $\mathrm{IO}_{3} \rightarrow \mathrm{IO}+\mathrm{O}_{2}$ & $2.00 \cdot 10^{5}$ & & estimated same as for $\mathrm{BrO}_{3}$ \\
\hline A311 & $\mathrm{IO}_{3}^{-}+\mathrm{HSO}_{3}^{-}+\mathrm{H}^{+} \rightarrow \mathrm{HIO}_{2}+\mathrm{SO}_{4}^{2-}+\mathrm{H}^{+}$ & $3.97 \cdot 10^{3}$ & & Cseko et al. ${ }^{172}$ \\
\hline A312 & $\mathrm{IO}_{3}^{-}+\mathrm{HSO}_{3}^{-} \rightarrow \mathrm{HIO}_{2}+\mathrm{SO}_{4}^{2-}$ & $1.46 \cdot 10^{-1}$ & & Cseko et al. ${ }^{172}$ \\
\hline A313(2) & $\mathrm{I}^{-}+\mathrm{ICl} \rightarrow \mathrm{I}_{2}+\mathrm{Cl}^{-}$ & $1.10 \cdot 10^{9}$ & & \\
\hline A314(2) & $\mathrm{I}^{-}+\mathrm{HOCl}+\mathrm{H}^{+} \rightarrow \mathrm{ICl}+\mathrm{H}_{2} \mathrm{O}$ & $3.50 \cdot 10^{11}$ & & \\
\hline A315 (2) & $\mathrm{I}^{-}+\mathrm{HOBr} \rightarrow \mathrm{IBr}+\mathrm{OH}^{-}$ & $5.00 \cdot 10^{9}$ & & \\
\hline A316(2) & $\mathrm{HOI}+\mathrm{Cl}_{2} \rightarrow \mathrm{HIO}_{2}+2 \mathrm{H}^{+}+2 \mathrm{Cl}^{-}-\mathrm{H}_{2} \mathrm{O}$ & $1.00 \cdot 10^{6}$ & & \\
\hline A317(2) & $\mathrm{HOI}+\mathrm{HOCl} \rightarrow \mathrm{HIO}_{2}+\mathrm{H}^{+}+\mathrm{Cl}^{-}$ & $5.00 \cdot 10^{5}$ & & \\
\hline A 3180 & $\mathrm{HOI}+\mathrm{HOBr} \rightarrow \mathrm{HIO}_{2}+\mathrm{H}^{+}+\mathrm{Br}^{-}$ & $1.00 \cdot 10^{0}$ & & revised, Criquet et al. ${ }^{173}$ \\
\hline A319(2) & $\mathrm{HIO}_{2}+\mathrm{HOCl} \rightarrow \mathrm{IO}_{3}^{-}+\mathrm{Cl}^{-}+2 \mathrm{H}^{+}$ & $1.50 \cdot 10^{3}$ & & \\
\hline A320 (2) & $\mathrm{IO}_{2}^{-}+\mathrm{HOCl} \rightarrow \mathrm{IO}_{3}^{-}+\mathrm{Cl}^{-}+\mathrm{H}^{+}$ & $1.50 \cdot 10^{3}$ & & \\
\hline A321 (2) & $\mathrm{HIO}_{2}+\mathrm{HOBr} \rightarrow \mathrm{IO}_{3}^{-}+\mathrm{Br}^{-}+2 \mathrm{H}^{+}$ & $1.00 \cdot 10^{6}$ & & \\
\hline A322(2) & $\mathrm{IO}_{2}^{-}+\mathrm{HOBr} \rightarrow \mathrm{IO}_{3}^{-}+\mathrm{Br}^{-}+\mathrm{H}^{+}$ & $1.00 \cdot 10^{6}$ & & \\
\hline A323 & $\mathrm{IO}^{-}+\mathrm{HOBr} \rightarrow \mathrm{IO}_{2}^{-}+\mathrm{H}^{+}+\mathrm{Br}^{-}$ & $1.90 \cdot 10^{6}$ & & Criquet et al. ${ }^{173}$ \\
\hline A324 & $\mathrm{IO}^{-}+\mathrm{BrO}^{-} \rightarrow \mathrm{IO}_{2}^{-}+\mathrm{Br}-$ & $1.80 \cdot 10^{3}$ & & Criquet et al. ${ }^{173}$ \\
\hline A 325 & $\mathrm{ClO}_{2}+\mathrm{I}^{-} \rightarrow \mathrm{ClO}_{2}^{-}+\mathrm{I}$ & $1.40 \cdot 10^{3}$ & & Hoigne and Bader ${ }^{115}$ \\
\hline A326 & $\mathrm{BrO}_{3}^{-}+\mathrm{I}^{-}+2 \mathrm{H}^{+} \rightarrow \mathrm{HBrO}_{2}+\mathrm{HOI}$ & $4.43 \cdot 10^{1}$ & & Simoyi et al. ${ }^{174}$ \\
\hline A 327 & $\mathrm{BrO}_{3}^{-}+\mathrm{IBr} \rightarrow \mathrm{IO}_{3}^{-}+\mathrm{Br}^{-}+\mathrm{HOBr}+\mathrm{H}^{+}$ & $8.00 \cdot 10^{-4}$ & & Chinake and Simoyi ${ }^{175}$ \\
\hline A328 & $\mathrm{HClO}_{2}+\mathrm{I}^{-} \rightarrow \mathrm{IO}^{-}+\mathrm{HOCl}$ & $5.00 \cdot 10^{0}$ & & Kern and $\mathrm{Kim}^{176}$ \\
\hline A329 & $\mathrm{HClO}_{2}+\mathrm{HOI} \rightarrow \mathrm{HIO}_{2}+\mathrm{HOCl}$ & $6.00 \cdot 10^{7}$ & & Kern and $\mathrm{Kim}^{176}$ \\
\hline A330 & $\mathrm{HBrO}_{2}+\mathrm{HOI} \rightarrow \mathrm{HIO}_{2}+\mathrm{HOBr}$ & $6.00 \cdot 10^{7}$ & & Faria et al. ${ }^{177}$, after $\mathrm{HClO}_{2}$ reaction rate \\
\hline A331 & $\mathrm{BrO}^{-}+\mathrm{I}^{-}+\mathrm{H}_{2} \mathrm{O} \rightarrow \mathrm{IBr}+2 \mathrm{OH}^{-}$ & $6.80 \cdot 10^{5}$ & & Troy and Margerum 148 \\
\hline A332 & $\mathrm{ICl}+\mathrm{OH}^{-} \rightarrow \mathrm{HOI}+\mathrm{Cl}^{-}$ & $4.50 \cdot 10^{9}$ & & Wang et al. ${ }^{178}$ \\
\hline A333 & $\mathrm{ICl}+\mathrm{CO}_{3}{ }^{2-} \rightarrow \mathrm{HOI}+\mathrm{Cl}^{-}+\mathrm{HCO}_{3}^{-}-\mathrm{H}_{2} \mathrm{O}$ & $1.40 \cdot 10^{9}$ & & Wang et al. ${ }^{178}$ \\
\hline A334 & $\mathrm{IBr}+\mathrm{OH}^{-} \rightarrow \mathrm{HOI}+\mathrm{Br}^{-}$ & $6.00 \cdot 10^{9}$ & & Troy et al. ${ }^{179}$ \\
\hline A335 & $\mathrm{IBr}+\mathrm{CO}_{3}{ }^{2-} \rightarrow \mathrm{HOI}+\mathrm{Br}^{-}+\mathrm{HCO}_{3}^{-}-\mathrm{H}_{2} \mathrm{O}$ & $3.50 \cdot 10^{9}$ & & Troy et al. ${ }^{179}$ \\
\hline
\end{tabular}


(2) already implemented in CAPRAM-HM2.1, $Q$ update of CAPRAM-HM2.1, new reactions in blue

\begin{tabular}{|c|c|c|c|c|}
\hline & Reaction & $\mathbf{k}_{298}$ & $\mathbf{E}_{\mathrm{A}} / \mathbf{R}$ & Reference \\
\hline A336 & $\mathrm{ClO}^{-}+\mathrm{I}^{-}+\mathrm{HCO}_{3}^{-} \rightarrow \mathrm{ICl}+2 \mathrm{OH}^{-}+\mathrm{HCO}_{3}^{-}-\mathrm{H}_{2} \mathrm{O}$ & $9.40 \cdot 10^{6}$ & & Kumar et al. ${ }^{180}$ \\
\hline A337 & $\mathrm{ClO}^{-}+\mathrm{HOI} \rightarrow \mathrm{HIO}_{2}+\mathrm{Cl}^{-}$ & $5.20 \cdot 10^{1}$ & & Bichsel and von Gunten ${ }^{165}$ \\
\hline A338 & $\mathrm{I}^{-}+\mathrm{CH}_{3} \mathrm{CH}_{2} \mathrm{OOH} \rightarrow \mathrm{HOI}+\mathrm{CH}_{3} \mathrm{COO}^{-}+\mathrm{H}_{2} \mathrm{O}$ & $4.22 \cdot 10^{2}$ & & Awad et al. ${ }^{181}$ \\
\hline $\mathrm{A} 339(2)$ & $\mathrm{CH}_{2} \mathrm{IC}(\mathrm{OH})_{2} \mathrm{O}_{2} \rightarrow \mathrm{CH}_{2} \mathrm{ICOOH}+\mathrm{HO}_{2}$ & $1.00 \cdot 10^{3}$ & & \\
\hline $\mathrm{A} 340(2)$ & $\mathrm{CH}_{2} \mathrm{IC}(\mathrm{OH})_{2} \mathrm{O}_{2} \rightarrow \mathrm{CH}_{2} \mathrm{ICOO}^{-}+\mathrm{H}^{+}+\mathrm{O}_{2}^{-}$ & $1.00 \cdot 10^{5}$ & & \\
\hline $\mathrm{A} 341$ (2) & $\mathrm{CHOI} \rightarrow \mathrm{CO}+\mathrm{H}^{+}+\mathrm{I}^{-}$ & $1.00 \cdot 10^{4}$ & & \\
\hline A342(2) & $\mathrm{CHOI}+\mathrm{OH}^{-} \rightarrow \mathrm{HCOO}^{-}+\mathrm{H}^{+}+\mathrm{I}^{-}$ & $2.50 \cdot 10^{4}$ & & \\
\hline $\mathrm{A} 343(2)$ & $\mathrm{COI}_{2}+\mathrm{H}_{2} \mathrm{O} \rightarrow \mathrm{ICOOH}+\mathrm{H}^{+}+\mathrm{I}^{-}$ & $1.00 \cdot 10^{1}$ & & \\
\hline $\mathrm{A} 344(2)$ & $\mathrm{COI}_{2}+\mathrm{OH}^{-} \rightarrow \mathrm{ICOOH}+\mathrm{I}^{-}$ & $2.80 \cdot 10^{4}$ & & \\
\hline $\mathrm{A} 345(2)$ & $\mathrm{ICOOH} \rightarrow \mathrm{CO}_{2}+\mathrm{H}^{+}+\mathrm{I}^{-}$ & $1.00 \cdot 10^{5}$ & & \\
\hline A346 & $\mathrm{HOI}+\mathrm{CH}_{2}=\mathrm{CHCOOH}+\mathrm{H}^{+} \rightarrow \mathrm{HOCH}_{2} \mathrm{CH}(\mathrm{I}) \mathrm{COOH}+\mathrm{H}^{+}$ & $8.07 \cdot 10^{2}$ & & Furrow et al. ${ }^{130}$ \\
\hline A347 & $\mathrm{I}_{2}+\mathrm{HOOCCH}_{2} \mathrm{COOH} \rightarrow \mathrm{HOOCCH}(\mathrm{I}) \mathrm{COOH}+\mathrm{I}^{-}+\mathrm{H}^{+}$ & $4.00 \cdot 10^{1}$ & & De Kepper and Epstein ${ }^{168}$ \\
\hline
\end{tabular}


Table S8 Reversible aqueous-phase reactions implemented within the CAPRAM-HM3.0.

\begin{tabular}{|c|c|c|c|c|c|c|c|c|c|}
\hline & Reaction & $\mathbf{K}$ & Ref. & $\mathbf{k}_{\mathrm{f}, 298}$ & $\mathbf{E}_{\mathrm{A}} / \mathbf{R}$ & Ref. & $\mathbf{k}_{\mathbf{b}, 298}$ & $\mathbf{E}_{\mathrm{A}} / \mathbf{R}$ & Ref. \\
\hline $\mathrm{E} 1(2)$ & $\mathrm{Cl}+\mathrm{Cl}^{-} \rightleftharpoons \mathrm{Cl}_{2}^{-}$ & $1.4 \cdot 10^{5}$ & & $8.50 \cdot 10^{9}$ & & & $6.00 \cdot 10^{4}$ & & \\
\hline E2(2) & $\mathrm{Cl}_{2}+\mathrm{Cl}^{-} \rightleftharpoons \mathrm{Cl}_{3}^{-}$ & $1.80 \cdot 10^{-1}$ & & $2.00 \cdot 10^{4}$ & & & $1.10 \cdot 10^{5}$ & & \\
\hline E3 (2) & $\mathrm{Cl}_{2}+\mathrm{H}_{2} \mathrm{O} \rightleftharpoons \mathrm{H}^{+}+\mathrm{Cl}^{-}+\mathrm{HOCl}$ & $1.90 \cdot 10^{-5} \mathrm{e}^{-4500 / \mathrm{T}}$ & & $4.00 \cdot 10^{-1}$ & 8000 & & $2.10 \cdot 10^{4}$ & 3500 & \\
\hline $\mathrm{E} 4(2)$ & $\mathrm{HCl} \rightleftharpoons \mathrm{H}^{+}+\mathrm{Cl}^{-}$ & $1.72 \cdot 10^{6} \mathrm{e}^{6890 / \mathrm{T}}$ & & $5.00 \cdot 10^{11}$ & -6890 & & $2.90 \cdot 10^{5}$ & & \\
\hline E5(2) & $\mathrm{HOCl} \rightleftharpoons \mathrm{H}^{+}+\mathrm{ClO}^{-}$ & $3.00 \cdot 10^{-8}$ & & $1.50 \cdot 10^{3}$ & & & $5.00 \cdot 10^{10}$ & & \\
\hline E6(2) & $\mathrm{Cl}^{-}+\mathrm{OH} \rightleftharpoons \mathrm{ClOH}^{-}$ & $7.00 \cdot 10^{-1}$ & & $4.30 \cdot 10^{9}$ & & & $6.10 \cdot 10^{9}$ & & \\
\hline E7(2) & $\mathrm{Cl}+\mathrm{OH}^{-} \rightleftharpoons \mathrm{ClOH}^{-}$ & $7.83 \cdot 10^{8}$ & & $1.80 \cdot 10^{10}$ & & & $2.30 \cdot 10^{1}$ & & \\
\hline $\mathrm{E} 8(2)$ & $\mathrm{ClOH}^{-}+\mathrm{H}^{+} \rightleftharpoons \mathrm{Cl}+\mathrm{H}_{2} \mathrm{O}$ & $5.10 \cdot 10^{6}$ & & $2.10 \cdot 10^{10}$ & & & $4.10 \cdot 10^{3}$ & & \\
\hline E9(2) & $\mathrm{ClOH}^{-}+\mathrm{Cl}^{-} \rightleftharpoons \mathrm{Cl}_{2}^{-}+\mathrm{OH}^{-}$ & $2.20 \cdot 10^{-4}$ & & $1.00 \cdot 10^{4}$ & & & $4.50 \cdot 10^{7}$ & & \\
\hline E10(2) & $\mathrm{Cl}^{-}+\mathrm{SO}_{4}^{-} \rightleftharpoons \mathrm{Cl}+\mathrm{SO}_{4}^{2-}$ & $1.20 \cdot 10^{0}$ & & $2.52 \cdot 10^{8}$ & & & $2.10 \cdot 10^{8}$ & & \\
\hline E11(2) & $\mathrm{Cl}^{-}+\mathrm{NO}_{3} \rightleftharpoons \mathrm{Cl}+\mathrm{NO}_{3}^{-}$ & $3.40 \cdot 10^{0} \mathrm{e}^{-4300 / \mathrm{T}}$ & & $3.40 \cdot 10^{8}$ & 4300 & & $1.00 \cdot 10^{8}$ & & \\
\hline E12(2) & $\mathrm{Cl}^{-}+\mathrm{Fe}^{3+} \rightleftharpoons \mathrm{FeCl}^{2+}$ & $1.39 \cdot 10^{0}$ & & $3.00 \cdot 10^{3}$ & & & $2.16 \cdot 10^{3}$ & & \\
\hline E13 & $\mathrm{HClO}_{2} \rightleftharpoons \mathrm{H}^{+}+\mathrm{ClO}_{2}^{-}$ & $1.91 \cdot 10^{-2}$ & 182 & $9.55 \cdot 10^{8}$ & & & $5.00 \cdot 10^{10}$ & & \\
\hline E14 & $\mathrm{ClO}_{2}^{-}+\mathrm{O}_{3} \rightleftharpoons \mathrm{O}_{3}^{-}+\mathrm{ClO}_{2}$ & $4.55 \cdot 10^{1}$ & & $8.19 \cdot 10^{6}$ & & 183 & $1.80 \cdot 10^{5}$ & & 118 \\
\hline E15 & $\mathrm{HOCl}+\mathrm{NO}_{2}^{-} \rightleftharpoons \mathrm{ClNO}_{2}+\mathrm{OH}^{-}$ & $3.97 \cdot 10^{-4}$ & 184 & $1.99 \cdot 10^{7}$ & & & $5.00 \cdot 10^{10}$ & & \\
\hline E16 & $\mathrm{Cl}_{2}+\mathrm{SO}_{4}^{2-} \rightleftharpoons \mathrm{Cl}^{-}+\mathrm{HOCl}+\mathrm{HSO}_{4}^{-}$ & $1.14 \cdot 10^{-3}$ & & $3.20 \cdot 10^{1}$ & & 185 & $2.80 \cdot 10^{3}$ & & 185 \\
\hline E17 & $\mathrm{Cl}_{2}+\mathrm{HCOO}^{-} \rightleftharpoons \mathrm{Cl}^{-}+\mathrm{HOCl}+\mathrm{HCOOH}$ & $1.00 \cdot 10^{3}$ & & $1.20 \cdot 10^{2}$ & & 185 & $1.20 \cdot 10^{-1}$ & & 185 \\
\hline E18 & $\mathrm{Cl}_{2}+\mathrm{CH}_{3} \mathrm{COO}^{-} \rightleftharpoons \mathrm{Cl}^{-}+\mathrm{HOCl}+\mathrm{CH}_{3} \mathrm{COOH}$ & $3.36 \cdot 10^{4}$ & & $9.40 \cdot 10^{2}$ & & 185 & $2.80 \cdot 10^{-2}$ & & 185 \\
\hline E19 & $\mathrm{Cl}_{2}+\mathrm{CH}_{2} \mathrm{ClCOO}^{-} \rightleftharpoons \mathrm{Cl}^{-}+\mathrm{HOCl}+\mathrm{CH}_{2} \mathrm{ClCOOH}$ & $3.34 \cdot 10^{2}$ & & $9.70 \cdot 10^{1}$ & & 185 & $2.90 \cdot 10^{-1}$ & & 185 \\
\hline E20 & $\mathrm{Cl}_{2}^{-}+\mathrm{OH}^{-} \rightleftharpoons \mathrm{Cl}^{-}+\mathrm{ClOH}^{-}$ & $4.50 \cdot 10^{3}$ & & $4.50 \cdot 10^{7}$ & & 186 & $1.00 \cdot 10^{4}$ & & 186 \\
\hline E21 & $\mathrm{ClO}_{3}^{-}+\mathrm{SO}_{4}^{-} \rightleftharpoons \mathrm{ClO}_{3}+\mathrm{SO}_{4}^{2-}$ & $8.00 \cdot 10^{0}$ & & $4.00 \cdot 10^{6}$ & & 187 & $5.00 \cdot 10^{5}$ & & 187 \\
\hline E22 & $\mathrm{ClO}_{3}^{-}+\mathrm{NO}_{3} \rightleftharpoons \mathrm{ClO}_{3}+\mathrm{NO}_{3}^{-}$ & $4.20 \cdot 10^{0}$ & & $3.49 \cdot 10^{3}$ & & 187 & $8.30 \cdot 10^{2}$ & & 187 \\
\hline E23 & $\mathrm{ClO}^{-}+\mathrm{ClO}_{2} \rightleftharpoons \mathrm{ClO}+\mathrm{ClO}_{2}^{-}$ & $2.53 \cdot 10^{-10} \mathrm{e}^{-7337 / \mathrm{T}}$ & & $9.11 \cdot 10^{-1}$ & 7337 & 188 & $3.60 \cdot 10^{9}$ & & 188 \\
\hline E24 & $\mathrm{Cl}^{-}+\mathrm{ClO}_{3}^{-} \rightleftharpoons \mathrm{Cl}_{2} \mathrm{O}_{3}^{2-}$ & $5.00 \cdot 10^{0}$ & & $2.00 \cdot 10^{0}$ & & 136 & $4.00 \cdot 10^{-1}$ & & 136 \\
\hline E25 & $\mathrm{Cl}_{2} \mathrm{O}_{3}^{2-}+2 \mathrm{H}^{+} \rightleftharpoons \mathrm{Cl}_{2} \mathrm{O}_{3} \mathrm{H}_{2}$ & $9.09 \cdot 10^{-4}$ & & $2.00 \cdot 10^{-4}$ & & 136 & $2.20 \cdot 10^{-1}$ & & 136 \\
\hline E26 & $\mathrm{Cl}_{2} \mathrm{O}_{3} \mathrm{H}_{2}+\mathrm{H}^{+} \rightleftharpoons \mathrm{Cl}_{2} \mathrm{O}_{2}+\mathrm{H}^{+}$ & $1.00 \cdot 10^{-1}$ & & $8.00 \cdot 10^{2}$ & & 136 & $8.00 \cdot 10^{3}$ & & 136 \\
\hline $\mathrm{E} 270$ & $\mathrm{Cl}^{-}+\mathrm{NO}_{2}^{+} \rightleftharpoons \mathrm{ClNO}_{2}$ & $1.44 \cdot 10^{8}$ & & $3.90 \cdot 10^{10}$ & & 189 & $2.70 \cdot 10^{2}$ & & 189 \\
\hline E28(2) & $\mathrm{CH}_{2} \mathrm{ClCO}_{3}+\mathrm{H}_{2} \mathrm{O} \rightleftharpoons \mathrm{CH}_{2} \mathrm{ClC}(\mathrm{OH})_{2} \mathrm{O}_{2}$ & $3.67 \cdot 10^{2}$ & & $1.10 \cdot 10^{7}$ & & & $3.00 \cdot 10^{4}$ & & \\
\hline E29(2) & $\mathrm{CH}_{2} \mathrm{ClCOOH} \rightleftharpoons \mathrm{CH}_{2} \mathrm{ClCOO}^{-}+\mathrm{H}^{+}$ & $1.75 \cdot 10^{-5} \mathrm{e}^{46 / \mathrm{T}}$ & & $8.75 \cdot 10^{5}$ & -46 & & $5.00 \cdot 10^{10}$ & & \\
\hline E30 (2) & $\mathrm{Br}+\mathrm{Br}^{-} \rightleftharpoons \mathrm{Br}_{2}^{-}$ & $6.32 \cdot 10^{5}$ & & $1.20 \cdot 10^{10}$ & & & $1.90 \cdot 10^{4}$ & & \\
\hline
\end{tabular}




\begin{tabular}{|c|c|c|c|c|c|c|c|c|c|}
\hline & Reaction & $\mathbf{K}$ & Ref. & $\mathbf{k}_{\mathbf{f}, 298}$ & $\mathbf{E}_{\mathrm{A}} / \mathbf{R}$ & Ref. & $\mathbf{k}_{\mathrm{b}, \mathbf{2 9 8}}$ & $\mathbf{E}_{\mathrm{A}} / \mathbf{R}$ & Ref. \\
\hline E31 (2) & $\mathrm{Br}_{2}+\mathrm{Br}^{-} \rightleftharpoons \mathrm{Br}_{3}^{-}$ & $1.75 \cdot 10^{1}$ & & $9.60 \cdot 10^{8}$ & & & $5.50 \cdot 10^{7}$ & & \\
\hline E32(2) & $\mathrm{Br}_{2}+\mathrm{H}_{2} \mathrm{O} \rightleftharpoons \mathrm{H}^{+}+\mathrm{Br}^{-}+\mathrm{HOBr}$ & $1.06 \cdot 10^{-10} \mathrm{e}^{-7500 / \mathrm{T}}$ & & $1.70 \cdot 10^{0}$ & 7500 & & $1.60 \cdot 10^{10}$ & & \\
\hline E33 (2) & $\mathrm{HBr} \rightleftharpoons \mathrm{H}^{+}+\mathrm{Br}^{-}$ & $1.00 \cdot 10^{9}$ & & $5.00 \cdot 10^{11}$ & & & $5.00 \cdot 10^{2}$ & & \\
\hline E34(2) & $\mathrm{HOBr} \rightleftharpoons \mathrm{H}^{+}+\mathrm{BrO}^{-}$ & $2.00 \cdot 10^{-9}$ & & $1.00 \cdot 10^{2}$ & & & $5.00 \cdot 10^{10}$ & & \\
\hline E35 (2) & $\mathrm{Br}^{-}+\mathrm{OH} \rightleftharpoons \mathrm{BrOH}^{-}$ & $3.33 \cdot 10^{2}$ & & $1.10 \cdot 10^{10}$ & & & $3.30 \cdot 10^{7}$ & & \\
\hline E36(2) & $\mathrm{Br}+\mathrm{OH}^{-} \rightleftharpoons \mathrm{BrOH}^{-}$ & $3.10 \cdot 10^{3}$ & & $1.30 \cdot 10^{10}$ & & & $4.20 \cdot 10^{6}$ & & \\
\hline E37(2) & $\mathrm{BrOH}^{-}+\mathrm{H}^{+} \rightleftharpoons \mathrm{Br}+\mathrm{H}_{2} \mathrm{O}$ & $1.80 \cdot 10^{12}$ & & $4.40 \cdot 10^{10}$ & & & $2.45 \cdot 10^{-2}$ & & \\
\hline E38(2) & $\mathrm{BrOH}^{-}+\mathrm{Br}^{-} \rightleftharpoons \mathrm{Br}_{2}^{-}+\mathrm{OH}^{-}$ & $7.00 \cdot 10^{1}$ & & $1.90 \cdot 10^{8}$ & & & $2.70 \cdot 10^{6}$ & & \\
\hline $\mathrm{E} 39$ (2) & $\mathrm{HOBr}+\mathrm{HOBr} \rightleftharpoons \mathrm{H}^{+}+\mathrm{Br}^{-}+\mathrm{HBrO}_{2}$ & $6.70 \cdot 10^{-12}$ & & $2.00 \cdot 10^{-5}$ & & & $3.00 \cdot 10^{6}$ & & \\
\hline $\mathrm{E} 40$ (2) & $\mathrm{HBrO}_{2} \rightleftharpoons \mathrm{H}^{+}+\mathrm{BrO}_{2}^{-}$ & $1.26 \cdot 10^{-5}$ & & $6.30 \cdot 10^{5}$ & & & $5.00 \cdot 10^{10}$ & & \\
\hline $\mathrm{E} 41(2)$ & $\mathrm{HOBr}+\mathrm{HBrO}_{2} \rightleftharpoons 2 \mathrm{H}^{+}+\mathrm{Br}^{-}+\mathrm{BrO}_{3}^{-}$ & $1.70 \cdot 10^{0}$ & & $3.20 \cdot 10^{0}$ & & & $2.00 \cdot 10^{0}$ & & \\
\hline $\mathrm{E} 42(2)$ & $\mathrm{HBrO}_{2}+\mathrm{HBrO}_{2} \rightleftharpoons \mathrm{HOBr}+\mathrm{H}^{+}+\mathrm{BrO}_{3}^{-}$ & $3.00 \cdot 10^{11}$ & & $3.00 \cdot 10^{3}$ & & & $1.00 \cdot 10^{-8}$ & & \\
\hline $\mathrm{E} 43(2)$ & $\mathrm{Br}_{2} \mathrm{O}_{4}+\mathrm{H}_{2} \mathrm{O} \rightleftharpoons \mathrm{H}^{+}+\mathrm{BrO}_{3}^{-}+\mathrm{HBrO}_{2}$ & $5.26 \cdot 10^{1}$ & & $2.20 \cdot 10^{3}$ & & & $4.20 \cdot 10^{1}$ & & \\
\hline $\mathrm{E} 44(2)$ & $\mathrm{Br}_{2} \mathrm{O}_{4} \rightleftharpoons 2 \mathrm{BrO}_{2}$ & $5.30 \cdot 10^{-5}$ & & $7.40 \cdot 10^{4}$ & & & $1.40 \cdot 10^{9}$ & & \\
\hline $\mathrm{E} 45$ (2) & $\mathrm{Br}^{-}+\mathrm{CO}_{3}^{-} \rightleftharpoons \mathrm{Br}+\mathrm{CO}_{3}{ }^{2-}$ & $5.00 \cdot 10^{-2}$ & & $1.00 \cdot 10^{5}$ & & & $2.00 \cdot 10^{6}$ & & \\
\hline E46 & $\mathrm{BrO}_{2}^{-}+\mathrm{O}_{3} \rightleftharpoons \mathrm{O}_{3}^{-}+\mathrm{BrO}_{2}$ & $1.78 \cdot 10^{-4}$ & & $8.90 \cdot 10^{4}$ & & 183 & $5.00 \cdot 10^{8}$ & & 145 \\
\hline E47 & $\mathrm{Br}_{2}+\mathrm{SO}_{4}^{2-}+\mathrm{H}_{2} \mathrm{O} \rightleftharpoons \mathrm{HOBr}+\mathrm{Br}^{-}+\mathrm{HSO}_{4}^{-}$ & $6.15 \cdot 10^{-6}$ & & $2.28 \cdot 10^{4}$ & & 190 & $3.70 \cdot 10^{9}$ & & 190 \\
\hline E48 & $\mathrm{BrO}^{-}+\mathrm{HCO}_{3}^{-} \rightleftharpoons \mathrm{HOBr}+\mathrm{CO}_{3}^{2-}$ & $1.30 \cdot 10^{-1}$ & & $3.90 \cdot 10^{7}$ & & 148 & $3.00 \cdot 10^{8}$ & & 148 \\
\hline E49 & $\mathrm{HOBr}+\mathrm{NO}_{2}^{-} \rightleftharpoons \mathrm{BrNO}_{2}+\mathrm{OH}^{-}$ & $9.86 \cdot 10^{-6}$ & 184 & $4.93 \cdot 10^{5}$ & & & $5.00 \cdot 10^{10}$ & & \\
\hline E50(2) & $\mathrm{BrCl} \rightleftharpoons \mathrm{HOBr}+\mathrm{H}^{+}+\mathrm{Cl}^{-}-\mathrm{H}_{2} \mathrm{O}$ & $1.80 \cdot 10^{-5}$ & & $1.00 \cdot 10^{5}$ & & & $5.60 \cdot 10^{9}$ & & \\
\hline E51 (2) & $\mathrm{BrCl}^{-} \rightleftharpoons \mathrm{Br}^{-}+\mathrm{Cl}$ & $1.60 \cdot 10^{-7}$ & & $1.90 \cdot 10^{3}$ & & & $1.20 \cdot 10^{10}$ & & \\
\hline E52(2) & $\mathrm{BrCl}^{-} \rightleftharpoons \mathrm{Br}+\mathrm{Cl}^{-}$ & $6.10 \cdot 10^{-4}$ & & $6.10 \cdot 10^{4}$ & & & $1.00 \cdot 10^{8}$ & & \\
\hline E53 (2) & $\mathrm{BrCl}^{-}+\mathrm{Br}^{-} \rightleftharpoons \mathrm{Br}_{2}^{-}+\mathrm{Cl}^{-}$ & $1.86 \cdot 10^{3}$ & & $8.00 \cdot 10^{9}$ & & & $4.30 \cdot 10^{6}$ & & \\
\hline E54(2) & $\mathrm{BrCl}^{-}+\mathrm{Cl}^{-} \rightleftharpoons \mathrm{Br}^{-}+\mathrm{Cl}_{2}^{-}$ & $2.75 \cdot 10^{-8}$ & & $1.10 \cdot 10^{2}$ & & & $4.00 \cdot 10^{9}$ & & \\
\hline E55(2) & $\mathrm{Br}_{2} \mathrm{Cl}^{-} \rightleftharpoons \mathrm{BrCl}+\mathrm{Br}^{-}$ & $5.60 \cdot 10^{-5}$ & & $4.30 \cdot 10^{5}$ & & & $7.70 \cdot 10^{9}$ & & \\
\hline $\mathrm{E} 56(2)$ & $\mathrm{Br}_{2} \mathrm{Cl}^{-} \rightleftharpoons \mathrm{Br}_{2}+\mathrm{Cl}^{-}$ & $7.60 \cdot 10^{-1}$ & & $3.80 \cdot 10^{4}$ & & & $5.00 \cdot 10^{4}$ & & \\
\hline E57(2) & $\mathrm{BrCl}_{2}^{-} \rightleftharpoons \mathrm{BrCl}+\mathrm{Cl}^{-}$ & $1.70 \cdot 10^{-1}$ & & $1.70 \cdot 10^{5}$ & & & $1.00 \cdot 10^{6}$ & & \\
\hline E58(2) & $\mathrm{BrCl}_{2}^{-} \rightleftharpoons \mathrm{Br}^{-}+\mathrm{Cl}_{2}$ & $1.50 \cdot 10^{-6}$ & & $9.00 \cdot 10^{3}$ & & & $6.00 \cdot 10^{9}$ & & \\
\hline E59(2) & $\mathrm{Br}^{-}+\mathrm{ClOH}^{-} \rightleftharpoons \mathrm{BrCl}^{-}+\mathrm{OH}^{-}$ & $3.33 \cdot 10^{2}$ & & $1.00 \cdot 10^{9}$ & & & $3.00 \cdot 10^{6}$ & & \\
\hline E60(2) & $\mathrm{BrOH}^{-}+\mathrm{Cl}^{-} \rightleftharpoons \mathrm{BrCl}^{-}+\mathrm{OH}^{-}$ & $9.50 \cdot 10^{1}$ & & $1.90 \cdot 10^{8}$ & & & $2.00 \cdot 10^{7}$ & & \\
\hline E61 & $\mathrm{BrO}_{2}+\mathrm{ClO}_{2}^{-} \rightleftharpoons \mathrm{BrO}_{2}^{-}+\mathrm{ClO}_{2}$ & $1.00 \cdot 10^{6}$ & & $3.60 \cdot 10^{7}$ & & 121 & $3.60 \cdot 10^{1}$ & & 159 \\
\hline E62 & $\mathrm{BrCl}+\mathrm{BrCl} \rightleftharpoons \mathrm{Cl}_{2}+\mathrm{Br}_{2}$ & $7.60 \cdot 10^{-3}$ & 191 & $3.80 \cdot 10^{8}$ & & & $5.00 \cdot 10^{10}$ & & \\
\hline
\end{tabular}




\begin{tabular}{|c|c|c|c|c|c|c|c|c|c|}
\hline & Reaction & $\mathbf{K}$ & Ref. & $\mathbf{k}_{\mathrm{f}, 298}$ & $\mathbf{E}_{\mathrm{A}} / \mathbf{R}$ & Ref. & $k_{b, 298}$ & $\mathbf{E}_{\mathrm{A}} / \mathbf{R}$ & Ref. \\
\hline E63 & $\mathrm{BrO}^{-}+\mathrm{ClO}_{2} \rightleftharpoons \mathrm{BrO}+\mathrm{ClO}_{2}^{-}$ & $1.28 \cdot 10^{-7} \mathrm{e}^{-6615 / \mathrm{T}}$ & & $2.05 \cdot 10^{0}$ & 6615 & 188 & $1.60 \cdot 10^{7}$ & & 188 \\
\hline E64 & $\mathrm{Br}^{-}+\mathrm{ClO}_{3}^{-} \rightleftharpoons \mathrm{BrOClO}_{2}^{2-}$ & $5.41 \cdot 10^{-4}$ & & $2.00 \cdot 10^{0}$ & & 156 & $3.70 \cdot 10^{3}$ & & 156 \\
\hline E65 & $\mathrm{BrOClO}_{2}^{2-}+2 \mathrm{H}^{+} \rightleftharpoons \operatorname{BrOCl}(\mathrm{OH})_{2}$ & $4.00 \cdot 10^{-5}$ & & $1.00 \cdot 10^{-1}$ & & 156 & $2.50 \cdot 10^{3}$ & & 156 \\
\hline E66 & $\mathrm{Br}_{2}+\mathrm{ClO}_{2}^{-} \rightleftharpoons \mathrm{ClO}_{2}+\mathrm{Br}_{2}^{-}$ & $1.18 \cdot 10^{-6}$ & & $1.30 \cdot 10^{3}$ & & 157 & $1.10 \cdot 10^{9}$ & & 157 \\
\hline E670 & $\mathrm{Br}^{-}+\mathrm{BrNO}_{2} \rightleftharpoons \mathrm{Br}_{2}+\mathrm{NO}_{2}^{-}$ & $2.60 \cdot 10^{0}$ & & $1.85 \cdot 10^{6}$ & & 126 & $7.11 \cdot 10^{5}$ & & 126 \\
\hline E68(2) & $\mathrm{CH}_{2} \mathrm{BrCO}_{3}+\mathrm{H}_{2} \mathrm{O} \rightleftharpoons \mathrm{CH}_{2} \mathrm{BrC}(\mathrm{OH})_{2} \mathrm{O}_{2}$ & $3.67 \cdot 10^{2}$ & & $1.10 \cdot 10^{7}$ & & & $3.00 \cdot 10^{4}$ & & \\
\hline E69(2) & $\mathrm{CH}_{2} \mathrm{BrCOOH} \rightleftharpoons \mathrm{CH}_{2} \mathrm{BrCOO}^{-}+\mathrm{H}^{+}$ & $1.75 \cdot 10^{-5} \mathrm{e}^{46 / \mathrm{T}}$ & & $8.75 \cdot 10^{5}$ & -46 & & $5.00 \cdot 10^{10}$ & & \\
\hline E70(2) & $\mathrm{I}+\mathrm{I}^{-} \rightleftharpoons \mathrm{I}_{2}^{-}$ & $1.36 \cdot 10^{5}$ & & $9.10 \cdot 10^{9}$ & & & $6.70 \cdot 10^{4}$ & & \\
\hline E71 (2) & $\mathrm{I}_{2}+\mathrm{I}^{-} \rightleftharpoons \mathrm{I}_{3}^{-}$ & $7.13 \cdot 10^{2}$ & & $6.20 \cdot 10^{9}$ & & & $8.70 \cdot 10^{6}$ & & \\
\hline E72(2) & $\mathrm{HI} \rightleftharpoons \mathrm{H}^{+}+\mathrm{I}^{-}$ & $3.20 \cdot 10^{9}$ & & $5.00 \cdot 10^{11}$ & & & $1.56 \cdot 10^{2}$ & & \\
\hline E73 (2) & $\mathrm{HOI} \rightleftharpoons \mathrm{H}^{+}+\mathrm{IO}^{-}$ & $3.16 \cdot 10^{-11}$ & & $1.58 \cdot 10^{0}$ & & & $5.00 \cdot 10^{10}$ & & \\
\hline E74(2) & $\mathrm{HOI}+\mathrm{H}^{+}+\mathrm{I}^{-} \rightleftharpoons \mathrm{I}_{2}+\mathrm{H}_{2} \mathrm{O}$ & $1.47 \cdot 10^{12}$ & & $4.40 \cdot 10^{12}$ & & & $3.00 \cdot 10^{0}$ & & \\
\hline E75 (2) & $\mathrm{HOI}+\mathrm{HOI} \rightleftharpoons \mathrm{HIO}_{2}+\mathrm{H}^{+}+\mathrm{I}^{-}$ & $1.25 \cdot 10^{-9}$ & & $2.50 \cdot 10^{1}$ & & & $2.00 \cdot 10^{10}$ & & \\
\hline $\mathrm{E} 76(2)$ & $\mathrm{HOI}+\mathrm{HOI} \rightleftharpoons \mathrm{IO}_{2}^{-}+2 \mathrm{H}^{+}+\mathrm{I}^{-}$ & $1.25 \cdot 10^{-9}$ & & $2.50 \cdot 10^{1}$ & & & $2.00 \cdot 10^{10}$ & & \\
\hline E77(2) & $\mathrm{HIO}_{2} \rightleftharpoons \mathrm{H}^{+}+\mathrm{IO}_{2}^{-}$ & $2.51 \cdot 10^{-2}$ & & $1.26 \cdot 10^{9}$ & & & $5.00 \cdot 10^{10}$ & & \\
\hline $\mathrm{E} 78(2)$ & $\mathrm{HIO}_{3} \rightleftharpoons \mathrm{H}^{+}+\mathrm{IO}_{3}^{-}$ & $1.70 \cdot 10^{-1}$ & & $8.50 \cdot 10^{9}$ & & & $5.00 \cdot 10^{10}$ & & \\
\hline E79(2) & $\mathrm{HIO}_{2}+\mathrm{HOI} \rightleftharpoons \mathrm{IO}_{3}{ }^{-}+\mathrm{I}^{-}+2 \mathrm{H}^{+}$ & $2.00 \cdot 10^{-1}$ & & $2.40 \cdot 10^{2}$ & & & $1.20 \cdot 10^{3}$ & & \\
\hline $\mathrm{E} 80$ (2) & $\mathrm{IO}_{2}^{-}+\mathrm{HOI} \rightleftharpoons \mathrm{IO}_{3}^{-}+\mathrm{I}^{-}+\mathrm{H}^{+}$ & $2.00 \cdot 10^{-1}$ & & $2.40 \cdot 10^{2}$ & & & $1.20 \cdot 10^{3}$ & & \\
\hline $\mathrm{E} 81(2)$ & $\mathrm{IO}_{2}^{-}+\mathrm{I}_{2} \rightleftharpoons \mathrm{IO}_{3}^{-}+2 \mathrm{I}^{-}+2 \mathrm{H}^{+}-\mathrm{H}_{2} \mathrm{O}$ & $1.30 \cdot 10^{-13}$ & & $5.50 \cdot 10^{-5}$ & & & $4.20 \cdot 10^{8}$ & & \\
\hline E82 & $\mathrm{I}^{-}+\mathrm{Fe}^{3+} \rightleftharpoons \mathrm{FeI}^{2+}$ & $1.00 \cdot 10^{-3}$ & & $6.30 \cdot 10^{-3}$ & & 192 & $6.30 \cdot 10^{0}$ & & 193 \\
\hline E83 & $\mathrm{HOI}^{-} \rightleftharpoons \mathrm{I}+\mathrm{OH}^{-}$ & $2.19 \cdot 10^{-1}$ & & $3.50 \cdot 10^{7}$ & & 163 & $1.60 \cdot 10^{8}$ & & 163 \\
\hline E84 & $\mathrm{I}_{2}+\mathrm{OH}^{-} \rightleftharpoons \mathrm{I}_{2} \mathrm{OH}^{-}$ & $5.00 \cdot 10^{0}$ & & $1.00 \cdot 10^{10}$ & & 166 & $2.00 \cdot 10^{9}$ & & 166 \\
\hline $\mathrm{E} 85$ & $\mathrm{I}_{2} \mathrm{OH}^{-} \rightleftharpoons \mathrm{HOI}+\mathrm{I}^{-}$ & $8.30 \cdot 10^{0}$ & & $2.49 \cdot 10^{9}$ & & 166 & $3.00 \cdot 10^{8}$ & & 166 \\
\hline E86(2) & $\mathrm{IBr}+\mathrm{I}^{-} \rightleftharpoons \mathrm{I}_{2}+\mathrm{Br}^{-}$ & $4.20 \cdot 10^{5}$ & & $2.00 \cdot 10^{9}$ & & & $4.74 \cdot 10^{3}$ & & \\
\hline $\mathrm{E} 87(2)$ & $\mathrm{HOI}+\mathrm{H}^{+}+\mathrm{Cl}^{-} \rightleftharpoons \mathrm{ICl}$ & $1.20 \cdot 10^{4}$ & & $2.90 \cdot 10^{10}$ & & & $2.40 \cdot 10^{6}$ & & \\
\hline $\mathrm{E} 88(2)$ & $\mathrm{HOI}+\mathrm{H}^{+}+\mathrm{Br}^{-} \rightleftharpoons \mathrm{IBr}$ & $5.10 \cdot 10^{6}$ & & $4.10 \cdot 10^{12}$ & & & $8.00 \cdot 10^{5}$ & & \\
\hline $\mathrm{E} 89$ (2) & $\mathrm{ICl}+\mathrm{Cl}^{-} \rightleftharpoons \mathrm{ICl}_{2}^{-}$ & $7.70 \cdot 10^{1}$ & & $4.24 \cdot 10^{9}$ & & & $5.50 \cdot 10^{7}$ & & \\
\hline $\mathrm{E} 90$ (2) & $\mathrm{IBr}+\mathrm{Br}^{-} \rightleftharpoons \mathrm{IBr}_{2}^{-}$ & $2.90 \cdot 10^{2}$ & & $4.93 \cdot 10^{6}$ & & & $1.70 \cdot 10^{5}$ & & \\
\hline E91 (2) & $\mathrm{ICl}+\mathrm{Br}^{-} \rightleftharpoons \mathrm{IClBr}^{-}$ & $1.80 \cdot 10^{4}$ & & $7.70 \cdot 10^{9}$ & & & $4.30 \cdot 10^{5}$ & & \\
\hline E92(2) & $\mathrm{IBr}+\mathrm{Cl}^{-} \rightleftharpoons \mathrm{IClBr}^{-}$ & $1.30 \cdot 10^{0}$ & & $5.00 \cdot 10^{4}$ & & & $3.80 \cdot 10^{4}$ & & \\
\hline E93 & $\mathrm{Br}_{2}+\mathrm{I}_{2} \rightleftharpoons \mathrm{IBr}+\mathrm{IBr}$ & $1.30 \cdot 10^{5}$ & & $1.30 \cdot 10^{4}$ & & 175 & $1.00 \cdot 10^{-1}$ & & 175 \\
\hline E94(2) & $\mathrm{CH}_{2} \mathrm{ICO}_{3}+\mathrm{H}_{2} \mathrm{O} \rightleftharpoons \mathrm{CH}_{2} \mathrm{IC}(\mathrm{OH})_{2} \mathrm{O}_{2}$ & $3.67 \cdot 10^{2}$ & & $1.10 \cdot 10^{7}$ & & & $3.00 \cdot 10^{4}$ & & \\
\hline
\end{tabular}


(2) already implemented in CAPRAM-HM2.1, $Q$ update of CAPRAM-HM2.1, new reactions in blue Reaction

Ref.

K

$\mathbf{E}_{\mathrm{A}} / \mathbf{R}$

Ref.

$k_{b, 298}$

$\mathbf{E}_{\mathbf{A}} / \mathbf{R}$

Ref.

E95(2)

$\mathrm{CH}_{2} \mathrm{ICOOH} \rightleftharpoons \mathrm{CH}_{2} \mathrm{ICOO}^{-}+\mathrm{H}^{+}$

$1.75 \cdot 10^{-5} \mathrm{e}^{46 / \mathrm{T}}$

$8.75 \cdot 10^{5}$

$5.00 \cdot 10^{10}$ 


\section{References}

(1) Carpenter, L. J.; Archer, S. D.; Beale, R., Ocean-atmosphere trace gas exchange. Chem. Soc. Rev. 2012, 41 (19), 6473-6506.

(2) Paulot, F.; Jacob, D. J.; Johnson, M. T.; Bell, T. G.; Baker, A. R.; Keene, W. C.; Lima, I. D.; Doney, S. C.; Stock, C. A., Global oceanic emission of ammonia: Constraints from seawater and atmospheric observations. Global Biogeochem. Cy. 2015, 29 (8), 1165-1178. (3) Lowe, D.; Topping, D.; McFiggans, G., Modelling multi-phase halogen chemistry in the remote marine boundary layer: investigation of the influence of aerosol size resolution on predicted gas- and condensed-phase chemistry. Atmos. Chem. Phys. 2009, 9 (14), 4559-4573.

(4) Wuebbles, D. J.; Hayhoe, K., Atmospheric methane and global change. Earth-Sci. Rev. 2002, 57 (3-4), 177-210.

(5) Plass-Dülmer, C.; Khedim, A.; Koppmann, R.; Johnen, F. J.; Rudolph, J.; Kuosa, H., Emissions of light nonmethane hydrocarbons from the atlantic into the atmosphere. Global Biogeochem. Cy. 1993, 7 (1), 211-228.

(6) Bonsang, B.; Polle, C.; Lambert, G., Evidence for marine production of isoprene. Geophys. Res. Lett. 1992, 19 (11), 1129-1132.

(7) Luo, G.; Yu, F., A numerical evaluation of global oceanic emissions of alpha-pinene and isoprene. Atmos. Chem. Phys. 2010, 10 (4), 2007-2015.

(8) Millet, D. B.; Jacob, D. J.; Custer, T. G.; de Gouw, J. A.; Goldstein, A. H.; Karl, T.; Singh, H. B.; Sive, B. C.; Talbot, R. W.; Warneke, C.; Williams, J., New constraints on terrestrial and oceanic sources of atmospheric methanol. Atmos. Chem. Phys. 2008, 8 (23), 6887-6905.

(9) Beale, R.; Liss, P. S.; Nightingale, P. D., First oceanic measurements of ethanol and propanol. Geophys. Res. Lett. 2010, 37 (24), L24607.

(10) Zhou, X. L.; Mopper, K., Photochemical production of low-molecular-weight carbonyl compounds in seawater and surface microlayer and their air-sea exchange. Mar. Chem. 1997, 56 (3-4), 201-213.

(11) Millet, D. B.; Guenther, A.; Siegel, D. A.; Nelson, N. B.; Singh, H. B.; de Gouw, J. A.; Warneke, C.; Williams, J.; Eerdekens, G.; Sinha, V.; Karl, T.; Flocke, F.; Apel, E.;

Riemer, D. D.; Palmer, P. I.; Barkley, M., Global atmospheric budget of acetaldehyde: 3-D model analysis and constraints from in-situ and satellite observations. Atmos. Chem. Phys. 2010, 10 (7), 3405-3425.

(12) Bräuer, P.; Tilgner, A.; Wolke, R.; Herrmann, H., Mechanism development and modelling of tropospheric multiphase halogen chemistry: The CAPRAM Halogen Module 2.0 (HM2). J. Atmos. Chem. 2013, 70 (1), 19-52.

(13) Yang, X.; Cox, R. A.; Warwick, N. J.; Pyle, J. A.; Carver, G. D.; O'Connor, F. M.; Savage, N. H., Tropospheric bromine chemistry and its impacts on ozone: A model study. $J$. Geophys. Res. 2005, 110 (D23), D23311.

(14) Butler, J. H.; King, D. B.; Lobert, J. M.; Montzka, S. A.; Yvon-Lewis, S. A.; Hall, B. D.; Warwick, N. J.; Mondeel, D. J.; Aydin, M.; Elkins, J. W., Oceanic distributions and emissions of short-lived halocarbons. Global Biogeochem. Cy. 2007, 21 (1), GB1023.

(15) Jones, C. E.; Hornsby, K. E.; Sommariva, R.; Dunk, R. M.; von Glasow, R.; McFiggans, G.; Carpenter, L. J., Quantifying the contribution of marine organic gases to atmospheric iodine. Geophys. Res. Lett. 2010, 37 (18), L18804.

(16) Lana, A.; Bell, T. G.; Simó, R.; Vallina, S. M.; Ballabrera-Poy, J.; Kettle, A. J.; Dachs, J.; Bopp, L.; Saltzman, E. S.; Stefels, J.; Johnson, J. E.; Liss, P. S., An updated climatology of surface dimethlysulfide concentrations and emission fluxes in the global ocean. Global Biogeochem. Cy. 2011, 25 (1), GB1004.

(17) Hoffmann, E. H.; Tilgner, A.; Wolke, R.; Boge, O.; Walter, A.; Herrmann, H., Oxidation of substituted aromatic hydrocarbons in the tropospheric aqueous phase: kinetic 
mechanism development and modelling. Phys. Chem. Chem. Phys. 2018, 20 (16), 1096010977.

(18) Ait-Helal, W.; Borbon, A.; Sauvage, S.; de Gouw, J. A.; Colomb, A.; Gros, V.; Freutel, F.; Crippa, M.; Afif, C.; Baltensperger, U.; Beekmann, M.; Doussin, J. F.; DurandJolibois, R.; Fronval, I.; Grand, N.; Leonardis, T.; Lopez, M.; Michoud, V.; Miet, K.; Perrier, S.; Prévôt, A. S. H.; Schneider, J.; Siour, G.; Zapf, P.; Locoge, N., Volatile and intermediate volatility organic compounds in suburban Paris: variability, origin and importance for SOA formation. Atmos. Chem. Phys. 2014, 14 (19), 10439-10464.

(19) Baker, A. K.; Beyersdorf, A. J.; Doezema, L. A.; Katzenstein, A.; Meinardi, S.; Simpson, I. J.; Blake, D. R.; Sherwood Rowland, F., Measurements of nonmethane hydrocarbons in 28 United States cities. Atmos. Environ. 2008, 42 (1), 170-182.

(20) Cahill, T. M., Ambient acrolein concentrations in coastal, remote, and urban regions in California. Environ. Sci. Technol. 2014, 48 (15), 8507-8513.

(21) Derwent, R. G.; Dernie, J. I. R.; Dollard, G. J.; Dumitrean, P.; Mitchell, R. F.; Murrells, T. P.; Telling, S. P.; Field, R. A., Twenty years of continuous high time resolution volatile organic compound monitoring in the United Kingdom from 1993 to 2012. Atmos. Environ. 2014, 99, 239-247.

(22) Fanizza, C.; Incoronato, F.; Baiguera, S.; Schiro, R.; Brocco, D., Volatile organic compound levels at one site in Rome urban air. Atmos. Pollut. Res. 2014, 5 (2), 303-314.

(23) Fuselli, S.; De Felice, M.; Morlino, R.; Turrio-Baldassarri, L., A Three Year Study on 14 VOCs at One Site in Rome: Levels, Seasonal Variations, Indoor/Outdoor Ratio and Temporal Trends. Int. J. Environ. Res. Public Health 2010, 7 (10), 3792-3803.

(24) Hoshi, J.-y.; Amano, S.; Sasaki, Y.; Korenaga, T., Investigation and estimation of emission sources of 54 volatile organic compounds in ambient air in Tokyo. Atmos. Environ. 2008, 42 (10), 2383-2393.

(25) Kuntasal, Ö. O.; Kilavuz, S. A.; Karman, D.; Wang, D.; Tuncel, G., C5-C12 volatile organic compounds at roadside, residential, and background locations in Ankara, Turkey: Temporal and spatial variations and sources. J. Air Waste Manag. Assoc. 2013, 63 (10), 11481162.

(26) Lerner, J. E.; Kohajda, T.; Aguilar, M. E.; Massolo, L. A.; Sanchez, E. Y.; Porta, A. A.; Opitz, P.; Wichmann, G.; Herbarth, O.; Mueller, A., Improvement of health risk factors after reduction of VOC concentrations in industrial and urban areas. Environ. Sci. Pollut. Res. Int. 2014, 21 (16), 9676-9688.

(27) Liu, W.; Zhang, J.; Kwon, J.; Weisel, C.; Turpin, B.; Zhang, L.; Korn, L.; Morandi, M.; Stock, T.; Colome, S., Concentrations and Source Characteristics of Airborne Carbonyl Compounds Measured Outside Urban Residences. J. Air Waste Manag. Assoc. 2012, 56 (8), 1196-1204.

(28) Masiol, M.; Agostinelli, C.; Formenton, G.; Tarabotti, E.; Pavoni, B., Thirteen years of air pollution hourly monitoring in a large city: Potential sources, trends, cycles and effects of car-free days. Sci. Total Environ. 2014, 494-495, 84-96.

(29) Okada, Y.; Nakagoshi, A.; Tsurukawa, M.; Matsumura, C.; Eiho, J.; Nakano, T., Environmental risk assessment and concentration trend of atmospheric volatile organic compounds in Hyogo Prefecture, Japan. Environ. Sci. Poll. Res. 2011, 19 (1), 201-213. (30) Paralovo, S. L.; Borillo, G. C.; Barbosa, C. G. G.; Godoi, A. F. L.; Yamamoto, C. I.; de Souza, R. A. F.; Andreoli, R. V.; Costa, P. S.; Almeida, G. P.; Manzi, A. O.; Pöhlker, C.; Yáñez-Serrano, A. M.; Kesselmeier, J.; Godoi, R. H. M., Observations of atmospheric monoaromatic hydrocarbons at urban, semi-urban and forest environments in the Amazon region. Atmos. Environ. 2016, 128, 175-184.

(31) Qin, Y.; Walk, T.; Gary, R.; Yao, X.; Elles, S., C2-C10 nonmethane hydrocarbons measured in Dallas, USA-Seasonal trends and diurnal characteristics. Atmos. Environ. 2007, $41(28), 6018-6032$. 
(32) Ramírez, N.; Cuadras, A.; Rovira, E.; Borrull, F.; Marcé, R. M., Chronic risk assessment of exposure to volatile organic compounds in the atmosphere near the largest Mediterranean industrial site. Environ. Int. 2012, 39 (1), 200-209.

(33) Rantala, P.; Järvi, L.; Taipale, R.; Laurila, T. K.; Patokoski, J.; Kajos, M. K.; Kurppa, M.; Haapanala, S.; Siivola, E.; Petäjä, T.; Ruuskanen, T. M.; Rinne, J., Anthropogenic and biogenic influence on VOC fluxes at an urban background site in Helsinki, Finland. Atmos. Chem. Phys. 2016, 16 (12), 7981-8007.

(34) Roukos, J.; Riffault, V.; Locoge, N.; Plaisance, H., VOC in an urban and industrial harbor on the French North Sea coast during two contrasted meteorological situations.

Environ. Pollut. 2009, 157 (11), 3001-3009.

(35) Saito, S.; Nagao, I.; Kanzawa, H., Characteristics of ambient C2-C11 non-methane hydrocarbons in metropolitan Nagoya, Japan. Atmos. Environ. 2009, 43 (29), 4384-4395.

(36) Sturaro, A.; Rella, R.; Parvoli, G.; Ferrara, D., Long-term phenol, cresols and BTEX monitoring in urban air. Environ. Monit. Assess. 2010, 164 (1-4), 93-100.

(37) von Schneidemesser, E.; Monks, P. S.; Plass-Duelmer, C., Global comparison of VOC and CO observations in urban areas. Atmos. Environ. 2010, 44 (39), 5053-5064.

(38) Ervens, B.; George, C.; Williams, J. E.; Buxton, G. V.; Salmon, G. A.; Bydder, M.; Wilkinson, F.; Dentener, F.; Mirabel, P.; Herrmann, H., CAPRAM 2.4 (MODAC mechanism): An extended and condensed tropospheric aqueous phase mechanism and its application. J. Geophys. Res. 2003, 108 (D14), 4426-4446.

(39) Evans, M. J.; Shallcross, D. E.; Law, K. S.; Wild, J. O. F.; Simmonds, P. G.; Spain, T. G.; Berrisford, P.; Methven, J.; Lewis, A. C.; McQuaid, J. B.; Pilling, M. J.; Bandy, B. J.; Penkett, S. A.; Pyle, J. A., Evaluation of a Lagrangian box model using field measurements from EASE (Eastern Atlantic Summer Experiment) 1996. Atmos. Environ. 2000, 34 (23), 3843-3863.

(40) Ordóñez, C.; Lamarque, J. F.; Tilmes, S.; Kinnison, D. E.; Atlas, E. L.; Blake, D. R.; Sousa Santos, G.; Brasseur, G.; Saiz-Lopez, A., Bromine and iodine chemistry in a global chemistry-climate model: description and evaluation of very short-lived oceanic sources. Atmos. Chem. Phys. 2012, 12 (3), 1423-1447.

(41) Saiz-Lopez, A.; Fernandez, R. P.; Ordóñez, C.; Kinnison, D. E.; Gómez Martín, J. C.; Lamarque, J. F.; Tilmes, S., Iodine chemistry in the troposphere and its effect on ozone. Atmos. Chem. Phys. 2014, 14 (23), 13119-13143.

(42) Hoffmann, E. H.; Tilgner, A.; Schrodner, R.; Brauer, P.; Wolke, R.; Herrmann, H., An advanced modeling study on the impacts and atmospheric implications of multiphase dimethyl sulfide chemistry. Proc. Natl. Acad. Sci. USA 2016, 113 (42), 11776-11781.

(43) Burkholder, J. B.; Sander, S. P.; Abbatt, J.; Barker, J. R.; Huie, R. E.; Kolb, C. E.; Kurylo, M. J.; Orkin, V. L.; Wilmouth, D. M.; Wine, P. H. Chemical Kinetics and Photochemical Data for Use in Atmospheric Studies, Evaluation No. 18; Jet Propulsion Laboratory: Pasadena, 2015.

(44) Ballesteros, B.; Garzon, A.; Jimenez, E.; Notario, A.; Albaladejo, J., Relative and absolute kinetic studies of 2-butanol and related alcohols with tropospheric $\mathrm{Cl}$ atoms. Phys. Chem. Chem. Phys. 2007, 9 (10), 1210-1218.

(45) Wu, H.; Mu, Y.; Zhang, X.; Jiang, G., Relative rate constants for the reactions of hydroxyl radicals and chlorine atoms with a series of aliphatic alcohols. Int. J. Chem. Kinet. 2003, 35 (2), 81-87.

(46) Lendar, M.; Aissat, A.; Cazaunau, M.; Daële, V.; Mellouki, A., Absolute and relative rate constants for the reactions of $\mathrm{OH}$ and $\mathrm{Cl}$ with pentanols. Chem. Phys. Lett. 2013, 582, 3843.

(47) Nelson, L.; Rattigan, O.; Neavyn, R.; Sidebottom, H.; Treacy, J.; Nielsen, O. J., Absolute and Relative Rate Constants for the Reactions of Hydroxyl Radicals and Chlorine 
Atoms with a Series of Aliphatic-Alcohols and Ethers at 298 K. Int. J. Chem. Kinet. 1990, 22 (11), 1111-1126.

(48) Garzon, A.; Cuevas, C. A.; Ceacero, A. A.; Notario, A.; Albaladejo, J.; FernandezGomez, M., Atmospheric reactions $\mathrm{Cl}+\mathrm{CH}_{3}-\left(\mathrm{CH}_{2}\right)_{\mathrm{n}}-\mathrm{OH}(\mathrm{n}=0-4)$ : a kinetic and theoretical study. J. Chem. Phys. 2006, 125 (10), 104305.

(49) Hurley, M. D.; Wallington, T. J.; Bjarrum, M.; Javadi, M. S.; Nielsen, O. J., Atmospheric chemistry of 3-pentanol: Kinetics, mechanisms, and products of $\mathrm{Cl}$ atom and $\mathrm{OH}$ radical initiated oxidation in the presence and absence of $\mathrm{NO}_{\mathrm{X}}$. J. Phys. Chem. A 2008, 112 (35), 8053-8060.

(50) Xue, L. K.; Saunders, S. M.; Wang, T.; Gao, R.; Wang, X. F.; Zhang, Q. Z.; Wang, W. $\mathrm{X}$., Development of a chlorine chemistry module for the Master Chemical Mechanism. Geosci. Model Dev. 2015, 8 (10), 3151-3162.

(51) Wallington, T. J.; Gierczak, C. A.; Ball, J. C.; Japar, S. M., Fourier-Transform Infrared Study of the Self Reaction of $\mathrm{C}_{2} \mathrm{H}_{5} \mathrm{O}_{2}$ Radicals in Air at 295 K. Int. J. Chem. Kinet. 1989, 21 (11), 1077-1089.

(52) Toyota, K.; Kanaya, Y.; Takahashi, M.; Akimoto, H., A box model study on photochemical interactions between VOCs and reactive halogen species in the marine boundary layer. Atmos. Chem. Phys. 2004, 4, 1961-1987.

(53) Singh, S.; Hernandez, S.; Ibarra, Y.; Hasson, A. S., Kinetics and mechanism of the reactions of n-butanal and n-pentanal with chlorine atoms. Int. J. Chem. Kinet. 2009, 41 (2), 133-141.

(54) Thevenet, R.; Mellouki, A.; Le Bras, G., Kinetics of $\mathrm{OH}$ and $\mathrm{Cl}$ reactions with a series of aldehydes. Int. J. Chem. Kinet. 2000, 32 (11), 676-685.

(55) Le Crane, J. P.; Villenave, E.; Hurley, M. D.; Wallington, T. J.; Nishida, S.; Takahashi, K.; Matsumi, Y., Atmospheric chemistry of pivalaldehyde and isobutyraldehyde: Kinetics and mechanisms of reactions with $\mathrm{Cl}$ atoms, fate of $\left(\mathrm{CH}_{3}\right)_{3} \mathrm{CC}(\mathrm{O})$ and $\left(\mathrm{CH}_{3}\right)_{2} \mathrm{CHC}(\mathrm{O})$ radicals, and self-reaction kinetics of $\left(\mathrm{CH}_{3}\right)_{3} \mathrm{CC}(\mathrm{O}) \mathrm{O}_{2}$ and $\left(\mathrm{CH}_{3}\right)_{2} \mathrm{CHC}(\mathrm{O}) \mathrm{O}_{2}$ radicals. J. Phys. Chem. A 2004, 108 (5), 795-805.

(56) Rodriguez, D.; Rodriguez, A.; Notario, A.; Aranda, A.; Diaz-de-Mera, Y.; Martinez, E., Kinetic study of the gas-phase reaction of atomic chlorine with a series of aldehydes. Atmos. Chem. Phys. 2005, 5, 3433-3440.

(57) Ullerstam, M.; Ljungström, E.; Langer, S., Reactions of acrolein, crotonaldehyde and pivalaldehyde with $\mathrm{Cl}$ atoms: structure-activity relationship and comparison with $\mathrm{OH}$ and $\mathrm{NO}_{3}$ reactions. 2001, 3 (6), 986-992.

(58) Martín, P.; Cabañas, B.; Colmenar, I.; Salgado, M. S.; Villanueva, F.; Tapia, A., Reactivity of e-butenedial with the major atmospheric oxidants. Atmos. Environ. 2013, 70, 351-360.

(59) Canosa-Mas, C. E.; Cotter, E. S. N.; Duffy, J.; Thompson, K. C.; Wayne, R. P., The reactions of atomic chlorine with acrolein, methacrolein and methyl vinyl ketone. Phys.

Chem. Chem. Phys. 2001, 3 (15), 3075-3084.

(60) Wang, W.; Ezell, M. J.; Ezell, A. A.; Soskin, G.; Finlayson-Pitts, B. J., Rate constants for the reactions of chlorine atoms with a series of unsaturated aldehydes and ketones at 298 K: structure and reactivity. Phys. Chem. Chem. Phys. 2002, 4 (10), 1824-1831.

(61) Orlando, J. J.; Tyndall, G. S.; Apel, E. C.; Riemer, D. D.; Paulson, S. E., Rate coefficients and mechanisms of the reaction of Cl-atoms with a series of unsaturated hydrocarbons under atmospheric conditions. Int. J. Chem. Kinet. 2003, 35 (8), 334-353. (62) Kaiser, E. W.; Wallington, T. J.; Hurley, M. D., Products and Mechanism of the Reaction of Chlorine Atoms with 3-Pentanone in 700-950 Torr of $\mathrm{N}_{2} / \mathrm{O}_{2}$ Diluent at 297-515 K. J. Phys. Chem. A 2010, 114 (1), 343-354. 
(63) Hasson, A. S.; Tyndall, G. S.; Orlando, J. J.; Singh, S.; Hernandez, S. Q.; Campbell, S.; Ibarra, Y., Branching Ratios for the Reaction of Selected Carbonyl-Containing Peroxy Radicals with Hydroperoxy Radicals. J. Phys. Chem. A 2012, 116 (24), 6264-6281.

(64) Sun, C. H.; Xu, B. E.; Zhang, S. W., Atmospheric Reaction of Cl plus Methacrolein: A Theoretical Study on the Mechanism, and Pressure- and Temperature-Dependent Rate Constants. J. Phys. Chem. A 2014, 118 (20), 3541-3551.

(65) Hsin, H. Y.; Elrod, M. J., Overall rate constant measurements of the reaction of hydroxy- and chloroalkylperoxy radicals derived from methacrolein and methyl vinyl ketone with nitric oxide. J. Phys. Chem. A 2007, 111 (4), 613-619.

(66) Kaiser, E. W.; Wallington, T. J.; Hurley, M. D., Products and Mechanism of the Reaction of $\mathrm{Cl}$ with Butanone in $\mathrm{N}_{2} / \mathrm{O}_{2}$ Diluent at 297-526 K. J. Phys. Chem. A 2009, 113 (11), 2424-2437.

(67) Taketani, F.; Matsumi, Y.; Wallington, T. J.; Hurley, M. D., Kinetics of the gas phase reactions of chlorine atoms with a series of ketones. Chem. Phys. Lett. 2006, 431 (4-6), $257-$ 260.

(68) Zhao, Z.; Huskey, D. T.; Nicovich, J. M.; Wine, P. H., Temperature-dependent kinetics study of the gas-phase reactions of atomic chlorine with acetone, 2-butanone, and 3pentanone. Int. J. Chem. Kinet. 2008, 40 (5), 259-267.

(69) Farrugia, L. N.; Bejan, I.; Smith, S. C.; Medeiros, D. J.; Seakins, P. W., Revised structure activity parameters derived from new rate coefficient determinations for the reactions of chlorine atoms with a series of seven ketones at $290 \mathrm{~K}$ and 1atm. Chem. Phys. Lett. 2015, 640, 87-93.

(70) Notario, A.; Mellouki, A.; Le Bras, G., Rate constants for the gas-phase reactions of chlorine atoms with a series of ketones. Int. J. Chem. Kinet. 2000, 32 (1), 62-66.

(71) Cuevas, C. A.; Notario, A.; Martinez, E.; Albaladejo, J., A kinetic study of the reaction of $\mathrm{Cl}$ with a series of linear and ramified ketones as a function of temperature. Phys. Chem. Chem. Phys. 2004, 6 (9), 2230-2236.

(72) Wang, L.; Arey, J.; Atkinson, R., Reactions of chlorine atoms with a series of aromatic hydrocarbons. Environ. Sci. Technol. 2005, 39 (14), 5302-5310.

(73) Thiault, G.; Mellouki, A.; Le Bras, G., Kinetics of gas phase reactions of $\mathrm{OH}$ and $\mathrm{Cl}$ with aromatic aldehydes. Phys. Chem. Chem. Phys. 2002, 4 (11), 2194-2199.

(74) El Dib, G.; Chakir, A.; Daële, V.; Mellouki, A., Gas-phase reaction of the $\mathrm{Cl}$ atoms with dimethylbenzaldehyde isomers. Chem. Phys. Lett. 2008, 455 (4-6), 151-155.

(75) Shi, J. C.; Bernhard, M. J., Kinetic studies of Cl-atom reactions with selected aromatic compounds using the photochemical reactor-FTIR spectroscopy technique. Int. J. Chem. Kinet. 1997, 29 (5), 349-358.

(76) Bejan, I.; Duncianu, M.; Olariu, R.; Barnes, I.; Seakins, P. W.; Wiesen, P., Kinetic study of the gas-phase reactions of chlorine atoms with 2-chlorophenol, 2-nitrophenol, and four methyl-2-nitrophenol isomers. J. Phys. Chem. A 2015, 119 (20), 4735-4745.

(77) Jenkin, M. E.; Hurley, M. A.; Wallington, T. J., Investigation of the Radical Product Channel of the $\mathrm{CH}_{3} \mathrm{OCH}_{2} \mathrm{O}_{2}+\mathrm{HO}_{2}$ Reaction in the Gas Phase. J. Phys. Chem. A 2010, 114 (1), 408-416.

(78) Wallington, T. J.; Hurley, M. D.; Ball, J. C.; Jenkin, M. E., FTIR Product Study of the Reaction of $\mathrm{CH}_{3} \mathrm{OCH}_{2} \mathrm{O}_{2}+\mathrm{HO}_{2}$. Chem. Phys. Lett. 1993, 211 (1), 41-47.

(79) Bravo, I.; Aranda, A.; Diaz-de-Mera, Y.; Moreno, E.; Tucceri, M. E.; Rodriguez, D., Kinetic, mechanistic and temperature dependence study of $\mathrm{Cl}$ reactions with $\mathrm{CH}_{3} \mathrm{OC}(\mathrm{O}) \mathrm{H}$ and $\mathrm{CH}_{3} \mathrm{CH}_{2} \mathrm{OC}(\mathrm{O}) \mathrm{H}$. Atmospheric implications. Phys. Chem. Chem. Phys. 2009, 11 (2), 384390.

(80) Wallington, T. J.; Hurley, M. D.; Maurer, T.; Barnes, I.; Becker, K. H.; Tyndall, G. S.; Orlando, J. J.; Pimentel, A. S.; Bilde, M., Atmospheric oxidation mechanism of methyl formate. J. Phys. Chem. A 2001, 105 (21), 5146-5154. 
(81) Ceacero-Vega, A. A.; Ballesteros, B.; Albaladejo, J.; Bejan, I.; Barnes, I., Temperature dependence of the gas-phase reactions of $\mathrm{Cl}$ atoms with propene and 1-butene between $285<$ $\mathrm{T}<313$ K. Chem. Phys. Lett. 2009, 484 (1-3), 10-13.

(82) Riedel, T. P.; Wolfe, G. M.; Danas, K. T.; Gilman, J. B.; Kuster, W. C.; Bon, D. M.; Vlasenko, A.; Li, S. M.; Williams, E. J.; Lerner, B. M.; Veres, P. R.; Roberts, J. M.;

Holloway, J. S.; Lefer, B.; Brown, S. S.; Thornton, J. A., An MCM modeling study of nitryl chloride $\left(\mathrm{ClNO}_{2}\right)$ impacts on oxidation, ozone production and nitrogen oxide partitioning in polluted continental outflow. Atmos. Chem. Phys. 2014, 14 (8), 3789-3800.

(83) Russell, J. J.; Seetula, J. A.; Gutman, D., Kinetics and Thermochemistry of $\mathrm{CH}_{3}, \mathrm{C}_{2} \mathrm{H}_{5}$, and $\mathrm{I}_{-} \mathrm{C}_{3} \mathrm{H}_{7}-$ Study of the Equilibrium $\mathrm{R}+\mathrm{HBr}$ Reversible R-H + Br. J. Am. Chem. Soc.

1988, 110 (10), 3092-3099.

(84) Seakins, P. W.; Pilling, M. J.; Niiranen, J. T.; Gutman, D.; Krasnoperov, L. N., Kinetics and Thermochemistry of $\mathrm{R}+\mathrm{HBr}$-Reversible-Arrow- $\mathrm{RH}+\mathrm{Br}$ Reactions Determinations of the Heat of Formation of $\mathrm{C}_{2} \mathrm{H}_{5}, \mathrm{i}-\mathrm{C}_{3} \mathrm{H}_{7}$, sec- $\mathrm{C}_{4} \mathrm{H}_{9}$, and $\mathrm{t}-\mathrm{C}_{4} \mathrm{H}_{9}$. J. Phys. Chem. - US 1992, 96 (24), 9847-9855.

(85) Imrik, K.; Dobe, S.; Berces, T., Direct kinetic parameters for the reaction of Br atoms with neopentane. Int. J. Chem. Kinet. 2001, 33 (1), 49-55.

(86) Anthony, L. M.; Roscoe, J. M., A kinetic analysis of the effect of $\mathrm{O}_{2}$ on the reactions of atomic bromine with some hydrocarbons and ethers. J. Phys. Chem. A 2004, 108 (37), 7535-7547.

(87) Ramacher, B.; Orlando, J. J.; Tyndall, G. S., Temperature-dependent rate coefficient measurements for the reaction of bromine atoms with a series of aldehydes. Int. J. Chem.

Kinet. 2000, 32 (8), 460-465.

(88) Szilagyi, I.; Imrik, K.; Dobe, S.; Berces, T., Kinetics of the reactions of bromine atoms with a series of aliphatic aldehydes at 298 K. Ber. Bunsenges. Phys. Chem. 1998, 102 (1), 7984.

(89) Farkas, E.; Kovács, G.; Szilágyi, I.; Dóbé, S.; Bérces, T.; Márta, F., Rate constant for the reaction of $\mathrm{CH}_{3} \mathrm{C}(\mathrm{O}) \mathrm{CH}_{2}$ radical with $\mathrm{HBr}$ and its thermochemical implication. Int. J. Chem. Kinet. 2006, 38 (1), 32-37.

(90) Ramacher, B.; Orlando, J. J.; Tyndall, G. S., Temperature-dependent rate coefficient measurements for the reaction of bromine atoms with trichloroethene, ethene, acetylene, and tetrachloroethene in air. Int. J. Chem. Kinet. 2001, 33 (3), 198-211.

(91) Sauer, C. G.; Barnes, I.; Becker, K. H., FT-IR kinetic and product study of the Brradical initiated oxidation of $\alpha, \beta$-unsaturated organic carbonyl compounds. Atmos. Environ. 1999, 33 (18), 2969-2979.

(92) Giri, B. R.; Roscoe, J. M., Temperature Dependence of the Rate Coefficients for the Reactions of Atomic Bromine with Toluene, Tetrahydrofuran, and Tetrahydropyran. J. Phys. Chem. A 2009, 113 (28), 8001-8010.

(93) Bierbach, A.; Barnes, I.; Becker, K. H., Rate constants of the Br-initiated gas-phase oxidation of a series of alcohols, furans and benzenes at 300 +/- 2 K. Atmos. Environ. 1999, 33 (18), 2981-2992.

(94) Plane, J. M. C.; Joseph, D. M.; Allan, B. J.; Ashworth, S. H.; Francisco, J. S., An experimental and theoretical study of the reactions OIO plus $\mathrm{NO}$ and $\mathrm{OH}$ plus OH. J. Phys.

Chem. A 2006, 110 (1), 93-100.

(95) Larin, I. K.; Nevozhai, D. V.; Spasskii, A. I.; Trofimova, E. M.; Turkin, L. E., Measurement of rate constants for the reaction of iodine monoxide with ozone. Kinet. Catal. 1999, 40 (4), 435-442.

(96) Gómez Martín, J. C.; Spietz, P.; Burrows, J. P., Kinetic and mechanistic studies of the $\mathrm{I}_{2} / \mathrm{O}_{3}$ photochemistry. J. Phys. Chem. A 2007, 111 (2), 306-320. 
(97) Kaltsoyannis, N.; Plane, J. M. C., Quantum chemical calculations on a selection of iodine-containing species (IO, OIO, $\mathrm{INO}_{3},(\mathrm{IO})_{2}, \mathrm{I}_{2} \mathrm{O}_{3}, \mathrm{I}_{2} \mathrm{O}_{4}$ and $\left.\mathrm{I}_{2} \mathrm{O}_{5}\right)$ of importance in the atmosphere. Phys. Chem. Chem. Phys. 2008, 10 (13), 1723-1733.

(98) Saunders, R. W.; Plane, J. M. C., Formation Pathways and Composition of Iodine Oxide Ultra-Fine Particles. Environ. Chem. 2005, 2 (4), 299-303.

(99) Vikis, A. C.; Macfarlane, R., Reaction of Iodine with Ozone in the Gas-Phase. J. Phys. Chem. - US 1985, 89 (5), 812-815.

(100) Gómez Martín, J. C.; Gálvez, O.; Baeza-Romero, M. T.; Ingham, T.; Plane, J. M. C.; Blitz, M. A., On the mechanism of iodine oxide particle formation. Phys. Chem. Chem. Phys. 2013, 15 (37), 15612-15622.

(101) Bedjanian, Y.; LeBras, G.; Poulet, G., Rate constants for the reactions I + OClO, I + $\mathrm{ClO}, \mathrm{Cl}+\mathrm{I}_{2}$, and $\mathrm{Cl}+\mathrm{IO}$ and heat of formation of $\mathrm{IO}$ radicals. J. Phys. Chem. - US 1996, 100 (37), 15130-15136.

(102) Bedjanian, Y.; Le Bras, G.; Poulet, G., Kinetics and mechanism of the $\mathrm{IO}+\mathrm{BrO}$ reaction. J. Phys. Chem. A 1998, 102 (51), 10501-10511.

(103) Zhang, S. L.; Strekowski, R.; Bosland, L.; Monod, A.; Zetzsch, C., Kinetic study of the reaction of $\mathrm{OH}$ with $\mathrm{CH}_{2} \mathrm{I}_{2}$. Phys. Chem. Chem. Phys. 2011, 13 (24), 11671-11677.

(104) Carpenter, L. J.; Andrews, S. J.; Lidster, R. T.; Saiz-Lopez, A.; Fernandez-Sanchez, M.; Bloss, W. J.; Ouyang, B.; Jones, R. L., A nocturnal atmospheric loss of $\mathrm{CH}_{2} \mathrm{I}_{2}$ in the remote marine boundary layer. J. Atmos. Chem. 2015, 74 (2), 145-156.

(105) Stefanopoulos, V. G.; Papadimitriou, V. C.; Lazarou, Y. G.; Papagiannakopoulos, P., Absolute rate coefficient determination and reaction mechanism investigation for the reaction of $\mathrm{Cl}$ atoms with $\mathrm{CH}_{2} \mathrm{I}_{2}$ and the oxidation mechanism of $\mathrm{CH}_{2} \mathrm{I}$ radicals. J. Phys. Chem. A 2008, 112 (7), 1526-1535.

(106) Du, S. Y.; Francisco, J. S.; Schenter, G. K.; Garrett, B. C., Interaction of ClO Radical with Liquid Water. J. Am. Chem. Soc. 2009, 131 (41), 14778-14785.

(107) Sander, R., Compilation of Henry's law constants (version 4.0) for water as solvent. Atmos. Chem. Phys. 2015, 15 (8), 4399-4981.

(108) Law, K. S.; Sturges, W. T.; Blake, D. R.; Blake, N. J.; Burkholder, J. B.; Butler, J. H.; Cox, R. A.; Haynes, P. H.; Ko, M. K. W.; Kreher, K.; Mari, C.; Pfeilsticker, K.; Plane, J. M. C.; Salawitch, R. J.; Schiller, C.; Sinnhuber, B.-M.; von Glasow, R.; Warwick, N. J.; Weubbles, D. J.; Yvon-Lewis, S. A.; Butz, A.; Considine, D. B.; Dorf, M.; Froidevaux, L.; Kovalenko, L. J.; Livesey, N. J.; Nassar, R.; Sioris, C. E.; Weisenstein, D. K. Chapter 2. Halogenated Very Short-Lived Substances; World Meteorological Organization: Geneva, Switzerland, 2007.

(109) Fuller, E. N.; Schettle.Pd; Giddings, J. C., A new method for prediction of binary gasphase diffusion coeffecients. Ind. Eng. Chem. 1966, 58 (5), 19-27.

(110) Yu, X.-Y., Critical Evaluation of Rate Constants and Equilibrium Constants of Hydrogen Peroxide Photolysis in Acidic Aqueous Solutions Containing Chloride Ions. $J$. Phys. Chem. Ref. Data 2004, 33 (3), 747-763.

(111) Jacobi, H. W.; Wicktor, F.; Herrmann, H.; Zellner, R., A laser flash photolysis kinetic study of reactions of the $\mathrm{Cl}_{2}^{-}$radical anion with oxygenated hydrocarbons in aqueous solution. Int. J. Chem. Kinet. 1999, 31 (3), 169-181.

(112) Connick, R. E., The Interaction of Hydrogen Peroxide and Hypochlorous Acid in Acidic Solutions Containing Chloride Ion. J. Am. Chem. Soc. 1947, 69 (6), 1509-1514.

(113) Mohammad, A.; Liebhafsky, H. A., The Kinetics of the Reduction of Hydrogen Peroxide by the Halides. J. Am. Chem. Soc. 1934, 56 (8), 1680-1685.

(114) Folkes, L. K.; Candeias, L. P.; Wardman, P., Kinetics and Mechanisms of Hypochlorous Acid Reactions. Arch. Biochem. Biophys. 1995, 323 (1), 120-126.

(115) Hoigne, J.; Bader, H., Kinetics of Reactions of Chlorine Dioxide (OClO) in Water. 1. Rate Constants for Inorganic and Organic-Compounds. Water Res. 1994, 28 (1), 45-55. 
(116) Domae, M.; Katsumara, Y.; Jiang, P. Y.; Nagaishi, R.; Hasegawa, C.; Ishigure, K.; Yoshida, Y., Observation of $\mathrm{ClO}_{3}$ Radical in Aqueous Chlorate Solution by Pulse-Radiolysis. J. Phys. Chem. - US 1994, 98 (1), 190-192.

(117) Haag, W. R.; Hoigne, J., Ozonation of Water Containing Chlorine or Chloramines Reaction-Products and Kinetics. Water Res. 1983, 17 (10), 1397-1402.

(118) Klaning, U. K.; Sehested, K.; Holcman, J., Standard Gibbs Energy of Formation of the Hydroxyl Radical in Aqueous-Solution - Rate Constants for the Reaction $\mathrm{ClO}_{2}^{-}+\mathrm{O}_{3}$

Revirsible $\mathrm{O}_{3}{ }^{-}+\mathrm{ClO}_{2}$. J. Phys. Chem. - US 1985, 89 (5), 760-763.

(119) Bielski, B. H. J., A Pulse-Radiolysis Study of the Reaction of Ozone with $\mathrm{Cl}_{2}{ }^{-}$

Radical-Anion in Aqueous-Solutions. Radiat. Phys. Chem. 1993, 41 (3), 527-530.

(120) Knipping, E. M.; Dabdub, D., Modeling $\mathrm{Cl}_{2}$ formation from aqueous $\mathrm{NaCl}$ particles:

Evidence for interfacial reactions and importance of $\mathrm{Cl}_{2}$ decomposition in alkaline solution. $J$. Geophys. Res. 2002, 107 (D18), 4360-4389.

(121) Huie, R. E.; Neta, P., Kinetics of One-Electron Transfer-Reactions Involving $\mathrm{ClO}_{2}$ and $\mathrm{NO}_{2}$. J. Phys. Chem. - US 1986, 90 (6), 1193-1198.

(122) Buxton, G. V.; Subhani, M. S., Radiation-Chemistry and Photochemistry of

Oxychlorine Ions .1. Radiolysis of Aqueous-Solutions of Hypochlorite and Chlorite Ions. $J$.

Chem. Soc. Farad. T. 1 1972, 68, 947-957.

(123) Dunn, R. C.; Simon, J. D., Excited-State Photoreactions of Chlorine Dioxide in Water.

J. Am. Chem. Soc. 1992, 114 (12), 4856-4860.

(124) Galajda, M.; Fodor, T.; Purgel, M.; Fábián, I., The kinetics and mechanism of the oxidation of pyruvate ion by hypochlorous acid. RSC Advances 2015, 5 (14), 10512-10520.

(125) Lehtimaa, T.; Kuitunen, S.; Tarvo, V.; Vuorinen, T., Kinetics of Aldehyde Oxidation by Chlorous Acid. Ind. Eng. Chem. Res. 2010, 49 (6), 2688-2693.

(126) Frenzel, A.; Scheer, V.; Sikorski, R.; George, C.; Behnke, W.; Zetzsch, C., Heterogeneous interconversion reactions of $\mathrm{BrNO}_{2}, \mathrm{ClNO}_{2}, \mathrm{Br}_{2}$, and $\mathrm{Cl}_{2}$. J. Phys. Chem. A 1998, 102 (8), 1329-1337.

(127) Lahoutifard, N.; Lagrange, P.; Lagrange, J., Kinetics and mechanism of nitrite oxidation by hypochlorous acid in the aqueous phase. Chemosphere 2003, 50 (10), 13491357.

(128) Buxton, G. V.; Bydder, M.; Salmon, G. A.; Williams, J. E., The reactivity of chlorine atoms in aqueous solution. Part III. The reactions of $\mathrm{Cl}^{\circ}$ with solutes. Phys. Chem. Chem. Phys. 2000, 2 (2), 237-245.

(129) Klaning, U. K.; Wolff, T., Laser Flash-Photolysis of $\mathrm{HClO}, \mathrm{ClO}^{-}, \mathrm{HBrO}$, and $\mathrm{BrO}^{-}$in Aqueous-Solution - Reactions of Cl-Atoms and Br-Atoms. Ber. Bunsen Phys. Chem. 1985, 89 (3), 243-245.

(130) Furrow, S. D.; Cervellati, R.; Amadori, G., New substrates for the oscillating BriggsRauscher reaction. J. Phys. Chem. A 2002, 106 (24), 5841-5850.

(131) Zuo, Z. H.; Katsumura, Y.; Ueda, K.; Ishigure, K., Reactions between some inorganic radicals and oxychlorides studied by pulse radiolysis and laser photolysis. J. Chem. Soc.

Faraday Trans. 1997, 93 (10), 1885-1891.

(132) Hartz, K. E. H.; Nicoson, J. S.; Wang, L.; Margerum, D. W., Kinetics and mechanisms of sulfite ion reduction of bromite and chlorite ions. Abstr. Pap. Am. Chem. S. 2002, 224, U747-U747.

(133) Gordon, G.; Tachiyashiki, S., Kinetics and Mechanism of Formation of Chlorate Ion from the Hypochlorous Acid Chlorite Ion Reaction at pH 6-10. Environ. Sci. Technol. 1991, 25 (3), 468-474.

(134) Peintler, G.; Nagypal, I.; Epstein, I. R., Systematic Design Chemical Oscillators Kinetics and Mechanism of the Reaction between Chlorite Ion and Hypochlorous Acid. $J$. Phys. Chem. - US 1990, 94 (7), 2954-2958. 
(135) Mialocq, J. C.; Gilles, L.; Hickel, B.; Lesigne, B.; Barat, F., Flash-Photolysis of Chlorine Dioxide in Aqueous-Solution. J. Phys. Chem. - US 1973, 77 (6), 742-749.

(136) Sant'Anna, R. T. P.; Santos, C. M. P.; Silva, G. P.; Ferreira, R. J. R.; Oliveira, A. P.; Cortes, C. E. S.; Faria, R. B., Kinetics and Mechanism of Chlorate-Chloride Reaction. J. Brazil. Chem. Soc. 2012, 23 (8), 1543-1550.

(137) Jayson, G. G.; Parsons, B. J.; Swallow, A. J., Some Simple, Highly Reactive, Inorganic Chlorine Derivatives in Aqueous-Solution - Their Formation Using Pulses of Radiation and Their Role in Mechanism of Fricke Dosimeter. J. Chem. Soc. Farad. T. 1 1973, (9), 1597-1607.

(138) David, F.; David, P. G., Photoredox Chemistry of Iron(III) Chloride and Iron(III) Perchlorate in Aqueous-Media - Comparative-Study. J. Phys. Chem. - US 1976, 80 (6), 579583.

(139) Nadtochenko, V. A.; Kiwi, J., Photolysis of $\mathrm{FeOH}^{2+}$ and $\mathrm{FeCl}^{2+}$ in aqueous solution. Photodissociation kinetics and quantum yields. Inorg. Chem. 1998, 37 (20), 5233-5238. (140) Shah, A. D.; Liu, Z. Q.; Salhi, E.; Hofer, T.; von Gunten, U., Peracetic acid oxidation of saline waters in the absence and presence of $\mathrm{H}_{2} \mathrm{O}_{2}$ : secondary oxidant and disinfection byproduct formation. Environ. Sci. Technol. 2015, 49 (3), 1698-1705.

(141) Prager, L.; Dowideit, P.; Langguth, H.; Schuchmann, H. P.; von Sonntag, C., Hydrolytic removal of the chlorinated products from the oxidative free-radical-induced degradation of chloroethylenes: acid chlorides and chlorinated acetic acids. J. Chem. Soc., Perkin Trans. 2 2001, (9), 1641-1647.

(142) Morozov, I.; Gligorovski, S.; Barzaghi, P.; Hoffmann, D.; Lazarou, Y. G.; Vasiliev, E.; Herrmann, H., Hydroxyl radical reactions with halogenated ethanols in aqueous solution: Kinetics and thermochemistry. Int. J. Chem. Kinet. 2008, 40 (4), 174-188.

(143) Bräuer, P. Extension and application of a tropospheric aqueous phase chemical mechanism (CAPRAM) for aerosol and cloud models Leipzig University, 2015.

(144) Candeias, L. P.; Stratford, M. R. L.; Wardman, P., Formation of Hydroxyl Radicals on Reaction of Hypochlorous Acid with Ferrocyanide, a Model IRON(II) Complex. Free Radic. Res. 1994, 20 (4), 241-249.

(145) Klaning, U. K.; Sehested, K.; Wolff, T., Ozone Formation in Laser Flash-Photolysis of Oxoacids and Oxoanions of Chlorine and Bromine. J. Chem. Soc. Faraday Trans. 1 1984, 80, 2969-2979.

(146) Zuo, Z. H.; Katsumura, Y., Formation of hydrated electron and $\mathrm{BrO}_{3}$ radical from laser photolysis of $\mathrm{BrO}_{3}{ }^{-}$aqueous solution. J. Chem. Soc. Faraday Trans. 1998, 94 (24), 3577-3580.

(147) Von Gunten, U.; Oliveras, Y., Advanced oxidation of bromide-containing waters: Bromate formation mechanisms. Environ. Sci. Technol. 1998, 32 (1), 63-70.

(148) Troy, R. C.; Margerum, D. W., Nonmetal Redox Kinetics - Hypobromite and Hypobromous Acid Reactions with Iodide and with Sulfite and the Hydrolysis of Bromosulfate. Inorg. Chem. 1991, 30 (18), 3538-3543.

(149) Beckwith, R. C.; Margerum, D. W., Kinetics of hypobromous acid disproportionation. Inorg. Chem. 1997, 36 (17), 3754-3760.

(150) Buxton, G. V.; Dainton, F. S., Radiolysis of Aqueous Solutions of Oxybromine Compounds - Spectra and Reactions Of $\mathrm{BrO}$ and $\mathrm{BrO}_{2}$. Proc. R. Soc. Lon. Ser. - A 1968, 304 (1479), 427-439.

(151) Field, R. J.; Raghavan, N. V.; Brummer, J. G., A Pulse-Radiolysis Investigation of the Reactions of $\mathrm{BrO}_{2}$. With $\mathrm{Fe}(\mathrm{Cn})_{6}{ }^{4-}, \mathrm{Mn}(\mathrm{II})$, Phenoxide Ion, and Phenol. J. Phys. Chem. - US 1982, 86 (13), 2443-2449.

(152) Szirovicza, L.; Boga, E., The kinetics of the bromate-sulfite reaction system. Int. J. Chem. Kinet. 1998, 30 (12), 869-874. 
(153) Faria, R. D.; Epstein, I. R.; Kustin, K., Kinetics of Disproportionation and $\mathrm{pK}_{\mathrm{a}}$ of Bromous Acid. J. Phys. Chem. - US 1994, 98 (4), 1363-1367.

(154) Amichai, O.; Czapski, G.; Treinin, A., Flash Photolysis of Oxybromine Anions. Israel J. Chem. 1969, 7 (3), 351-359.

(155) Nicoson, J. S.; Perrone, T. F.; Hartz, K. E. H.; Wang, L.; Margerum, D. W., Kinetics and mechanisms of the reactions of hypochlorous acid, chlorine, and chlorine monoxide with bromite ion. Inorg. Chem. 2003, 42 (19), 5818-5824.

(156) Sant'Anna, R. T.; Faria, R. B., Kinetics and Mechanism of the Chlorate-Bromide Reaction. Inorg. Chem. 2015, 54 (21), 10415-10421.

(157) Toth, Z.; Fabian, I., Kinetics and mechanism of the initial phase of the brominechlorite ion reaction in aqueous solution. Inorg. Chem. 2000, 39 (20), 4608-4614.

(158) Kumar, K.; Margerum, D. W., Kinetics and Mechanism of General-Acid-Assisted Oxidation of Bromide by Hypochlorite and Hypochlorous Acid. Inorg. Chem. 1987, 26 (16), 2706-2711.

(159) Wang, L.; Nicoson, J. S.; Hartz, K. E. H.; Francisco, J. S.; Margerum, D. W., Bromite ion catalysis of the disproportionation of chlorine dioxide with nucleophile assistance of electron-transfer reactions between $\mathrm{ClO}_{2}$ and $\mathrm{BrO}_{2}$ in basic solution. Inorg. Chem. 2002, 41 (1), 108-113.

(160) Brusa, M. A.; Colussi, A. J., A Kinetic-Study of the Reaction between Formic-Acid and HOBr. Int. J. Chem. Kinet. 1982, 14 (5), 479-485.

(161) Pelle, K.; Wittmann, M.; Lovrics, K.; Noszticzius, Z.; Liveri, M. L. T.; Lombardo, R., Mechanistic investigations of the BZ reaction with oxalic acid substrate. I. The oscillatory parameter region and rate constants measured for the reactions of $\mathrm{HOBr}, \mathrm{HBrO}_{2}$, and acidic $\mathrm{BrO}_{3}{ }^{-}$with oxalic acid. J. Phys. Chem. A 2004, 108 (25), 5377-5385.

(162) Yiin, B. S.; Margerum, D. W., Nonmetal Redox Kinetics - Reactions of Iodine and Triiodide with Sulfite and Hydrogen Sulfite and the Hydrolysis of Iodosulfate. Inorg. Chem. 1990, 29 (8), 1559-1564.

(163) Mulazzani, Q. G.; Buxton, G. V., On the kinetics and mechanism of the oxidation of $\mathrm{I}^{-}$ by $\mathrm{OH} / \mathrm{O}^{-}$in alkaline aqueous solution. Chem. Phys. Lett. 2006, 421 (1-3), 261-265.

(164) Rudich, Y.; Talukdar, R. K.; Imamura, T.; Fox, R. W.; Ravishankara, A. R., Uptake of $\mathrm{NO}_{3}$ on KI solutions: Rate coefficient for the $\mathrm{NO}_{3}+\mathrm{I}^{-}$reaction and gas-phase diffusion coefficients for $\mathrm{NO}_{3}$. Chem. Phys. Lett. 1996, 261 (4-5), 467-473.

(165) Bichsel, Y.; von Gunten, U., Determination of iodide and iodate by ion chromatography with postcolumn reaction and UV/visible detection. Anal. Chem. 1999, 71 (1), 34-38.

(166) Buxton, G. V.; Mulazzani, Q. G., On the hydrolysis of iodine in alkaline solution: A radiation chemical study. Rad. Phys. Chem. 2007, 76 (6), 932-940.

(167) Buxton, G. V.; Sellers, R. M., Radiation-Induced Redox Reactions of Iodine Species in Aqueous-Solution - Formation and Characterization of $\mathrm{I}^{\mathrm{Ii}}, \mathrm{I}^{\mathrm{IV}}, \mathrm{I}^{\mathrm{VI}}$ and $\mathrm{I}^{\mathrm{VIII}}$, the Stability of Hypoiodous Acid and the Chemistry of the Interconversion of Iodide and Iodate. J. Chem. Soc. Faraday Trans. 1 1985, 81, 449-471.

(168) De Kepper, P.; Epstein, I. R., A Mechanistic Study of Oscillations and Bistability in the Brigg Rauscher Reaction. J. Am. Chem. Soc. 1982, 104 (1), 49-55.

(169) Valkai, L.; Horvath, A. K., Compatible Mechanism for a Simultaneous Description of the Roebuck, Dushman, and Iodate-Arsenous Acid Reactions in an Acidic Medium. Inorg. Chem. 2016, 55 (4), 1595-1603.

(170) Sebők-Nagy, K.; Körtvélyesi, T., Kinetics and mechanism of the hydrolytic disproportionation of iodine. Int. J. Chem. Kinet. 2004, 36 (11), 596-602.

(171) Mezyk, S. P., Arrhenius parameter determination for the reaction of the oxide radical, hydrated electron and hydroxyl radical with iodate in aqueous solution. J. Chem. Soc.

Faraday Trans. 1996, 92 (12), 2251-2254. 
(172) Cseko, G.; Varga, D.; Horvath, A. K.; Nagypal, I., Simultaneous investigation of the Landolt and Dushman reactions. J. Phys. Chem. A 2008, 112 (26), 5954-5959.

(173) Criquet, J.; Allard, S.; Salhi, E.; Joll, C. A.; Heitz, A.; von Gunten, U., Iodate and iodo-trihalomethane formation during chlorination of iodide-containing waters: role of bromide. Environ. Sci. Technol. 2012, 46 (13), 7350-7357.

(174) Simoyi, R. H.; Masvikeni, P.; Sikosana, A., Complex Kinetics in the Bromate Iodide Reaction - a Clock Reaction-Mechanism. J. Phys. Chem. - US 1986, 90 (17), 4126-4131. (175) Chinake, C. R.; Simoyi, R. H., Kinetics and mechanism of the complex bromateiodine reaction. J. Phys. Chem. - US 1996, 100 (5), 1643-1656.

(176) Kern, D. M.; Kim, C. H., Iodine Catalysis in Chlorite-Iodide Reaction. J. Am. Chem. Soc. 1965, 87 (23), 5309-5313.

(177) Faria, R. D.; Epstein, I. R.; Kustin, K., Systematic Design of Chemical Oscillators .81. The Bromite-Iodide Clock Reaction. J. Am. Chem. Soc. 1992, 114 (18), 7164-7171.

(178) Wang, Y. L.; Nagy, J. C.; Margerum, D. W., Kinetics of Hydrolysis of Iodine Monochloride Measured by the Pulsed-Accelerated-Flow Method. J. Am. Chem. Soc. 1989, $111(20), 7838-7844$.

(179) Troy, R. C.; Kelley, M. D.; Nagy, J. C.; Margerum, D. W., Nonmetal Redox Kinetics Iodine Monobromide Reaction with Iodide-Ion and the Hydrolysis of IBr. Inorg. Chem. 1991, 30 (25), 4838-4845.

(180) Kumar, K.; Day, R. A.; Margerum, D. W., Atom-Transfer Redox Kinetics - GeneralAcid-Assisted Oxidation of Iodide by Chloramines and Hypochlorite. Inorg. Chem. 1986, 25 (24), 4344-4350.

(181) Awad, M. I.; Oritani, T.; Ohsaka, T., Kinetic studies on the oxidation of iodide by peroxyacetic acid. Inorg. Chim. Acta 2003, 344, 253-256.

(182) Fabian, I.; Gordon, G., Complex-Formation Reactions of the Chlorite Ion. Inorg. Chem. 1991, 30 (19), 3785-3787.

(183) Nicoson, J. S.; Perrone, T. F.; Hartz, K. E. H.; Wang, L.; Margerum, D. W., Kinetics and mechanism of the reaction between hypochlorous acid and bromite ion. Abstr. Pap. Am. Chem. Soc. 2002, 224, U710-U711.

(184) Lahoutifard, N.; Lagrange, P.; Lagrange, J.; Scott, S. L., Kinetics and mechanism of nitrite oxidation by $\mathrm{HOBr} / \mathrm{BrO}^{-}$in atmospheric water and comparison with oxidation by HOCl/ClO'. J. Phys. Chem. A 2002, 106 (49), 11891-11896.

(185) Wang, T. X.; Margerum, D. W., Kinetics of Reversible Chlorine Hydrolysis Temperature-Dependence and General Acid Base-Assisted Mechanisms. Inorg. Chem. 1994, 33 (6), 1050-1055.

(186) Grigorev, A. E.; Makarov, I. E.; Pikaev, A. K., Formation of $\mathrm{Cl}_{2}{ }^{-}$in the Bulk Solution during the Radiolysis of Concentrated Aqueous-Solutions of Chlorides. High Energ. Chem. 1987, 21 (2), 99-102.

(187) Zuo, Z. H.; Katsumura, Y.; Ueda, K.; Ishigure, K., Laser photolysis study on reactions of sulfate radical and nitrate radical with chlorate ion in aqueous solutions - Formation and reduction potential of $\mathrm{ClO}_{3}$ radical. J. Chem. Soc. Faraday Trans. 1997, 93 (4), 533-536. (188) Wang, L.; Margerum, D. W., Hypohalite ion catalysis of the disproportionation of chlorine dioxide. Inorg. Chem. 2002, 41 (23), 6099-6105.

(189) Behnke, W.; George, C.; Scheer, V.; Zetzsch, C., Production and decay of $\mathrm{ClNO}_{2}$ from the reaction of gaseous $\mathrm{N}_{2} \mathrm{O}_{5}$ with $\mathrm{NaCl}$ solution: Bulk and aerosol experiments. J. Geophys. Res. - Atmos. 1997, 102 (D3), 3795-3804.

(190) Beckwith, R. C.; Wang, T. X.; Margerum, D. W., Equilibrium and kinetics of bromine hydrolysis. Inorg. Chem. 1996, 35 (4), 995-1000.

(191) Bartlett, W. P.; Margerum, D. W., Temperature dependencies of the Henry's Law constant and the aqueous phase dissociation constant of bromine chloride. Environ. Sci. Technol. 1999, 33 (19), 3410-3414. 
(192) Laurence, G. S.; Ellis, K. J., Oxidation of Iodide Ion by Iron(III) Ion in AqueousSolution. J. Chem. Soc. Dalton Trans. 1972, (20), 2229-2233.

(193) Fudge, A. J.; Sykes, K. W., The Reaction between Ferric and Iodide Ions .1. Kinetics and Mechanism. J. Chem. Soc. 1952, (Jan), 119-124. 